\begin{abstract}
TITLE OF DOCUMENT: IMPROVING THE COVERAGE OF EARTH TARGETS BY MANEUVERING SATELLITE CONSTELLATIONS

MICHEL SANTOS, PH.D., 2007

DIRECTED BY:

ASSOCIATE PROFESSOR BENJAMIN SHAPIRO

AEROSPACE ENGINEERING
\end{abstract}

SATELLITE CONSTELLATIONS AROUND EARTH CAN BE USED FOR OBSERVING AND/OR COMMUNICATING WITH TARGETS ON THE SURFACE. THIS WORK ADDRESSES MANEUVERING EXISTING SATELLITE CONSTELLATIONS IN ORDER TO IMPROVE COVERAGE OF MULTIPLE TARGETS OVER A TIMESPAN OF 30 TO 120 DAYS.

A DIRECT RELATIONSHIP IS ESTABLISHED BETWEEN A SATELLITE'S ORBITAL GEOMETRY AND THE COVERAGE PROVIDED BY THAT SATELLITE. THIS IS ACCOMPLISHED BY (1) IDENTIFYING THE VIEW OF THE SATELLITE ORBIT FROM AN INERTIAL SPHERE CENTERED ON THE EARTH, AND (2) UTILIZING INFORMATION FROM ALL THE ORBITAL VIEWS 
ACROSS THE TARGET'S INERTIAL LATITUDE IN ORDER TO ARRIVE AT LOWER AND UPPER BOUNDS ON COVERAGE.

ALTERING A SATELLITE ORBIT ALSO ALTERS THE COVERAGE THAT IT PROVIDES. GAUSS' VARIATIONAL EQUATIONS ARE USED TO FIND MANEUVERING STRATEGIES THAT EFFECT MAXIMAL CHANGES IN ORBITAL GEOMETRY. THESE DISTINCT MANEUVERING STRATEGIES ARE THEN COMPILED INTO A LIST THAT WILL BE USED IN THE SUBSEQUENT OPTIMIZATION.

THE PROBLEM OF RECONFIGURING EXISTING SATELLITE CONSTELLATIONS IN ORDER TO IMPROVE COVERAGE IS PHRASED AS A MULTIOBJECTIVE OPTIMIZATION PROBLEM. IN IT, EACH SATELLITE IN THE SATELLITE CONSTELLATION CAN BE ASSIGNED ANY ONE OF THE MANEUVERING STRATEGIES AS WELL AS AN ALLOTMENT OF PROPELLANT THAT WILL BE CONSUMED DURING THE MANEUVERING. THESE BECOME THE PARAMETERS IN THE OPTIMIZATION PROBLEM.

AN ALGORITHM THAT IS WELL SUITED FOR SOLVING THIS OPTIMIZATION PROBLEM IS A MULTIOBJECTIVE EVOLUTIONARY/GENETIC ALGORITHM. SUCH AN ALGORITHM IS CAPABLE OF HANDLING, WITHOUT FURTHER TRANSFORMATIONS, THE THREE DIFFICULTIES WITH THE STATED PROBLEM: (1) CONTINUOUS AND DISCRETE OPTIMIZATION PARAMETERS (E.G. PROPELLANT ALLOTMENT, A DISTINCT MANEUVERING STRATEGY, ETC.), (2) NONLINEAR OPTIMIZATION OBJECTIVES (E.G. TOTAL COVERAGE TIME, NUMBER OF COVERAGE 
WINDOWS, ETC.), AND (3) MULTIPLE OPTIMIZATION OBJECTIVES (E.G. TOTAL COVERAGE TIME OVER TARGET 1, TOTAL COVERAGE OVER TARGET 2, ETC.). THIS ALGORITHM IS IMPLEMENTED BY ADOPTING FEATURES FROM OTHER SIMILAR ALGORITHMS.

FINALLY, A SET OF EXAMPLES IS INVESTIGATED IN ORDER TO STUDY THE EFFECTIVENESS OF THIS APPROACH. 



\title{
IMPROVING THE COVERAGE OF EARTH TARGETS BY MANEUVERING SATELLITE CONSTELLATIONS
}

\author{
By
}

\author{
Michel Santos
}
Dissertation submitted to the Faculty of the Graduate School of the University of Maryland, College Park in partial fulfillment of the requirements for the degree of
Ph.D.
2007

Advisory Committee:

Associate Professor Benjamin Shapiro, Chair

Associate Professor David Akin

Associate Professor Christopher Cadou

Associate Professor Steven Gabriel

Associate Professor Robert Sanner 
(C) Copyright by Michel Santos 2007 



\section{Dedication}

To those who serve and protect. 


\section{Acknowledgments}

I would like to thank Lilian and Casi, my parents, whose love, energy, and support throughout my entire life made this work possible.

I would like to thank Carole Mol for being a wonderful teacher and counselor throughout high school and for encouraging me to pursue studies in aerospace engineering.

I would like to thank Ben, my advisor, who encouraged me to pursue doctoral studies, who was supremely kind and patient when I messed up, and who gave me the leeway to pursue whatever line of investigation might be fruitful.

I would like to thank Scott Erwin, of the Air Force Research Laboratory, for his guidance on this topic. I would also like to jointly thank Scott and Greg Hicks for the early discussions on orbital footprints that eventually became the coverage estimation in this work.

I would like to thank my entire dissertation committee, Dr. Akin, Dr. Cadou, Dr. Gabriel, Dr. Sanner, and, again, Ben, for their fun classroom instruction and for their helpful comments which, I believe, improved this work significantly.

I would like to thank the Minta Martin Fund for Aeronautical Research and the Air Force Office of Scientific Research for sponsoring portions of my graduate research.

Finally, I would like to thank Ana Laura, my wife and best friend, for making life joyful and interesting regardless of what I do. 


\section{Table of Contents}

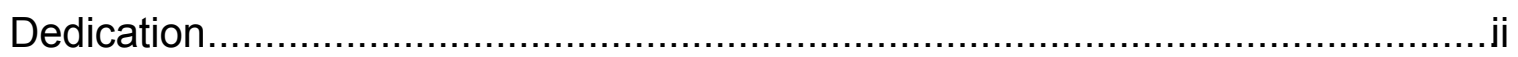

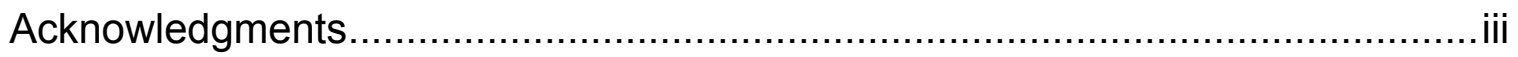

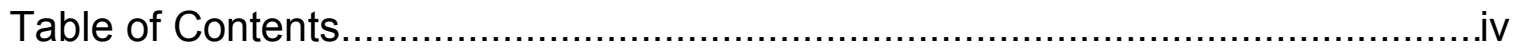

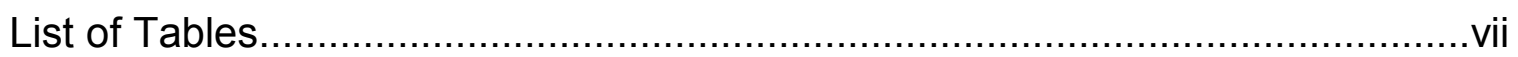

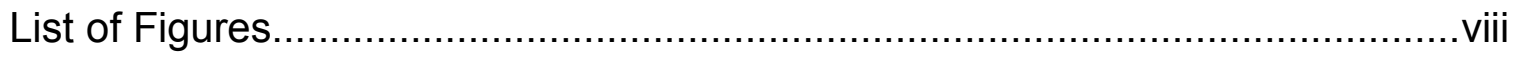

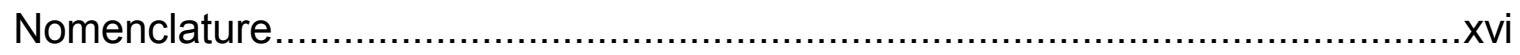

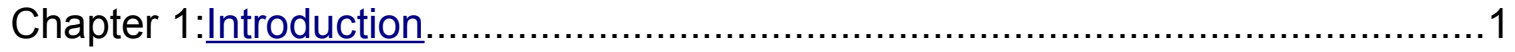

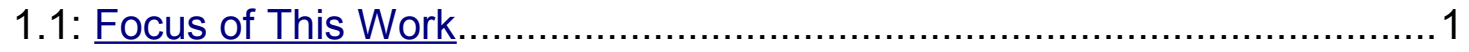

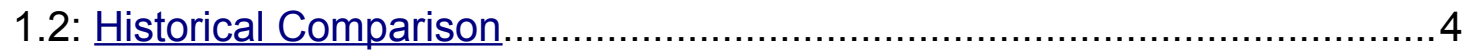

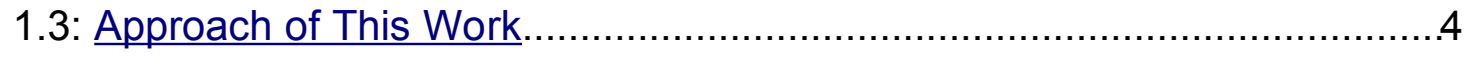

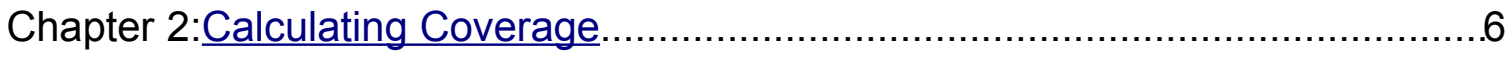

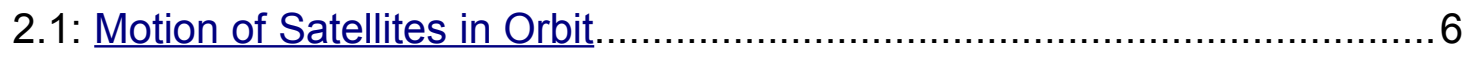

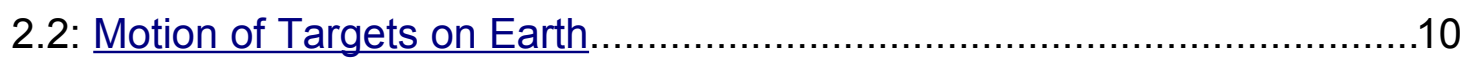

2.3: Determining Visibility between Satellites and Targets............................ 10

2.3.1: Satellite Visibility Above the Local Horizon.....................................10

2.3.2: Creation of Visibility Schedules.....................................................11

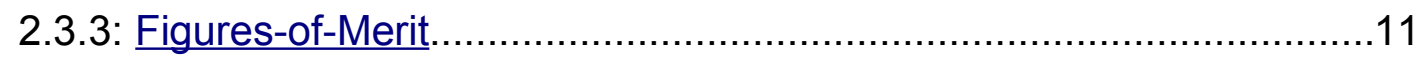

Chapter 3:Estimating Satellite Coverage ...................................................... 13

3.1: Classical Representation of an Orbit.................................................... 16

3.2: Determining the View of a Satellite Orbit from a Point on a Sphere.........19

3.2.1: Where does the LOS Cone intersect with the orbital plane? .................20

3.2.2: Intersection of the LOS cone with the orbital ellipse....................... 21

3.2.3: What are the bounds of the intersection of the LOS cone with the

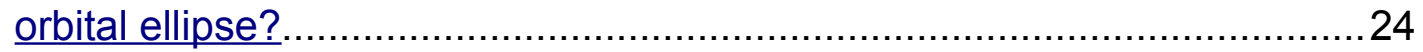

3.2.4: When can it be assured that the orbital plane will not be visible from

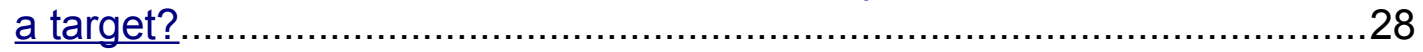

3.2.5: Is the closest intersection point closer than the ellipse's radius along

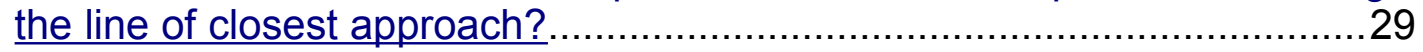

3.3: Correlating Views of a Satellite Orbit to Time along an Orbit.................. 29

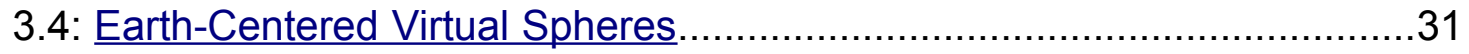

3.4.1: Virtual Sphere Aligned with Satellite Orbit.......................................31

3.4.2: Visibility Map on the Orbit-Fixed Sphere ......................................... 32 


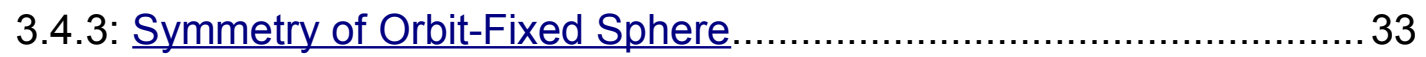

3.4.4: Earth Rotates Through a Virtual Inertial Sphere.............................. 34

3.5: Estimating Coverage from Information across a Target's Latitude............37

3.5.1: Single-Day, Single-Satellite Coverage Estimation............................37

3.5.2: Multiple-Day, Single-Satellite Coverage Estimation.......................... 43

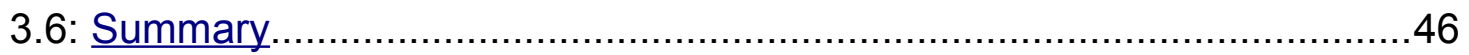

Chapter 4:Maneuvering Strategies for Maximal Changes in Orbital Geometry...47

4.1: Rate-of-Change of Keplerian Elements.......................................... 47

4.2: Maximization of the Rate-of-Change of the Right-Ascension of the

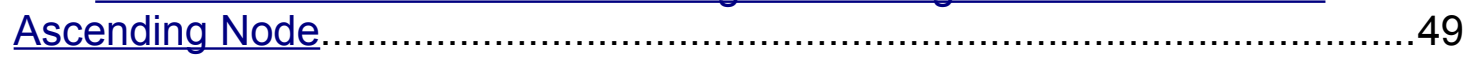

4.3: Maximization of the Rate-of-Change of Inclination...............................52

4.4: Maximization of the Rate-of-Change of the Semi-Major Axis...................56

4.5: Maximization of the Rate-of-Change of Eccentricity...............................59

4.6: Maximization of the Rate-of-Change of Argument of Periapsis............... 63

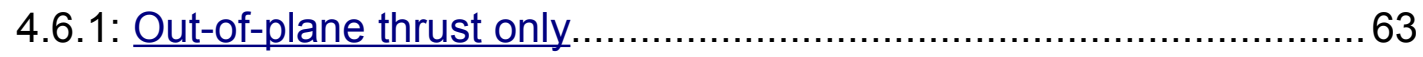

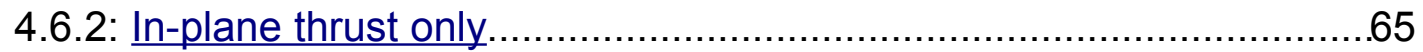

4.7: Maximization of the Rate-of-Change of the Semi-Major Axis while keeping the Eccentricity constant......

4.8: Maximization of the Rate-of-Change of Argument of Periapsis while keeping Eccentricity constant............................................................

4.9: Maximization of the Rate-of-Change of Argument of Periapsis while keeping Semi-Major Axis, Eccentricity, Inclination, and Right-Ascension of the Ascending Node Constant.......................................................................75

4.10: Maximization of the Rate-of-Change of the Semi-Major Axis and Eccentricity while keeping the Argument of Periapsis Constant......................77

4.11: Minimization of the Rate-of-Change of Any Orbital Parameter.............. 78

4.12: Maneuvering Strategy Thresholds.................................................. 79

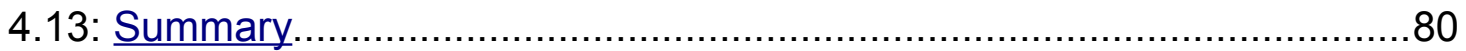

Chapter 5:Optimizing Constellation Coverage by Performing Maximal Changes

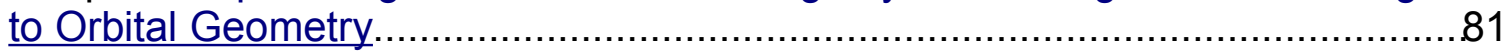

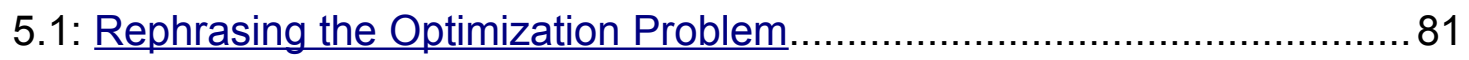

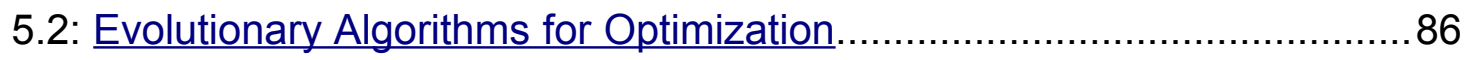

5.2.1: Single-Objective Evolutionary Algorithms....................................... 86

5.2.2: Multi-Objective Evolutionary Algorithms (MOEA)............................. 87 
5.2.3: Implementation of a Multiobjective Evolutionary Algorithm. 88

5.3: Using a Multiobjective Evolutionary Algorithm to Improve Constellation

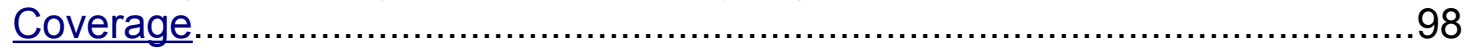

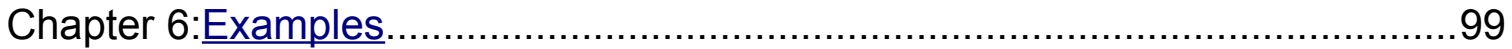

6.1: Washington, D.C. and Beijing: 30-Day, 1-Satellite.............................. 101

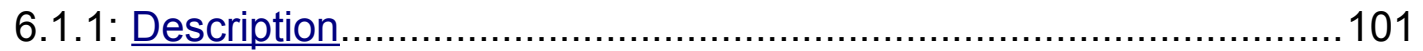

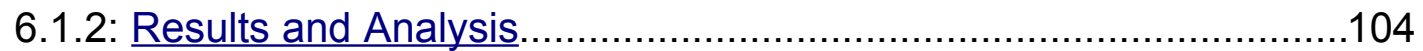

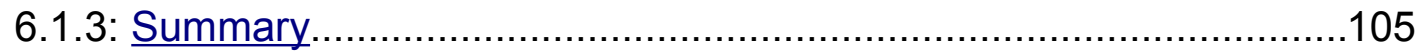

6.2: Washington, D.C. and Montevideo: 30-Day, 1-Satellite........................ 109

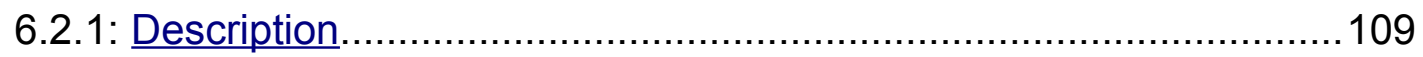

6.2.2: Results and Analysis..............................................................111

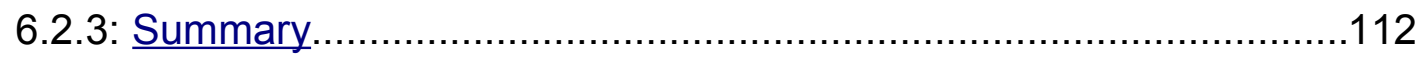

6.3: Washington, D.C. and Montevideo: 90-Day, 3-Satellites...................... 117

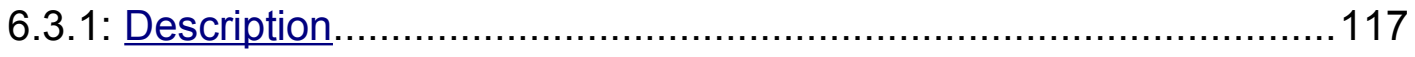

6.3.2: Results and Analysis.............................................................119

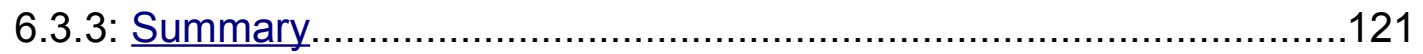

6.4: Washington, D.C. and Montevideo: 90-Day, 3-Satellites, Altitude-

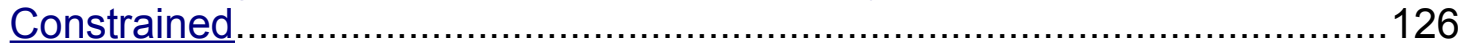

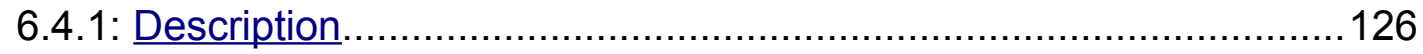

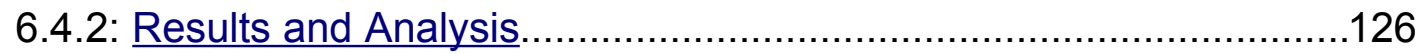

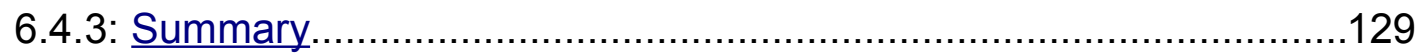

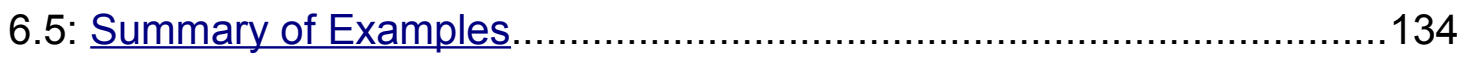

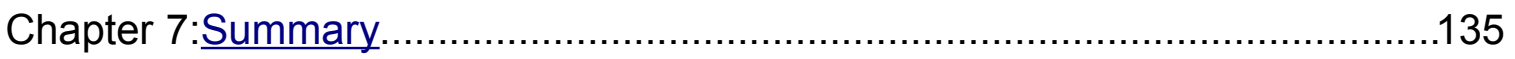

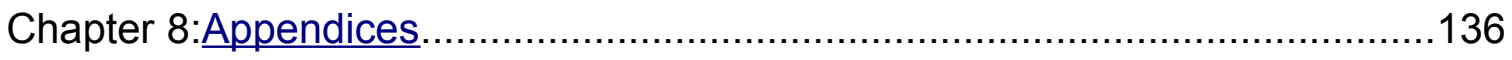

Appendix A: Summary of Operational Satellites......................................136

Appendix B: Software Modules...............................................................143

Appendix C: Supplemental Data for Chapter 6: Examples..........................147

Appendix D: Numerical Study of Single-Satellite Coverage Estimation........ 203

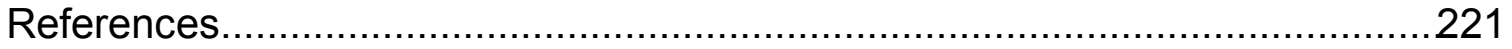




\section{List of Tables}

Table 1: Qualitative description of the coverage optimization problem................ 2

Table 2: A broad comparison of the focus of prior research to the focus of this work

Table 3: Descriptions of the classical orbital elements................................... 16

Table 4: Thresholds used by the maneuvering strategies................................79

Table 5: Qualitative description of the rephrased coverage optimization problem.. 82

Table 6: Pseudo-code for the implemented multiobjective evolution algorithm.. 88

Table 7: Pseudo-code for Selecting A Subset of a Nondominated Front............96

Table 8: Settings used for the multiobjective evolutionary algorithm for

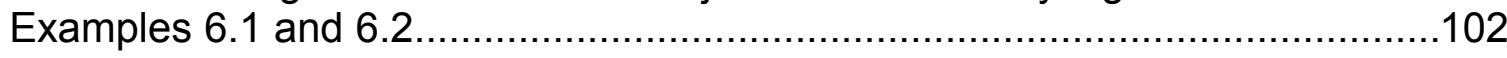

Table 9: Scenario properties for Example 6.1 …......................................103

Table 10: Scenario properties for Example 6.2 .........................................110

Table 11: Settings used for the multiobjective evolutionary algorithm for

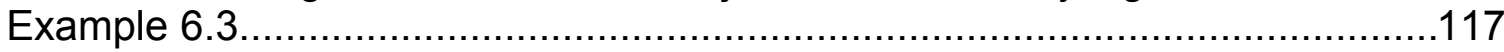

Table 12: Scenario properties for Example 6.3........................................118

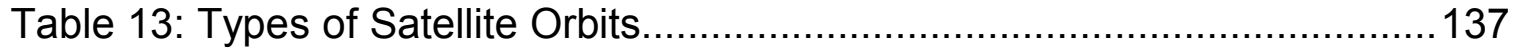

Table 14: Eccentricity by Type of Satellite Orbit......................................... 137

Table 15: Inclination by Type of Satellite Orbit............................................138

Table 16: Propellant Mass as a Percentage of Launch Mass........................... 140

Table 17: Final nondominated surface for Example 6.1............................... 147

Table 18: Final nondominated surface for Example 6.2 ................................ 153

Table 19: Final nondominated surface for Example 6.3................................ 160

Table 20: Final nondominated surface for Example 6.4 .............................. 181

Table 21: Scenario properties used for the coverage estimation study............206

Table 22: Comparison of explicit coverage versus estimated coverage across

Washington, D.C.'s longitude over 120 sidereal days.................................208

Table 23: Comparison of explicit coverage versus estimated coverage across Washington, D.C.'s longitude over the first sidereal day..............................214

Table 24: Comparison of explicit coverage versus estimated coverage across Washington, D.C.'s latitude over the first sidereal day..................................216 


\section{List of Figures}

Figure 1: Depiction of the rotating local-vertical, local-horizontal frame attached to a satellite in orbit. This reference frame is defined by three orthogonal directions: a radial direction to the satellite, the orbit normal direction, and the tangential direction which is perpendicular to the previous two directions.

Figure 2: A satellite in orbit will be able to view a location on Earth while it passes through a line-of-sight cone emanating from that location.................................11

Figure 3: Visibility schedule between targets and satellites.............................11

Figure 4: (a) Depiction of the view of Earth by an orbiting satellite. (b) Depiction of the view of a satellite orbit by an Earth target............................................... 13

Figure 5: A qualitative depiction of an LOS cone emanating from a target on the surface of the Earth. The portion of the satellite orbit that is instantaneously visible from the target is the portion that intersects the LOS cone. Since the Earth revolves around an Earth-centered inertial frame once per sidereal day (i.e. approximately 23 hours 56 minutes), both the target and its LOS cone revolve with it. Therefore, the portion of the satellite orbit that is visible from a target also changes during the sidereal day.....

Figure 6: Depiction of a Keplerian orbit and its orbital elements within an Earth-

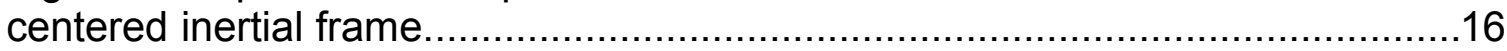

Figure 7: Depiction of a Keplerian orbit and its in-plane orbital elements.......... 16 Figure 8: Depiction of a circular, equatorial orbit within an Earth-centered inertial frame.

Figure 9: Depiction of an elliptical, equatorial orbit within an Earth-centered inertial frame.

Figure 10: Depiction of a circular, inclined orbit within an Earth-centered inertial frame

Figure 11: Depiction of an observer's LOS cone emanating from the surface of the Earth and its intersection with the orbital plane.......................................20

Figure 12: Depiction of Frame 4 and its dependence on the angular momentum vector of the satellite,

Figure 14: Depiction of an observer's LOS cone emanating from the surface of the Earth.

Figure 15: Depiction of the intersection of the planar "sides" of the LOS cone with the orbital plane.

Figure 16: The time-of-flight to travel between two points on an elliptical orbit depends on the nonlinear effect of gravity. 
Figure 17: An orbital ellipse, its associated eccentric circle, and its associated mean circle.

Figure 18: Depiction of a satellite orbit around the Earth over the course of a sidereal day. The satellite orbit and the Earth are shown relative to a Cartesian, Earth-centered inertial frame denoted by the X-Y-Z axes. Note how the Earth rotates relative to the inertial frame. Although the Earth's second-order gravity effects cause the satellite orbit to twist relative to the inertial frame, it is a weak effect on this orbit and is hardly perceptible. The dotted white lines across the surface of the Earth are the great circles on the orbit-fixed sphere representing the orbit-fixed equator and the orbit-fixed prime meridian. Since the orbit-fixed sphere is aligned with the satellite orbit, and since the satellite orbit barely moves relative to the inertial frame, the orbit-fixed equator and meridian also barely move.

Figure 19: (Left) An LOS cone is projected outwards from two locations on the orbit-fixed sphere. For both locations, the fractional view of the orbital ellipse is calculated as in Eq. (23) and appropriately color-coded. (Right) For every point on the orbit-fixed sphere, the fractional view of the orbital ellipse is calculated as in Eq. (23) and appropriately color-coded.

Figure 20: A satellite orbit and its corresponding orbit-fixed sphere. The orbitfixed sphere is symmetric across its equator and its prime meridian

Figure 21: Three cases are shown. In each case, the upper portion depicts a satellite orbit and its visibility map on the underlying inertial sphere. The lower portion depicts the surface of the inertial sphere unwrapped as a 2D surface. Areas in gray are not visible/covered by a satellite. Areas in any other color are visible/covered from a satellite. The three cases depict a satellite orbit with the same semi-major axis and eccentricity but aligned differently relative to the inertial axes. Since the semi-major axis and eccentricity are the same, all three cases share the same orbit-fixed visibility map. However, since the orbit-fixed sphere maintains its alignment with the satellite orbit, the visibility map is realigned relative to inertial space, and hence, the inertial sphere.

Figure 22: A 2D and 3D depiction of a satellite orbit and its visibility map over the course of a sidereal day. The satellite orbit and the Earth's surface are shown relative to a Cartesian, Earth-centered inertial $(E C l)$ frame denoted by the X-Y-Z axes. The Earth's surface is identifiable by the outlines of the continents (white) on the $3 \mathrm{D}$ sphere and on the 2D representation of the inertial surface. By definition, the Earth's surface rotates once relative to the $\mathrm{ECl}$ frame during the sidereal day. The visibility map of the orbit is also shown on the 3D inertial sphere and on the 2D representation. Areas in gray are not visible/covered by a satellite. Areas in any other color are visible/covered from the satellite.............. 36

Figure 23: A depiction of how a target's orbital view changes over the course of a sidereal day. By definition, the Earth completes one revolution relative to an Earth-centered inertial-frame during a sidereal day. A target on the Earth's surface will rotate with it. A target's view of a satellite orbit will change during the 
sidereal day. At some points in time (i.e. inertial longitude) the satellite's orbit will be visible to one degree or another (e.g. Sidereal Day 0.0, 0.2, 0.4, and 1.0). At other points in time, the satellite's orbit will not be visible (e.g. Sidereal Day 0.6 and 0.8$)$

Figure 24: A qualitative depiction of how the visibility between a target on the Earth and an orbiting satellite is dependent on their relative timing/phasing. A target on the surface of the Earth moves across an inertial latitude as the Earth rotates (blue) at a rate of . The satellite is in motion along its orbit (red and green) at a mean rate of. The points between A and B across the inertial latitude represent those points across the inertial latitude where line-of-sight cones (yellow) intersect the mean anomalies of MLB and MUB along the satellite orbit (green). The length of time required for the target to travel between points $A$ and $B$ is . The length of time required for the satellite to rotate between points MLB and MUB is . A target may only be seen by a satellite while the target passes between $A$ and $B$; even then, line-of-sight between the two is not attained until the satellite passes through the target's line-of-sight cone (yellow). The farther apart A and B are from each other, the greater the time that the target will be visible from the satellite orbit, thus increasing the number of viewing opportunities.

Figure 25: This variation on Fig. 24 depicts the effect that a satellite's initial mean anomaly has on the visibility between the satellite and a target on the Earth. The two plots differ because of the different initial position of the satellite. Therefore, each satellite's position (red) is shifted in time, thereby traversing the target's view of the satellite orbit (yellow) at different times.

Figure 26: Depiction of the line of apsides and the line of nodes for a classical orbital ellipse.

Figure 27: Depiction of the effect of rotation of the apsides on the satellite's orbit, and hence, its visibility map. Three points in time are shown. For each time, the upper portion depicts a satellite orbit and its visibility map on the underlying inertial sphere. The lower portion depicts the surface of the inertial sphere unwrapped as a two-dimensional surface. Areas in gray are not visible/covered by the satellite. Areas in any other color are visible/covered from the satellite. Note that this orbit is inclined to 90 degrees to nullify the effect of regression of the nodes.

Figure 28: Depiction of the effect of regression of the nodes on the satellite's orbit, and hence, its visibility map. Three points in time are shown. For each time, the upper portion depicts a satellite orbit and its visibility map on the underlying inertial sphere. The lower portion depicts the surface of the inertial sphere unwrapped as a two-dimensional surface. Areas in gray are not visible/covered by the satellite. Areas in any other color are visible/covered from the satellite. Note that this orbit is inclined at one of two critical inclinations for Earth, 63.4 degrees, in order to nullify the effect of rotation of the apsides 
Figure 29 Auxiliary function for the rate of change of right-ascension of the ascending nodes as a function of true anomaly [rad]. $(e=0.4, \omega=0) \ldots \ldots \ldots \ldots . . .51$

Figure 30 Auxiliary function for the rate of change of inclination as a function of

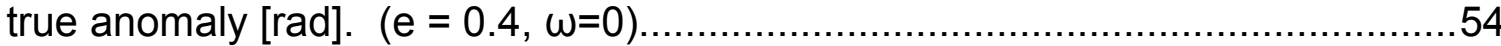

Figure 31 Auxiliary function for the rate of change of the semi-major axis as a function of true anomaly [rad]. (e=0.4).....

Figure 32 Normalized optimal thrust for maximizing the semi-major axis $(e=0.4)$. 58

Figure 33 Auxiliary function for the rate of change of eccentricity as a function of true anomaly [rad]. $(\mathrm{e}=0.4)$.

Figure 34 Normalized optimal thrust for maximizing the eccentricity $(e=0.4) \ldots 61$

Figure 35 Auxiliary function for the rate of change of the argument of periapsis

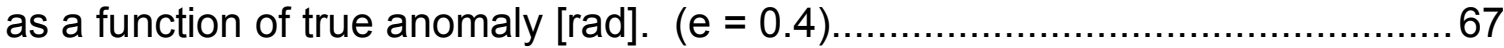

Figure 36 Normalized optimal thrust for maximizing the argument of periapsis (e $=0.4$ ).

Figure 37 The true-anomaly-derivative of the time rate-of-change of the auxiliary, argument of periapsis as a function of true anomaly [rad] $(\mathrm{e}=0.4)$. 69

Figure 38: Comparison of the optimal location for maximizing the rate-of-change of the argument of periapsis (green) versus the location where a tangential thrust has no effect on the eccentricity (blue). .73

Figure 39: Comparison of the optimal thrusting strategy to maximize the rate-ofchange of the argument of periapsis (green) versus tangential thrusting where the eccentricity is not altered (blue.).

Figure 40: Objective-space depiction of various solutions to a multiobjective maximization problem.

Figure 41: Depiction of a population of chromosomes that represent the maneuvering and propellant genes used in this work. Each chromosome contains a set of genes for each satellite in the constellation. For each satellite, there exists a maneuvering gene and a propellant gene. The maneuvering gene contains a discrete maneuvering strategy as well as a a continuous angle that is required for some strategies. The propellant gene contains a continuous variable representing the maximum amount of propellant that may be used by the satellite while executing the maneuvering strategy...

Figure 42: Depiction of the crossover genetic operator applied to two chromosomes used in this work. Given a pair of parent chromosomes, another pair of child chromosomes is created. The parent genes for a particular satellite are crossed-over in order to produce two child genes for that satellite. Some randomization in the crossover determines the level of crossover that occurs. In some cases (as shown for the red satellite), the maneuvering gene and propellant gene remain together. In some cases (as shown for the green 
satellite), the propellant swaps and pairs with the other maneuvering gene. And in some cases (as shown for the blue satellite), the propellant gene swaps along with the disambiguation angle for the maneuvering strategy. .93

Figure 43: Depiction of the mutation genetic operator applied to a chromosome used in this work. When a chromosome is mutated, at least one gene within the chromosome is mutated. During a large mutation of a maneuvering gene, the discrete maneuver will be changed as will the disambiguation angle. In contrast, during a small mutation of a maneuvering gene, only the disambiguation angle will be changed. During a large mutation of a propellant gene, the propellant allotment will be changed to some value from zero to the maximum propellant available.

Figure 44: Waves of nondominated solutions to a multiobjective maximization problem are shown

Figure 45: Graphical depiction of the Washington, D.C. and Beijing: 30-Day, 1Satellite scenario at the initial date.

Figure 46: The final generation of nondominated solutions for Example 6.1 are shown within objective space. Figure (a) shows the solutions in 3D space. Figures (b), (c), and (d) show the 3D solutions projected onto three 2D planes. As visible in the figures, the total coverage time provided by the unoptimized satellite is 0.4 hours for Washington, D.C. and 0.0 hours for Beijing....

Figure 47: Shown is the 30-day evolution of the visibility map for the original unoptimized solution (Solution 122) of Example 6.1. This solution has the satellite executing no maneuvers, and results in a total coverage of 0.4 hours for Washington, D.C. and of 0.0 hours for Beijing. Washington, D.C., Beijing, and their corresponding latitudes are marked in yellow. The continental outlines are marked in white. Areas in gray are not visible from the satellite orbit. Areas in any other color are visible/covered from the satellite orbit.

Figure 48: Shown is the 30-day evolution of the visibility map for Solution 1 of Example 6.1. This solution has the satellite executing a maneuver that maximizes coverage over Washington, D.C. (10.9 hours) and Beijing (9.7 hours). (For comparison, the total coverage time provided by the unoptimized satellite is 0.4 hours for Washington, D.C. and 0.0 hours for Beijing.) Washington, D.C., Beijing, and their corresponding latitudes are marked in yellow. The continental outlines are marked in white. Areas in gray are not visible from the satellite orbit. Areas in any other color are visible/covered from the satellite orbit. 108

Figure 49: Graphical depiction of the Washington, D.C. and Montevideo: 30-Day, 1-Satellite scenario at the initial date 109

Figure 50: The final generation of nondominated solutions for Example 6.2 are shown within objective space. Figure (a) shows the solutions in 3D space. Figures (b), (c), and (d) show the 3D solutions projected onto three 2D planes. As visible in the figures, the total coverage time provided by the unoptimized satellite is 0.4 hours for Washington, D.C. and 4.7 hours for Montevideo. 
Figure 51: Shown is the 30-day evolution of the visibility map for the original unoptimized solution (Solution 143) of Example 6.2. This solution has the satellite executing no maneuvers, and results in a total coverage of 0.4 hours for Washington, D.C. and of 4.7 hours for Montevideo. Washington, D.C., Montevideo, and their corresponding latitudes are marked in yellow. The continental outlines are marked in white. Areas in gray are not visible from the satellite orbit. Areas in any other color are visible/covered from the satellite orbit.

Figure 52: Shown is the 30-day evolution of the visibility map for Solution 1 of Example 6.2. This solution has the satellite executing a maneuver that maximizes coverage over Washington, D.C. of 11.3 hours; Montevideo's coverage is 8.76 hours. (For comparison, the total coverage time provided by the unoptimized satellite is 0.4 hours for Washington, D.C. and 4.7 hours for Montevideo.) Washington, D.C., Montevideo, and their corresponding latitudes are marked in yellow. The continental outlines are marked in white. Areas in gray are not visible from the satellite orbit. Areas in any other color are visible/covered from the satellite orbit.

Figure 53: Shown is the 30-day evolution of the visibility map for Solution 79 of Example 6.2. This solution has the satellite executing a maneuver that maximizes coverage over Montevideo of 16.3 hours; Washington, D.C.'s coverage is 4.0 hours. (For comparison, the total coverage time provided by the unoptimized satellite is 0.4 hours for Washington, D.C. and 4.7 hours for Montevideo.) Washington, D.C., Montevideo, and their corresponding latitudes are marked in yellow. The continental outlines are marked in white. Areas in gray are not visible from the satellite orbit. Areas in any other color are visible/covered from the satellite orbit.

Figure 54: Graphical depiction of the Washington, D.C. and Montevideo: 90-Day, 3-Satellite scenario at the initial date.

Figure 55: The final generation of nondominated solutions for Example 6.3 are shown within objective space. Figure (a) shows the solutions in 3D space. Figures (b), (c), and (d) show the 3D solutions projected onto three 2D planes. As visible in the figures, the total coverage time provided by the unoptimized constellation is 108.0 hours for Washington, D.C. and 36.0 hours for Montevideo. Two sets of solutions (Clusters 1 and 2) have been circled each of which reach different areas of the objective space.

Figure 56: Shown is the 90-day evolution of the visibility map for the original unoptimized solution (Solution 142) of Example 6.3. This solution has the satellite executing no maneuvers, and results in a total coverage of 108.0 hours for Washington, D.C. and 36.0 hours for Montevideo. Washington, D.C., Montevideo, and their corresponding latitudes are marked in yellow. The continental outlines are marked in white. Areas in gray are not visible from the satellite orbit. Areas in any other color are visible/covered from the satellite orbit. 
Figure 57: Shown is the 90-day evolution of the visibility map for Solution 1 of Example 6.3. This solution has the satellite executing a maneuver that maximizes coverage over Washington, D.C. of 249.8 hours; Montevideo's coverage is 93.2 hours. (For comparison, the total coverage time provided by the unoptimized satellite is 108.0 hours for Washington, D.C. and 36.0 hours for Montevideo.) Washington, D.C., Montevideo, and their corresponding latitudes are marked in yellow. The continental outlines are marked in white. Areas in gray are not visible from the satellite orbit. Areas in any other color are visible/covered from the satellite orbit.

Figure 58: Shown is the 90-day evolution of the visibility map for Solution 36 of Example 6.3. This solution has the satellite executing a maneuver that maximizes coverage over Montevideo of 117.2 hours; Washington D.C.'s coverage is 203.8 hours. (For comparison, the total coverage time provided by the unoptimized satellite is 108.0 hours for Washington, D.C. and 36.0 hours for Montevideo.) Washington, D.C., Montevideo, and their corresponding latitudes are marked in yellow. The continental outlines are marked in white. Areas in gray are not visible from the satellite orbit. Areas in any other color are visible/covered from the satellite orbit.

Figure 59: The final generation of nondominated solutions for Example 6.4 are shown within objective space. Figure (a) shows the solutions in 3D space. Figures (b), (c), and (d) show the 3D solutions projected onto three 2D planes. As visible in the figures, the total coverage time provided by the unoptimized constellation is 108.0 hours for Washington, D.C. and 36.0 hours for Montevideo. Three sets of solutions (Clusters $1 \mathrm{~b}, 3$, and 4 ) have been circled each of which reach different areas of the objective space.

Figure 60: Shown is the 90-day evolution of the visibility map for the original unoptimized solution (Solution 141) of Example 6.4. This solution has the satellite executing no maneuvers, and results in a total coverage of 108.0 hours for Washington, D.C. and 36.0 hours for Montevideo. Washington, D.C., Montevideo, and their corresponding latitudes are marked in yellow. The continental outlines are marked in white. Areas in gray are not visible from the satellite orbit. Areas in any other color are visible/covered from the satellite orbit.

Figure 61: Shown is the 90-day evolution of the visibility map for Solution 1 of Example 6.4. This solution has the satellite executing a maneuver that maximizes coverage over Washington, D.C. of 169.9 hours; Montevideo's coverage is 90.4 hours. (For comparison, the total coverage time provided by the unoptimized satellite is 108.0 hours for Washington, D.C. and 36.0 hours for Montevideo.) Washington, D.C., Montevideo, and their corresponding latitudes are marked in yellow. The continental outlines are marked in white. Areas in gray are not visible from the satellite orbit. Areas in any other color are visible/covered from the satellite orbit 
Figure 62: Shown is the 90-day evolution of the visibility map for Solution 96 of Example 6.4. This solution has the satellite executing a maneuver that maximizes coverage over Montevideo of 115.9 hours; Washington D.C.'s coverage is 130.4 hours. (For comparison, the total coverage time provided by the unoptimized satellite is 108.0 hours for Washington, D.C. and 36.0 hours for Montevideo.) Washington, D.C., Montevideo, and their corresponding latitudes are marked in yellow. The continental outlines are marked in white. Areas in gray are not visible from the satellite orbit. Areas in any other color are visible/covered from the satellite orbit.

Figure 63: Depiction of the various software modules and their relationships....... 146

Figure 64: Shown is the 120-day evolution of the visibility map for the sample orbit used in the numerical study. Washington, D.C. and its latitude are marked in yellow. The continental outlines are marked in white. Areas in gray are not visible from the satellite orbit. Areas in any other color are visible/covered from a satellite orbit.... 


\section{Nomenclature}

Unit-vector variables are denoted with an overlying "hat" (e.g. $\hat{x})$. Vectors of other magnitudes are denoted with an overlying "arrow" (e.g. $\vec{x}$ ).

a

$a_{R} \quad=$ Radial/vertical acceleration within a local-vertical, local-horizontal frame (see $\hat{e}_{R}$ )

$a_{C T} \quad=$ Cross-track acceleration within a local-vertical, local-horizontal frame pointing in the same direction as the angular momentum vector for the orbit (see $\hat{e}_{C T}$ )

$a_{\theta} \quad=$ Tangential/horizontal acceleration within a local-vertical, local-horizontal frame (see $\hat{e}_{\theta}$ )

$\mathrm{E} \quad=$ Eccentric anomaly (Section 3.3: Correlating Views of a Satellite Orbit to Time along an Orbit)

ECEF = Earth-centered Earth-fixed frame (Section 2.1:Motion of Satellites in Orbit)

$\mathrm{ECl} \quad=$ Earth-centered inertial frame (Section 2.1:Motion of Satellites in Orbit)

$\mathrm{ER} \quad=$ Earth radii (mean equatorial radius $=6378.1363 \mathrm{~km}$ from Ref. 1 )

e $\quad=$ Eccentricity (Section 3.1: Classical Representation of an Orbit)

$\hat{e}_{R} \quad=$ Radial/vertical direction within a local-vertical, local-horizontal frame (Section 4.1: Rate-of-Change of Keplerian Elements)

$\hat{e}_{C T} \quad=$ Cross-track direction within a local-vertical, local-horizontal frame; direction parallel to angular momentum vector which defines the normal to the instantaneous orbital plane (Section 4.1: Rate-of-Change of Keplerian Elements)

$\hat{e}_{\theta} \quad=$ Tangential/vertical direction within a local-vertical, local-horizontal frame; direction equal to the cross-product of $\hat{e}_{C T}$ with $\hat{e}_{R}$ (Section 4.1: Rate-ofChange of Keplerian Elements)

el $=$ Minimum elevation angle above the horizon (Section 3.2.1: Where does the LOS Cone intersect with the orbital plane?)

$F_{\max } \quad=$ Maximum applicable thrust aboard a satellite (Section 2.1:Motion of Satellites in Orbit)

$\mathrm{G} \quad=$ Universal Gravitational Constant ( $6.67259 \times 10^{-11} \frac{\mathrm{Nm}^{2}}{\mathrm{~kg}^{2}}$ from Ref. 2)

GA = Genetic Algorithm (Section 5.2: Evolutionary Algorithms for Optimization)

$i \quad=$ Inclination (Section 3.1: Classical Representation of an Orbit)

$\mathrm{kg} \quad=$ Kilogram

LOS = Line-of-sight (Section 2.3:Determining Visibility between Satellites and Targets)

LVLH = Local-vertical, local-horizontal reference frame attached to an orbiting satellite (Section 2.1:Motion of Satellites in Orbit)

M = Mean anomaly (Section 3.3: Correlating Views of a Satellite Orbit to Time along an Orbit) 


\begin{tabular}{|c|c|c|}
\hline$\dot{M}$ & & $\begin{array}{l}\text { Time-rate-of-change of the mean anomaly; mean orbital rate of motion } \\
\text { (Section 3.1: Classical Representation of an Orbit) }\end{array}$ \\
\hline$M_{\text {Earth }}$ & $=$ & Mass of the Earth $\left(5.97426 \times 10^{24} \mathrm{~kg}\right.$ used when calculating Keplerian elements) \\
\hline MOEA & $=$ & $\begin{array}{l}\text { Multi-objective evolutionary algorithm (Section 5.2: Evolutionary Algorithms for } \\
\text { Optimization) }\end{array}$ \\
\hline RAAN & $=$ & Right-ascension of the ascending node (see $\Omega$ ) \\
\hline radius $_{\min }$ & $=$ & $\begin{array}{l}\text { Minimum orbital safety radius (see Section 4.12: Maneuvering Strategy } \\
\text { Thresholds) }\end{array}$ \\
\hline SMA & $=$ & Semi-major axis (see a ) \\
\hline S & $=$ & Number of optimization objectives \\
\hline SI & $=$ & International System of Measurements \\
\hline$x$ & $=$ & X-position within a Cartesian frame (Section 2.1:Motion of Satellites in Orbit) \\
\hline$\ddot{x}$ & $=$ & X-acceleration within a Cartesian frame (Section 2.1:Motion of Satellites in Orbit) \\
\hline$y$ & $=$ & Y-position within a Cartesian frame (Section 2.1:Motion of Satellites in Orbit) \\
\hline$\ddot{y}$ & $=$ & Y- acceleration within a Cartesian frame (Section 2.1:Motion of Satellites in Orbit) \\
\hline$z$ & $=$ & Z-position within a Cartesian frame (Section 2.1:Motion of Satellites in Orbit) \\
\hline$\ddot{z}$ & $=$ & Z-acceleration within a Cartesian frame (Section 2.1:Motion of Satellites in Orbit) \\
\hline$\beta$ & $=$ & $\begin{array}{l}\text { Orientation of the LOS cone intersecting the orbital plane relative to the orbital } \\
\text { ellipse's periapsis (Section 3.2.2: Intersection of the LOS cone with the orbital } \\
\text { ellipse) }\end{array}$ \\
\hline$\Omega$ & $=$ & $\begin{array}{l}\text { Right-ascension of the ascending-node; equal to RAAN (Section 3.1: Classical } \\
\text { Representation of an Orbit) }\end{array}$ \\
\hline$\phi$ & $=$ & $\begin{array}{l}\text { Tilt of an orbital plane relative to an observer on the orbit-fixed sphere (Section } \\
\text { 3.2.1: Where does the LOS Cone intersect with the orbital plane?) }\end{array}$ \\
\hline$\omega$ & $=$ & Argument of periapsis (Section 3.1: Classical Representation of an Orbit) \\
\hline$v$ & $=$ & True anomaly (Section 3.1: Classical Representation of an Orbit) \\
\hline 2D & $=$ & Two-dimensional \\
\hline $3 \mathrm{D}$ & $=$ & Three-dimensional \\
\hline
\end{tabular}





\section{Chapter 1: Introduction}

\section{1: Focus of This Work}

Satellite constellations around Earth can be used for observing and/or communicating with targets on its surface. This work is interested in reconfiguring existing satellite constellations in order to improve coverage of multiple Earth targets over a timespan of 30 to 120 days ${ }^{\mathrm{A}}$. The primary elements that will be considered in this problem are:

1. Timespan of interest

2. Targets

3. Satellites

4. Satellite maneuvering

These four elements can be used to determine satellite coverage of targets during the timespan of interest. How this is done will be elaborated in Chapter 2: Calculating Coverage.

With maneuvering, a satellite can alter its orbit and, thereby, alter the coverage that it provides over any target. The question for this work is: how should a satellite be maneuvered in order to improve coverage? Table 1 shows this question phrased as an optimization problem.

A Although the focus of this work is on Earth, it can also be applied to other celestial bodies where:

- the first- and second-order, spherical-harmonic, gravitational forces dominate

- the rotational rate of the celestial body is approximately constant

- the periods of the satellite orbits are less than or equal to one-half of the rotational period of the celestial body 
Table 1: Qualitative description of the coverage optimization problem

\begin{tabular}{|c|l|}
\hline Optimize & $\begin{array}{l}\text { - Coverage of Target } 1 \\
\text { - Coverage of Target } 2 \\
\vdots \\
\text { - Coverage of Target } \mathrm{n}\end{array}$ \\
\hline By varying & $\begin{array}{c}\text { - Time-varying thrust-vector for Satellite } 1 \\
- \text { Time-varying thrust-vector for Satellite } 2 \\
\vdots \\
\text { Subject to }\end{array}$ \\
& $\begin{array}{l}\text { - Time-varying thrust-vector for Satellite } \mathrm{m} \\
\text { - Timespan of interest } \\
\text { - Targets and Earth equations-of-motion } \\
\text { - Initial conditions of satellite } \\
\text { - Satellite equations-of-motion } \\
\text { - Line-of-sight constraints } \\
\text { - Limits on satellite propulsion } \\
\text { - Finite thrust limits } \\
\text { - Finite propellant }\end{array}$ \\
\hline
\end{tabular}

The following shows the same optimization problem phrased in a more mathematical form.

Optimize $\quad \operatorname{Cvg}^{s}\left(t_{0}, t_{f}, \vec{S}, e l, \overline{\operatorname{Conx}}\left(t_{0}\right)\right)$ for $s=1 \cdots S$

by varying $\quad \overrightarrow{F^{r}}(t)$ for $r=1 \cdots R$

subject to $0 \mathrm{~kg} \leq m_{p}^{r}<m_{\text {sato }}^{r}$ for $r=1 \cdots R$,

$$
\begin{aligned}
& \operatorname{Sat}^{r}\left(t_{0}\right)=\left[\begin{array}{c}
x_{0}^{r} \\
y_{0}^{r} \\
z_{0}^{r} \\
\dot{x}_{0}^{r} \\
\dot{y}_{0}^{r} \\
\dot{z}_{0}^{r} \\
m_{s a t 0}^{r} \\
m_{p 0}^{r}
\end{array}\right] \text { for } r=1 \cdots R, \\
& \sqrt{x^{r}(t)^{2}+y^{r}(t)^{2}+z^{r}(t)^{2}} \geq \text { radius }_{\text {min }}>E R \text { for } r=1 \cdots R,
\end{aligned}
$$




$$
\begin{aligned}
& \frac{d}{d t} \operatorname{Sat}^{r}\left(t, \vec{F}^{r}(t)\right)=\left[\begin{array}{c}
\dot{x}^{r}\left(\vec{F}^{r}(t)\right) \\
\dot{y}^{r}\left(\vec{F}^{r}(t)\right) \\
\dot{z}^{r}\left(\vec{F}^{r}(t)\right) \\
\ddot{x}^{r}\left(\vec{F}^{r}(t)\right) \\
\ddot{y}^{r}\left(\vec{F}^{r}(t)\right) \\
\ddot{z}^{r}\left(\vec{F}^{r}(t)\right) \\
\dot{m}_{\text {sat }}^{r}\left(\vec{F}^{r}(t)\right) \\
\dot{m}_{p}^{r}\left(\vec{F}^{r}(t)\right)
\end{array}\right] \text { for } r=1 \cdots R, \text { and } \\
& \left|\vec{F}^{r}(t)\right| \leq F_{\text {max }}^{r} \text { for } r=1 \cdots R \text {, } \\
& \text { where } \quad \operatorname{Sat}^{r}(t)=\left[\begin{array}{c}
x^{r}(t) \\
y^{r}(t) \\
z^{r}(t) \\
\dot{x}^{r}(t) \\
\dot{y}^{r}(t) \\
\dot{z}^{r}(t) \\
m_{s a t}^{r}(t) \\
m_{p}^{r}(t)
\end{array}\right] \text { for } r=1 \cdots R \text {; } \\
& \overrightarrow{\operatorname{Con} x}(t)=\left[\begin{array}{c}
\operatorname{Sat}^{1}(t) \\
\operatorname{Sat}^{2}(t) \\
\vdots \\
\operatorname{Sat}^{R}(t)
\end{array}\right] \\
& t_{0} \leq t \leq t_{f} ;
\end{aligned}
$$

$S$ is the number of targets; $\mathrm{Cvg}^{\mathrm{S}}$ is a scalar function representing the coverage provided by the satellite constellation over target $s$ (see Section 2.3:

Determining Visibility between Satellites and Targets); $\vec{S}$ is a vector representation of the target locations on the surface of the Earth; $t_{0}$ is the initial time of the scenario; $t_{f}$ is the final time of the scenario; $t$ is a moment in time in the scenario; $R$ is the number of satellites in the constellation; $r$ is the index of a particular satellite; el is the minimum elevation angle above the local horizon below which any satellite is not visible from a target; $m_{p}^{r}$ is the amount of propellant mass aboard the satellite; $\vec{F}^{r}$ is a three-dimensional, vector function representing the propulsive vector for satellite $r ; F_{\max }^{r}$ is the maximum thrusting capability of satellite $r ; m_{\text {sato }}^{r}$ is the initial mass of satellite $r ; m_{\text {sat }}^{r}$ is the 
instantaneous mass of satellite $r ; \dot{m}_{\text {sat }}^{r}$ is the time rate-of-change of the mass of satellite $r$; $E R$ is the Earth's mean radius; radius $_{\text {min }}$ is the minimum orbital safety radius below which any satellite should not maneuver; $x_{0}^{r}, y_{0}^{r}$, and $z_{0}^{r}$ represent the initial Cartesian position of satellite $r ; \dot{x}_{0}^{r}, \dot{y}_{0}^{r}$, and $\dot{z}_{0}^{r}$ represent the initial Cartesian velocity of satellite $r ; \dot{x}^{r}, \dot{y}^{r}$, and $\dot{z}^{r}$ represent the instantaneous Cartesian velocity of satellite $r ; \ddot{x}^{r}, \ddot{y}^{r}$, and $\ddot{z}^{r}$ represent the instantaneous Cartesian acceleration of satellite $r$ (see Section 2.1: Motion of Satellites in Orbit); Sat ${ }^{r}$ is the instantaneous state of satellite $r$; and $\overrightarrow{\text { Conx }}$ is a vector function representing the instantaneous state of each satellite in the constellation.

Note that this optimization problem will be discussed further in Section 5.1: Rephrasing the Optimization Problem.

\section{2: Historical Comparison}

Prior research on constellation coverage can be categorized by what portion of the Earth needed to be covered and for how long. Some of the earliest work focused on time-continuous partial coverage of the middle latitudes 3,4 and of the upper latitudes 5 . The focus eventually moved on to time-continuous coverage of the entire Earth using circular orbits 6,7,8,9,10 and then to elliptical orbits 11,12,13,14. More recently, attention has moved on to time-discontinuous coverage using circular orbits ${ }^{15}$ and elliptical orbits 16,17 . An overview is provided in Ref. 18. More recently, some research has focused on finding time-discontinuous, globalcovering $19,20,21$ and region-covering 22 constellation designs using numerical optimization methods.

Such constellations are often fairly static in that, once established, these orbits either will be left alone so that their orbital properties (e.g. right-ascension of the ascending node) drift in time in a planned manner, or will be maintained constant (e.g. argument of periapsis) by use of an onboard propulsion system to counteract perturbing forces. Recent research, however, has focused on "reconfiguring" constellations for different purposes. Ref. 23 addressed maneuvering constellation satellites within the same orbital plane for the purpose of redistributing propellant. Refs. 24 and 25 investigated the staged deployment of satellites in order to increase the communication capacity of a satellite constellation. Ref. 26 investigated maneuvering constellation satellites within their initial orbital planes in order to alter the coverage provided by a multi-planar constellation. Ref. 27 sought time-continuous, global coverage by reconfiguring a known, circular constellation into another known, circular constellation.

\section{3: Approach of This Work}

This work is interested in maneuvering existing satellite constellations in order to improve coverage of multiple Earth targets over a timespan of 30 to 120 days. 
Table 2 compares and contrasts the focus of prior research with this work. It is a broad generalization made to the best of this author's knowledge.

Table 2: A broad comparison of the focus of prior research to the focus of this work

\begin{tabular}{|c|l|l|}
\hline Timespan of interest & Focus of Prior Research & \multicolumn{1}{|c|}{ Focus of This Work } \\
\hline Targets & $\begin{array}{l}\text { - Time-continuous global } \\
\text { coverage } \\
\text { - Time-continuous coverage } \\
\text { of certain latitudes } \\
\text { - Repeating ground tracks }\end{array}$ & $\begin{array}{l}\text { Coverage of multiple targets } \\
\text { on the Earth }\end{array}$ \\
\hline Satellites & $\begin{array}{l}\text { Constellation geometry } \\
\text { initially designed to ensure } \\
\text { coverage of selected } \\
\text { targets }\end{array}$ & $\begin{array}{l}\text { Initial constellation is an } \\
\text { existing set of satellites }\end{array}$ \\
\hline Satellite maneuvering & $\begin{array}{l}\text { If available, usually used to } \\
\text { maintain constellation } \\
\text { geometry }\end{array}$ & $\begin{array}{l}\text { Used to alter an existing } \\
\text { constellation's geometry } \\
\text { and, thereby, improve } \\
\text { targeted coverage }\end{array}$ \\
\hline
\end{tabular}

Chapter 2: Calculating Coverage describes how coverage is calculated for any satellite trajectory. Chapter 3: Estimating Satellite Coverage investigates what makes a satellite orbit provide good or bad coverage. It shows how orbital geometry is directly related to the coverage provided by an individual satellite. Chapter 4: Maneuvering Strategies for Maximal Changes in Orbital Geometry identifies a set of maneuvering strategies that perform maximal changes to orbital geometry. Chapter 5: Optimizing Constellation Coverage by Performing Maximal Changes to Orbital Geometry pulls these ideas together by rephrasing the original, multiobjective optimization problem described by Table 1. The transformed optimization problem is more tractable, and can be handled by the described multiobjective evolutionary algorithm. Chapter 6: Examples then applies the technique to some examples. 


\section{Chapter 2: Calculating Coverage}

This chapter addresses how to explicitly calculate the satellite coverage of an Earth target. This calculation mainly consists of three concurrent steps:

(a) simulate the motion of an orbiting satellite

(b) simulate the motion of a target on Earth's rotating surface

(c) determine the accessibility/visibility between the satellite and target

This process is repeated until the visibility has been determined over the entire timespan of interest.

\section{1: Motion of Satellites in Orbit}

Each satellite's motion around the Earth is influenced by gravitational effects and an onboard propulsion system. The center of mass of the Earth is taken as the center of an inertial frame. From Newton's Second Law of Motion, the equations of motion for the modeled satellite within this Earth-centered inertial (ECl) frame are:

$$
\begin{aligned}
\ddot{x}^{E C l} & =\ddot{x}_{g}^{E C l}+\frac{F_{x}^{E C l}}{m_{\text {sat }}}, \\
\ddot{y}^{E C l} & =\ddot{y}_{g}^{E C l}+\frac{F_{y}^{E C l}}{m_{\text {sat }}}, \\
\text { and } \quad \ddot{z}^{E C l} & =\ddot{z}_{g}^{E C l}+\frac{F_{z}^{E C l}}{m_{\text {sat }}},
\end{aligned}
$$

where $\ddot{x}, \ddot{y}$, and, $\ddot{z}$, represent the total acceleration of the satellite in the three Cartesian directions; $\ddot{x}_{g}, \ddot{y}_{g}$, and, $\ddot{z}_{g}$ are the accelerations caused by the Earth's gravity; $F_{x}, F_{y}$, and $F_{z}$ are the Cartesian components of the propulsive vector; $m_{\text {sat }}$ is the satellite's mass; and the $\mathrm{ECl}$ superscript denotes that these accelerations and forces are measured relative to an $\mathrm{ECl}$ frame.

In this work, gravity is modeled as a $3 \times 3$ spherical-harmonic gravity model 28 . The accelerations are defined within an Earth-centered Earth-fixed (ECEF) frame by the following formulas:

$$
\begin{aligned}
& \ddot{x}_{n m}^{E C E F}=\frac{G M_{\text {Earth }}}{R_{\text {Earth }}^{2}}\left\{-C_{n 0} V_{n+1,1}\right\} \text { for } m=0, \\
& \ddot{x}_{n m}^{E C E F}=\frac{G M_{\text {Earth }}}{R_{\text {Earth }}^{2}} \frac{1}{2}\left\{-C_{n m} V_{n+1, m+1}-S_{n m} W_{n+1, m+1}\right\} \\
& \left\{+\frac{G M_{\text {Earth }}}{R_{\text {Earth }}^{2}} \frac{1}{2} \frac{(n-m+2) !}{(n-m) !}\left(+C_{n m} V_{n+1, m-1}+S_{n m} W_{n+1, m-1}\right)\right\} \text { for } m>0,
\end{aligned}
$$




$$
\begin{aligned}
\ddot{y}_{n m} E C E F & =\frac{G M_{\text {Earth }}}{R_{\text {Earth }}^{2}}\left\{-C_{n 0} W_{n+1,1}\right\} \text { for } m=0, \\
\ddot{y}_{n m} E C E F & =\frac{G M_{\text {Earth }}}{R_{\text {Earth }}^{2}} \frac{1}{2}\left\{-C_{n m} W_{n+1, m+1}+S_{n m} V_{n+1, m+1}\right\} \\
& +\frac{G M_{\text {Earth }}}{R_{\text {Earth }}^{2}} \frac{1}{2} \frac{(n-m+2) !}{(n-m) !}\left(-C_{n m} W_{n+1, m-1}+S_{n m} V_{n+1, m-1}\right) \text { for } m>0, \\
\text { and } \quad \ddot{z}_{n m}{ }^{E C E F F} & =\frac{G M_{\text {Earth }}}{R_{\text {Earth }}^{2}}\left\{(n-m+1)\left(-C_{n m} V_{n+1, m}-S_{n m} W_{n+1, m}\right)\right\} \text { for } m \geq 0,
\end{aligned}
$$

where $\mathrm{G}$ is the universal gravitational constant, $M_{\text {Earth }}$ is the mass of the Earth, $n$ and $m$ respectively are the degree and order of the gravitational model ${ }^{\mathrm{B}}$, $C_{n m}$ and $S_{n m}$ are constant coefficients of the gravity model that characterize the distribution of the Earth's mass, and $V$ and $W$ are the variable coefficients of the gravitational model. These variable coefficients are calculated by using the following recursion formulas 28 :

$$
\begin{aligned}
& V_{00}=\frac{R_{\text {Earth }}}{r}, \\
& W_{00}=0 \\
& V_{n m}=\left(\frac{2 n-1}{n-m}\right) \frac{z^{E C E F} R_{\text {Earth }}}{r^{2}} V_{n-1, m}-\left(\frac{n+m-1}{n-m}\right) \frac{R_{\text {Earth }}^{2}}{r^{2}} V_{n-2, m}, \\
& W_{n m}=\left(\frac{2 n-1}{n-m}\right) \frac{z^{E C E F} R_{\text {Earth }}}{r^{2}} W_{n-1, m}-\left(\frac{n+m-1}{n-m}\right) \frac{R_{\text {Earth }}^{2}}{r^{2}} W_{n-2, m}, \\
& V_{m m}=(2 m-1)\left\{\frac{x^{E C E F} R_{\text {Earth }}}{r^{2}} V_{m-1, m-1}-\frac{y^{E C E F} R_{\text {Earth }}}{r^{2}} W_{m-1, m-1}\right\} \\
& W_{m m}=(2 m-1)\left\{\frac{x^{E C E F} \cdot R_{\text {Earth }}}{r^{2}} W_{m-1, m-1}+\frac{y^{E C E F} \cdot R_{\text {Earth }}}{r^{2}} V_{m-1, m-1}\right\}, \\
& \quad r=\sqrt{\left[x^{E C E F}\right]^{2}+\left[y^{E C E F}\right]^{2}\left[z^{E C E F}\right]^{2}},
\end{aligned}
$$

where $\ddot{x}, \ddot{y}$, and, $\ddot{z}$, represent the Cartesian position of the satellite within the ECEF frame, and $R_{\text {Earth }}$ is the mean radius of the Earth. The values used for the constant coefficients, $C_{n m}$ and $S_{n m}$, are from the Joint NASA GSFC and NIMA Geopotential Model, EGM9629.

B $n$ and $m$ are also the degree and order, respectively, of the underlying Legendre polynomials defining the spherical harmonic model. 
In order to use this gravitational model, coordinate transformations must be made between the ECEF and ECl frames. This is done by accounting for the rotation of the Earth about its axis since a particular epoch. The epoch used in this work is the J2000 frame ${ }^{30}$. The rotation of the ECEF frame relative to the $\mathrm{ECl}$ frame accounts for the Greenwich mean sidereal time, $\theta_{G S T}$, as calculated in Ref. 1, although it does not account for smaller perturbations such as nutation, precession and polar motion. The ECI and ECEF frames are related through the rotational transformation,

$$
\left\{\begin{array}{l}
x^{E C I} \\
y^{E C l} \\
z^{E C I}
\end{array}\right\}=\left[\begin{array}{ccc}
\cos \left(\theta_{G S T}\right) & \sin \left(\theta_{G S T}\right) & 0 \\
-\sin \left(\theta_{G S T}\right) & \cos \left(\theta_{G S T}\right) & 0 \\
0 & 0 & 1
\end{array}\right]\left\{\begin{array}{l}
x^{E C E F} \\
y^{E C E F} \\
z^{E C E F}
\end{array}\right\} .
$$

The onboard propulsion system is modeled as a rocket engine with a maximum thrust, $F_{\max }$. The rocket expels propellant at a relative speed, $V_{e}$, thus producing a force in the opposite direction, $\vec{F}$. The ejection of the propellant also reduces the mass of the satellite at a rate proportional to the magnitude of the force, $|\vec{F}|$, thus modeling the classical rocket equation 31 .

In this work, the direction of thrust is specified relative to a local-horizontal, local-vertical (LVLH) reference frame (see Fig. 1); the cross-track direction is defined by the cross-product of the local-vertical direction with the localhorizontal direction. (For comparison with Gauss' variational equations, which will appear later, the local-vertical direction is also referred to as the radial direction, and the local-horizontal direction is referred to as the tangential direction.) Therefore, in order to properly use the equations of motion within the inertial frame, the LVLH force vector, $\vec{F}^{L V L H}$, must be transformed to the ECI frame, $\vec{F}^{E C l}$. 


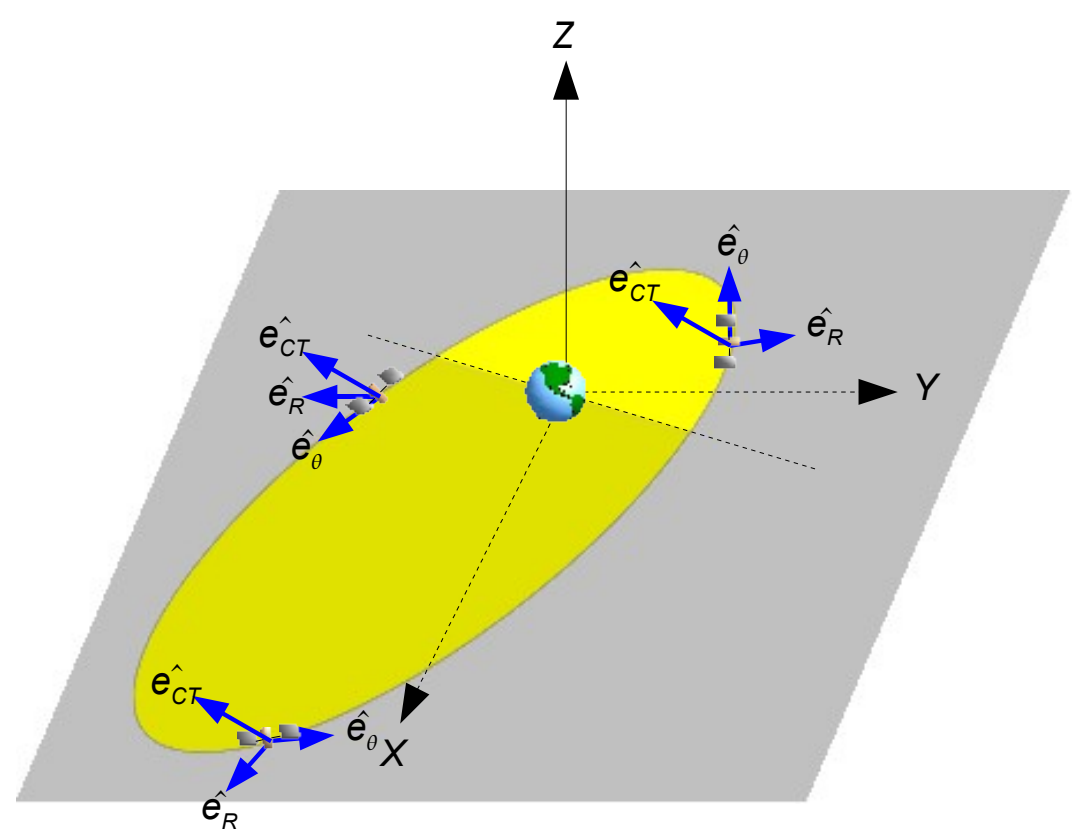

Figure 1: Depiction of the rotating local-vertical, local-horizontal frame attached to a satellite in orbit. This reference frame is defined by three orthogonal directions: a radial direction to the satellite, the orbit normal direction, and the tangential direction which is perpendicular to the previous two directions.

Once the gravitational forces and propulsions forces have been transformed into the $\mathrm{ECl}$ frame, the resulting equations of motion for any particular satellite become:

$$
\begin{aligned}
& \ddot{x}=\sum_{n, m} \ddot{x}_{n m}+\frac{F_{x}}{m_{s a t}}, \\
& \ddot{y}=\sum_{n, m} \ddot{y}_{n m}+\frac{F_{y}}{m_{\text {sat }}}, \\
& \ddot{z}=\sum_{n, m} \ddot{z}_{n m}+\frac{F_{z}}{m_{\text {sat }}}, \text { and } \\
& \dot{m}_{\text {sat }}=-\frac{|\vec{F}|}{V_{e}},
\end{aligned}
$$

subject to $|\vec{F}|=\sqrt{\left(F_{x}^{2}+F_{y}^{2}+F_{z}^{2}\right)} \leq F_{\text {max }}$,

where $|\vec{F}|$ is the magnitude of the propulsive vector; $F_{x}, F_{y}$, and $F_{z}$ are Cartesian components of the propulsive vector in the ECl frame; $m_{\text {sat }}$ is the vehicle's mass; $\dot{m}_{\text {sat }}$ is the rate of change of the vehicle's mass; $V_{e}$ is the exit velocity of the propellant; and $F_{\max }$ is the maximum thrusting capability.

In this work, the numerical integration of these equations-of-motion is carried out using the Runge-Kutta-Fehlberg $4^{\text {th }}-5^{\text {th }}$ order algorithm ${ }^{32}$ which uses an 
adaptive step-size to keep the error at each time-step below a specified level. The level specified in this work is $10^{-6}$. The local error is calculated as the square root of the sum of the square of the position error and the square of the velocity error. Since the position error has dimensions of length, and since the velocity error has dimensions of length over time, they must be nondimensionalized in such a way that an error in one is subjectively equal to an error in the other. An energy comparison was made for a low-Earth orbiting satellite. Specifically, the change in potential energy as a function of a radialposition error was compared with the change in kinetic energy as a function of a speed error. It was decided that position errors would be measured in meters and that speed errors would be measured in 0.1 meters-per-second.

\section{2: Motion of Targets on Earth}

The targets are considered fixed on the surface of the Earth. The surface is modeled as an oblate spheroid 1 with an eccentricity of 0.0033536431 . The amount of time required by Earth to rotate 360 degrees in the $\mathrm{ECl}$ frame is equal to one sidereal day, which is equivalent to $0.99726963 \mathrm{SI}^{\text {days }}{ }^{33}$. Therefore, Earth's average rate of rotation is set equal to 360 degrees per sidereal day.

\section{3: Determining Visibility between Satellites and Targets}

\subsection{1: Satellite Visibility Above the Local Horizon}

At any instant in time, a line-of-sight (LOS) vector can be calculated between each target and each satellite. In order for the line-of-sight to be useful for communications/observations, the vector should pass high enough above the local horizon to avoid obscuration by local surroundings, such as buildings and trees, and to minimize atmospheric refraction. Therefore, a minimum elevation angle above the local horizon is necessary for a useful line-of-sight. When this minimum elevation angle is uniform across all local azimuth directions, the lineof-sight emanates like a cone from the target (see Fig. 2). A satellite is only accessible/visible from a target while the satellite passes through this cone.

In this work, the shape of the Earth's surface is modeled as an oblate spheroid, rather than merely a sphere, in order to address the concern ${ }^{34}$ of underreporting coverage for targets near the Earth's poles. Therefore, when calculating the elevation of the line-of-sight vector relative to the horizon, this work calculates the local horizon as a function of the target's geodetic latitude, which is the latitude on the oblate spheroid representing the Earth 1 , as opposed to its geocentric latitude, which is the latitude on the idealized sphere representing the Earth. 


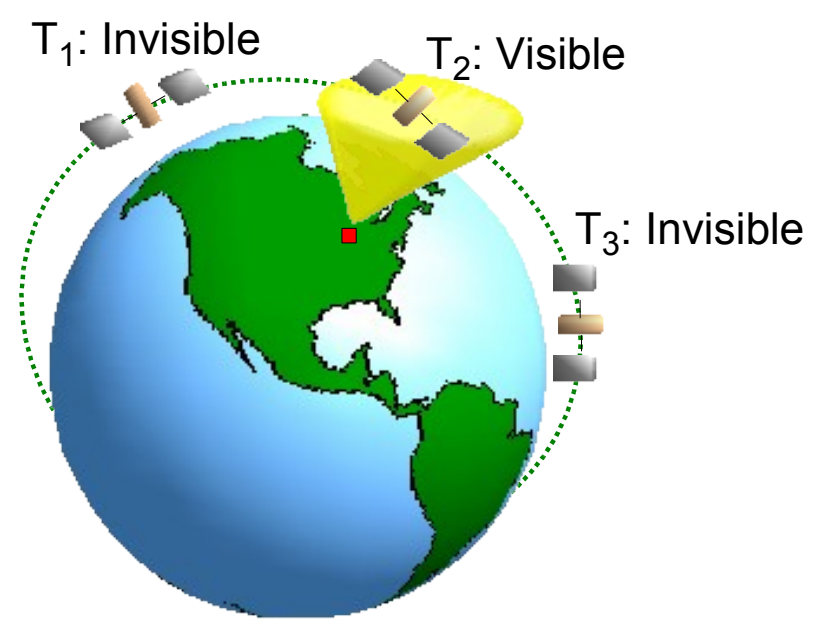

Figure 2: A satellite in orbit will be able to view a location on Earth while it passes through a line-ofsight cone emanating from that location.

\subsection{2: Creation of Visibility Schedules}

The visibility between each target and each satellite is calculated at sixsecond intervals within the timespan of a given scenario. Times during which a target/satellite is visible are classified as "windows" of coverage. Times between windows of visibility are classified as "gaps" in coverage.

Figure 3 depicts a generalized visibility schedule for two targets and two satellites. Note that Satellite 1 provides only one coverage window, and that it is of Target 1 . In contrast, Satellite 2 can view Targets 1 and 2 over multiple windows. Note how the overlap of some of Satellite 2's windows allow it to observe more than one target at a time.

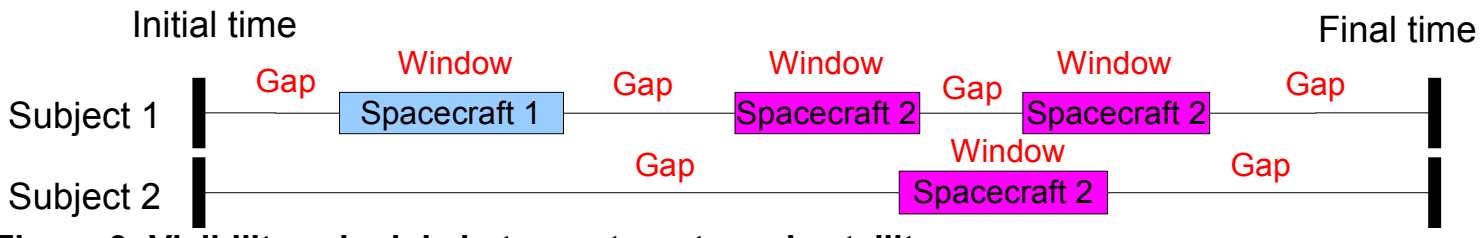

Figure 3: Visibility schedule between targets and satellites

\subsection{3: Figures-of-Merit}

The quality of coverage provided by any constellation for a set of targets is assessed by figures-of-merit 35 (FOM). Figures-of-merit are functions of the visibility schedule. One figure-of-merit measures the total amount of time during which a target is accessible/visible by at least one satellite. Another figure-ofmerit measures the average time-gap in coverage during which a target is not accessible/visible by any satellite. Either one of these figures-of-merit may be discontinuous as a window of coverage pops in and out of observability by the 
constellation (see Fig. 3). (Recall that this work is interested in optimizing multiple figures-of-merit.) Further consideration must be given for coverage windows that are too brief for practical purposes. This work will not consider coverage windows less than a user-specified duration.

Williams et al.20 observed that whereas it is often desirable to minimize the maximum time-gap, doing so often conflicts with minimizing the average timegap. Furthermore, a purely numerical assessment of coverage can sometimes yield unexpected results. Wertz et al. 35 recount a mission analysis where, in order to minimize the average time-gap of a constellation, the plan was to add $50 \%$ more satellites. Surprisingly, their simulated results indicated that the average time-gap increased by $50 \%$. A careful comparison of the visibility schedules identified the problem. "We had improved the coverage by adding satellites and had actually filled in quite a few of the smaller gaps. What was left was a small number of larger gaps such that the average gap duration was longer, even though the total of all of the gaps was significantly reduced." A careful analysis of any optimization's results is always necessary. 


\section{Chapter 3: Estimating Satellite Coverage}

Prior research on constellation coverage designed for optimum visibility in one of two ways. The first way was to geometrically arrange satellite constellations such that they provide time-continuous coverage of targets on the surface of the Earth (e.g. multiple geosynchronous satellites or Walker constellations $\left.^{6}\right)$. When time-continuous coverage is not possible or desirable, a few analytical approximations are available which estimate how much of the Earth can be seen by a satellite in circular, low- to medium- orbit. When further taking into account the period of the orbit, an estimate can be made about how long a satellite can observe a particular target. Although Wertz et al. summarize these analytical approximations, they insert an important caveat at the beginning of their summary: "All of the formulas here take into account the spherical surface of the Earth, but do not account for oblateness, orbit eccentricity, or the rotation of the Earth underneath the orbit. These effects, in addition to those of coverage by multiple satellites, are ordinarily accounted for in numerical simulations," 35 and are described in Section 2.3: Determining Visibility between Satellites and Targets.

The analytical formulas described by Wertz et al. rely on estimating how much of the Earth can be seen by a satellite in orbit. The analysis assumes, like many other works, an orientation of the satellite's sensors (usually assumed to be nadir-pointing $3,6,10,36$ ), and then projects a line-of-sight cone that impinges on the surface of the Earth (approximately represented by a spherical surface $3,6,10,36,37$ ) to arrive at a coverage footprint (usually circular6,10,36).

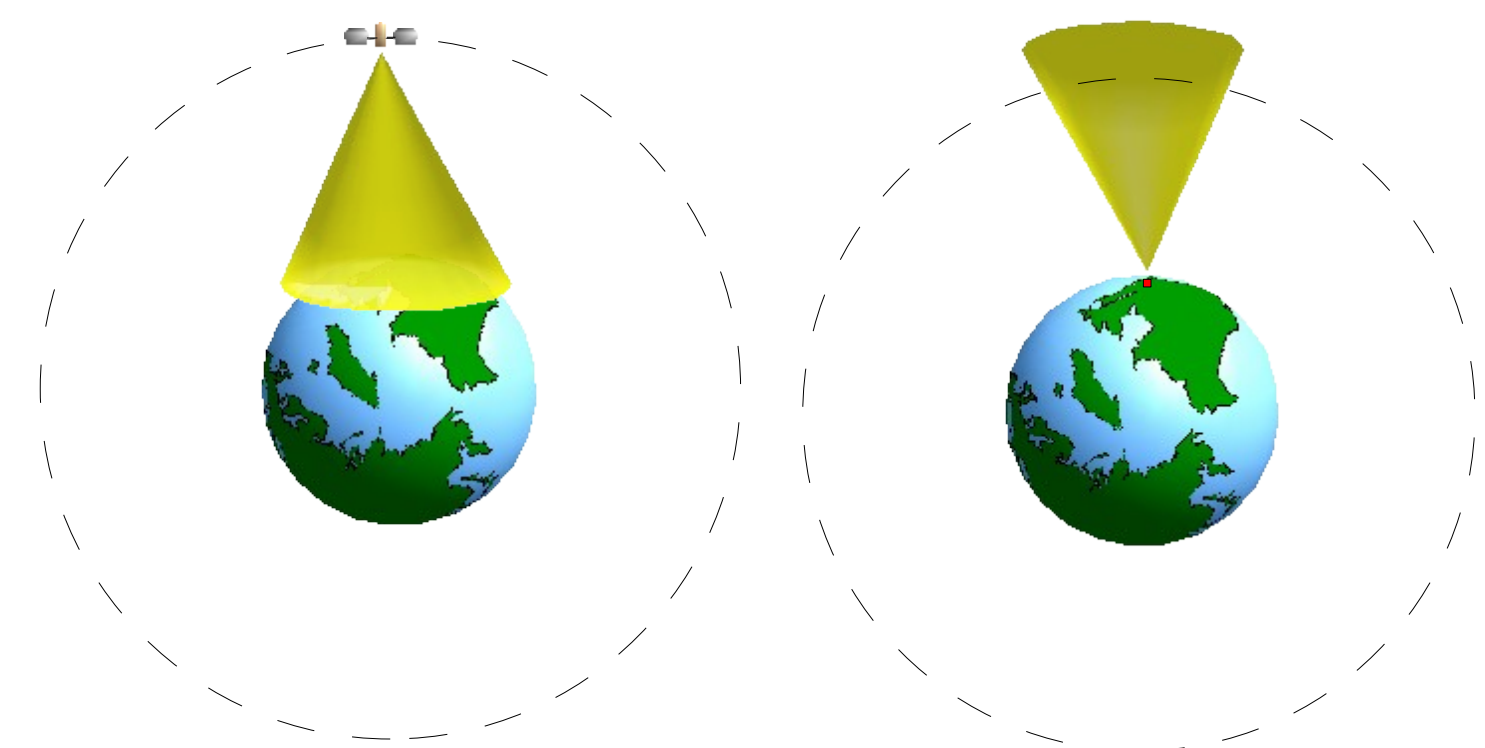

Figure 4: (a) Depiction of the view of Earth by an orbiting satellite. (b) Depiction of the view of a satellite orbit by an Earth target.

A problem with this approach is that it does not account for how visible a satellite is from the perspective of a target on the Earth ${ }^{36}$. As an extreme 
example, consider a satellite in orbit with a field-of-view large enough to encompass the entire Earth (See Fig. 4a). Although one-half of the Earth is visible from the satellite, consider the line-of-sight from the perspective of a target on the extreme edges of the visible portion: the satellite is very low on the horizon. The line-of-sight will have to contend with obstructions caused by trees or buildings, as well as a longer path through the atmosphere which tends to disturb and refract the passage of light.

The analysis in this chapter proceeds from the perspective of a target on the surface of the Earth (see Fig.4b). Given a minimum elevation angle above which communication/observation of a satellite can be assured, project a zenithpointing, line-of-sight (LOS) cone outwards and identify which portion of a satellite orbit is intersected ${ }^{\mathrm{C}}$. In effect, this addresses the coverage problem in the other direction by asking, "What portion of a satellite orbit can be seen from the target?" It is assumed that if a target on the surface of the Earth can view a satellite then the satellite can also view the target.

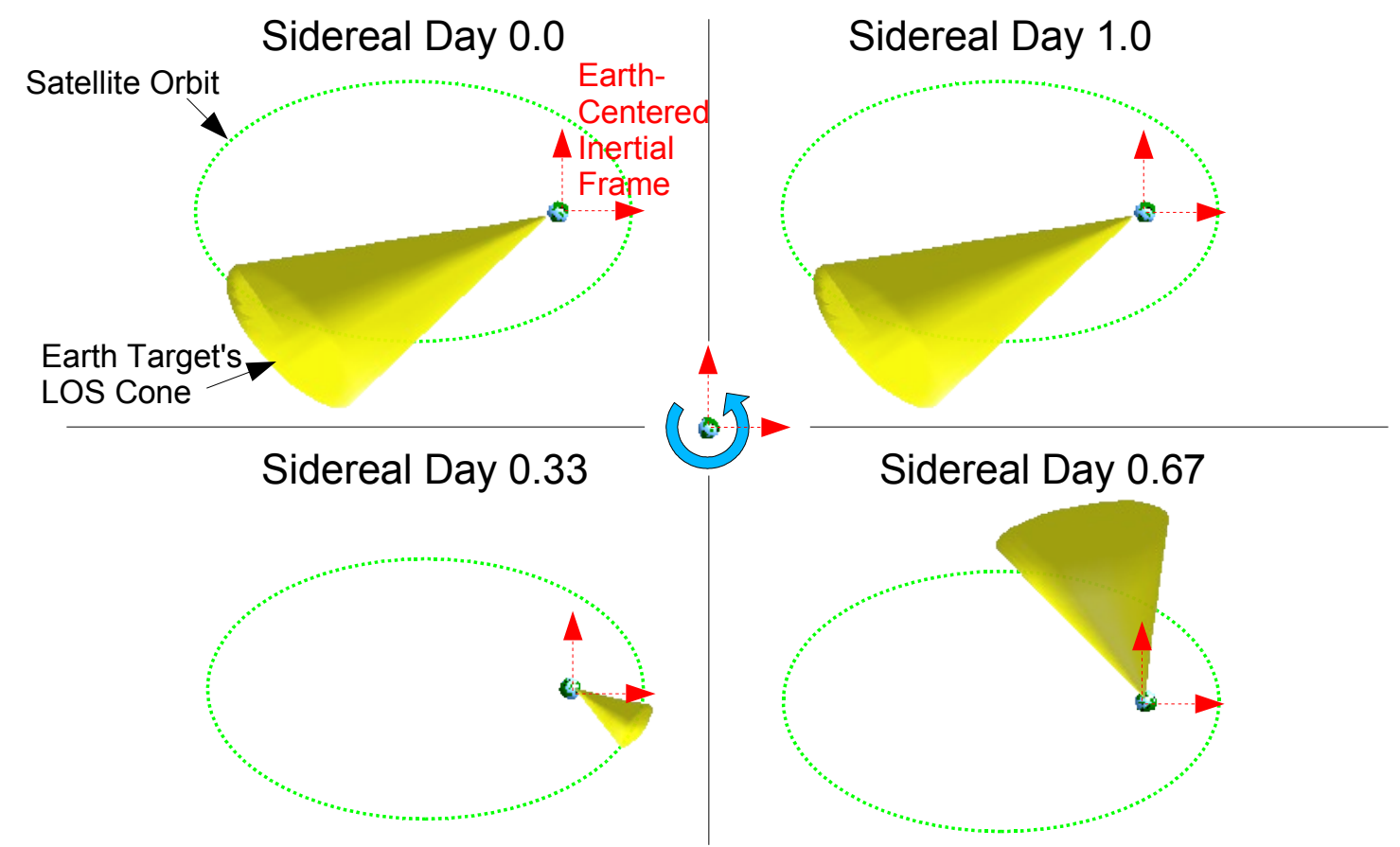

Figure 5: A qualitative depiction of an LOS cone emanating from a target on the surface of the Earth. The portion of the satellite orbit that is instantaneously visible from the target is the portion that intersects the LOS cone. Since the Earth revolves around an Earthcentered inertial frame once per sidereal day (i.e. approximately 23 hours 56 minutes), both the target and its LOS cone revolve with it. Therefore, the portion of the satellite orbit that is visible from a target also changes during the sidereal day.

However, the portion of a satellite orbit visible from a target is not static in time. Figure 5 depicts an LOS cone emanating from a target on the surface of

C This was partly inspired by Luke Sauter's "volumetric coverage" work in the context of satellite constellation design for mid-course ballistic missile interception ${ }^{38}$. 
the Earth. Since the Earth revolves around an Earth-centered inertial frame once per sidereal day (i.e. approximately 23 hours 56 minutes), both the target and its LOS cone revolve with it. Therefore, the portion of the satellite orbit that is visible from a target also changes during the sidereal day. Furthermore, the amount of time required by a satellite to traverse different segments of an orbit depends on both the length of the segment and on the nonlinear effect of gravity (see Section 3.3).

How to estimate coverage will be presented in three steps:

1. Determining the view of a satellite orbit from any point on the Earth (Section 3.2)

2. Determining the amount of time required for a satellite to traverse a segment of the satellite orbit (Section 3.3)

3. Estimating the satellite coverage of a target revolving in the Earthcentered inertial frame based on its changing view of a satellite orbit (Section 3.5)

The assumptions for the following analysis are:

(a) The Earth is a perfect sphere.

(b) No other celestial bodies, aside from the Earth, affect the motion of a satellite.

(a) Over the course of any sidereal day, satellite motion is modeled as in the classical two-body problem ${ }^{39}$ where the Earth is modeled as a point-mass and where the satellite has a much smaller mass. This is a first-order model of Earth's gravity.

(b) Over the course of multiple sidereal days, the second-order gravity effects of the Earth (see Section 3.5.2: Multiple-Day, Single-Satellite Coverage Estimation) will be treated as instantaneous changes to the orientation of a satellite orbit.

(c) The effect of atmospheric drag on the motion of the satellites is insignificant for the timespan and orbit types being considered and can, therefore, be ignored.

(d) A target on the surface of the Earth has a minimum elevation angle below which satellites are not visible.

(e) Line-of-sight is not hindered by atmospheric conditions (e.g. clouds, lighting conditions, etc.).

(f) There is no limit on the ability of a satellite to skew its orientation.

(g) If a target on the surface of the Earth can view a satellite then the satellite can also view the target (i.e. ignore the orientation of the satellite). 


\section{1: Classical Representation of an Orbit}

Before discussing how satellite coverage can be estimated, a brief discussion of the representation of an orbit is necessary. Developed by Kepler ${ }^{1}$, the classical representation of an orbit is shown in Figs. 6 and 7 and described in Table 3.

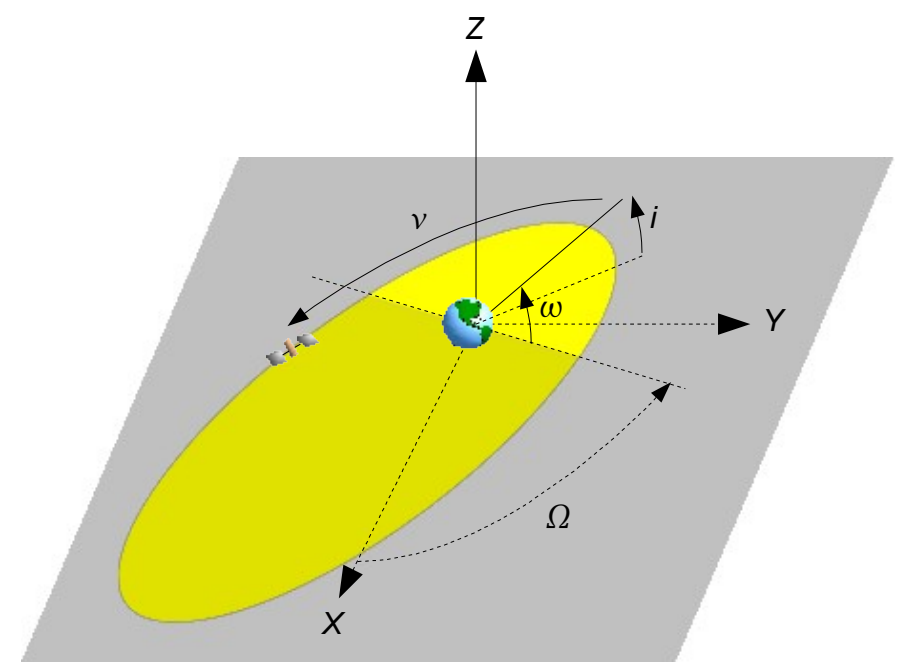

Figure 6: Depiction of a Keplerian orbit and its orbital elements within an Earth-centered inertial frame.

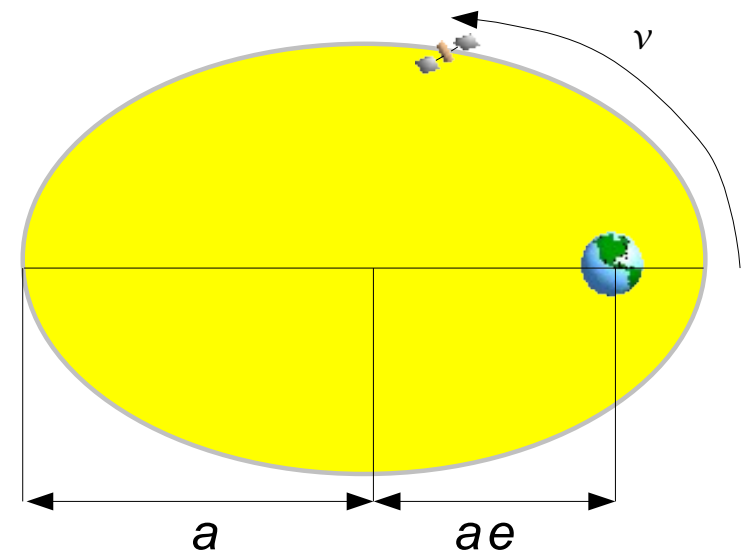

Figure 7: Depiction of a Keplerian orbit and its in-plane orbital elements.

Table 3: Descriptions of the classical orbital elements

\begin{tabular}{|c|l|}
\hline $\begin{array}{c}\text { Keplerian } \\
\text { Orbital } \\
\text { Element }\end{array}$ & \multicolumn{1}{|c|}{$(\mathbf{L U}=$ Length Unit, TU = Time Unit, deg = angular degrees) } \\
\hline$a$ & Semi-major axis $(\mathrm{SMA}): a \geq 0[L U]$ \\
\hline$e$ & $\begin{array}{l}\text { Eccentricity: } \mathrm{e}=0=\text { circular orbit; } 0<\mathrm{e}<1=\text { elliptical orbit; } \mathrm{e}=1= \\
\text { parabolic orbit; } \mathrm{e}>1=\text { hyperbolic orbit }\end{array}$ \\
\hline
\end{tabular}




\begin{tabular}{|c|c|}
\hline $\begin{array}{l}\text { Keplerian } \\
\text { Orbital } \\
\text { Element }\end{array}$ & $\begin{array}{c}\text { Description } \\
\text { (LU = Length Unit, } \mathrm{TU}=\text { Time Unit, deg = angular degrees })\end{array}$ \\
\hline$i$ & Inclination: $0[\mathrm{deg}] \leq i \leq 180[\mathrm{deg}]$ \\
\hline$\Omega$ & $\begin{array}{l}\text { Right-ascension of the ascending node (RAAN): } 0[\mathrm{deg}] \leq \Omega \leq 360[\mathrm{deg}] \\
\text { valid for inclined orbits; not defined for equatorial orbits }\end{array}$ \\
\hline$\omega$ & $\begin{array}{l}\text { Argument of periapsis: } 0[\mathrm{deg}] \leq \omega \leq 360[\mathrm{deg}] \text { valid for inclined, elliptical } \\
\text { orbits; not defined otherwise }\end{array}$ \\
\hline$v$ & True anomaly: $0[\mathrm{deg}] \leq v \leq 360[\mathrm{deg}]$ \\
\hline M & $\begin{array}{l}\text { Mean anomaly: } 0[\mathrm{deg}] \leq M \leq 360[\mathrm{deg}] \text { (See 3.4.4: Earth Rotates } \\
\text { Through a Virtual Inertial Sphere) }\end{array}$ \\
\hline$\dot{M}$ & $\begin{array}{l}\text { Mean orbital rate of rotation: } \dot{M} \geq 0\left[\frac{1}{T U}\right] ; \text { equal to the rate-of-change } \\
\text { of the Mean Anomaly, } \frac{d M}{d t}\end{array}$ \\
\hline$\vec{h}$ & $\begin{array}{l}\text { Angular momentum vector of the satellite: }|\vec{h}| \geq 0\left[\frac{L U^{2}}{T U}\right] \text {. Equal to the } \\
\text { cross-product of the satellite's Earth-centered position, } \vec{r} \text {, with the } \\
\text { satellite's Earth-centered velocity, } \vec{v} \text {. }\end{array}$ \\
\hline
\end{tabular}

The radial distance of a satellite from the center of the Earth is given by

$$
r=\frac{a\left(1-e^{2}\right)}{1+e \cos (v)}
$$

The angular momentum of the satellite is defined as

$$
\vec{h}=\vec{r} \times \vec{v},
$$

where $\vec{r}$ is the satellite's Earth-centered position, and $\vec{v}$ is the satellite's Earthcentered velocity.

Some angles are defined to handle special orbits that cause certain Keplerian orbital elements to not be defined. In the case of a circular, equatorial orbit, the orbital plane is coplanar with the plane formed by the X-and Y-axes (See Fig. 9). Within this plane, the angular position of a satellite measured counter-clockwise from the X-axis is called the "true longitude", $\lambda_{\text {true }}$. 


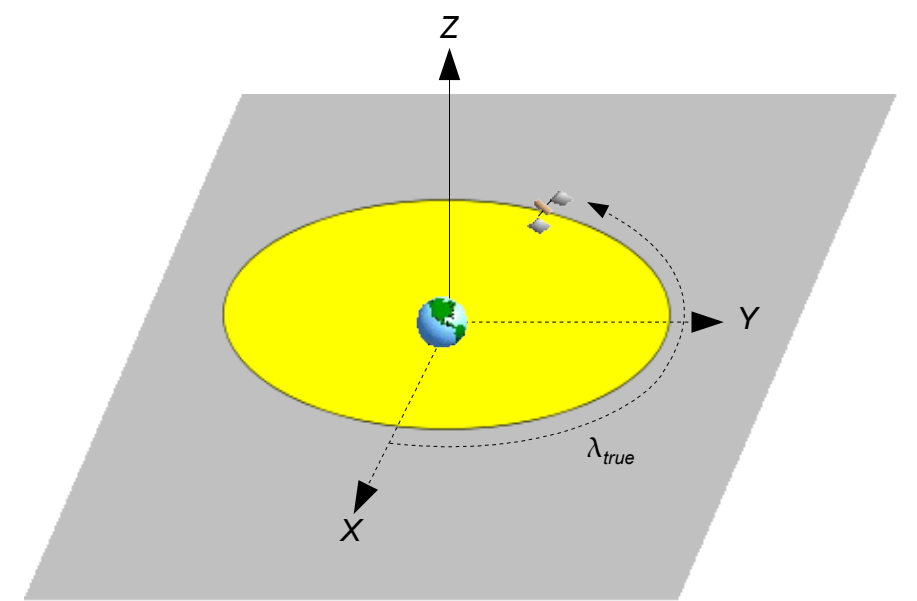

Figure 8: Depiction of a circular, equatorial orbit within an Earth-centered inertial frame.

In the case of an elliptical, equatorial orbit, the orbital plane is coplanar with the plane formed by the $X$ - and $Y$-axes (See Fig. 9). Within this plane, the angular position of periapsis measured counter-clockwise from the $X$-axis is called the "true longitude of periapsis", $\omega_{\text {true }}$.

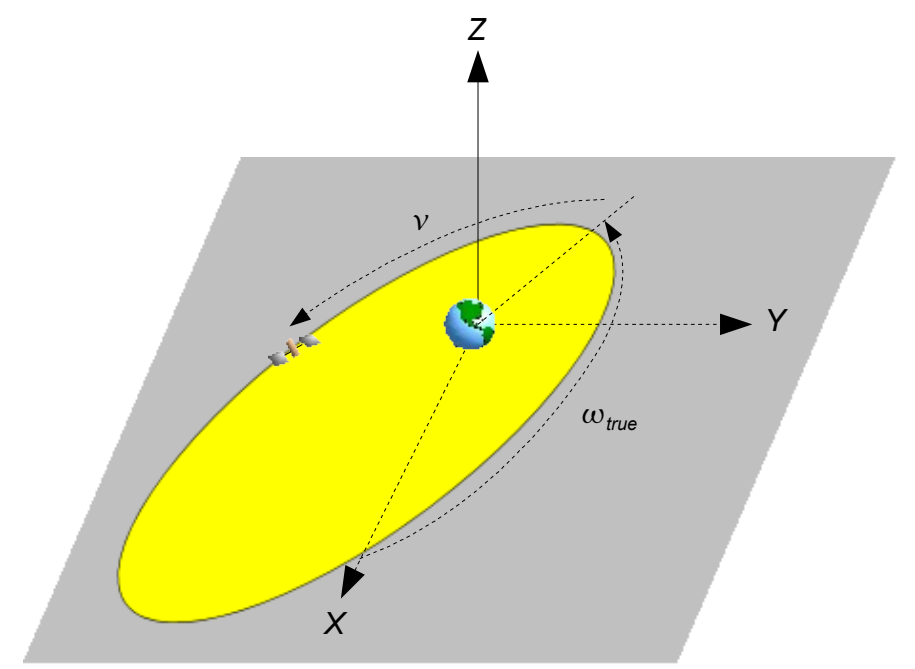

Figure 9: Depiction of an elliptical, equatorial orbit within an Earth-centered inertial frame.

In the case of a circular, inclined orbit, the angular position of a satellite along its orbit is measured from the ascending node within the orbital plane and is called the "argument of latitude", u (See Fig. 10). 


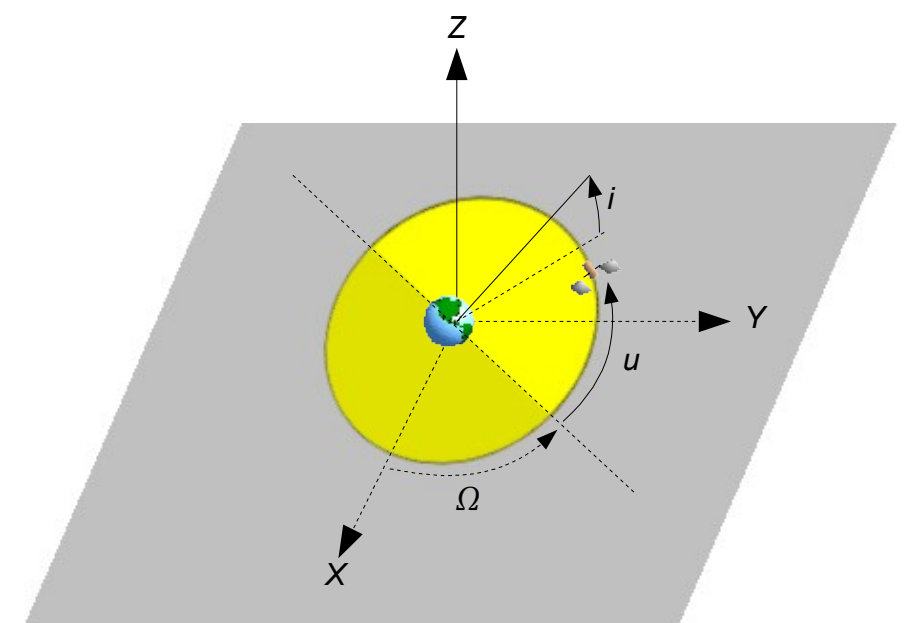

Figure 10: Depiction of a circular, inclined orbit within an Earth-centered inertial frame.

\section{2: Determining the View of a Satellite Orbit from a Point on a Sphere}

The first step towards estimating coverage is to identify what portion of a satellite orbit can be seen from the surface of an Earth-centered sphere whose radius equals one Earth radius $(E R)^{D}$ (see Fig. 11). From any point on this sphere, a zenith-pointing, LOS cone is projected outwards. The angle made between the edge of the LOS cone and the target's local horizon is specified by a minimum-elevation angle, el, below which a target's view of the sky is obstructed.

This section focuses on identifying where a target's LOS cone intersects with a satellite's orbit. The satellite orbit/ellipse is defined by the instantaneous position and velocity of a satellite relative the Earth (see Section 3.1: Classical Representation of an Orbit). The satellite can be situated anywhere along the orbit. Therefore, it is worth emphasizing to the reader that when the intersection of the LOS cone with the satellite orbit is determined, no assertion is being made as to whether the satellite happens to be situated along that segment of the orbit. Dealing with whether a satellite traverses the orbital segment in view is left for Section 3.5: Estimating Coverage from Information across a Target's Latitude.

D Note that the work in this section is equally applicable to other celestial bodies that are nearly spherical. 


\subsection{1: Where does the LOS Cone intersect with the orbital plane?}

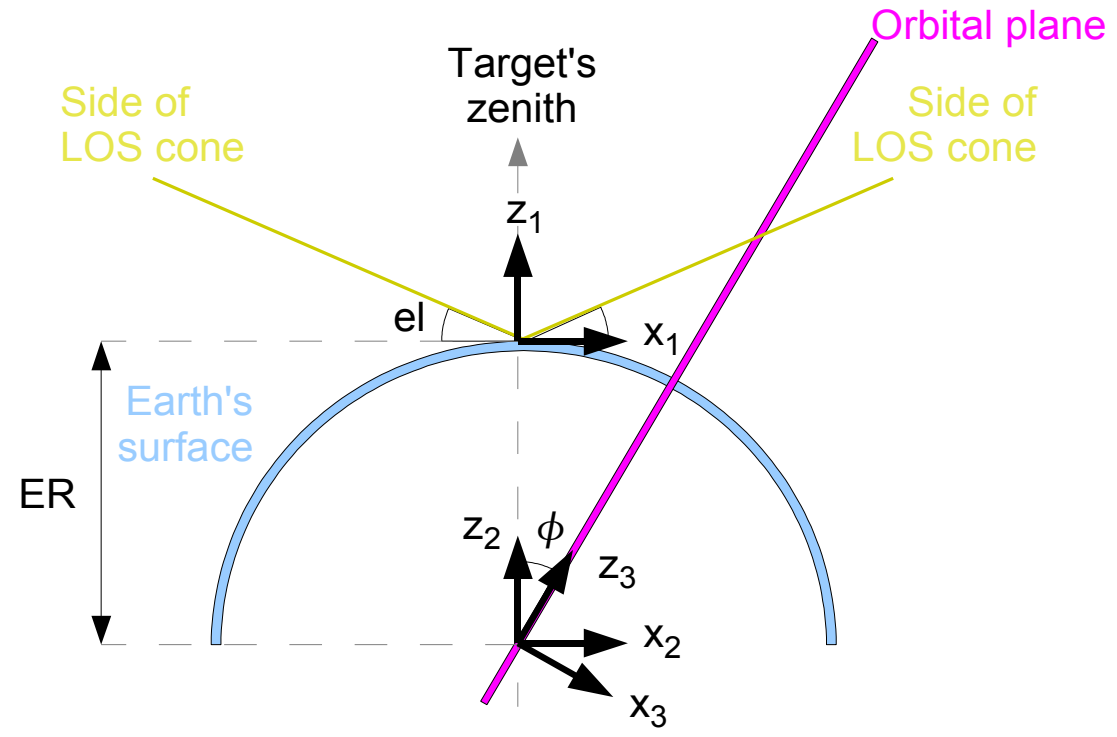

Figure 11: Depiction of an observer's LOS cone emanating from the surface of the Earth and its intersection with the orbital plane.

The equation for the LOS cone in Frame 1 is

$$
z_{1}^{2}=A\left(x_{1}^{2}+y_{1}^{2}\right),
$$

where

$$
A=\tan ^{2}(e l) .
$$

It is desirable to express this equation in terms of Frame 3 coordinates.

Let

$$
\phi=90^{\circ}-\operatorname{acos}\left(\hat{z}_{1} \cdot \hat{h}\right),
$$

where $\hat{h}$ is the unit angular momentum vector of the satellite. The angular momentum vector is also the normal of the orbital plane.

The expression of Frame 1 in terms of Frame 2 is

$$
\left[\begin{array}{c}
x_{1} \\
y_{1} \\
z_{1}
\end{array}\right]=\left[\begin{array}{c}
x_{2} \\
y_{2} \\
z_{2}-E R
\end{array}\right]
$$

The expression of Frame 2 in terms of Frame 3 is

$$
\left[\begin{array}{l}
x_{2} \\
y_{2} \\
z_{2}
\end{array}\right]=\vec{b}_{2}=\operatorname{Rot}_{y}(-\phi) \vec{b}_{3}=\left[\begin{array}{ccc}
c^{-} \phi & 0 & -s^{-} \phi \\
0 & 1 & 0 \\
s^{-} \phi & 0 & c^{-} \phi
\end{array}\right]\left[\begin{array}{l}
x_{3} \\
y_{3} \\
z_{3}
\end{array}\right]=\left[\begin{array}{c}
c^{-} \phi x_{3}-s^{-} \phi z_{3} \\
y_{3} \\
s^{-} \phi x_{3}+c^{-} \phi z_{3}
\end{array}\right],
$$


where, for brevity,

$$
\begin{aligned}
& c \phi=\cos (+\phi) \\
& c^{-} \phi=\cos (-\phi) \\
& s \phi=\sin (+\phi) \\
& s^{-} \phi=\sin (-\phi) .
\end{aligned}
$$

The expression of Frame 1 in terms of Frame 3 is

$$
\left[\begin{array}{c}
x_{1} \\
y_{1} \\
z_{1}
\end{array}\right]=\left[\begin{array}{c}
c^{-} \phi x_{3}-s^{-} \phi z_{3} \\
y_{3} \\
s^{-} \phi x_{3}+c^{-} \phi z_{3}-E R
\end{array}\right] .
$$

Therefore, the equation for the LOS cone, Eq. (7), in terms of Frame 3 is

$$
\left(s^{-} \phi x_{3}+c^{-} \phi-E R\right)^{2}=A\left(c^{-} \phi x_{3}-s^{-} \phi z_{3}\right)^{2}+A y_{3}^{2} \text {. }
$$

To identify where this cone intersects the orbital plane, simply let

$$
x_{3}=0 \text {. }
$$

Then Eq. (9) becomes

$$
\left(c^{-} \phi-E R\right)^{2}=A\left(-s^{-} \phi z_{3}\right)^{2}+A y_{3}^{2} \text {. }
$$

Squaring the terms leads to

$$
\cos ^{2}(-\phi) z_{3}^{2}+E R^{2}-2 E R \cos (-\phi) z_{3}=A-\sin ^{2}(-\phi) z_{3}^{2}+A y_{3}^{2} \text {. }
$$

Re-arranging leads to

$$
A y_{3}^{2}+B z_{3}^{2}+C z_{3}=E R^{2},
$$

where

$$
\begin{aligned}
B & =A \sin ^{2}(-\phi)-\cos ^{2}(-\phi), \\
\text { and } \quad C & =2 E R \cos (-\phi) .
\end{aligned}
$$

Eq. (10) defines the locus of points defining the LOS cone intersection with the orbital plane that was determined relative to Frame 3.

\subsection{2: Intersection of the LOS cone with the orbital ellipse}

The orbital ellipse is located within the same orbital plane, and is traditionally defined with respect to Frame 5 (see Fig. 13). Therefore, the locus of points will be re-expressed with respect to Frame 5 in order to identify the intersection in terms of the true anomaly of the ellipse.

Figure 12 depicts the orientation of Frame 4 with respect to the surface of the Earth and the orbital plane. Its orientation is dependent on the direction of the unit angular momentum vector, $\hat{h}$, in order to accommodate posigrade 
$\left(0 \leq i<\frac{\pi}{2}\right)$ and retrograde $\left(\frac{\pi}{2}<i \leq \pi\right)$ orbits, and the eventual transformation to Frame 5. The definition of Frame 4's Cartesian directions are

$$
\begin{aligned}
& \hat{x}_{4}=-\hat{h}, \\
& \hat{z}_{4}=\hat{z}_{3}=\text { unit }\left[\hat{z}_{1}-\text { unit }\left\{\left(\hat{z_{1}} \cdot \hat{h}\right) \cdot \hat{h}\right\}\right], \text { and } \\
& \hat{y}_{4}=\hat{z}_{3} \times \hat{x}_{3},
\end{aligned}
$$

where the "unit" function normalizes the magnitude of the vector to one.
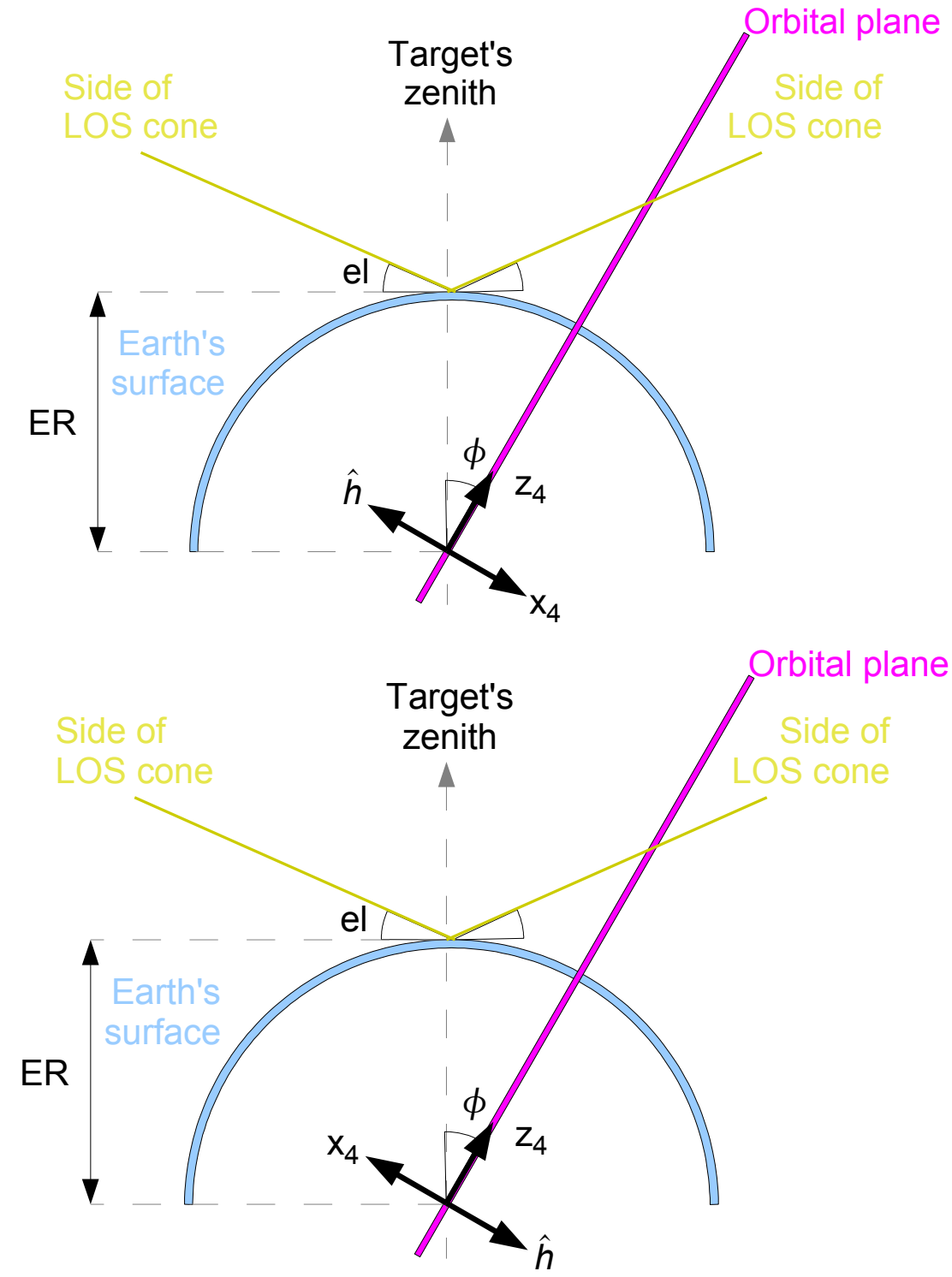

Figure 12: Depiction of Frame 4 and its dependence on the angular momentum vector of the satellite, $\hat{h}$. 


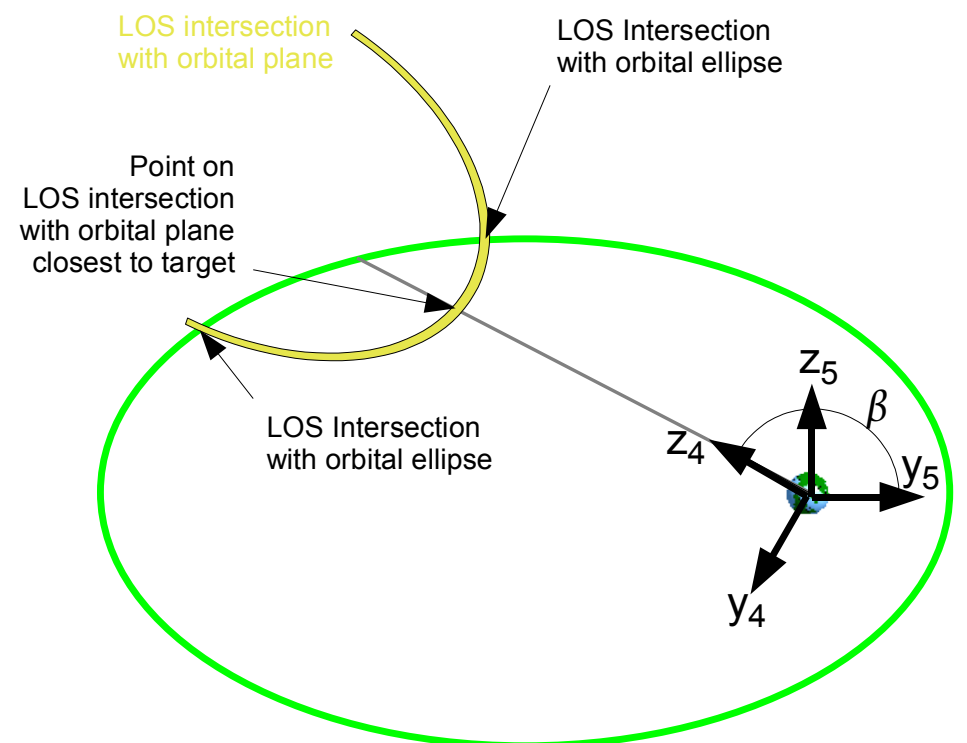

Figure 13: Depiction of the intersection of the LOS cone with the orbital plane.

The expression of Frame 4 in terms of Frame 5 is

$$
\left[\begin{array}{c}
z_{4} \\
y_{4} \\
x_{4}
\end{array}\right]=\vec{b}_{4}=\operatorname{Rot}_{z}(-\beta) \vec{b}_{5}=\left[\begin{array}{ccc}
c^{-} \beta & s^{-} \beta & 0 \\
-s^{-} \beta & c^{-} \beta & 0 \\
0 & 0 & 1
\end{array}\right]\left[\begin{array}{c}
x_{5} \\
y_{5} \\
z_{5}
\end{array}\right]=\left[\begin{array}{c}
c^{-} \beta x_{5}+s^{-} \beta y_{5} \\
-s^{-} \beta x_{5}+c^{-} \beta y_{5} \\
z_{5}
\end{array}\right],
$$

where

$$
\beta=\operatorname{acos}\left(\hat{z}_{4} \cdot \hat{e}\right) \operatorname{sign}\left(\hat{y}_{4} \cdot \hat{e}\right),
$$

and where $\hat{e}$ is the unit direction of the eccentricity vector (i.e. pointing toward periapsis).

Then, re-phrasing Eq. (10) in terms of Frame 5 results in

$$
\begin{aligned}
A\left\{\sin ^{2}(-\beta) x_{5}^{2}+\cos ^{2}(-\beta) y_{5}^{2}-2 \cos (-\beta) \sin (-\beta) x_{5} y_{5}\right\} \\
\quad+B\left\{\cos ^{2}(-\beta) x_{5}^{2}+\sin ^{2}(-\beta) y_{5}^{2}+2 \cos (-\beta) \sin (-\beta) x_{5} y_{5}\right\} \\
\quad+C\left\{\cos (-\beta) x_{5}+\sin (-\beta) y_{5}\right\} \\
\quad=E R^{2} .
\end{aligned}
$$

Recall that

$$
\begin{aligned}
& r=\frac{a\left(1-e^{2}\right)}{1+e \cos (v)}, \\
& x=r \cos (v)=\frac{a\left(1-e^{2}\right)}{1+e \cos (v)} \cos (v)=\frac{E}{F} \cos (v), \text { and }
\end{aligned}
$$




$$
y=r \sin (v)=\frac{a\left(1-e^{2}\right)}{1+e \cos (v)} \sin (v)=\frac{E}{F} \sin (v),
$$

where

$$
\begin{aligned}
& E=a\left(1-e^{2}\right) \text { and } \\
& F=1+e \cos (v) .
\end{aligned}
$$

Therefore, Eq. (13) can be re-expressed as

$$
\begin{aligned}
A E^{2} & \left\{\sin ^{2}(-\beta) \cos ^{2}(\nu)+\cos ^{2}(-\beta) \sin ^{2}(\nu)\right\} \\
& +A E^{2}\left\{-2 \cos (-\beta) \sin (-\beta) \cos (\nu) \sin ^{2}(\nu)\right\} \\
& +B\left\{\cos ^{2}(-\beta) \cos ^{2}(\nu)+\sin ^{2}(-\beta) \sin ^{2}(\nu)\right\} \\
& +B\{+2 \cos (-\beta) \sin (-\beta) \cos (\nu) \sin (\nu)\} \\
& +C E F\{\cos (-\beta) \cos (\nu)+\sin (-\beta) \sin (v)\} \\
& =E R^{2} F^{2} .
\end{aligned}
$$

Finding the value of $v$, the true anomaly, that satisfies this equation can be performed using a numerical root-finding algorithm. There are up to four roots to this equation. The determination of the desirable root depends on placing appropriate lower and upper bounds during the root-finding process (thus requiring the use of a bracketing method for the root-finding). This is discussed in the following section.

\subsection{3: What are the bounds of the intersection of the LOS cone with the orbital ellipse?}

As depicted in Fig. 13, the intersections of the LOS cone with the orbital ellipse are on either side of the line within the orbital plane that contains the closest LOS intersection. That line is used as the lower bound when numerically seeking the "left" intersection of the LOS cone with the orbital plane; the same line will be used as an upper bound when numerically seeking the "right" intersection. The true anomaly of that line, $v_{c}$, is equal to the negative value of $\beta$ from Eq. (12),

$$
v_{C}=-\beta=-\operatorname{acos}\left(\vec{z}_{4} \cdot \vec{e}\right) \operatorname{sign}\left(\vec{y}_{4} \cdot \vec{e}\right)
$$




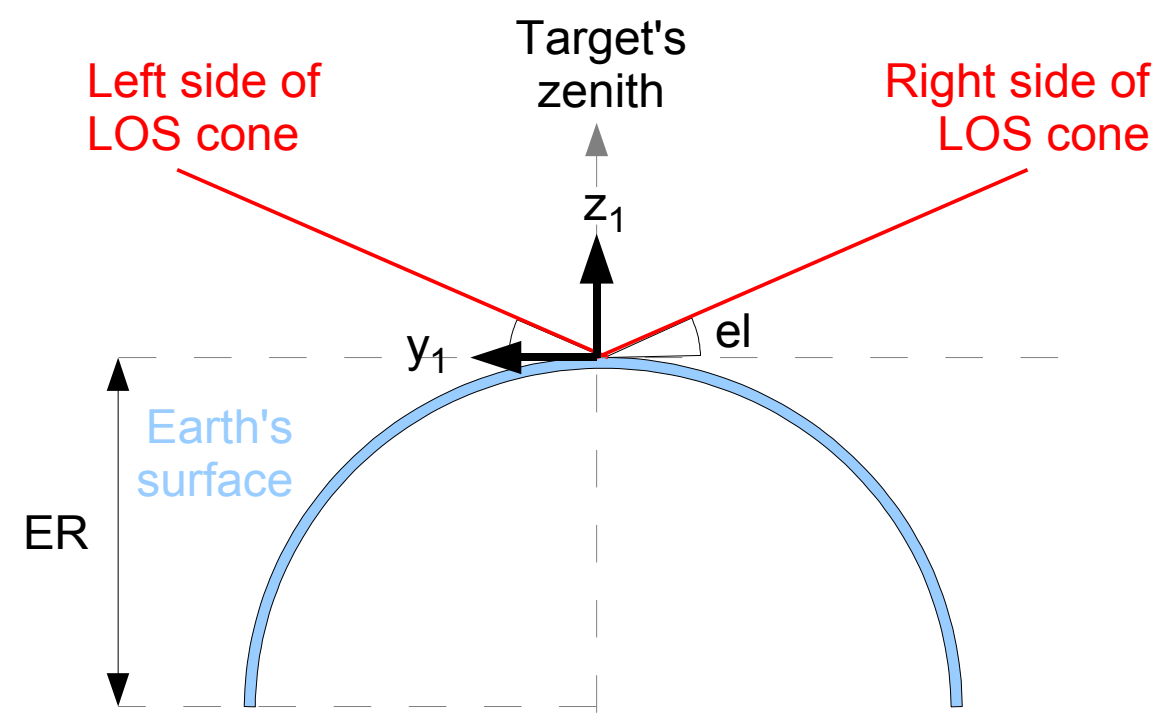

Figure 14: Depiction of an observer's LOS cone emanating from the surface of the Earth.

To obtain an upper bound on the left intersection, consider the geometry shown in Figs. 11, 14, and 15. The left side of the LOS cone is bounded by a three-dimensional plane that is tangent to the side of the LOS cone and is also perpendicular to the orbital plane. Similarly, there is a "right-side" plane. These planes intersect the orbital plane and provide a bound on the extent of the intersection of the LOS cone with the orbital plane.

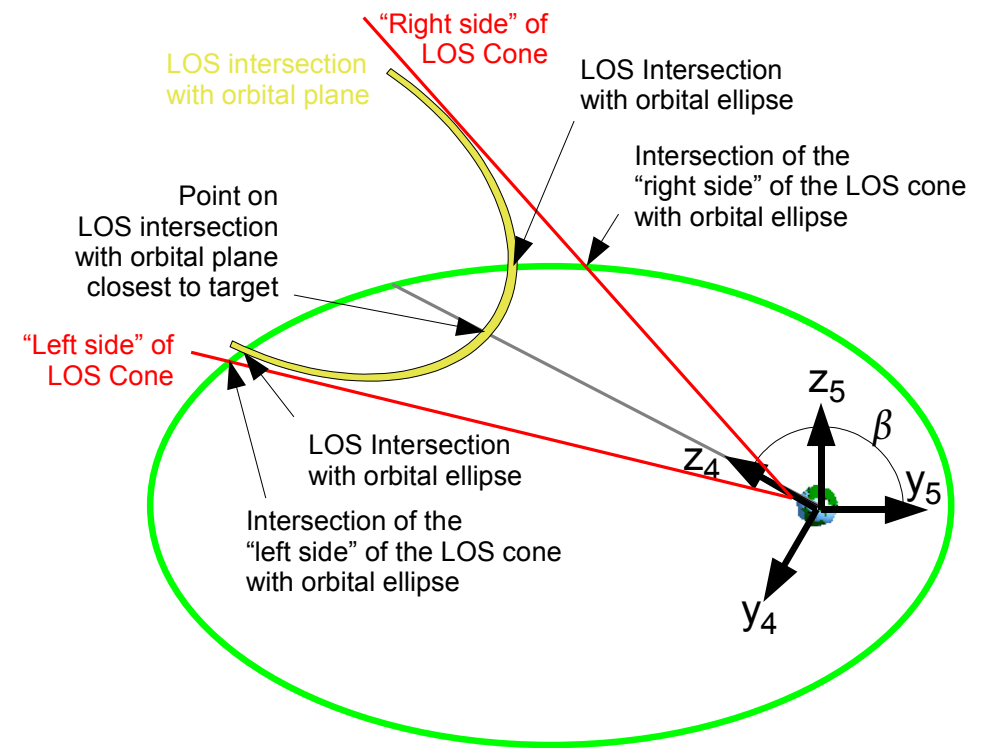

Figure 15: Depiction of the intersection of the planar "sides" of the LOS cone with the orbital plane.

The equations for the planes that bound the sides of the LOS cone can be described by

Left Plane $-\sin (e l) y_{1}+\cos (e l) z_{1}=0$ and 
Right Plane $\sin (e l) y_{1}+\cos (e l) z_{1}=0$.

\section{Where do the planes intersect the orbital plane?}

Using the transformation from Frame 1 to Frame 5 represented by Eq. (8) and Eq. (11) results in

Left Plane $\quad J_{L} x_{5}+K_{L} y_{5}=L$ and

Right Plane $J_{R} x_{5}+K_{R} y_{5}=L$,

where

$$
\begin{aligned}
& J_{L}=\sin (e l) \sin (-\beta)+\cos (-\phi) \cos (e l) \cos (-\beta), \\
& J_{R}=-\sin (e l) \sin (-\beta)+\cos (-\phi) \cos (e l) \cos (-\beta), \\
& K_{L}=-\sin (e l) \cos (-\beta)+\cos (-\phi) \cos (e l) \sin (-\beta), \\
& K_{R}=\sin (e l) \cos (-\beta)+\cos (-\phi) \cos (e l) \sin (-\beta), \text { and } \\
& L=\cos (e l) E R .
\end{aligned}
$$

Where does the intersection of the planes with the orbital plane intersect the orbital ellipse?

Solving for $y_{5}$ results in

Left Plane $\quad y_{5}=\frac{-J_{L}}{K_{L}} x_{5}+\frac{L}{K_{L}}$ and

Right Plane $y_{5}=\frac{-J_{R}}{K_{R}} x_{5}+\frac{L}{K_{R}}$.

Re-expressing $x_{5}$ and $y_{5}$ in terms of the true anomaly, $v$, by using Eq. (14) yields

Left Plane $E \sin (v)=\cos (v) M_{L}+N_{L}$ and

Right Plane $E \sin (v)=\cos (v) M_{R}+N_{R}$, where

$$
\begin{aligned}
& M_{L}=\frac{-J_{L}}{K_{L}} E+\frac{L}{K_{L}} e, \\
& M_{R}=\frac{-J_{R}}{K_{R}} E+\frac{L}{K_{R}} e, \\
& N_{L}=\frac{L}{K_{L}}, \text { and } \\
& N_{R}=\frac{L}{K_{R}} .
\end{aligned}
$$


Squaring both sides of Eq. (17) and using the trigonometric identity

$$
\sin ^{2}(a)+\cos ^{2}(a)=1
$$

yields

Left Plane $\cos ^{2}(v)\left\{M_{L}^{2}+E^{2}\right\}+\cos (v)\left\{2 M_{L} N_{L}\right\}+\left\{N_{L}^{2}-E^{2}\right\}=0$ and

Right Plane $\cos ^{2}(v)\left\{M_{R}^{2}+E^{2}\right\}+\cos (v)\left\{2 M_{R} N_{R}\right\}+\left\{N_{R}^{2}-E^{2}\right\}=0$.

Re-arranging the equations and solving for the multi-valued term, $\cos \left(v_{1,2}\right)$, yields

Left Plane $\quad \cos \left(v_{1,2}\right)=\frac{-M_{L} N_{L} \pm E \sqrt{M_{L}^{2}+E^{2}-N_{L}^{2}}}{M_{L}^{2}+E^{2}}$ and

Right Plane $\cos \left(v_{1,2}\right)=\frac{-M_{R} N_{R} \pm E \sqrt{M_{R}^{2}+E^{2}-N_{R}^{2}}}{M_{R}^{2}+E^{2}}$.

This is almost sufficient to determine the angle. However, there is still an ambiguity regarding which of the two angles, $v_{1}$ or $v_{2}$, is the physically correct result. Some additional calculations clarify the situation.

Using Eqs. (5) and (19), one can find a value for the radius,

$$
r=\frac{a\left(1-e^{2}\right)}{1+e \cos \left(v_{1,2}\right)} \text {. }
$$

Solve for $x$ using Eq. (14a),

$$
x_{5}=r \cos \left(v_{1,2}\right) \text {. }
$$

Solve for y using Eq. (17),

Left Plane $\quad y_{5}=\frac{-J_{L}}{K_{L}} x_{5}+\frac{L}{K_{L}}$ and

Right Plane $y_{5}=\frac{-J_{R}}{K_{R}} x_{5}+\frac{L}{K_{R}}$.

Having values for both $\mathrm{x}$ and $\mathrm{y}$ now resolves any ambiguity regarding where the side of the LOS cone intersects the orbital ellipse.

Special Condition: $\mathrm{K}=0$

In the event that either $K L$ or $K_{R}$ equals 0 , then Eq. (16) provides a value for $x_{5}$, Left Plane $\quad x_{5}=\frac{L}{J_{L}}$ and Right Plane $\quad x_{5}=\frac{L}{J_{R}}$. 
The value for $x_{5}$ is also defined by Eq. (14a). By setting these two definitions equal to each other, a value for the true anomalies that satisfy these equations can be found:

Left Plane

$$
\begin{aligned}
& v_{1}=\operatorname{acos}\left(\frac{x_{5}}{a\left(1-e^{2}\right)-e x_{5}}\right)=\operatorname{acos}\left(\frac{\frac{L}{J_{L}}}{a\left(1-e^{2}\right)-e \frac{L}{J_{L}}}\right), \\
& v_{2}=-v_{1},
\end{aligned}
$$

$\underline{\text { Right Plane }}$

$$
\begin{aligned}
& v_{1}=\operatorname{acos}\left(\frac{x_{5}}{a\left(1-e^{2}\right)-e x_{5}}\right)=\operatorname{acos}\left(\frac{\frac{L}{J_{R}}}{a\left(1-e^{2}\right)-e \frac{L}{J_{R}}}\right), \text { and } \\
& v_{2}=-v_{1} .
\end{aligned}
$$

\subsection{4: When can it be assured that the orbital plane will not be visible from a target?}

There are certain geometries of the target relative to the orbital ellipse where an LOS cone is assured of not intersecting the orbit. If this can easily be determined then the calculations from the previous two sections can be avoided as irrelevant. When considering Fig. 13, one shortcut is to determine whether the LOS cone intersection with the orbital plane is far beyond the farthest reach of the satellite orbit (i.e. its apoapsis). When considering Fig. 11, it is evident that for values of $\phi$ approaching 90 degrees, the orbital ellipse will not be visible at all from the target ${ }^{\mathrm{E}}$. The point of closest approach to the center of the sphere (which is assumed to be collocated with the Earth) occurs when $y_{4}=0$. Manipulating Eq. (10) and solving for $z_{4}$ results in a quadratic equation; its solution is

$$
z_{4}=E R\left\{\frac{-\cos (-\phi)+\sqrt{A} \sin (-\phi)}{A \sin ^{2}(\phi)-\cos ^{2}(\phi)}\right\},
$$

where one of the two solutions has been removed due to its being beneath the target. This point of closest approach can then be compared to the point of furthest deviation from the Earth, namely the apoapsis. The apoapsis, which is defined to be at $v=\pi$, can be calculated from Eq. (5) to be

$$
r_{a}=a(1+e) \text {. }
$$

$\mathrm{E}$ Note that the value of $\phi$ is effectively a target's spherical latitude relative to the orbital plane. This orientation of the sphere relative to the orbital ellipse will be discussed further in Section 3.4.1: Virtual Sphere Aligned with Satellite Orbit. 
If $z_{4}>r_{a}$, then it is impossible for the LOS cone to intersect with the orbital ellipse; this condition preempts the need to calculate the view of the orbital ellipse. Furthermore, this check can be used to identify the range of values for $\phi$ that are assured of having no visibility of the orbital ellipse.

\subsection{5: Is the closest intersection point closer than the ellipse's radius along the line of closest approach?}

Another shortcut can be taken to avoid calculating the visibility of the orbital ellipse from a point on the Earth's surface. The point of closest approach of the LOS cone lies along a line extending from the Earth outwards along the central true anomaly, $v_{C}$, defined by Eq. (15) (See Fig. 13). If this point is farther than the radius of the orbital ellipse defined at $v_{C}$ and calculated by Eq. (5), then the ellipse is not visible from that point on the sphere.

\section{3: Correlating Views of a Satellite Orbit to Time along an Orbit}

The previous section showed how to determine the portion of a satellite orbit in view from a target on the Earth's surface. That portion of the satellite orbit was identified by two points along the orbit: $v_{1}$ and $v_{2}$. This section addresses how to compute the amount of time required for a satellite to travel between two points on an orbit.

Figure 16 depicts two positions along an orbital ellipse: $v_{1}$ and $v_{2}$. The time of flight between two points on an eccentric orbit is non-trivial because the rateof-change of the true anomaly, $v$, is not constant. And the rate-of-change of the true anomaly is not constant because of the nonlinear effect of gravity. The equation of motion for a satellite, as a classical two-body problem ${ }^{39}$, is

$$
\frac{d}{d t} \vec{r}(t)=\frac{-G M_{\text {Earth }} \hat{r},}{r^{2}(t)}
$$

where $\mathrm{G}$ is the universal gravitational constant, $M_{\text {Earth }}$ is the mass of the Earth, $\vec{r}$ is the vector from the center of the Earth to the satellite, $\hat{r}$ is the unit-vector of $\vec{r}$, and $r$ is the magnitude of $\vec{r}$. (The classical two-body problem assumes that the motion of a very small satellite is affected only by the Earth which is modeled as a point-mass. A higher-fidelity mass model for the Earth is discussed in Section 2.1: Motion of Satellites in Orbit.) 


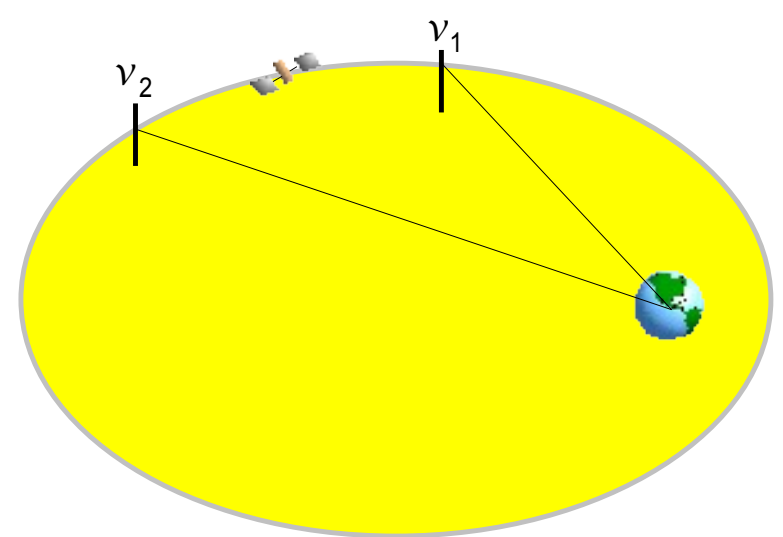

Figure 16: The time-of-flight to travel between two points on an elliptical orbit depends on the nonlinear effect of gravity.

Johannes Kepler solved this time-of-flight problem in two steps1 (see Fig. 17). In the first step, the angular position of the satellite along its orbital ellipse, $v$, is transformed in space onto an eccentric circle, with points identified by the eccentric anomaly, $E$,

$$
\tan \left(\frac{E}{2}\right)=\sqrt{\frac{1-e}{1+e}} \tan \left(\frac{v}{2}\right) .
$$

In the second step, the angular position along the eccentric circle, $E$, is transformed in time onto a mean circle with points identified by the mean anomaly, $M$. The relationship is

$$
M=n(t-T)=E-e \sin (E),
$$

where $n$ is the mean rate-of-motion (i.e. constant rate-of-change of the mean anomaly), $t$ is time, and $T$ is a reference time.

Within the mean circle, time-of-flight calculation is a linear calculation. Therefore, calculating the time-of-flight between $v_{1}$ and $v_{2}$ involves converting those true anomaly angles to their mean anomaly counterparts, $M_{1}$ and $M_{2}$, and then performing the following calculation:

$$
T O F=\frac{M_{2}-M_{1}}{n},
$$

where TOF is the time-of-flight. 


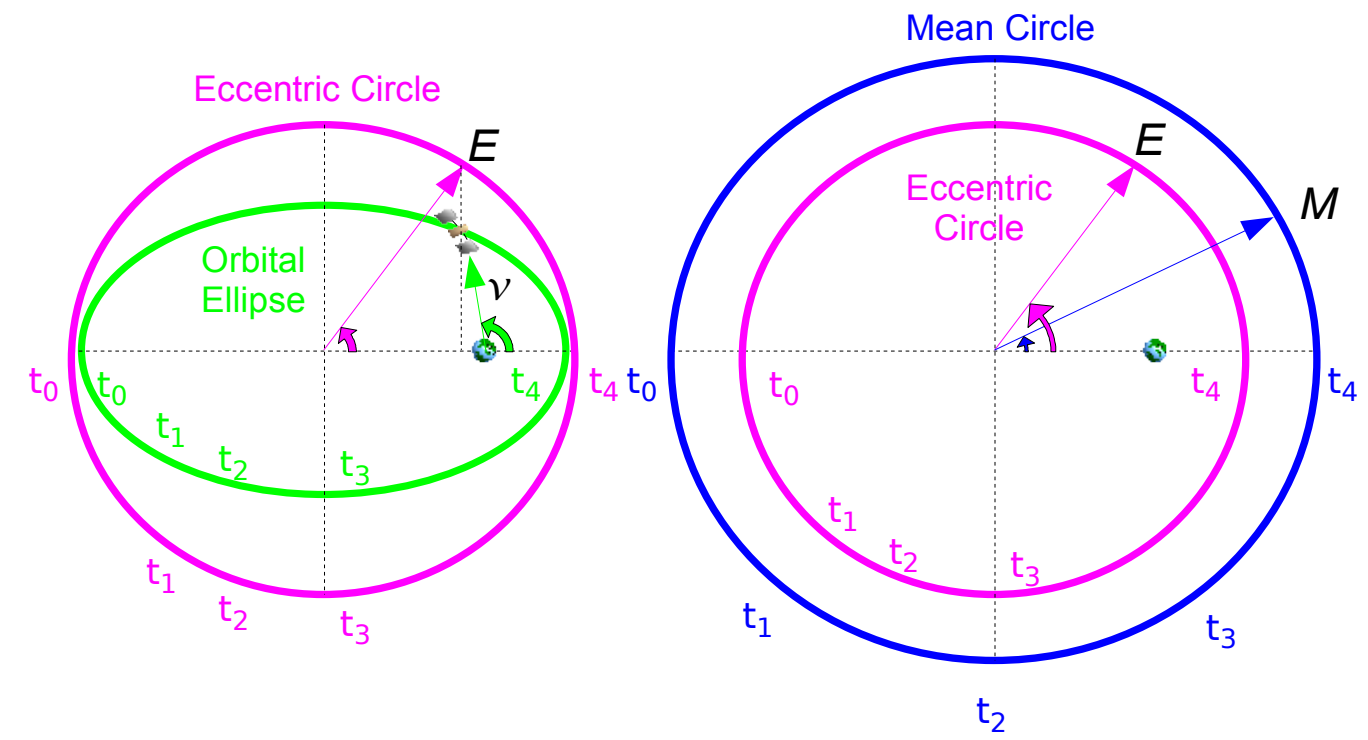

Figure 17: An orbital ellipse, its associated eccentric circle, and its associated mean circle.

\section{4: Earth-Centered Virtual Spheres}

This section discusses the use of two virtual spheres which, although not strictly necessary to estimate coverage, can be helpful in explaining the coverage provided by a satellite.

\subsection{1: Virtual Sphere Aligned with Satellite Orbit}

For every orbital ellipse, a virtual sphere can be imagined to be centered on the Earth yet aligned with the orbit. This sphere will be called the "orbit-fixed sphere". This sphere is independent of both the Earth-centered inertial frame and the Earth's surface (see Fig. 18). The satellite and its orbit-fixed sphere are, to first-order, independent of the orientation and rotation of the Earth's surface. The second-order dependence will be discussed in greater detail in Section 3.5.2: Multiple-Day, Single-Satellite Coverage Estimation. 

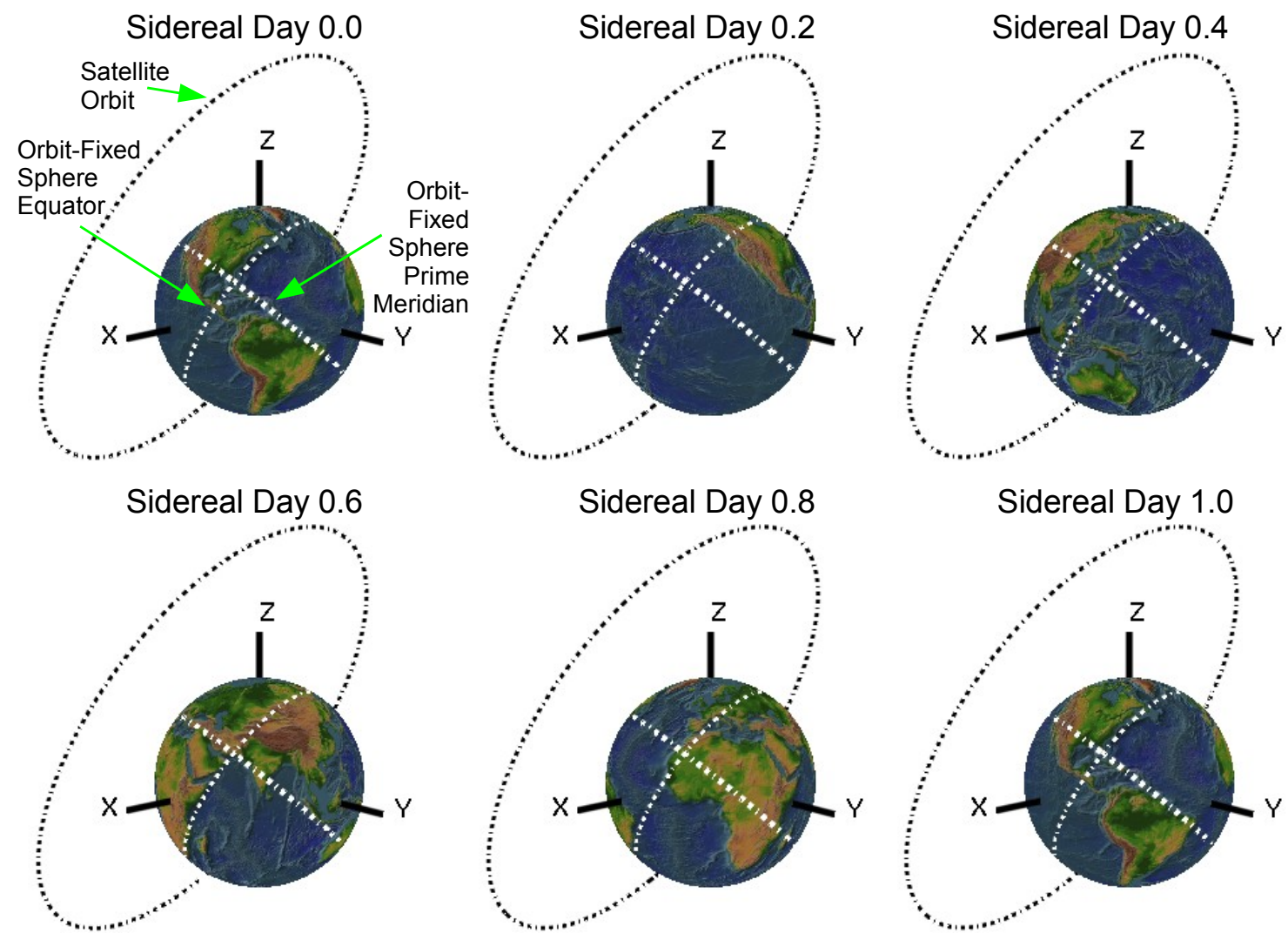

Figure 18: Depiction of a satellite orbit around the Earth over the course of a sidereal day. The satellite orbit and the Earth are shown relative to a Cartesian, Earth-centered inertial frame denoted by the $X-Y-Z$ axes. Note how the Earth rotates relative to the inertial frame. Although the Earth's second-order gravity effects cause the satellite orbit to twist relative to the inertial frame, it is a weak effect on this orbit and is hardly perceptible. The dotted white lines across the surface of the Earth are the great circles on the orbit-fixed sphere representing the orbit-fixed equator and the orbit-fixed prime meridian. Since the orbitfixed sphere is aligned with the satellite orbit, and since the satellite orbit barely moves relative to the inertial frame, the orbit-fixed equator and meridian also barely move.

\subsection{2: Visibility Map on the Orbit-Fixed Sphere}

For every point on the orbit-fixed sphere, the intersection between a zenithpointing LOS cone with the satellite's orbital ellipse can be determined. In so doing, the start and finish true anomalies of the intersection are identified. These true anomalies can then be converted to their mean anomaly counterparts by using Eqs. (21) and (22). Let a scalar representation of the visibility be defined by

$$
\text { visibility }=\frac{M_{\text {finish }}-M_{\text {start }}}{P},
$$

where $\mathrm{P}$ is the mean orbital period of the satellite orbit. The mean orbital period is defined by 


$$
P=\frac{2 \pi}{\dot{M}}
$$

where $\dot{M}$ is the mean rate-of-motion of the satellite across its orbit. This visibility value can be calculated for every point on the sphere, color-coded, and then plotted all together to produce a "visibility map" (see Fig. 19).

As mentioned in Section 3.4.1: Virtual Sphere Aligned with Satellite Orbit, second-order gravity effects will cause orbits to twist around relative to an Earthcentered inertial frame. (The effect of which will be discussed in greater detail in Section 3.5.2: Multiple-Day, Single-Satellite Coverage Estimation.) By definition, the orbit-fixed sphere will twist along with it. Therefore, if the visibility map for the orbit-fixed sphere can be computed once, then it can simply be reused for future times.

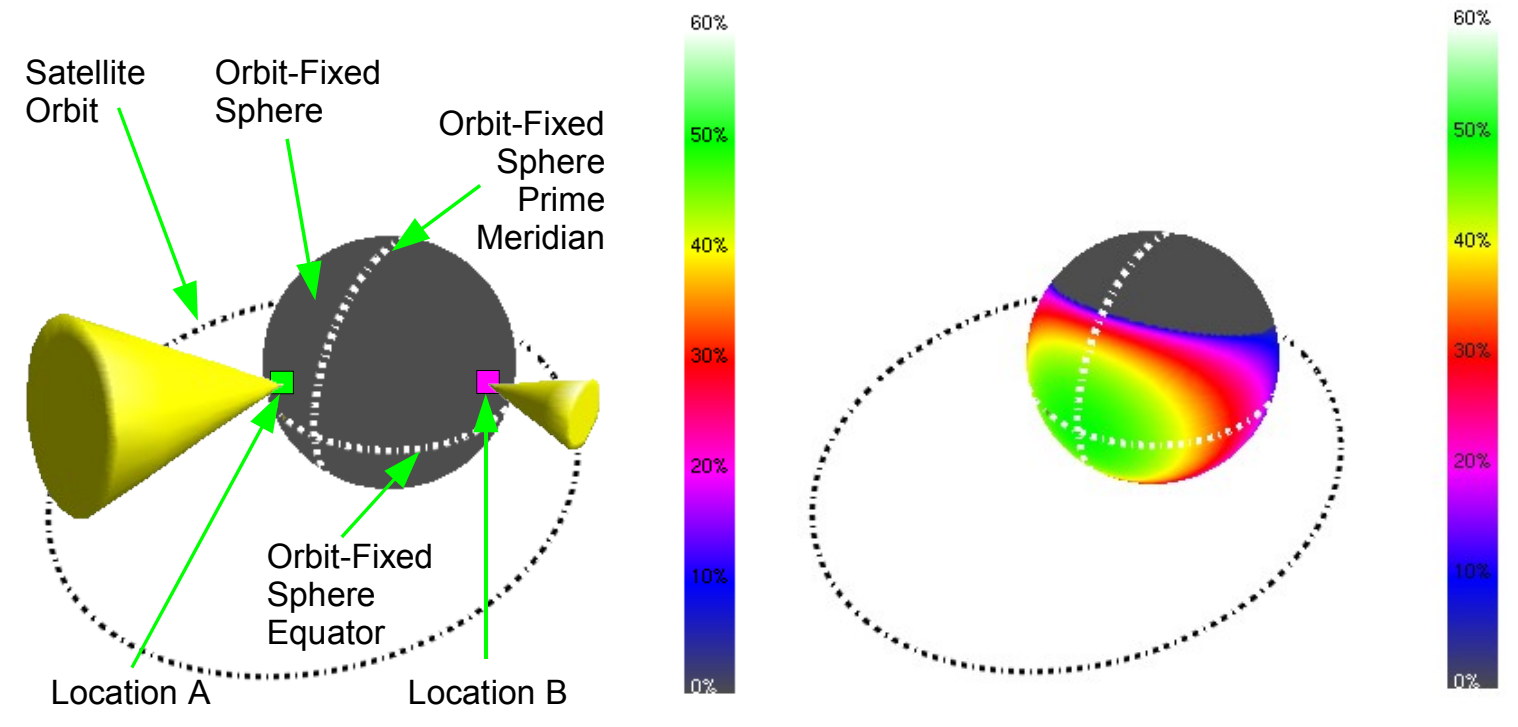

Figure 19: (Left) An LOS cone is projected outwards from two locations on the orbit-fixed sphere. For both locations, the fractional view of the orbital ellipse is calculated as in Eq. (23) and appropriately color-coded. (Right) For every point on the orbit-fixed sphere, the fractional view of the orbital ellipse is calculated as in Eq. (23) and appropriately colorcoded.

\subsection{3: Symmetry of Orbit-Fixed Sphere}

Fig. 20 shows how the orbit-fixed sphere is symmetric in two ways. It is symmetric across either side of the equator because the equator is co-planar with the orbital ellipse. Furthermore, since inspection of Fig. 7 clearly shows that the orbital ellipse is symmetric across its major axis, and since the orbit-fixed sphere's prime meridian is aligned with the orbital ellipse's major axis, the orbitfixed sphere is symmetric across the prime meridian.

Therefore, due to the symmetry of the orbit-fixed sphere, only one fourth of the orbit-fixed sphere needs to be computed. Referring to Fig. 20, assume that Quadrant A is computed. The data for any point in Quadrant B is the same as its mirror image, across the orbit-fixed sphere's equator, found in Quadrant A. The 
data for Quadrant D is similarly mirrored in Quadrant C. The data in Quadrant C is a "negative mirror" of the data across the orbit-fixed sphere's prime meridian found in Quadrant A. For example, if a data point in Quadrant A indicates that the view starts with a true anomaly of 35 degrees and finishes with a true anomaly of 110 degrees, then the mirror data point in Quadrant C will have a starting true anomaly of 250 degrees (-110 degrees after it has adjusted to a conventional representation between 0 and 360 degrees) and a finishing true anomaly of 325 degrees ( -35 degree after it has been adjusted to conventional representation).

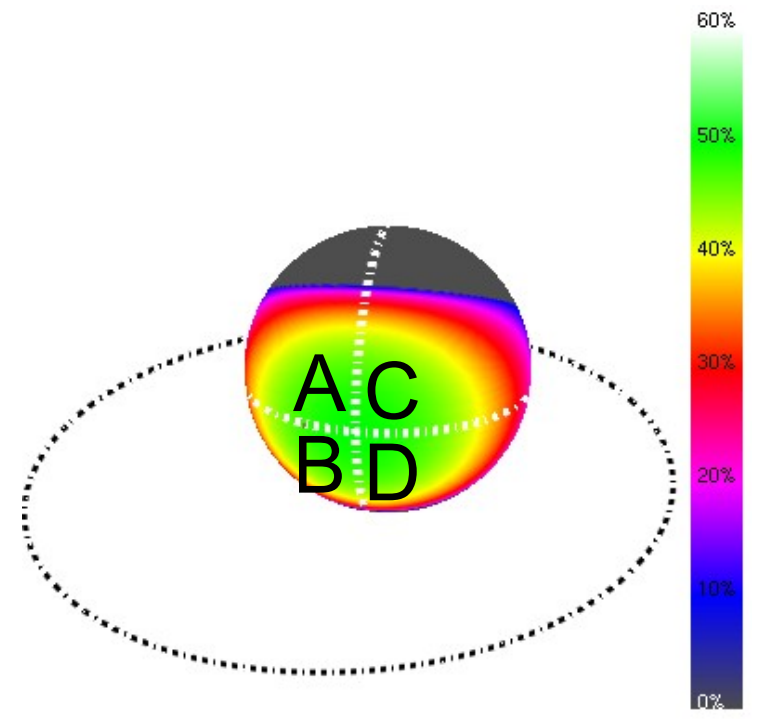

Figure 20: A satellite orbit and its corresponding orbit-fixed sphere. The orbit-fixed sphere is symmetric across its equator and its prime meridian.

\subsection{4: Earth Rotates Through a Virtual Inertial Sphere}

As discussed in Section 3.1: Classical Representation of an Orbit, each satellite orbit is classically represented relative to an inertial frame centered on the Earth. Since the orbit-fixed sphere is defined to be aligned with the orbital ellipse, it too can be represented relative to the inertial frame using the same parameters (e.g. $i, \Omega, \omega$ ) to describe its orientation/attitude.

Now imagine another virtual sphere that is also centered on the inertial frame, yet which is aligned with the inertial axes, and hence never rotates. This sphere will be called the "inertial sphere." Figure 21 depicts the relationships between satellite orbits, orbit-fixed spheres, and inertial spheres. The upper portion of the figure depicts how the orbit-fixed sphere and its visibility map rotate around with the overlying satellite orbit. The lower portion of the figure depicts the visibility map projected onto the inertial sphere before being unwrapped as a twodimensional surface. For each location on the inertial sphere, the orbital view can be determined as described in Section 3.2: Determining the View of a Satellite Orbit from a Point on a Sphere, or by extracting the orbital view from a 
pre-calculated, orbit-fixed sphere that overlays the inertial sphere.

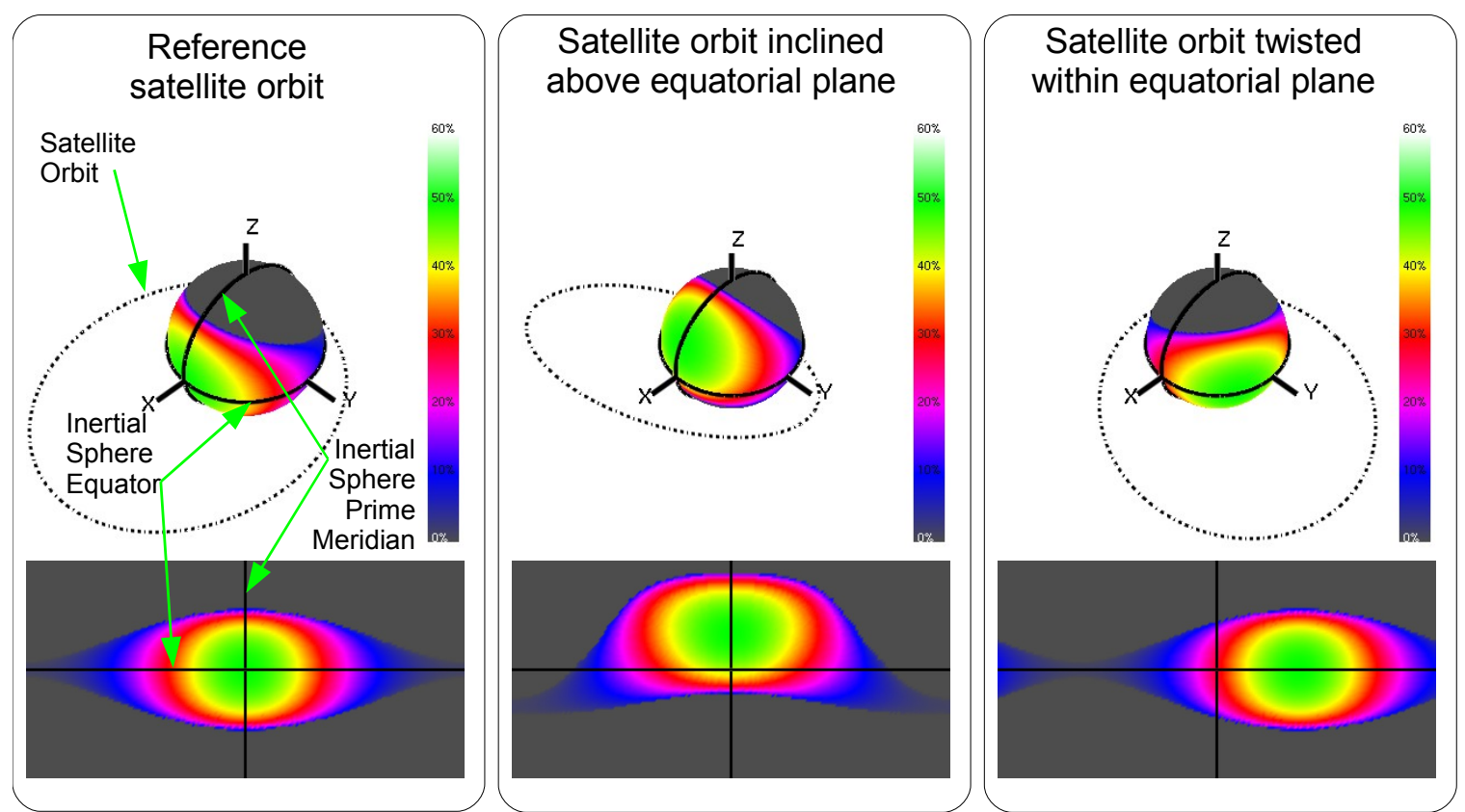

Figure 21: Three cases are shown. In each case, the upper portion depicts a satellite orbit and its visibility map on the underlying inertial sphere. The lower portion depicts the surface of the inertial sphere unwrapped as a 2D surface. Areas in gray are not visible/covered by a satellite. Areas in any other color are visible/covered from a satellite. The three cases depict a satellite orbit with the same semi-major axis and eccentricity but aligned differently relative to the inertial axes. Since the semi-major axis and eccentricity are the same, all three cases share the same orbit-fixed visibility map. However, since the orbit-fixed sphere maintains its alignment with the satellite orbit, the visibility map is realigned relative to inertial space, and hence, the inertial sphere.

The Earth also rotates relative to this inertial frame. Over the course of one planetary rotation in inertial space (i.e. sidereal day), any target on the Earth will travel across the latitude of the inertial sphere (see Fig. 22). As it traverses the inertial latitude, the target passes through different regions having larger and smaller views of the satellite orbit. This observation will be used to estimate the coverage for any given target. 

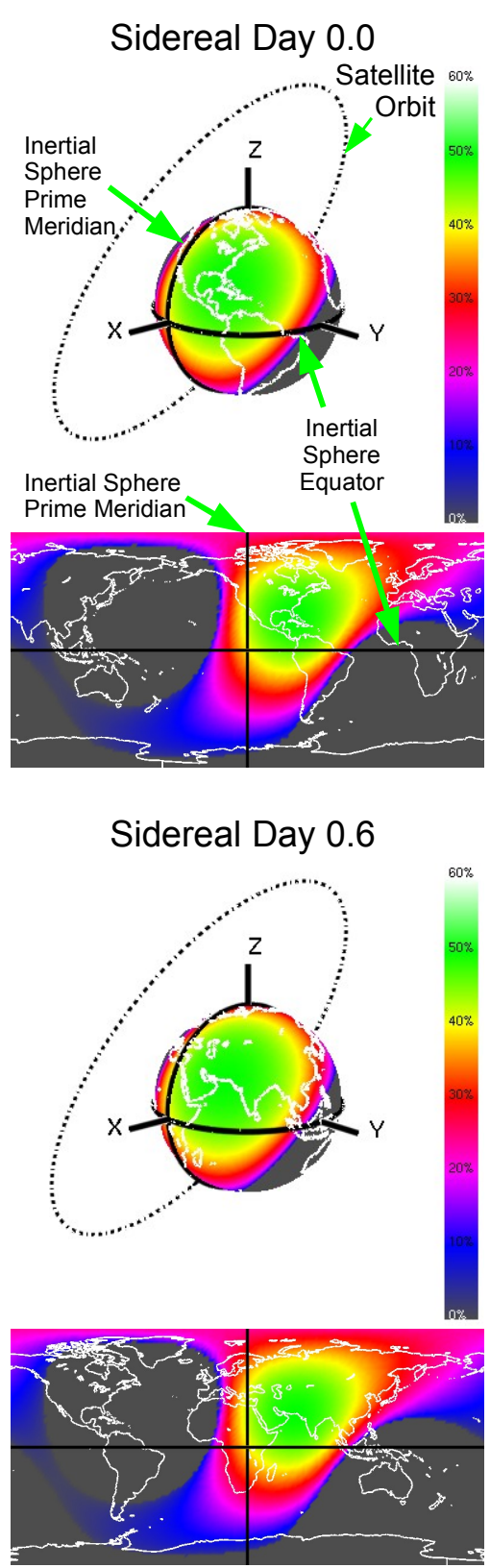

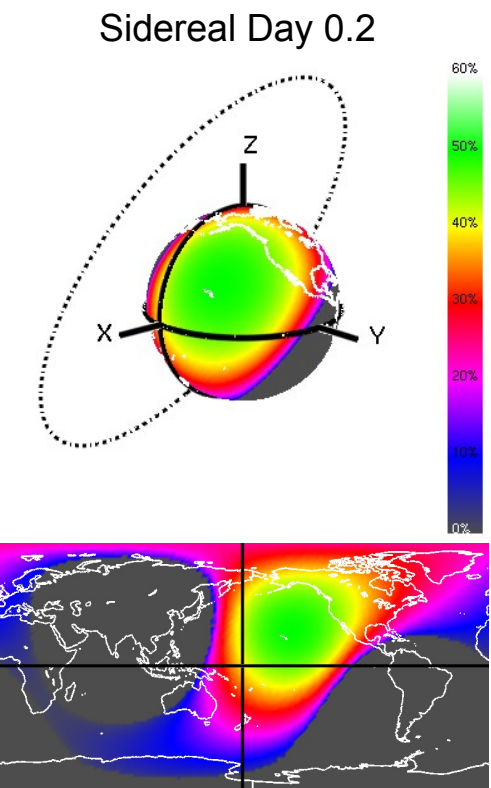

Sidereal Day 0.8
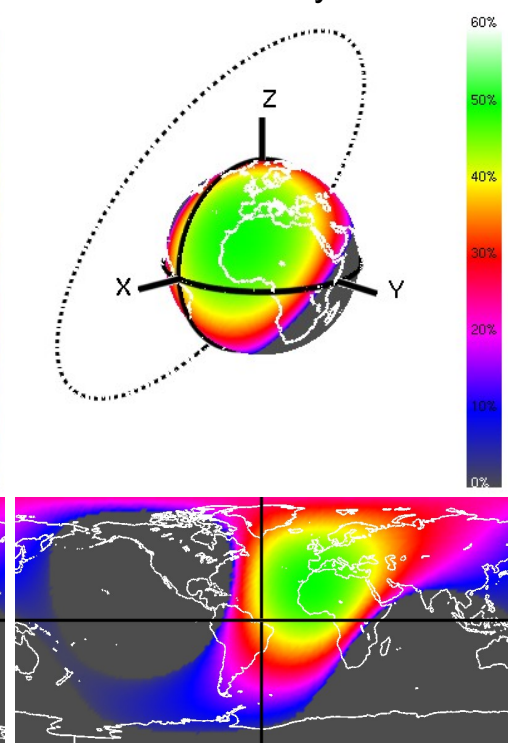

Sidereal Day 0.4
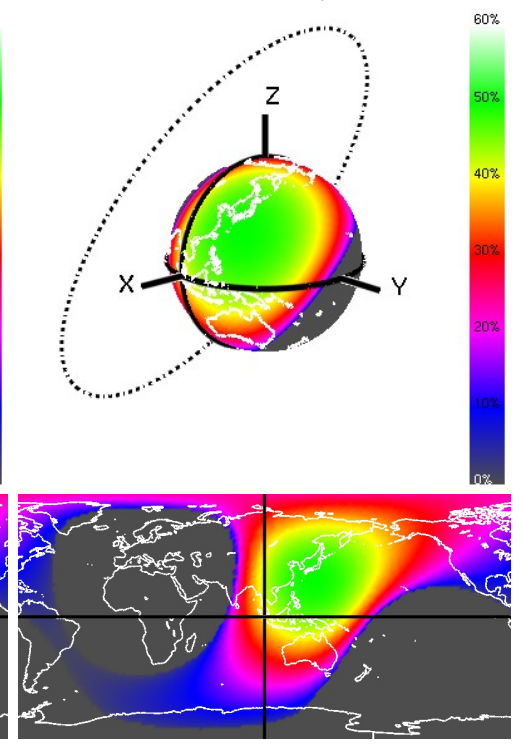

Sidereal Day 1.0
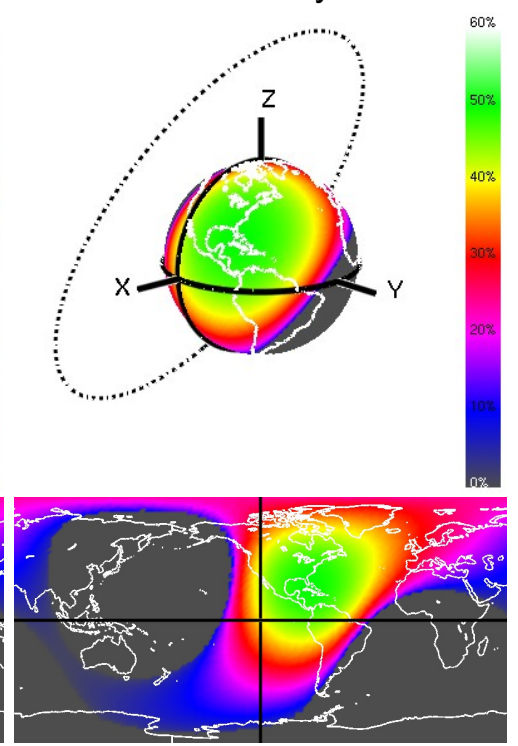

Figure 22: A 2D and 3D depiction of a satellite orbit and its visibility map over the course of a sidereal day. The satellite orbit and the Earth's surface are shown relative to a Cartesian, Earth-centered inertial (ECI) frame denoted by the X-Y-Z axes. The Earth's surface is identifiable by the outlines of the continents (white) on the 3D sphere and on the 2D representation of the inertial surface. By definition, the Earth's surface rotates once relative to the $\mathrm{ECl}$ frame during the sidereal day. The visibility map of the orbit is also shown on the 3D inertial sphere and on the 2D representation. Areas in gray are not visible/covered by a satellite. Areas in any other color are visible/covered from the satellite. 


\section{5: Estimating Coverage from Information across a Target's Latitude}

This section focuses on estimating coverage for a target by using the orbital view information from across the target's inertial latitude. Besides the assumptions made in the introduction to this chapter, this section further assumes that the satellite orbits have periods less than or equal to one-half of a sidereal day (i.e. approximately 11 hours 58 minutes). This assumption is true for $96 \%$ of non-geosynchronous Earth satellites (see Appendix A:Summary of Operational Satellites).

\subsection{1: Single-Day, Single-Satellite Coverage Estimation}

Coverage estimation is performed by identifying which portions of an orbit are in view as a target revolves in inertial space around the Earth's axis. In this model, the satellite's actual location, or mean anomaly (M), along its orbit is treated as unknown in order to estimate coverage solely from the geometry of the satellite orbit relative to the Earth. Different starting locations of the satellite will result in a range of coverage values with some being greater and some being less. Therefore, the approach taken here will be to provide upper and lower bounds in order to account for this modeling approach.

In this work, the visibility map that is projected onto the inertial sphere is computed once per sidereal day. Specifically, it is computed at the beginning of the sidereal day and then maintained constant as an approximation. This approximation is valid assuming that orbital geometry relative to the inertial frame does not change significantly over the course of a sidereal day (see Section 3.5.2: Multiple-Day, Single-Satellite Coverage Estimation). This approximation will be violated if a satellite maneuvers significantly. 

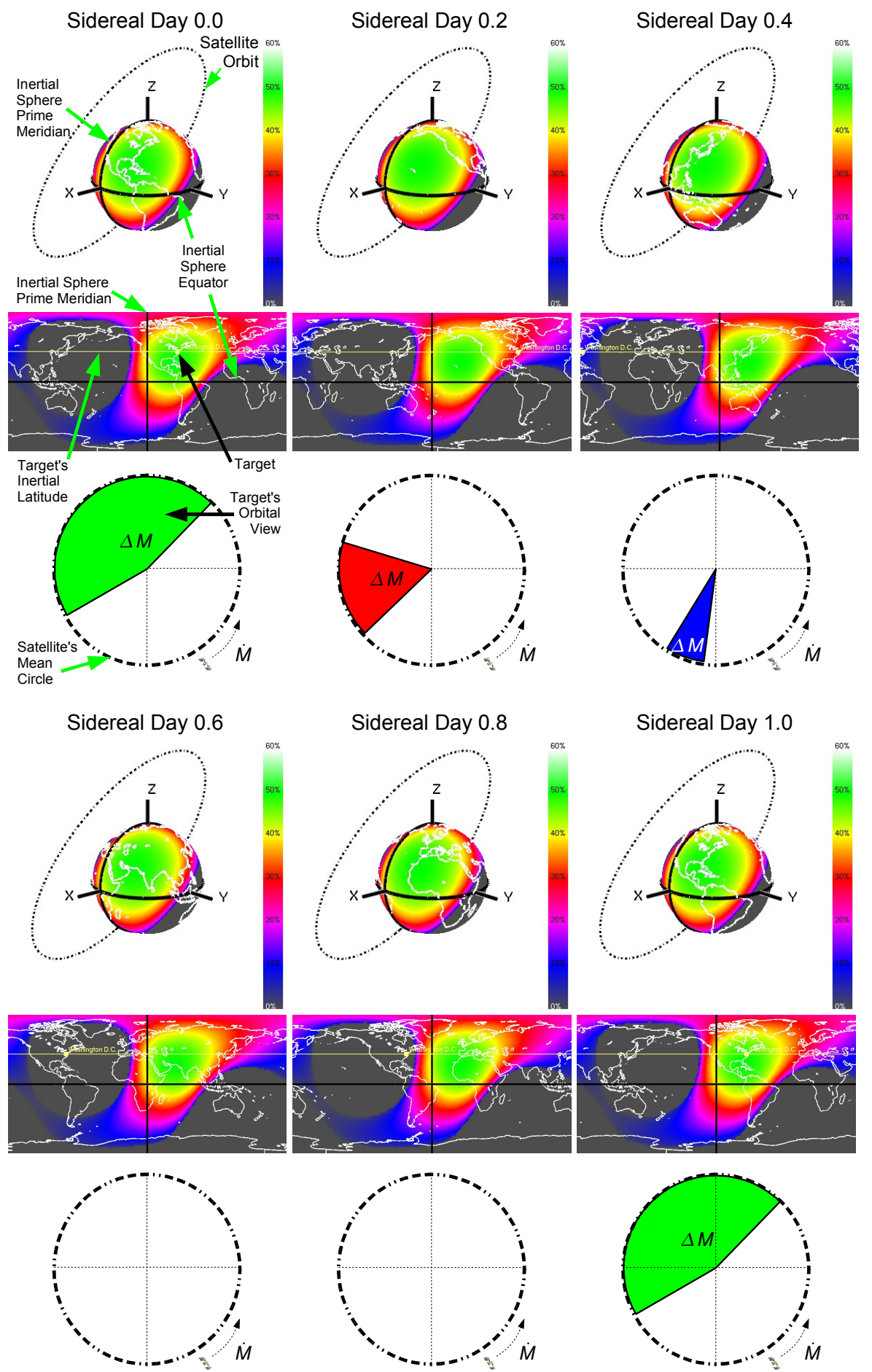

Figure 23: A depiction of how a target's orbital view changes over the course of a sidereal day. By definition, the Earth completes one revolution relative to an Earth-centered inertial-frame during a sidereal day. A target on the Earth's surface will rotate with it. A target's view of a satellite orbit will change during the sidereal day. At some points in time (i.e. inertial longitude) the satellite's orbit will be visible to one degree or another (e.g. Sidereal Day $0.0,0.2,0.4$, and 1.0). At other points in time, the satellite's orbit will not be visible (e.g. Sidereal Day 0.6 and 0.8 ). 
As the Earth rotates in inertial space once per sidereal day, so does a target move across an inertial latitude. A target's view of any satellite orbit changes during that transit (see Fig. 23). Invisible regions of a latitude are portions of the inertial latitude that have no visibility of a satellite orbit. Visible regions are portions of the inertial latitude that have some visibility of a satellite orbit.

Figure 24 qualitatively depicts the time-varying, orbital positions of the satellite, the target, and the target's orbital view. As a target moves across its inertial latitude (shown in blue), it travels through a region of visibility (delimited by the points $A$ and $B$ ) corresponding to where an LOS cone along that latitude intersects the orbit. The intersection on the satellite's mean circle is delimited by $M_{\llcorner B}$ and $M_{U B}$ (shown in green). It is only when the satellite is located between these points that it may be seen by the target. The exact timing of the coverage will depend on the phasing of the satellite relative to the target's view of the orbit.

It must be emphasized that merely viewing a portion of a satellite orbit does not connote that the satellite itself is visible. This is because the satellite may be situated/phased along another portion of the orbit at that instant in time. Only when the time-evolution of the target's orbital view is considered along with the satellite's motion across its orbit can some assertion on satellite visibility be made. 


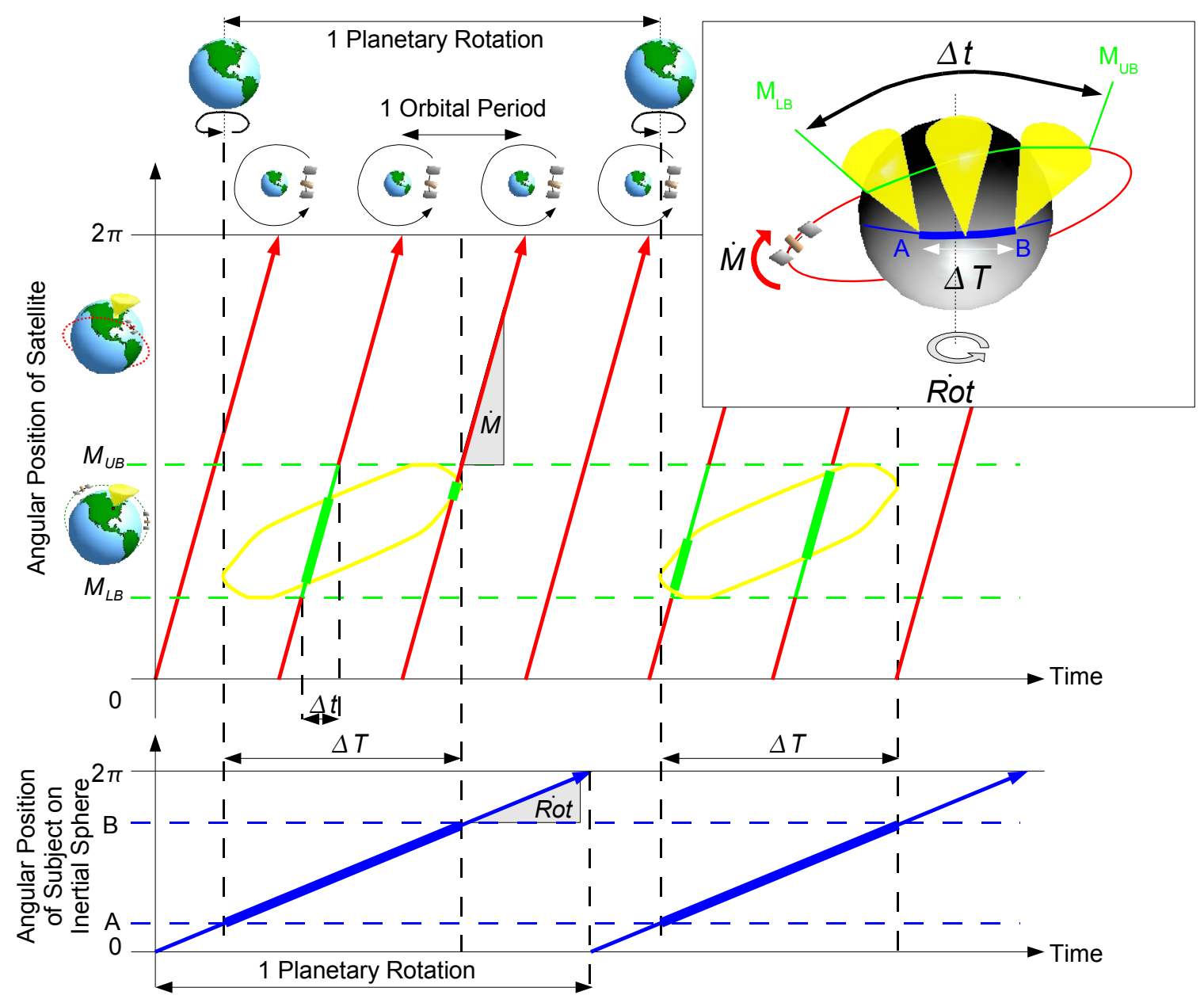

Figure 24: A qualitative depiction of how the visibility between a target on the Earth and an orbiting satellite is dependent on their relative timing/phasing. A target on the surface of the Earth moves across an inertial latitude as the Earth rotates (blue) at a rate of Rot. The satellite is in motion along its orbit (red and green) at a mean rate of $\dot{M}$. The points between $A$ and $B$ across the inertial latitude represent those points across the inertial latitude where line-of-sight cones (yellow) intersect the mean anomalies of $\mathbf{M}_{\mathrm{LB}}$ and $\mathbf{M}_{\mathrm{UB}}$ along the satellite orbit (green). The length of time required for the target to travel between points $A$ and $B$ is $\Delta T$. The length of time required for the satellite to rotate between points $\mathbf{M}_{\llcorner B}$ and $\mathbf{M}_{\cup B}$ is $\Delta t$. A target may only be seen by a satellite while the target passes between $A$ and $B$; even then, line-of-sight between the two is not attained until the satellite passes through the target's line-of-sight cone (yellow). The farther apart A and B are from each other, the greater the time that the target will be visible from the satellite orbit, thus increasing the number of viewing opportunities. 


\section{Numerically Estimating Coverage Across A Single, Visible, Latitude Region}

Coverage estimation is performed by simulating the motion of the satellite along its orbit while concurrently simulating the movement of the target's orbital view (see Fig. 24). The first step involves assuming an initial mean anomaly of the satellite along its orbit. The satellite's mean anomaly is then propagated in time at a constant rate of $\dot{M}$. Simultaneously, the orbital view of the mean circle is propagated through time. The satellite's position is then periodically checked to determine whether it falls within the orbital view which indicates visibility ${ }^{F}$. This is continued for the amount of time required for the target to traverse the visible region, $\Delta T$. The duration of each visible window is recorded as is the duration of each coverage gap.

This coverage estimation, however, is only appropriate for the presumed, initial mean anomaly. As a reminder to the reader, in this model, the satellite's actual mean anomaly is treated as unknown (in order to estimate coverage solely from the geometry of the satellite orbit relative to the Earth) and can vary anywhere between 0 and $2 \pi$. The effect of estimating coverage from a different, initial mean anomaly is that the satellite's position is shifted in time (see Fig. 25). The satellite will traverse a target's orbital view at different times thereby resulting in different coverage. ${ }^{G}$

Therefore, the numerical approach taken in this work is to enumerate all of the visibility windows and gaps that can possibly be encountered from different initial mean anomalies. A list of initial mean anomalies are enumerated between 0 (inclusive) and $2 \pi$ (exclusive) with angular separations, $\Delta M$, specified by

$$
\Delta M=\dot{M} \Delta t_{M A},
$$

where $\dot{M}$ is the mean orbital rate, and $\Delta t_{M A}$ is the time separation between any two starting anomalies. For each initial mean anomaly, the coverage is then determined over the course of one sidereal day by using the mean circle estimation described above.

F This coverage estimation approach is similar to the explicit approach taken in Section 2.3: Determining Visibility between Satellites and Targets but with the benefit of: simpler satellite dynamics (i.e. satellite motion across the mean circle at a constant rate of $\dot{M}$ ); pre-computed, static, orbital views from the orbit-fixed sphere; and simple time-evolution of the orbital views across a visible latitude region.

G This effect is also evident in Fig. 24, which shows the coverage over the course of two rotations of the Earth. By the start of the second rotation, the satellite's phase has shifted, and therefore, will be observed by the target at different positions along its inertial latitude (i.e. time). 

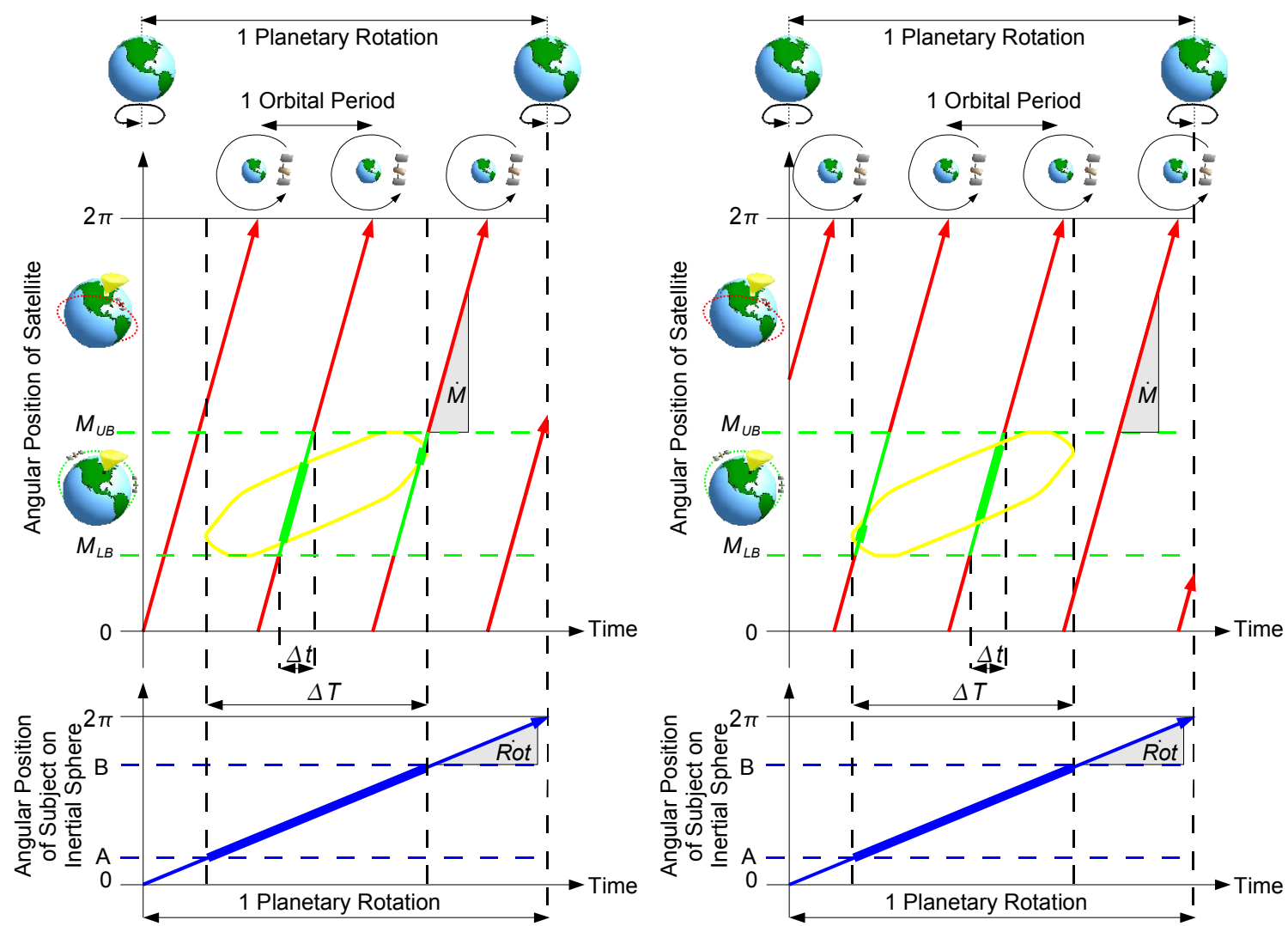

Figure 25: This variation on Fig. 24 depicts the effect that a satellite's initial mean anomaly has on the visibility between the satellite and a target on the Earth. The two plots differ because of the different initial position of the satellite. Therefore, each satellite's position (red) is shifted in time, thereby traversing the target's view of the satellite orbit (yellow) at different times.

\section{Numerically Aggregating Coverage Estimates From Multiple Latitude Regions}

Figure 23 depicts how any latitude on the inertial sphere can be decomposed into visible and invisible regions. Estimates of the coverage can be made for a target as it passes through each of the latitude regions. The results from each latitude region can be aggregated to arrive at a numerical coverage estimate for one sidereal day.

The coverage windows/visibility for each region can be estimated independently. The visible coverage for an entire sidereal day can then be determined by aggregating the coverage estimates from each visible region across the latitude.

Estimating the gaps/invisibility, however, is more complicated. Obviously, no coverage is available within the invisible latitude regions. However, even within a visible region, a target can traverse the latitude for some time before having the satellite come into view (as represented by its LOS cone). Therefore, an upper bound on the gap can be estimated for each visible region. If no visibility can be assured across a visible region then the visible region should be treated as an 
invisible region for the purpose of calculating the upper bound gap. When considering the upper bound gap across a latitude, the results from adjacent regions must be aggregated carefully. The upper bound gap must be extracted from the visible regions sandwiching an invisible region, and added to the total gap of the invisible region (i.e. the amount of time required to traverse the invisible region).

By applying all these ideas together, an estimate of the coverage for a sidereal day can be determined. A numerical study of the single-day coverage estimation can be found in Appendix D: Numerical Study of Single-Satellite Coverage Estimation.

One drawback of the approach described in this section is that it fails to account for visible latitude regions from multiple satellite orbits that overlap each other. Until that is addressed, coverage estimates for multiple satellites cannot be made except to say that the results from a single satellite estimation provide the worst-case result (where worst is dependent on the type of coverage being assessed such as total time, gaps, windows, etc.).

\subsection{2: Multiple-Day, Single-Satellite Coverage Estimation}

The orientations of satellite orbits (and their orbit-fixed spheres) twist in inertial space due to 2 nd-order perturbation effects. In the case of Earth, a dominant 2 nd-order perturbation is caused by the $\mathrm{J}_{2}$ gravitational term. The $\mathrm{J}_{2}$ term corresponds to the degree $n=2$ and to the order $m=0$, which are found in Eqs. (2) and (3).

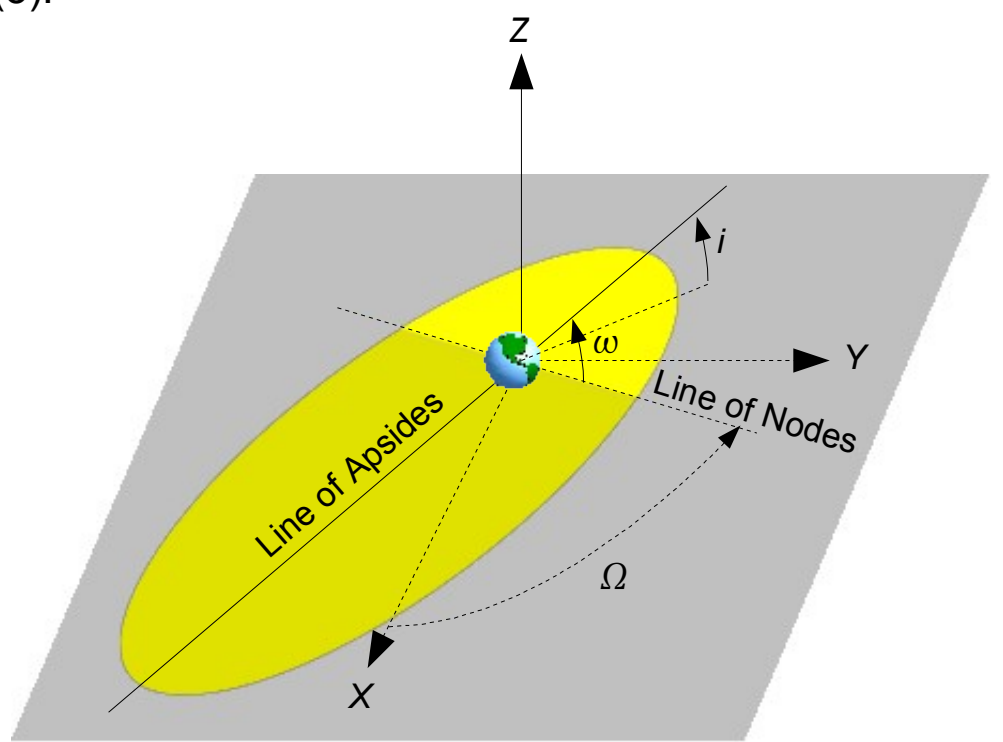

Figure 26: Depiction of the line of apsides and the line of nodes for a classical orbital ellipse.

Prussing and Conway 40 present an elegant derivation of how the $\mathrm{J}_{2}$ gravitational term affects the argument of periapsis (rotation of the apsides) and the right-ascension of the ascending node (regression of the nodes). The average rotation of the apsides is formulated as 


$$
\begin{aligned}
{\left[\frac{d \omega}{d t}\right]_{A V G} } & =\left[\frac{d \omega}{d \theta}\right]_{A V G}\left[\frac{d \theta}{d t}\right]_{A V G} \\
& =\left[\frac{d \omega}{d \theta}\right]_{A V G}[\dot{M}] \\
& =\left[\frac{3}{2} J_{2}\left\{\frac{R}{a\left(1-e^{2}\right)}\right\}^{2}\left\{2-\frac{5}{2} \sin ^{2}(i)\right\}\right][\dot{M}],
\end{aligned}
$$

where $R$ is the radius of the Earth. The geometrical effect of the rotation of the apsides is depicted in Fig. 27. A few observations regarding this effect are worth noting. First, note the dependence on both the eccentricity of the orbit, $e$, and its inclination, $i$. Second, this effect is meaningless for circular- or near-circular orbits since $\omega$ itself is ill-defined. Relatedly, the rotation of a circle within its plane of existence is also meaningless. Third, inspection of the $\left\{2-\frac{5}{2} \sin ^{2}(i)\right\}$ term within Eq. (24) reveals that two critical inclinations, at 63.4 degrees and 116.6 degrees, will cause the term to equal zero, and therefore, nullify any rotation of the apsides. The critical inclination at 63.4 degrees was used by the Soviet Union when it launched communication satellites into Molniya orbits; these were eccentric orbits with 12-hour periods whose apoapsides were maintained high over the northern latitudes of Earth.
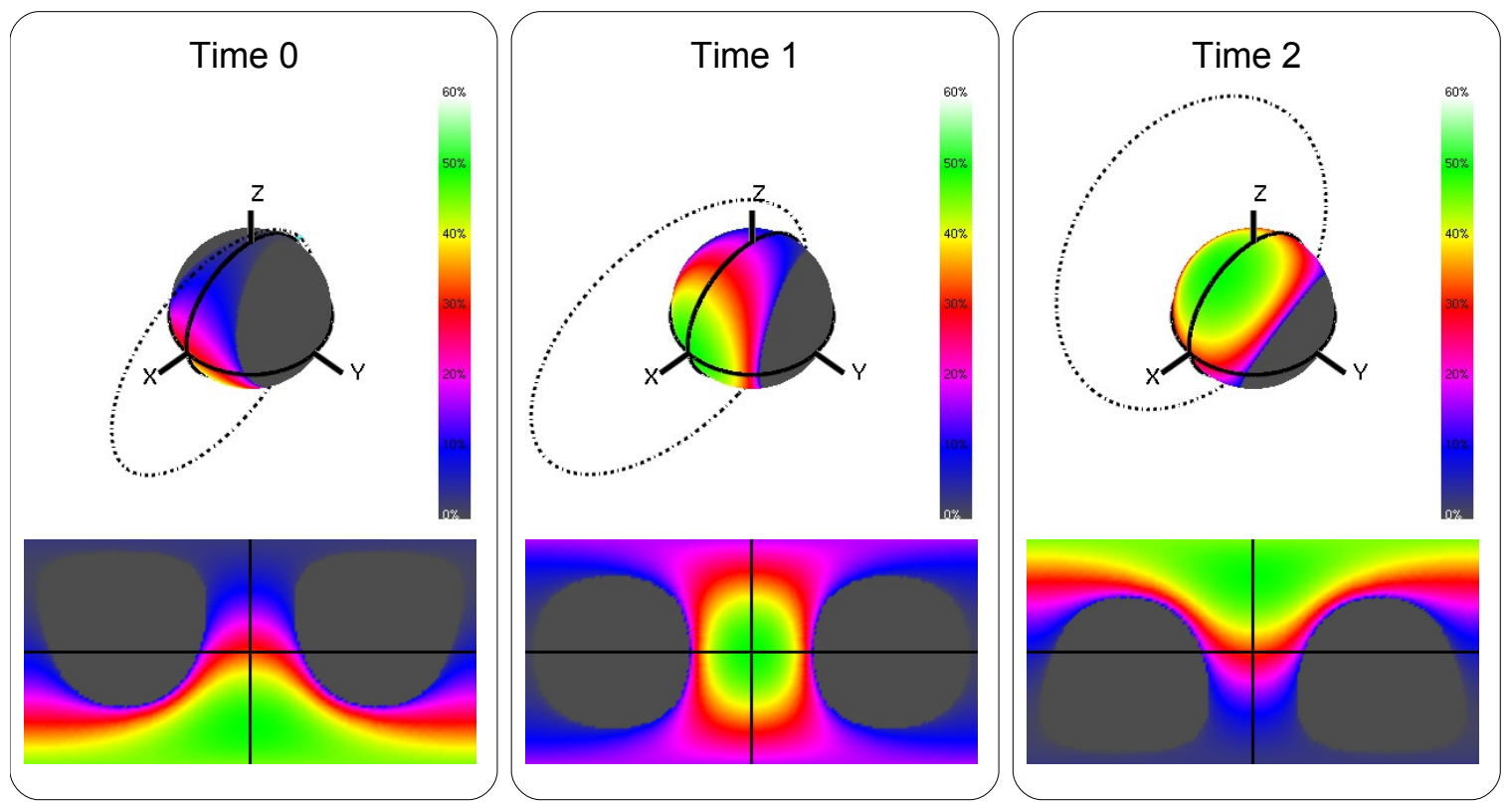

Figure 27: Depiction of the effect of rotation of the apsides on the satellite's orbit, and hence, its visibility map. Three points in time are shown. For each time, the upper portion depicts a satellite orbit and its visibility map on the underlying inertial sphere. The lower portion depicts the surface of the inertial sphere unwrapped as a two-dimensional surface. Areas in gray are not visible/covered by the satellite. Areas in any other color are visible/covered from the satellite. Note that this orbit is inclined to 90 degrees to nullify the effect of regression of the nodes. 
The average regression of the nodes is formulated as

$$
\begin{aligned}
{\left[\frac{d \Omega}{d t}\right]_{A V G} } & =\left[\frac{d \Omega}{d \theta}\right]_{A V G}\left[\frac{d \theta}{d t}\right]_{A V G} \\
& =\left[\frac{d \Omega}{d \theta}\right]_{A V G}[\dot{M}] \\
& =\left[\frac{-3}{2} J_{2}\left\{\frac{R}{a\left(1-e^{2}\right)}\right\}^{2} \cos (i)\right][\dot{M}],
\end{aligned}
$$

and its geometrical effect is depicted in Fig. 28. As for the rotation of the apsides, a couple of observations regarding this effect are worth pointing out. First, note the dependence on both the eccentricity of the orbit, $e$, and its inclination, $i$. Second, this effect is meaningless for equatorial- or nearequatorial orbits since $\Omega$ itself is ill-defined.
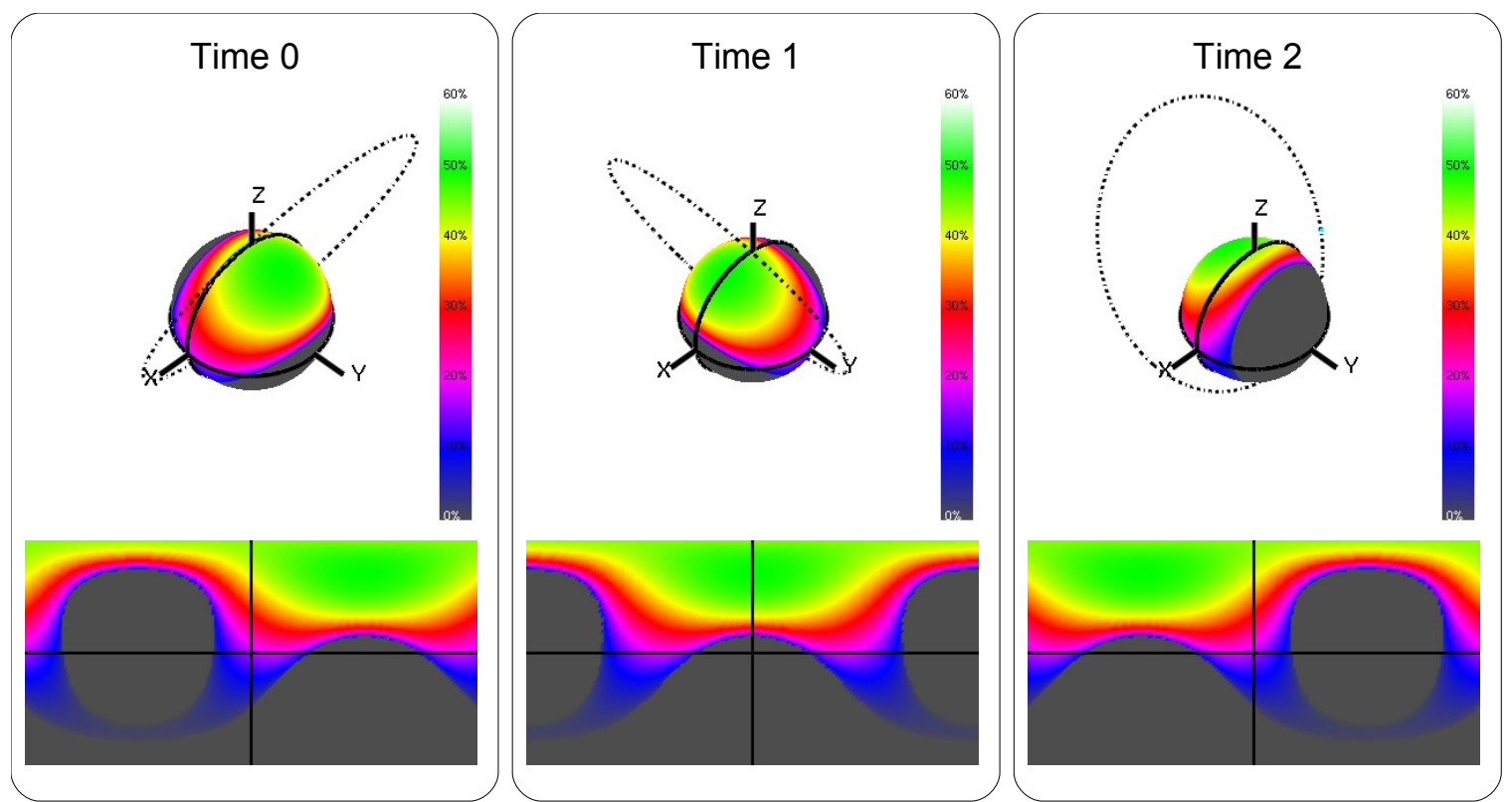

Figure 28: Depiction of the effect of regression of the nodes on the satellite's orbit, and hence, its visibility map. Three points in time are shown. For each time, the upper portion depicts a satellite orbit and its visibility map on the underlying inertial sphere. The lower portion depicts the surface of the inertial sphere unwrapped as a two-dimensional surface. Areas in gray are not visible/covered by the satellite. Areas in any other color are visible/covered from the satellite. Note that this orbit is inclined at one of two critical inclinations for Earth, 63.4 degrees, in order to nullify the effect of rotation of the apsides.

\section{Estimating Coverage Over Multiple Days}

In consideration of the effect of the $\mathrm{J}_{2}$ gravitational term on the orbit-fixed sphere's orientation, the following approach is taken in this work to approximate its effects. During the first planetary rotation (i.e. one sidereal day in the case of Earth) of coverage estimation, the perturbation effect will not have yet had any effect. At the beginning of each subsequent sidereal day, an instantaneous 
rotation of the apsides and an instantaneous regression of the nodes will be applied to the satellite orbit to account for the time during which the orbital ellipse was maintained fixed relative to inertial space. A numerical study of the multipleday coverage estimation can be found in Appendix D: Numerical Study of SingleSatellite Coverage Estimation.

\section{6: Summary}

This chapter discussed the link between a satellite's orbital geometry and the coverage that it provides. The link was identified by inspecting the view of satellite orbit from the surface of Earth-centered, virtual spheres. The orbital views can be portrayed as color-coded, visibility maps on the surface of those spheres. The rotation of the Earth through/underneath these visibility maps reveals patterns of coverage provided by a satellite over the course of a sidereal day. Estimation of coverage is possible by portraying the geometrical view as time-varying segments along a satellite's mean circle. The motion of the satellite across its mean circle can be treated like the hands of an analog clock in order to arrive at upper and lower bounds on visibility of a target. The Numerical Study of Single-Satellite Coverage Estimation shows examples of the coverage estimation bounding the coverage calculated by the approach shown in Chapter 2: Calculating Coverage. 


\section{Chapter 4: Maneuvering Strategies for Maximal Changes in Orbital Geometry}

This work is interested in reconfiguring satellite constellations in order to improve coverage. One approach to this problem is to find the optimal constellation arrangement for a given set of targets and then transform the existing constellation into it; this is investigated in Ref. 27 . However, that approach assumes that the existing constellation has sufficient propulsive capability and propellant to execute the transformation within the timespan of interest. This work investigates the change in orbital geometry that is feasible from a satellite's initial orbit subject subject to its limited propulsive thrust and limited propellant.

The previous chapter showed the direct relationship between a satellite's orbital geometry and the coverage that it provides. This chapter investigates maneuvering strategies that perform maximal changes to orbital geometry. In the next chapter, these maneuvering strategies will become parameters that are optimized in order to improve constellation coverage.

\section{1: Rate-of-Change of Keplerian Elements}

Gauss derived equations describing how the Keplerian orbital elements (see Classical Representation of an Orbit) vary as a function of perturbations that are defined relative to a local-vertical, local-horizontal (LVLH) reference frame attached to an orbiting satellite. This LVLH frame rotates within inertial space as the satellite travels along its orbit (see Fig. 1). The three generic, perturbing accelerations are:

- $a_{R}$ is an acceleration in the radial/vertical direction $\hat{e}_{R}$ (see Fig. 1).

- $a_{C T}$ is an acceleration pointing in the cross-track direction, $\hat{e}_{C T}$ (see

Fig. 1); this direction is parallel to the normal of the orbital plane.

- $a_{\theta}$ is an acceleration in the tangential/horizontal direction, $\hat{e}_{\theta}$ (see Fig. 1); this direction is determined from the cross-product of the $\hat{e}_{C T}$ direction with the $\hat{e}_{R}$ direction 41 .

The variational equations are ${ }^{42}$ :

$$
\begin{aligned}
& \frac{d \Omega}{d t}=\frac{r \sin (\theta)}{h \sin (i)} a_{C T}, \\
& \frac{d i}{d t}=\frac{r \cos (\theta)}{h} a_{C T}, \\
& \frac{d \omega}{d t}=\frac{1}{h e}\left[-p \cos (v) a_{R}+(p+r) \sin (v) a_{\theta}\right]-\frac{r \sin (\theta) \cos (i)}{h \sin (i)} a_{C T},
\end{aligned}
$$




$$
\begin{aligned}
& \frac{d a}{d t}=\frac{2 a^{2}}{h}\left(e \sin (v) a_{R}+\frac{p}{r} a_{\theta}\right), \\
& \frac{d e}{d t}=\frac{1}{h}\left(p \sin (v) a_{R}+[p+r \cos (v)+r e] a_{\theta}\right), \text { and } \\
& \frac{d M}{d t}=n+\frac{b}{a h e}\left[(p \cos (v)-2 r e) a_{R}-(p-r) \sin (v) a_{\theta}\right],
\end{aligned}
$$

where

$$
\begin{aligned}
& h=n a b \\
& n=\sqrt{\frac{\mu}{a^{3}}} \\
& b^{2}=\left|a^{2}\left(1-e^{2}\right)\right|, \\
& p=\frac{b^{2}}{a}=a\left(1-e^{2}\right), \\
& r=\frac{p}{1+e \cos (v)}=\frac{a\left(1-e^{2}\right)}{1+e \cos (v)}, \text { and } \\
& \theta=\omega+v .
\end{aligned}
$$

The following sections discuss maximizing the rate-of-change of the various parameters as a function of radial, $a_{R}$, tangential, $a_{\theta}$, and cross-track, $a_{C T}$, thrusting employed by the satellite. The general approach for optimizing these rates-of-change are as follows:

1. Formulate the rate-of-change equation as a function of the satellite's control inputs $\left(a_{R}, a_{\theta}\right.$, and $\left.a_{C T}\right)$ and the independent variable, $v$.

2. Take the derivative with respect to the control input.

3. By the necessary condition for optimality, set the derivative equal to zero, and solve for an expression of the control input as a function of the independent variable, $v$.

4. Plug the control back into the rate-of-change equation.

5. Confirm that the solution found is a maximal solution. 


\section{2: Maximization of the Rate-of-Change of the Right-Ascension of the Ascending Node}

This section seeks to find an orbital maneuver that maximizes the rate-ofchange of the right-ascension of the ascending node. Gauss' equation for the rate-of-change of the right-ascension of the ascending node (RAAN) is

$$
\frac{d \Omega}{d t}=\frac{r \sin (\theta)}{h \sin (i)} a_{C T},
$$

which is equivalent to

$$
\frac{d \Omega}{d t}=\frac{1-e^{2}}{n b \sin (i)} \frac{\sin (\omega+v)}{1+e \cos (v)} a_{C T} .
$$

Note that $1+e \cdot \cos (v)$ will always be positive for "closed" orbits $(e<1)$, which are the only types of orbits of interest in this work. Therefore, to maximize $\frac{d \Omega}{d t}$, $a_{C T}$ should have the same sign as $\sin (\omega+v)$. Letting

$$
a_{C T}=\max \operatorname{sign}(\sin (\omega+v)) \text {, }
$$

results in the following equation

$$
\frac{d \Omega}{d t}=\frac{1-e^{2}}{n b \sin (i)} \frac{\sin (\omega+v)}{1+e \cos (v)} \max \operatorname{sign}(\sin (\omega+v)) .
$$

Consider an auxiliary function for $\frac{d \Omega}{d t}$ that only considers those portions of the function varying with $v$,

$$
\frac{d \Omega_{\text {aux }}}{d t}=\frac{\sin (\omega+v)}{1+e \cos (v)} \operatorname{sign}(\sin (\omega+v)) .
$$

A necessary condition for this function to be maximized with respect to the true anomaly, $v$, is that its derivative with respect to $v$ equals zero; therefore,

$$
\begin{aligned}
\frac{d}{d v} \frac{d \Omega_{\text {aux }}}{d t} & =\frac{(1+e \cos (v))}{(1+e \cos (v))^{2}}\left[\cos (\omega+v) S+\sin (\omega+v) \frac{d S}{d v}\right] \\
& +\frac{(1+e \cos (v))}{(1+e \cos (v))^{2}}[+\sin (\omega+v) \operatorname{sesin}(v)] \\
& =0,
\end{aligned}
$$

where

$$
\begin{aligned}
& S=\operatorname{sign}(\sin (\omega+v)) \text { and } \\
& \frac{d S}{d v}=\frac{d}{d v} \operatorname{sign}(\sin (\omega+v)) .
\end{aligned}
$$

Multiply the function by the denominator, $(1+e \cos (v))^{2}$, to get 


$$
(1+e \cos (v))\left[\cos (\omega+v) S+\sin (\omega+v) \frac{d S}{d v}\right]+[\sin (\omega+v) S e \sin (v)]=0
$$

$\frac{d S}{d v}$ equals zero everywhere except for two points, $\omega+\nu=0$ and $\omega+\nu=\pi$. Therefore, the scaling $\sin (\omega+v)$ term will be ignored resulting in

$$
\cos (\omega+v) S+e \cos (v) \cos (\omega+v) S+e \sin (\omega+v) \sin (v) S=0 .
$$

Dividing by $S$ results in

$$
\cos (\omega+v)+e \cos (v) \cos (\omega+v)+e \sin (\omega+v) \sin (v)=0 .
$$

Using the trigonometric addition formulas,

$$
\begin{aligned}
& \sin (a+b)=\cos (a) \sin (b)+\sin (a) \cos (b) \text { and } \\
& \cos (a+b)=\cos (a) \cos (b)-\sin (a) \sin (b),
\end{aligned}
$$

results in

$$
\begin{aligned}
& \cos (\omega+v)+e \cos (v) \cos (\omega) \cos (v)-e \cos (v) \sin (\omega) \sin (v) \\
& +e \sin (v) \cos (\omega) \sin (v)+e \sin (v) \cos (v) \sin (\omega) \\
& =0 .
\end{aligned}
$$

Using the trigonometric identity, Eq. (18), and canceling two terms results in

$$
\cos (\omega+v)+e \cos (\omega)=0 \text {. }
$$

Solving for $\omega+v$ results in

$$
\operatorname{acos}(-e \cos (\omega))=\omega+\nu=\theta,
$$

for which there are two solutions for $\theta$. The first solution will be in the range of 0 to $\pi$. The second solution will equal $2 \pi-\theta$. This results in the following solutions:

$$
\begin{aligned}
& \theta_{1}=\operatorname{acos}(-e \cos (\omega)), \\
& \theta_{2}=2 \pi-\theta_{1}, \\
& v_{1}=\theta_{1}-\omega=\operatorname{acos}(-e \cos (\omega))-\omega, \text { and } \\
& v_{2}=\theta_{2}-\omega=2 \pi-a \cos (-e \cos (\omega))-\omega .
\end{aligned}
$$

In order to identify which of the two solutions is the maximum, one approach would be to check the second derivative. A simpler approach is to simply plug the two solutions into the equation for $\frac{d \Omega_{\text {aux }}}{d t}$ and to use the solution that yields a higher rate of change. 
Fig. 29 illustrates how $\frac{d \Omega_{\text {aux }}}{d t}$ varies with $v$ for a given eccentricity and argument of periapsis. It is worth emphasizing that the maximizing true anomaly is a function of both the eccentricity and the argument of periapsis.

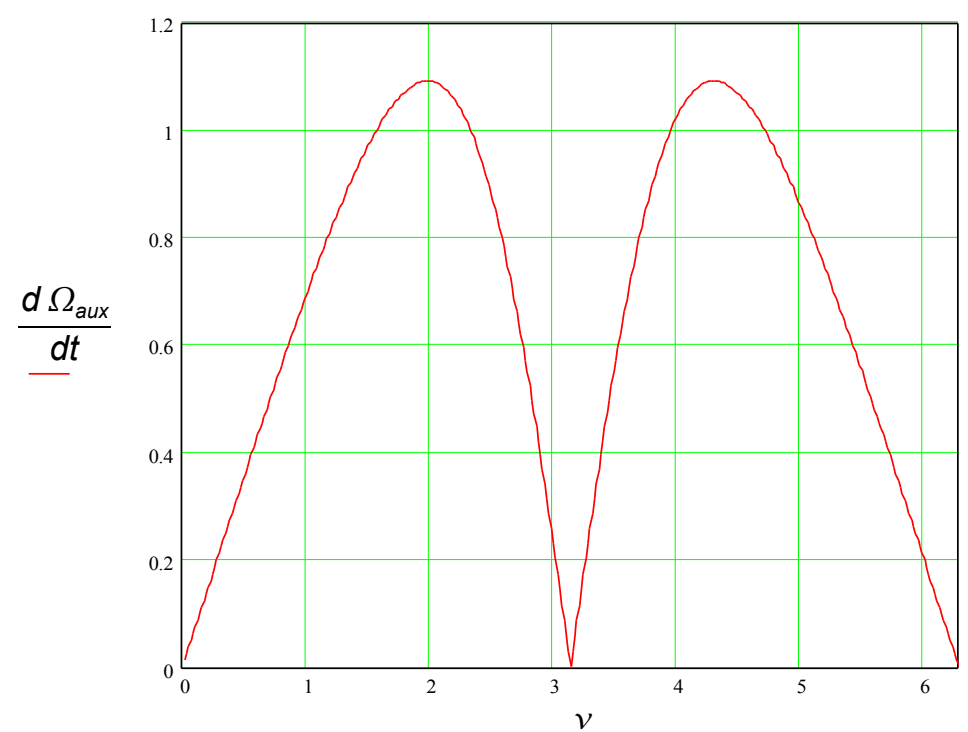

Figure 29 Auxiliary function for the rate of change of rightascension of the ascending nodes as a function of true anomaly [rad]. $(e=0.4, \omega=0)$

In summary, the control law described by Eq. (27) should be applied within a threshold-specified angular vicinity of whichever of the angles described by Eq. (29) maximizes $\frac{d \Omega_{a u x}}{d t}$. 


\section{3: Maximization of the Rate-of-Change of Inclination}

This section seeks to find an orbital maneuver that maximizes the rate-ofchange of the inclination. Gauss' equation for the rate-of-change of the semimajor axis is

$$
\frac{d i}{d t}=\frac{r \cos (\theta)}{h} a_{C T}
$$

which is equivalent to

$$
\frac{d i}{d t}=\frac{1-e^{2}}{n b} \frac{\cos (\omega+v)}{1+e \cos (v)} a_{C T} .
$$

Note that $1+e \cos (v)$ will always be positive for "closed" orbits $(e<1)$, which are the only types of orbits of interest in this work. Therefore, to maximize $\frac{d i}{d t}$, $a_{C T}$ should have the same sign as $\cos (\omega+\nu)$. Letting

$$
a_{C T}=\max \operatorname{sign}(\cos (\omega+v))
$$

results in the following equation

$$
\frac{d i}{d t}=\frac{1-e^{2}}{n b} \frac{\cos (\omega+v)}{1+e \cos (v)} \max \operatorname{sign}(\cos (\omega+v)) \text {. }
$$

Consider an auxiliary function for $\frac{d i}{d t}$ that only considers those portions of the function varying with $v$,

$$
\frac{d i_{a u x}}{d t}=\frac{\cos (\omega+v)}{1+e \cos (v)} \operatorname{sign}(\cos (\omega+v)) .
$$

A necessary condition for this function to be maximized with respect to the true anomaly, $v$, is that its derivative with respect to $v$ equals zero,

$$
\begin{aligned}
\frac{d}{d v} \frac{d i_{\text {aux }}}{d t} & =\frac{(1+e \cos (v))}{(1+e \cos (v))^{2}}\left[\cos (\omega+v) \frac{d Q}{d v}-Q \sin (\omega+v)\right] \\
& +\frac{(1+e \cos (v))}{(1+e \cos (v))^{2}}[+\cos (\omega+v) Q \cdot e \sin (v)] \\
& =0
\end{aligned}
$$

where

$$
\begin{aligned}
& Q=\operatorname{sign}(\cos (\omega+v)) \text { and } \\
& \frac{d Q}{d v}=\frac{d}{d v} \operatorname{sign}(\cos (\omega+v)) .
\end{aligned}
$$

Multiply the function by the denominator, $(1+e \cos (v))^{2}$, to get 


$$
(1+e \cos (v))\left[\cos (\omega+v) \frac{d Q}{d v}-Q \sin (\omega+v)\right]+[\cos (\omega+v) Q e \sin (v)]=0
$$

$\frac{d Q}{d v}$ equals zero everywhere except for two points, $\omega+\nu=\frac{\pi}{2}$ and $\omega+\nu=\frac{3 \pi}{2}$.

Therefore, the scaling $\cos (\omega+v)$ term will be ignored resulting in

$$
(1+e \cos (v))[-Q \sin (\omega+v)]+[\cos (\omega+v) Q e \sin (v)]=0 \text {. }
$$

Dividing by $Q$ results in

$$
(1+e \cos (v))[-\sin (\omega+v)]+[\cos (\omega+v) e \sin (v)]=0
$$

Expanding products and re-arranging results in

$$
e \sin (v) \cos (\omega+v)=\sin (\omega+v)+e \cos (v) \sin (\omega+v) \text {. }
$$

As in 4.2: Maximization of the Rate-of-Change of the Right-Ascension of the Ascending Node, using the trigonometric addition formulas, Eq. (28), and expanding results in

$$
\begin{aligned}
& e \sin (v) \cos (\omega) \cos (v)-e \sin (v)^{2} \sin (\omega) \\
& =\sin (\omega+v)+e \cos (v) \cos (\omega) \sin (v)+e \cos (v)^{2} \sin (\omega) .
\end{aligned}
$$

Using the trigonometric identity, Eq. (18), and canceling two term results in

$$
-e \sin (\omega)=\sin (\omega+v) \text {. }
$$

Solving for $\omega+v$ results in

$$
\operatorname{asin}(-e \sin (\omega))=\omega+v=\theta,
$$

for which there are two solutions for $\theta$. The first solution will be in the range of $\frac{-\pi}{2}$ to $\frac{\pi}{2}$. The second solution will equal $\pi-\theta$. This results in the following solutions:

$$
\begin{aligned}
& \theta_{1}=\operatorname{asin}(-e \sin (\omega)), \\
& \theta_{2}=\pi-\theta_{1}, \\
& v_{1}=\theta_{1}-\omega=\operatorname{asin}(-e \sin (\omega))-\omega, \text { and } \\
& v_{2}=\theta_{2}-\omega=\pi-\operatorname{asin}(-e \sin (\omega))-\omega .
\end{aligned}
$$

In order to identify which of the two solutions is the maximum, one approach would be to check the second derivative. A simpler approach is to simply plug the two solutions into the equation for $\frac{d i_{a u x}}{d t}$ and to use the solution that yields a higher rate of change. 
Fig. 30 illustrates how $\frac{d i_{\text {aux }}}{d t}$ varies with $v$ for a given eccentricity and argument of periapsis. It is worth emphasizing that the maximizing true anomaly is a function of both the eccentricity and the argument of periapsis.

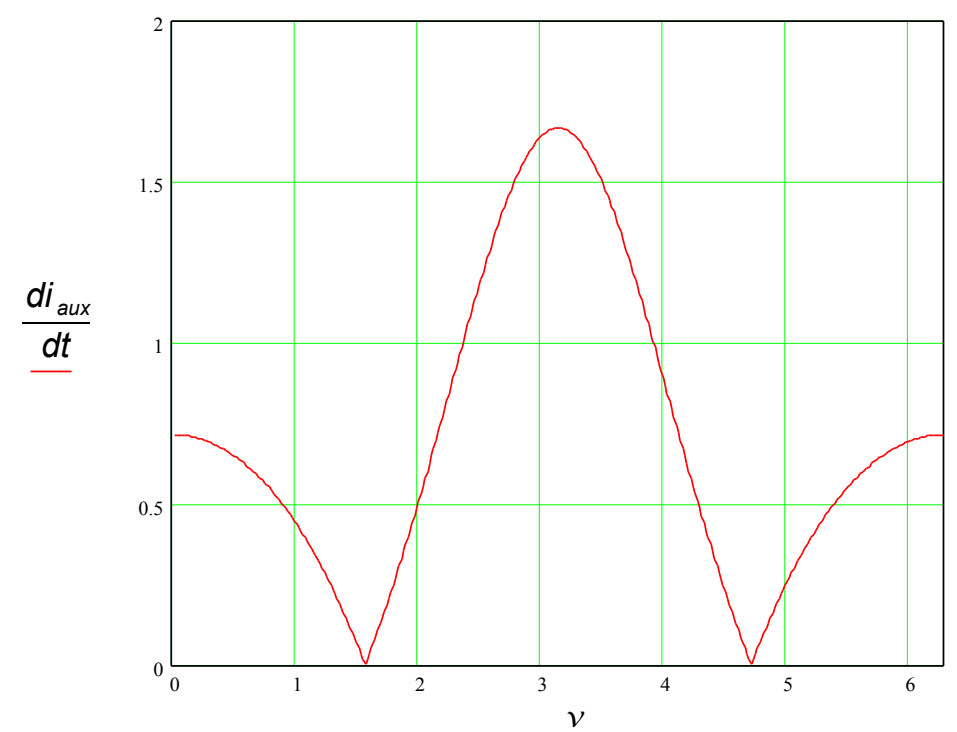

Figure 30 Auxiliary function for the rate of change of inclination as a function of true anomaly [rad]. $(e=0.4, \omega=0)$

In summary, the control law described by Eq. (31) should be applied within a threshold-specified angular vicinity of whichever of the angles described by Eq. (32) maximizes $\frac{d i_{a u x}}{d t}$.

\section{Special Condition: Initial Orbit is Circular and Posigrade-Equatorial}

By definition, posigrade-equatorial orbits have an inclination of zero degrees with an undefined RAAN, $\Omega$. Increasing an equatorial orbit's inclination is ambiguous unless the RAAN is specified. Inspection of Eqs. (30-31) reveals that an appropriately signed cross-track force, $a_{C T}$, will cause the inclination to suddenly become non-zero and therefore cause the RAAN to suddenly become defined at that location. The location of the desired RAAN (i.e. the ascending node) will be specified at a particular true longitude. A positive cross-track thrust will be applied within a user-specified, threshold of the desired ascending node, while a negative cross-track thrust will be applied within a user-specified, threshold of the desired descending node.

\section{Special Condition: Initial Orbit is Elliptical and Posigrade-Equatorial}

As previously stated, posigrade-equatorial orbits have an inclination of zero degrees with an undefined RAAN, $\Omega$. Inspection of Eqs. (30-31) reveals that an appropriately signed cross-track force, $a_{C T}$, will cause the inclination to suddenly become non-zero and therefore cause the RAAN to suddenly become defined at 
that location. The location of the desired RAAN (i.e. the ascending node) will be specified at a particular true longitude of periapsis. A cross-track thrust, $a_{C T}$, will be applied within a user-specified, threshold of either the desired ascending node or the desired descending node; the selection should be made by determining which of the two nodes results in a larger rate-of-change in the inclination, as determined by Eq. (31).

\section{Special Condition: Initial Orbit is Circular and Inclined}

If the initial orbit is circular then the periapsis and its associated argument of periapsis, $\omega$, is implicitly zero. Therefore, the optimal thrusting locations determined by Eq. (32) are near the ascending and descending nodes. The proximity of the satellite to these positions can be determined by checking the satellite's argument of latitude.

\section{Special Condition: Orbit Becoming Retrograde-Equatorial}

By definition, retrograde-equatorial orbits have an inclination of 180 degrees. When a satellite that is executing this maneuver causes the orbit's inclination to approach 180 degrees, the maneuvering will cease so as to not exceed this maximum inclination. (Exceeding this inclination would cause the inclination and the RAAN to switch by 180 degrees.) 


\section{4: Maximization of the Rate-of-Change of the Semi-Major Axis}

This section seeks to find an orbital maneuver that maximizes the rate-ofchange of the semi-major axis (SMA). Gauss' equation for the rate-of-change of the SMA is

$$
\frac{d a}{d t}=\frac{2 a^{2}}{h}\left(e \sin (v) a_{R}+\frac{p}{r} a_{\theta}\right)
$$

which is equivalent to

$$
\frac{d a}{d t}=\frac{2 a}{n b}\left(e \sin (v) a_{R}+(1+e \cos (v)) a_{\theta}\right) .
$$

It is assumed that the satellite has a single engine and can therefore only thrust/accelerate in a single direction. Therefore, $a_{R}$ will be defined as a function of $a_{\theta}$ by

$$
\begin{aligned}
& a_{R}=\sqrt{\max ^{2}-a_{\theta}^{2}} \operatorname{sign}(\sin (v))=\left[\max ^{2}-a_{\theta}^{2}\right]^{\frac{1}{2}} \operatorname{sign}(\sin (v)) \text { and } \\
& \frac{d a}{d t}=\frac{2 a^{2}}{n a b}\left(e \sin (v)\left[\max ^{2}-a_{\theta}^{2}\right]^{\frac{1}{2}} \operatorname{sign}(\sin (v))+(1+e \cos (v)) a_{\theta}\right) .
\end{aligned}
$$

Consider an auxiliary function for $\frac{d a}{d t}$ that only considers those portions of the function varying with $v$,

$$
\frac{d a_{a u x}}{d t}=\left(e \sin (v)\left[\max ^{2}-a_{\theta}^{2}\right]^{\frac{1}{2}} \operatorname{sign}(\sin (v))+(1+e \cos (v)) a_{\theta}\right) .
$$

A necessary condition for this function to be maximized with respect to the control input, $a_{\theta}$, is that its derivative with respect to $a_{\theta}$ equals zero; therefore,

$$
\frac{d}{d a_{\theta}} \frac{d a_{a u x}}{d t}=\left(e \sin (v)\left[\max ^{2}-a_{\theta}^{2}\right]^{\frac{-1}{2}} \operatorname{sign}(\sin (v))\left(-a_{\theta}\right)+(1+e \cos (v))\right)=0 \text {. }
$$

Multiplying the equation by $\left[\max ^{2}-a_{\theta}^{2}\right]^{\frac{1}{2}}$, and squaring the equation results in

$$
-(e \sin (v))^{2} a_{\theta}^{2}+(1+e \cos (v))^{2}\left[\max ^{2}-a_{\theta}^{2}\right]=0 .
$$

Solving for $a_{\theta}^{2}$ results in

$$
a_{\theta}^{2}=\frac{(1+e \cos (v))^{2} \max ^{2}}{(e \sin (v))^{2}+(1+e \cos (v))^{2}}=\frac{(1+e \cos (v))^{2} \max ^{2}}{e^{2}+2 e \cos (v)+1} .
$$

Inspection of Eq. (25d) reveals that to maximize the rate of change of the semimajor axis, the positive root is the desirable root of $a_{\theta}^{2}$, 


$$
a_{\theta}=\max \sqrt{\frac{(1+e \cos (v))^{2}}{e^{2}+2 e \cos (v)+1}} .
$$

Inserting the formula for $a_{\theta}$ into $a_{R}$ results in

$$
\begin{aligned}
a_{R} & =\left[\max ^{2}-\frac{(1+e \cos (v))^{2} \max ^{2}}{e^{2}+2 e \cos (v)+1}\right]^{\frac{1}{2}} \operatorname{sign}(\sin (v)) \\
& =\max \left[1-\frac{(1+e \cos (v))^{2}}{e^{2}+2 e \cos (v)+1}\right]^{\frac{1}{2}} \operatorname{sign}(\sin (v)) .
\end{aligned}
$$

Plugging these formulas for the control input back into the auxiliary function results in

$$
\begin{aligned}
\frac{d a_{a u x}}{d t} & =e \sin (v)\left[1-\frac{(1+e \cos (v))^{2}}{e^{2}+2 e \cos (v)+1}\right]^{\frac{1}{2}} \operatorname{sign}(\sin (v)) \\
& +(1+e \cos (v)) \sqrt{\frac{(1+e \cos (v))^{2}}{e^{2}+2 e \cos (v)+1}} .
\end{aligned}
$$

Fig. 31 shows an example of how $\frac{d a_{a u x}}{d t}$ varies with $v$ for a given eccentricity. The Figure illustrates that the greatest rate of change occurs when $v$ is near 0 (i.e. periapsis).

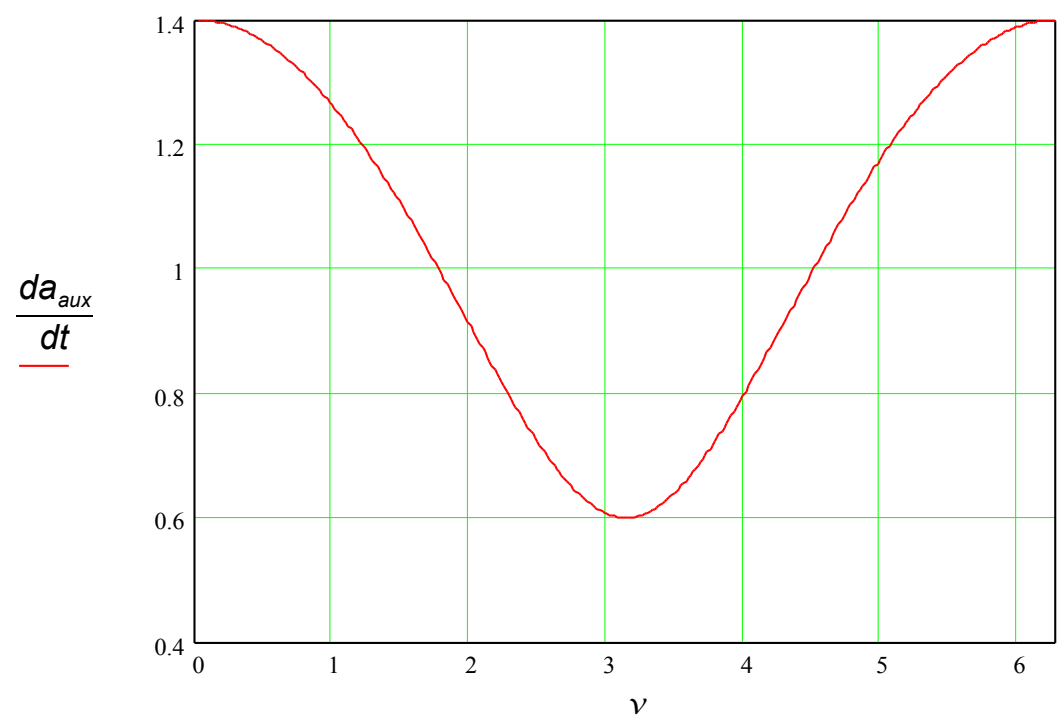

Figure 31 Auxiliary function for the rate of change of the semi-major axis as a function of true anomaly [rad]. $(e=0.4)$

Fig. 32 shows the corresponding optimal thrusts necessary to achieve the results shown in Fig. 31. 


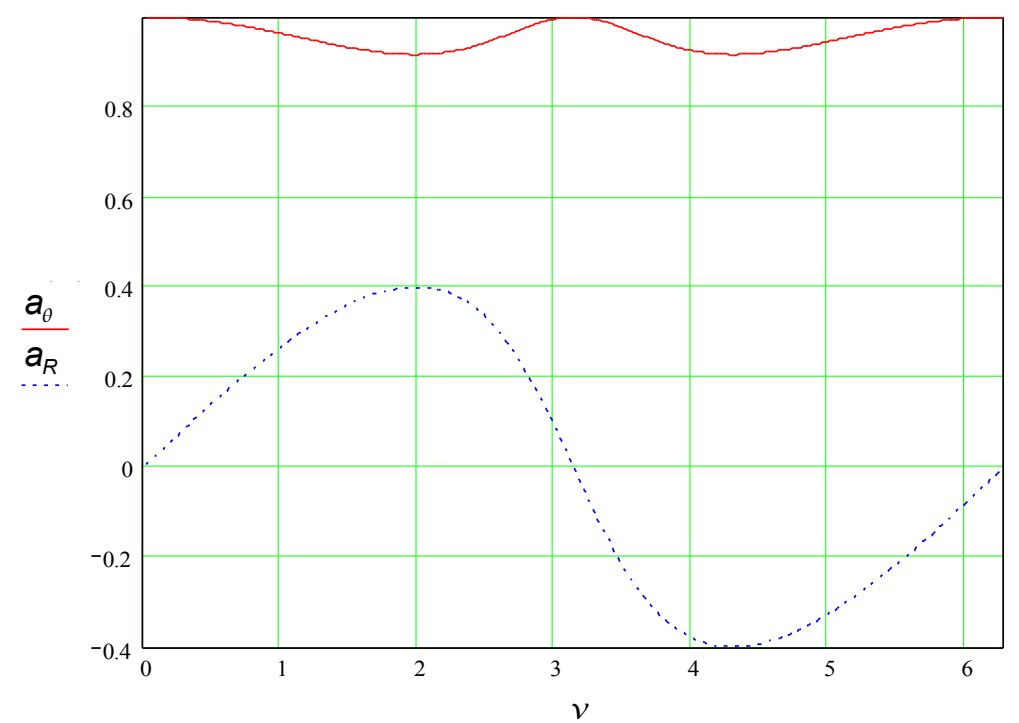

Figure 32 Normalized optimal thrust for maximizing the semimajor axis $(e=0.4)$

In summary, the control law described by Eq. (34) should be applied within a user-specified, threshold of the periapsis. Inspection of the control law applied within a user-specified, threshold of the periapsis reveals that it is equivalent to only using tangential thrust near periapsis.

\section{Special Condition: Initial Orbit is Circular and Equatorial}

If the initial orbit is circular, periapsis is not defined since all points in the orbit are equally close to the Earth. Since this maneuver is designed to increase the SMA, and since this maneuver is most effective when applied within a userspecified, threshold of the periapsis (i.e. a location on an eccentric orbit), it is assumed that driving the orbit into an eccentric orbit is desired. Therefore, there is now a choice in where the periapsis should be located. How this disambiguation angle is selected is left for a separate discussion in Section 5.2.3: Implementation of a Multiobjective Evolutionary Algorithm. The location of the periapsis will be specified at a particular true longitude. A tangential thrust will be applied within a user-specified, threshold of this location to transform the orbit into an eccentric orbit.

\section{Special Condition: Initial Orbit is Circular and Inclined}

In the case of a circular, inclined orbit, the location of the periapsis will be measured within the orbital plane from the line of the ascending node. The location of the periapsis will be specified at a particular argument of latitude. A tangential thrust will be applied within a user-specified, threshold of this location to transform the orbit into an eccentric orbit. 


\section{5: Maximization of the Rate-of-Change of Eccentricity}

This section seeks to find an orbital maneuver that maximizes the rate-ofchange of the eccentricity. Gauss' equation for the rate-of-change of the semimajor axis is

$$
\frac{d e}{d t}=\frac{1}{h}\left(p \sin (v) a_{R}+[(p+r) \cos (v)+r e] a_{\theta}\right),
$$

which is equivalent to

$$
\begin{aligned}
\frac{d e}{d t} & =\frac{1}{n a b}\left(a\left(1-e^{2}\right) \sin (v) a_{R}\right) \\
& +\frac{1}{n a b}\left(\left[\left(a\left(1-e^{2}\right)+\frac{a\left(1-e^{2}\right)}{1+e \cos (v)}\right) \cos (\theta)+\frac{a\left(1-e^{2}\right)}{1+e \cos (v)} e\right] a_{\theta}\right) .
\end{aligned}
$$

Collecting terms results in

$$
\frac{d e}{d t}=\frac{\left(1-e^{2}\right)}{n b}\left(\sin (v) a_{R}+\left[\left(1+\frac{1}{1+e \cos (v)}\right) \cos (v)+\frac{1}{1+e \cos (v)} e\right] a_{\theta}\right) \text {. }
$$

In order to maximize $\frac{d e}{d t}, a_{R}$ should have the same sign as $\sin (v)$. As in 4.4:

Maximization of the Rate-of-Change of the Semi-Major Axis, let

$$
a_{R}=\sqrt{\max ^{2}-a_{\theta}^{2}} \operatorname{sign}(\sin (v))=\left[\max ^{2}-a_{\theta}^{2}\right]^{\frac{1}{2}} \operatorname{sign}(\sin (v)),
$$

which leads to

$$
\begin{aligned}
\frac{d e}{d t} & =\frac{\left(1-e^{2}\right)}{n b}\left\{\sin (v)\left[\max ^{2}-a_{\theta}^{2}\right]^{\frac{1}{2}} \operatorname{sign}(\sin (v))\right\} \\
& +\frac{\left(1-e^{2}\right)}{n b}\left\{\left[\left(1+\frac{1}{1+e \cos (v)}\right) \cos (v)+\frac{1}{1+e \cos (v)} \cdot e\right] a_{\theta}\right\} .
\end{aligned}
$$

A necessary condition for this function to be maximized with respect to the control input, $a_{\theta}$, is that its derivative with respect to $a_{\theta}$ equals zero; thus,

$$
\begin{aligned}
\frac{d}{d a_{\theta}} \frac{d e}{d t} & =\frac{\left(1-e^{2}\right)}{n b}\left\{-\sin (v) \operatorname{sign}(\sin (v))\left(m a x^{2}-a_{\theta}^{2}\right)^{\frac{-1}{2}} a_{\theta}\right\} \\
& +\frac{\left(1-e^{2}\right)}{n b}\left[\left(1+\frac{1}{1+e \cos (v)}\right) \cos (v)+\frac{1}{1+e \cos (v)} e\right] \\
& =0 .
\end{aligned}
$$

The function equals zero either when the eccentricity, e, equals 1 and/or when the large term in brackets equals 0 . Since this work is only interested in cases 
where the eccentricity is less than 1 , it will only seek for where the large term equals 0 ; thus,

$$
\begin{aligned}
& -\sin (v) \operatorname{sign}(\sin (v))\left(\max ^{2}-a_{\theta}^{2}\right)^{\frac{-1}{2}} a_{\theta} \\
& +\left[\left(1+\frac{1}{1+e \cos (v)}\right) \cos (v)+\frac{1}{1+e \cos (v)} e\right] \\
& =0 .
\end{aligned}
$$

Letting $A=\left[\left(1+\frac{1}{1+e \cos (v)}\right) \cos (v)+\frac{1}{1+e \cos (v)} e\right]$ leads to

$$
\sin (v) \operatorname{sign}(\sin (v)) a_{\theta}=A\left(\max ^{2}-a_{\theta}^{2}\right)^{\frac{1}{2}} .
$$

Squaring both sides of the equation leads to

$$
\sin (v)^{2} a_{\theta}^{2}=A^{2}\left(\max ^{2}-a_{\theta}^{2}\right)=A^{2} \max ^{2}-A^{2} a_{\theta}^{2} .
$$

Solving for $a_{\theta}^{2}$ yields

$$
a_{\theta}^{2}=\frac{A^{2} \max ^{2}}{\sin ^{2}(v)+A^{2}} \text {. }
$$

Inspection of Eq. (25e) reveals that in order to maximize $\frac{d e}{d t}$, the sign of $a_{\theta}$ should be positive. Therefore, let

$$
a_{\theta}=\frac{A \max }{\sqrt{\left(\sin ^{2}(v)+A^{2}\right)}} \text {. }
$$

Plugging these formulas for the control input into Eq. (25e) results in

$$
\frac{d e}{d t}=\frac{\left(1-e^{2}\right)}{n b} \max \left(\sin (v)\left[1-\frac{A^{2}}{\sin ^{2}(v)+A^{2}}\right]^{\frac{1}{2}} \operatorname{sign}(\sin (v))+\frac{A^{2}}{\sqrt{\left(\sin ^{2}(v)+A^{2}\right)}}\right) .
$$

Consider an auxiliary function for $\frac{d e}{d t}$ that only considers those portions of the function varying with $v$,

$$
\frac{d e_{a u x}}{d t}=\sin (v)\left[1-\frac{A^{2}}{\sin ^{2}(v)+A^{2}}\right]^{\frac{1}{2}} \operatorname{sign}(\sin (v))+\frac{A^{2}}{\sqrt{\left(\sin ^{2}(v)+A^{2}\right)}} .
$$

Fig. 33 shows an example of how $\frac{d e_{a u x}}{d t}$ varies with $v$ for a given eccentricity. The figure illustrates that the greatest rate of change occurs when $v$ is near 0 (i.e periapsis) and near $\pi$ (i.e apoapsis). 


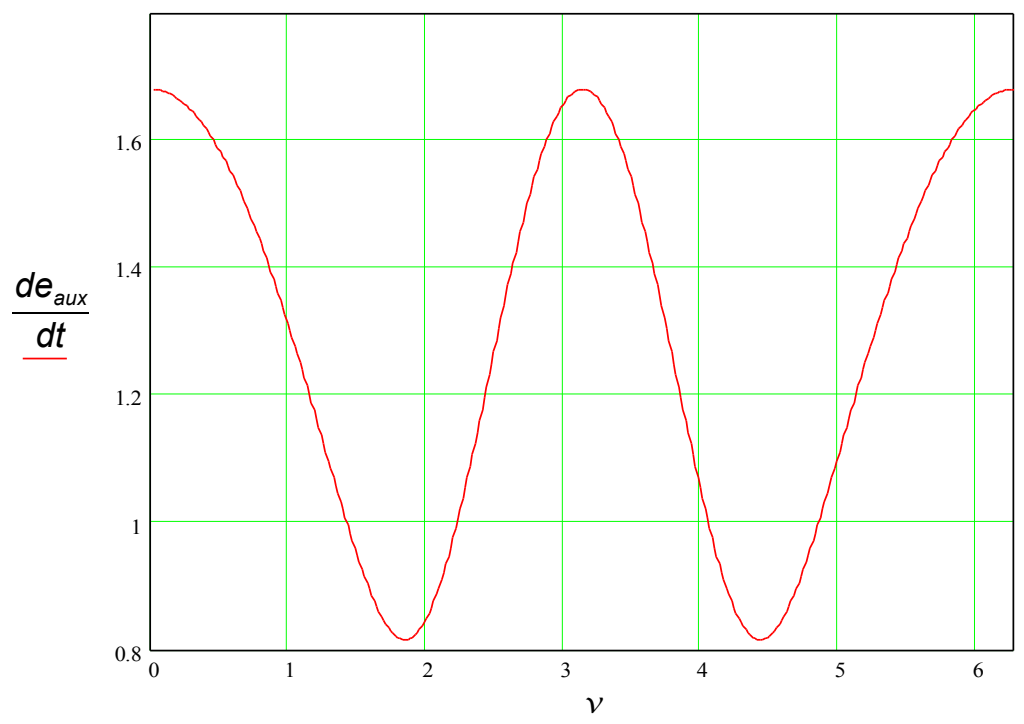

Figure 33 Auxiliary function for the rate of change of eccentricity as a function of true anomaly [rad]. $(e=0.4)$

Fig. 34 shows the corresponding optimal thrusts necessary to achieve the results shown in Fig. 33.

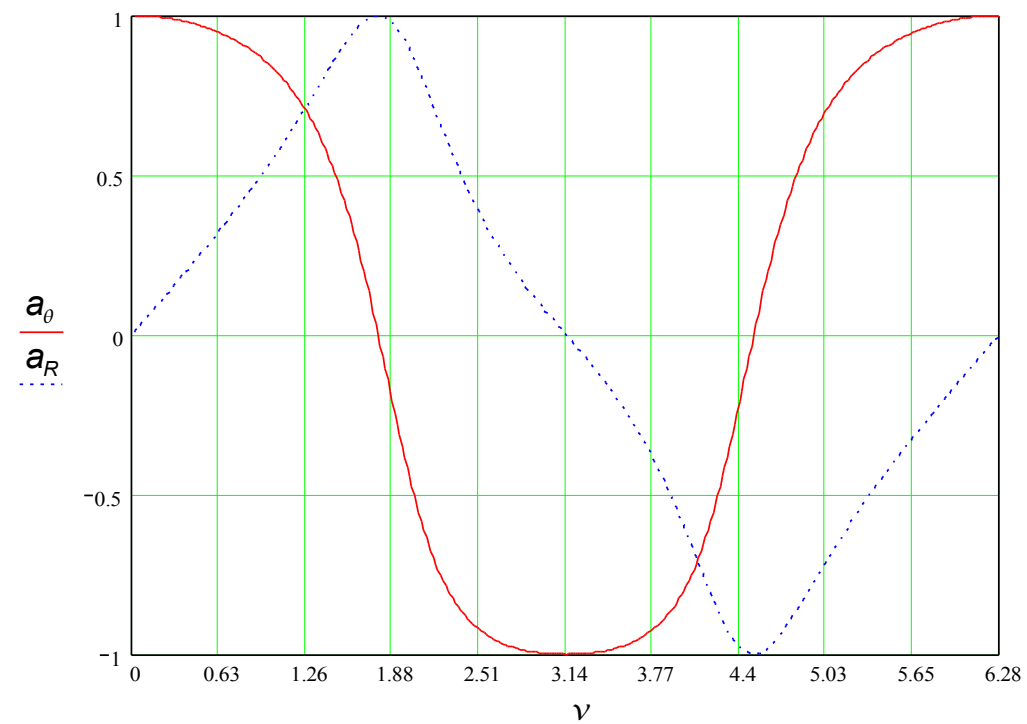

Figure 34 Normalized optimal thrust for maximizing the eccentricity $(e=0.4)$

In summary, the control law described by Eq. (36) should be applied within a user-specified, threshold of the periapsis and apoapsis. Inspection of the control law applied within a user-specified, threshold of the periapsis and near apoapsis reveals that is respectively equivalent to a positive tangential thrust and a negative tangential thrust. 


\section{Special Condition: Initial Orbit is Circular and Equatorial}

If the initial orbit is circular, periapsis is not defined since all points in the orbit are equally close to the Earth. Since this maneuver is designed to increase the eccentricity, and since this maneuver is most effective when applied within a user-specified, threshold of the periapsis and apoapsis (i.e. two locations on an eccentric orbit), it is assumed that driving the orbit into an eccentric orbit is desired. Therefore, there is now a choice in where the periapsis should be located. How this disambiguation angle is selected is a separate discussion in Section 5.2.3: Implementation of a Multiobjective Evolutionary Algorithm. The location of the periapsis will be specified at a particular true longitude. A tangential thrust will be applied within a user-specified, threshold of the this location to transform the orbit into an eccentric orbit.

\section{Special Condition: Initial Orbit is Circular and Inclined}

In the case of a circular, inclined orbit, the location of the periapsis will be measured within the orbital plane from the line of the ascending node. The location of the periapsis will be specified at a particular argument of latitude. A tangential thrust will be applied within a user-specified, threshold of this location to transform the orbit into an eccentric orbit.

\section{Caution when Thrusting at Apoapsis}

As stated in the conclusion, a negative tangential thrust will be applied within a user-specified, threshold of the apoapsis in order to increase the eccentricity. Caution must be taken with this maneuver especially in light of its effect on the SMA. Recall,

$$
\frac{d a}{d t}=\frac{2 a}{n b}\left(e \sin (v) a_{R}+(1+e \cos (v)) a_{\theta}\right) .
$$

A negative tangential thrust at apoapsis, $v=\pi$, also has the effect of reducing the SMA. If the maneuver at apoapsis is carried out too long, then the eccentricity, $e$, will decrease and the SMA, $v$, will decrease. The periapsis is the point of closest approach to the Earth and is equal to (by an application of Eq. (5))

$$
r_{p}=a(1-e) .
$$

At some point, the maneuver at apoapsis has the potential to cause the periapsis to approach dangerously close to the Earth. Therefore, some minimum safety radius should be specified to prohibit the maneuver from endangering the satellite. 


\section{6: Maximization of the Rate-of-Change of Argument of Periapsis}

This section seeks to find an orbital maneuver that maximizes the rate-ofchange of argument of periapsis, $\omega$. Note that this maneuver is irrelevant when the initial orbit is circular $(e=0)$ due to the argument of periapsis not being defined for such orbits.

Gauss' equation for the rate-of-change of the argument of periapsis is

$$
\frac{d \omega}{d t}=\frac{1}{h e}\left[-p \cos (v) a_{R}+(p+r) \sin (v) a_{\theta}\right]-\frac{r \sin (\theta) \cos (i)}{h \sin (i)} a_{C T},
$$

which is equivalent to

$$
\begin{aligned}
\frac{d \omega}{d t} & =\frac{1-e^{2}}{e n b}\left[-\cos (v) a_{R}+\left(1+\frac{1}{1+e \cos (v)}\right) \sin (v) a_{\theta}\right] \\
& -\frac{1-e^{2}}{n b} \frac{\cos (i)}{\sin (i)} \frac{\sin (\omega+v)}{1+e \cos (v)} a_{C T} .
\end{aligned}
$$

This work will optimize the in-plane thrusts, $a_{R}$ and $a_{\theta}$, and the out-of-plane thrust, $a_{C T}$, separately.

\subsection{1: Out-of-plane thrust only}

Consider the out-of-plane thrust, $a_{C T}$. In order to maximize $\frac{d \omega}{d t}, a_{C T}$ should have the same sign as $-\cos (i) \sin (\omega+\nu)$ since the inclination, $\mathrm{i}$, is always between 0 and $\pi$. Letting

$$
T(v)=\operatorname{sign}(-\cos (i) \sin (\omega+v))
$$

and

$$
a_{C T}=\max T
$$

results in

$$
\frac{d \omega}{d t}=\frac{1-e^{2}}{e n b}-\frac{1-e^{2}}{n b} \frac{\cos (i)}{\sin (i)} \frac{\sin (\omega+v)}{1+e \cos (v)} \max T .
$$

Consider an auxiliary function for $\frac{d \omega}{d t}$ that only considers those portions of the function varying with $v$,

$$
\frac{d \omega_{a u x}}{d t}=\frac{\sin (\omega+v)}{1+e \cos (v)} T .
$$

A necessary condition for this function to be maximized with respect to the true anomaly, $v$, is that its derivative with respect to $v$ equals zero. 


$$
\begin{aligned}
\frac{d}{d v} \frac{d \omega_{\text {aux }}}{d t} & =\frac{(1+e \cos (v))\left(\sin (\omega+v) \frac{d T}{d v}+T \cos (\omega+v)\right)}{(1+e \cos (v))^{2}} \\
& +\frac{\sin (\omega+v) T e \sin (v)}{(1+e \cos (v))^{2}} \\
& =0
\end{aligned}
$$

Multiply the function by the denominator, $(1+e \cos (v))^{2}$, to get

$$
(1+e \cos (v))\left(\sin (\omega+v) \frac{d T}{d v}+T \cos (\omega+v)\right)+\sin (\omega+v) T e \sin (v)=0
$$

$\frac{d T}{d v}$ equals zero everywhere except for two points, $\omega+\nu=0$ and $\omega+\nu=\pi$. Therefore, the scaling $\sin (\omega+v)$ term will be ignored, so

$$
(1+e \cos (v))(T \cos (\omega+v))+\sin (\omega+v) T e \sin (v)=0 \text {. }
$$

Dividing by $T$ and expanding results in

$$
\cos (\omega+v)+e \cos (v) \cos (\omega+v)+\sin (\omega+v) e \sin (v)=0 \text {. }
$$

Using the trigonometric additional formulas, Eq. (28), results in

$$
\begin{aligned}
\cos (\omega+v) & +e \cos (v) \cos (\omega) \cos (v)-e \cos (v) \sin (\omega) \sin (v) \\
& +e \sin (v) \cos (\omega) \sin (v)+e \sin (v) \sin (\omega) \cos (v) \\
& =0 .
\end{aligned}
$$

Using the trigonometric identity, Eq. (18), and canceling two terms results in

$$
\cos (\omega+v)+e \cos (\omega)=0 \text {. }
$$

As in 4.2: Maximization of the Rate-of-Change of the Right-Ascension of the Ascending Node, the two roots of this equation are defined by

$$
\begin{aligned}
& \theta_{1}=\operatorname{acos}(-e \cos (\omega)), \\
& \theta_{2}=2 \pi-\theta_{1}, \\
& v_{1}=\theta_{1}-\omega=\operatorname{acos}(-e \cos (\omega))-\omega, \text { and } \\
& v_{2}=\theta_{2}-\omega=2 \pi-\operatorname{acos}(-e \cos (\omega))-\omega .
\end{aligned}
$$

In summary, the control law described by Eq. (38) should be applied within a user-specified, threshold of whichever of the angles described by Eq. (29) maximizes $\frac{d \omega_{a u x}}{d t}$.

However, it is worth noting that the effect of an out-of-plane thrusting on $\frac{d \omega}{d t}$ is further scaled by a factor that is not dependent on the true anomaly, 


$$
\frac{1-e^{2}}{n b} \frac{\cos (i)}{\sin (i)}
$$

This scaling factor is zero for polar orbits $\left(i=\frac{\pi}{2}\right)$, and approaches infinity for orbits approaching the equatorial plane $(i=0$ and $i=\pi)$. This infinite rate-ofchange is closely associated to the rate-of-change of the RAAN, $\Omega$, which is proportional to the same out-of-plane thrusting and which is not-defined for equatorial orbits. In reality, an orbit that is retrograde and nearly equatorial $(i=\pi-\epsilon)$, and whose inclination is increased beyond $\pi$, will instantly experience discontinuous changes in the following Keplerian orbital elements:

- The inclination will instantly shift by $\pi$ such that it is between 0 to $\pi$.

- The RAAN will instantly shift by $\pi$ such that it is between 0 to $\pi$.

- The argument of periapsis will instantly shift by $\pi$ such that it is between 0 to $2 \pi$.

\subsection{2: In-plane thrust only}

In order to maximize $\frac{d \omega}{d t}, a_{R}$ should have the same sign as $-\cos (v)$. As in

4.4: Maximization of the Rate-of-Change of the Semi-Major Axis, letting the radial acceleration be defined as a function of the tangential acceleration,

$$
a_{R}=\sqrt{m a x^{2}-a_{\theta}^{2}} R=\left[m a x^{2}-a_{\theta}^{2}\right]^{\frac{1}{2}} R,
$$

where

$$
R(v)=\operatorname{sign}(-\cos (v)),
$$

results in

$$
\frac{d \omega}{d t}=\frac{1-e^{2}}{e n b}\left[-\cos (v)\left[\max ^{2}-a_{\theta}^{2}\right]^{\frac{1}{2}} R+\left(1+\frac{1}{1+e \cos (v)}\right) \sin (v) a_{\theta}\right] .
$$

Defining an auxiliary function for $\frac{d \omega_{\text {aux }}}{d t}$ that only considers those portions of the function varying with $v$ results in

$$
\frac{d \omega_{\text {aux }}}{d t}=-\cos (v)\left[\max ^{2}-a_{\theta}^{2}\right]^{\frac{1}{2}} R+\left(1+\frac{1}{1+e \cos (v)}\right) \sin (v) a_{\theta} .
$$

A necessary condition for this function to be maximized with respect to the control input, $a_{\theta}$, is that its derivative with respect to $a_{\theta}$ equals zero,

$$
\frac{d}{d a_{\theta}} \frac{d \omega_{\text {aux }}}{d t}=+\cos (v) R\left[\max ^{2}-a_{\theta}^{2}\right]^{\frac{-1}{2}} a_{\theta}+\left(1+\frac{1}{1+e \cos (v)}\right) \sin (v)=0 .
$$

Letting 


$$
D=\left(1+\frac{1}{1+e \cos (v)}\right) \sin (v)
$$

and re-arranging, leads to

$$
\cos (v) R\left[\max ^{2}-a_{\theta}^{2}\right]^{\frac{-1}{2}} a_{\theta}=-D
$$

Multiplying by $\left[\max ^{2}-a_{\theta}^{2}\right]^{\frac{-1}{2}}$ and squaring the equation leads to

$$
\cos ^{2}(v) a_{\theta}^{2}=D^{2} \max ^{2}-D^{2} a_{\theta}^{2} .
$$

Solving for $a_{\theta}^{2}$ yields

$$
a_{\theta}^{2}=\max ^{2} \frac{D^{2}}{\cos ^{2}(v)+D^{2}} .
$$

Inspection of Eq. (25c) reveals that to maximize $\frac{d \omega}{d t}, a_{\theta}$ should have the same sign as $\sin (\theta)$,

$$
a_{\theta}=\max \frac{D \operatorname{sign}(\sin (\theta))}{\sqrt{\left(\cos ^{2}(v)+D^{2}\right)}} .
$$

Plugging this function back into the auxiliary function, $\frac{d \omega_{a u x}}{d t}$, results in

$$
\begin{aligned}
\frac{d \omega_{a u x}}{d t} & =-\cos (v)\left[1-\frac{D^{2}}{\cos ^{2}(v)+D^{2}}\right]^{\frac{1}{2}} R \\
& +\left(1+\frac{1}{1+e \cos (v)}\right) \sin (v) \frac{D \cdot \operatorname{sign}(\sin (\theta))}{\sqrt{\left(\cos ^{2}(v)+D^{2}\right)}}
\end{aligned}
$$

Fig. 35 shows an example of how $\frac{d \omega_{a u x}}{d t}$ varies with $v$ for a given

eccentricity. The Figure illustrates that the greatest rate of change occurs when $v$ is slightly greater than 90 degrees and slightly less than 270 degrees. In fact, these locations are functions of the eccentricity. 


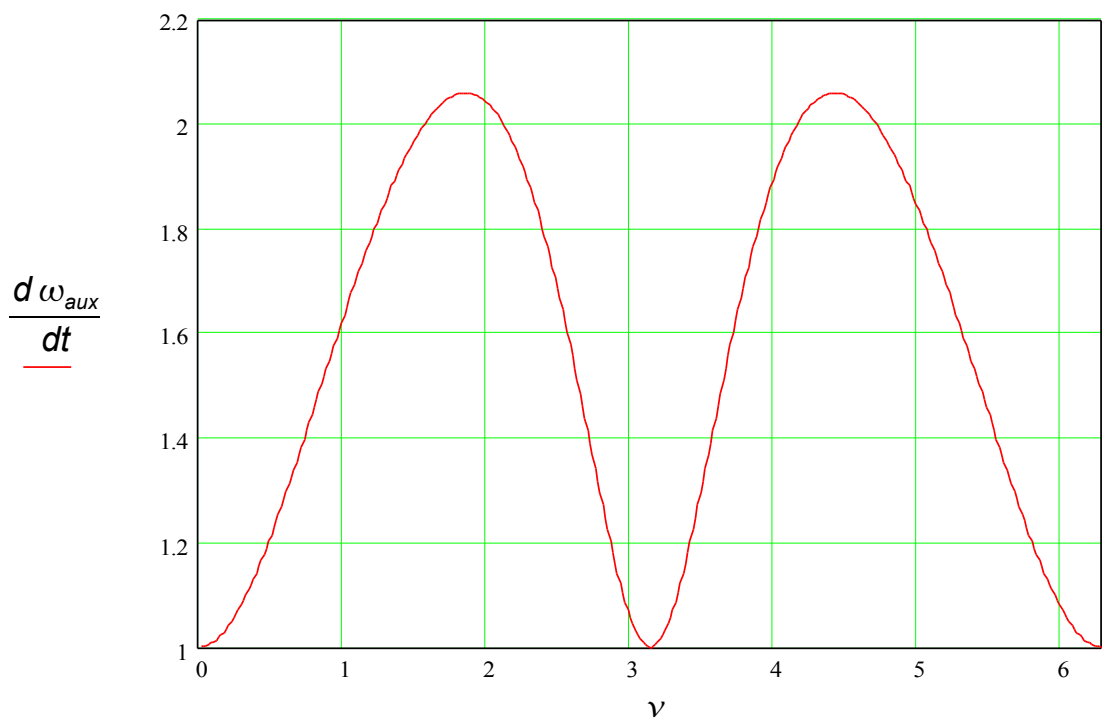

Figure 35 Auxiliary function for the rate of change of the argument of periapsis as a function of true anomaly [rad]. $(e=0.4)$

Fig. 36 shows the corresponding optimal thrusts necessary to achieve the results shown in Fig. 35.

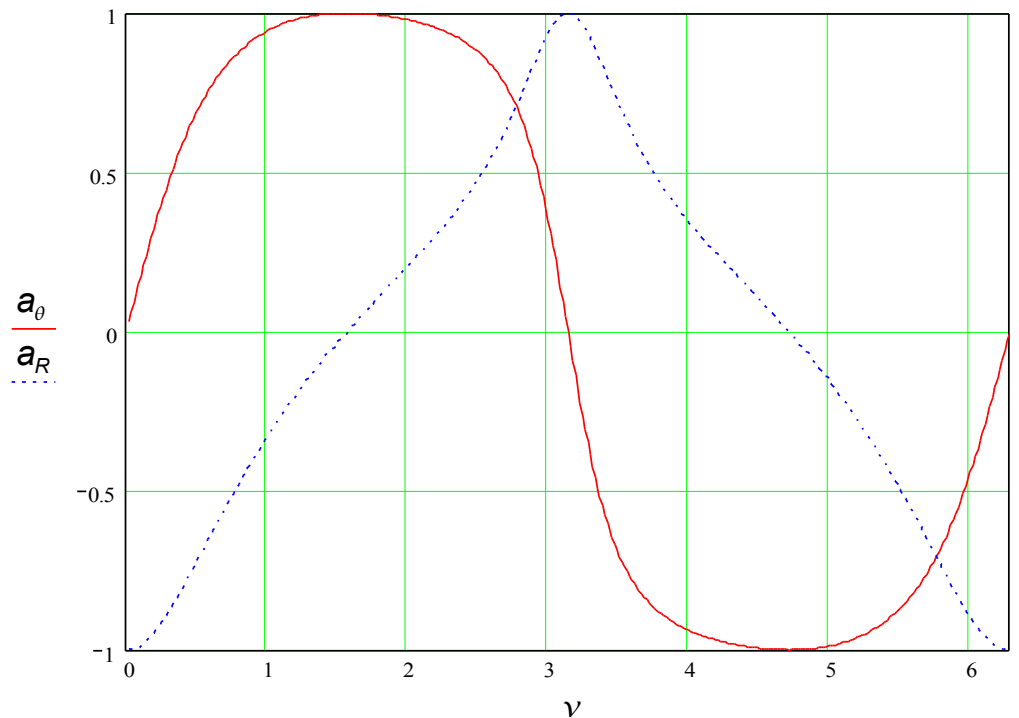

Figure 36 Normalized optimal thrust for maximizing the argument of periapsis $(e=0.4)$

A necessary condition for this function to be maximized with respect to the true anomaly, $v$, is that its derivative with respect to $v$ equals zero; therefore, 


$$
\begin{aligned}
\frac{d}{d v} \frac{d \omega_{a u x}}{d t} & =-\cos (v) \sqrt{\left(1-H^{2}\right)} \frac{d R}{d v}+\frac{\cos (v) R H}{\sqrt{\left(1-H^{2}\right)}} \frac{d H}{d v}+\sqrt{\left(1-H^{2}\right)} R \sin (v) \\
& +C \sin (v) \frac{d H}{d v}+C H \cos (v)+\sin ^{2}(v) H(C-1)^{2} e \\
& =0
\end{aligned}
$$

where

$$
\begin{gathered}
C=1+\frac{1}{1+e \cos (v)}, \\
H=\frac{D}{\sqrt{\left(\cos (v)^{2}+D^{2}\right)}}, \\
\text { and } \quad \frac{d H}{d v}=\frac{\left[\cos (v)^{2}+D^{2}\right]^{\frac{1}{2}} \frac{d D}{d v}-D\left[\cos (v)^{2}+D^{2}\right]^{\frac{-1}{2}}\left(D \frac{d D}{d v}-\cos (v) \sin (v)\right) .}{\cos (v)^{2}+D^{2}} .
\end{gathered}
$$

$\frac{d R}{d v}$ equals zero everywhere except for two points, $\omega+v=\frac{\pi}{2}$ and $\omega+v=\frac{3 \pi}{2}$.

Therefore, the scaling $-\cos (v) \sqrt{\left(1-H^{2}\right)}$ term will be ignored, so

$$
\begin{aligned}
\frac{d}{d v} \frac{d \omega_{\text {aux }}}{d t} & =\frac{\cos (v) R H}{\sqrt{\left(1-H^{2}\right)}} \frac{d H}{d v}+\sqrt{\left(1-H^{2}\right)} R \sin (v)+C \sin (v) \frac{d H}{d v} \\
& +C H \cos (v)+\sin ^{2}(v) H(C-1)^{2} e \\
& =0 .
\end{aligned}
$$

Note that this function is not defined at $\frac{\pi}{2}$ and $\frac{3 \pi}{2}$ because these points are where the first term is equal to $\frac{0}{0}$. 


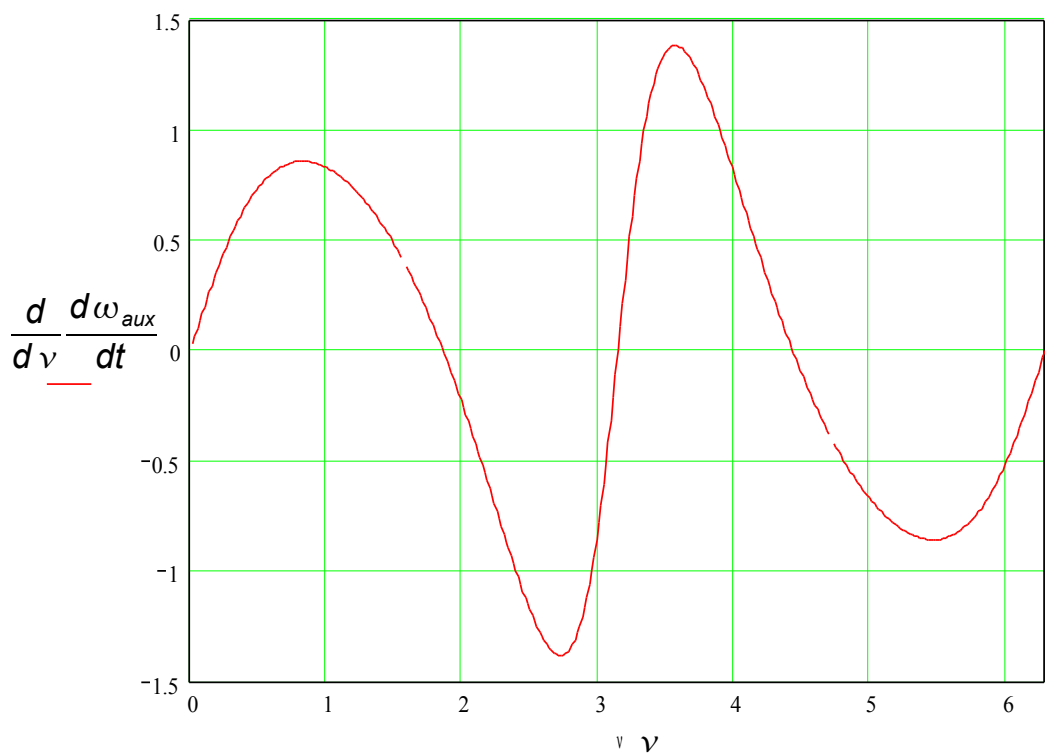

Figure 37 The true-anomaly-derivative of the time rate-of-change of the auxiliary, argument of periapsis as a function of true anomaly [rad] $(e=0.4)$

Therefore, to find exactly where $\frac{d}{d v} \frac{d \omega_{a u x}}{d t}=0$, one can numerically find the root of this new equation. Of particular interest are the solutions strictly within Quadrants II and III; these correspond to the maximums of $\frac{d \omega_{\text {aux }}}{d t}=0$. It is worth emphasizing that the roots of the equations are functions of the orbital eccentricity.

In summary, the control law described by Eq. (39) should be applied within a user-specified, threshold of the roots of Eq. (40) in Quadrants II and III. 


\section{7: Maximization of the Rate-of-Change of the Semi-Major Axis while keeping the Eccentricity constant}

This section seeks to find an orbital maneuver that maximizes the rate-ofchange of the SMA (semi-major axis), a , while keeping the eccentricity, $e$, constant.

In Section 4.4: Maximization of the Rate-of-Change of the Semi-Major Axis, the optimal control law for maximizing the rate-of-change of the SMA was found to be

$$
a_{R}=\sqrt{\max ^{2}-a_{\theta}^{2}} \operatorname{sign}(\sin (v))=\left[\max ^{2}-a_{\theta}^{2}\right]^{\frac{1}{2}} \operatorname{sign}(\sin (v))
$$

and $a_{\theta}=\max \sqrt{\frac{(1+e \cos (v))^{2}}{e^{2}+2 e \cos (\nu)+1}}$.

Recall that the rate-of-change of the eccentricity is

$$
\frac{d e}{d t}=\frac{\left(1-e^{2}\right)}{n b}\left(\sin (v) a_{R}+A a_{\theta}\right) \text {, }
$$

where

$$
A=\left[\left(1+\frac{1}{1+e \cos (v)}\right) \cos (v)+\frac{1}{1+e \cos (v)} e\right]
$$

Inspection of the scaling factor for the $a_{R}$ term, $\sin (v)$, reveals that a radial thrust at $v=0$ or $v=\pi$ would result in no change in the eccentricity. However, this is also where the optimal tangential thrust, $a_{\theta}$, as defined by Eq. (34b), equals $\max$ and therefore where the optimal radial thrust, $a_{R}$, as defined by Eq. (34a), equals 0 . In summary, a radial thrust at this location would have no effect on either the SMA or the eccentricity.

Therefore, consider the scaling factor for the $a_{\theta}$ term, $A$. Solving for where $A=0$ identifies where a tangential thrust, $a_{\theta}$, will have no effect on eccentricity and, therefore, keep it constant.

Multiplying by $1+e \cos (v)$ and re-arranging leads to

$$
e \cos ^{2}(v)+2 \cos (v)+e=0 \text {. }
$$

Let $x=\cos (v)$. Then, by a change of variable, the equation becomes

$$
e x^{2}+2 x+e=0
$$

which is simply a quadratic equation. The solution for $x$ is

$$
x=\frac{-1}{e} \pm \frac{1}{e} \sqrt{\left(1-e^{2}\right)} .
$$

Changing the variable back leads to 


$$
v= \pm \operatorname{acos}\left[\frac{-1}{e} \pm \frac{1}{e} \sqrt{\left(1-e^{2}\right)}\right]
$$

Additionally, the \pm within the arc-cosine can be simplified by only considering the + variant since that is the only variant with a real-numbered solution.

Finally, if the eccentricity is zero, the solution is undefined; this will be handled below as a special condition.

In summary, the maximum tangential thrust should be applied within a userspecified, threshold of the true anomaly defined by Eq. (41) in order to maximize the SMA and keep the eccentricity constant.

\section{Special Condition: Initial Orbit is Circular and Equatorial}

If the initial orbit is circular ( $e=0$ ), the optimal thrusting location defined by Eq. (41) is not defined. In Section 4.4: Maximization of the Rate-of-Change of the Semi-Major Axis, it was observed that positive tangential thrusting increases the SMA. In Section 4.5: Maximization of the Rate-of-Change of Eccentricity, it was observed that eccentricity is increased with positive tangential thrusting near periapsis and negative tangential thrusting near apoapsis. Positive tangential thrusting near apoapsis will decrease eccentricity. Therefore, the strategy to increase the SMA while keeping the orbit nearly circular is to repeatedly:

1. Apply positive tangential thrusting at one location in the orbit. This will increase the SMA while also causing the orbit to become slightly eccentric.

2. Apply positive tangential thrusting near apoapsis, approximately 180 degrees later in the orbit. This will increase the SMA while decreasing orbital eccentricity back to nearly zero.

This strategy is similar to a series of Hohmann orbit transfers 43 .

It is arbitrary where along the orbit the initial thrusting should occur. In this work, the "temporary" periapsis location will be selected where the true longitude equals zero (i.e. aligns with the inertial $X$-axis).

\section{Special Condition: Initial Orbit is Circular and Inclined}

In the case of a circular, inclined orbit, the same argument applies as in the special condition for the circular, equatorial orbits. The only difference is that the periapsis will be located near the argument of latitude of zero (i.e. thrusting will occur near the ascending and descending nodes). 


\section{8: Maximization of the Rate-of-Change of Argument of Periapsis while keeping Eccentricity constant}

This section seeks to find an orbital maneuver that maximizes the rate-ofchange of the argument of periapsis, $\omega$, while keeping the eccentricity, $e$, constant. Note that this maneuver is irrelevant when the initial orbit is circular (e $=0$ ) due to the argument of periapsis not being defined for such orbits.

In Section 4.6: Maximization of the Rate-of-Change of Argument of Periapsis, the optimal, in-plane control was found to be

$$
a_{R}=\sqrt{\max ^{2}-a_{\theta}^{2}} R=\left[\max ^{2}-a_{\theta}^{2}\right]^{\frac{1}{2}} R
$$

and $\quad a_{\theta}=\max \frac{D \operatorname{sign}(\sin (\theta))}{\sqrt{\left(\cos ^{2}(v)+D^{2}\right)}}$,

where

$$
\begin{aligned}
& R(v)=\operatorname{sign}(-\cos (v)) \text { and } \\
& D=\left(1+\frac{1}{1+e \cos (v)}\right) \sin (v)
\end{aligned}
$$

In that same section, the optimal location for applying this optimal control was found to be located at the root of

$$
\begin{aligned}
\frac{d}{d v} \frac{d \omega_{\text {aux }}}{d t} & =\frac{\cos (v) R H}{\sqrt{\left(1-H^{2}\right)}} \frac{d H}{d v}+\sqrt{\left(1-H^{2}\right)} R \sin (v)+C \sin (v) \frac{d H}{d v} \\
& +C H \cos (v)+\sin ^{2}(v) H(C-1)^{2} e \\
& =0
\end{aligned}
$$

where

$$
\begin{gathered}
C=1+\frac{1}{1+e \cos (v)}, \\
H=\frac{D}{\sqrt{\left(\cos (v)^{2}+D^{2}\right)}}, \\
\text { and } \quad \frac{d H}{d v}=\frac{\left[\cos (v)^{2}+D^{2}\right]^{\frac{1}{2}} \frac{d D}{d v}-D\left[\cos (v)^{2}+D^{2}\right]^{\frac{-1}{2}}\left(D \frac{d D}{d v}-\cos (v) \sin (v)\right) .}{\cos (v)^{2}+D^{2}} .
\end{gathered}
$$

Numerical evaluation of this control law applied near the optimal location indicates that the optimal thrusting largely consists of tangential thrusting.

Interestingly, this optimal location is located near to where a tangential thrust has no effect on the eccentricity. As discussed in Section 4.7: Maximization of the 
Rate-of-Change of the Semi-Major Axis while keeping the Eccentricity constant, any tangential thrust applied near

$$
v= \pm \operatorname{acos}\left[\frac{-1}{e} \pm \frac{1}{e} \sqrt{\left(1-e^{2}\right)}\right]
$$

has little to no effect on eccentricity. As was noted earlier, the \pm within the arccosine can be simplified by only considering the + variant since that is the only variant with a real-numbered solution. Even if the eccentricity is zero, the solution is undefined but so is the definition of argument of periapsis, in which case this maneuver is meaningless.

Therefore, a comparison of tangential thrusting at the true anomaly from the root of Eq. (40) versus the true anomaly from Eq. (42) can be made. Fig. 38 shows both true anomalies for ranges of eccentricities between 0.0 and 0.9 . Their difference increases with eccentricity up to a maximum difference of approximately 30 degrees.

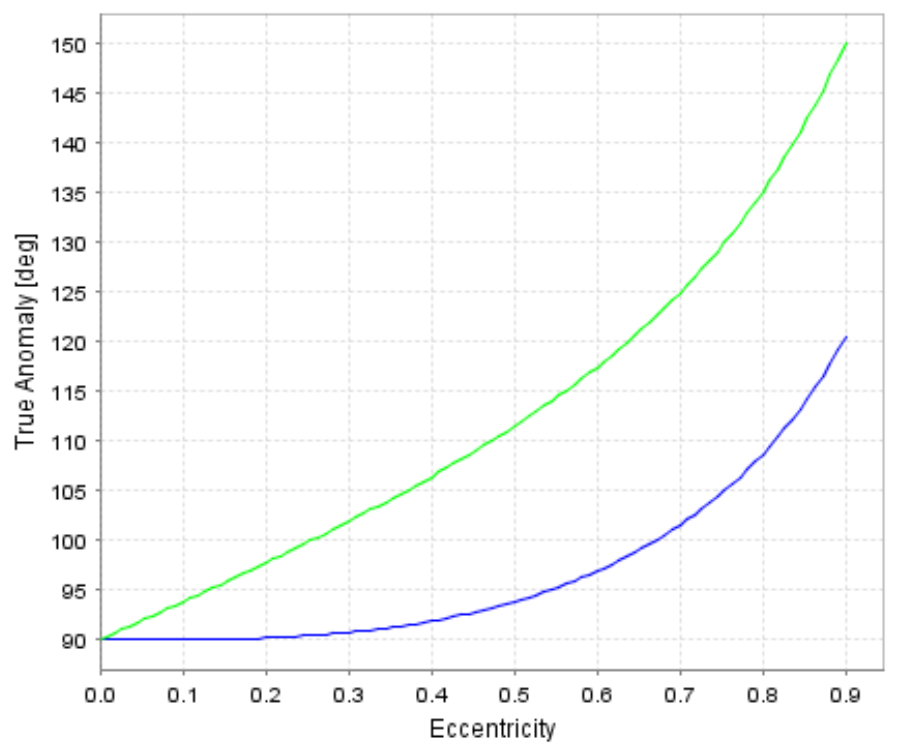

Figure 38: Comparison of the optimal location for maximizing the rate-of-change of the argument of periapsis (green) versus the location where a tangential thrust has no effect on the eccentricity (blue).

Fig. 39 compares the two thrusting strategies. For this figure, efficiency is defined as the rate-of-change of the argument of periapsis compared to the optimal rate-of-change of the argument of periapsis. The green line, at $100 \%$ efficiency, corresponds to optimally thrusting along the optimal locations (green curve in Fig. 38). The blue curve corresponds to tangentially thrusting where eccentricity is not affected (blue curve in Fig. 38). The efficiency drops with everincreasing eccentricity but does not descend below $82 \%$. 


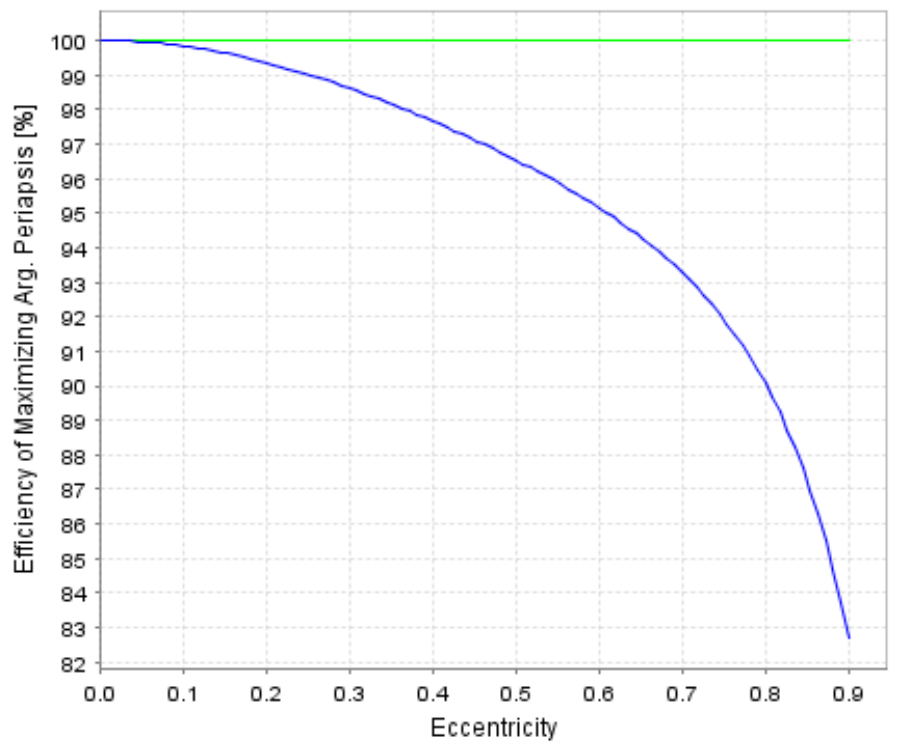

Figure 39: Comparison of the optimal thrusting strategy to maximize the rate-of-change of the argument of periapsis (green) versus tangential thrusting where the eccentricity is not altered (blue.)

In summary, the maximum tangential thrust should be applied within a userspecified, threshold of the true anomaly defined by Eq. (42). Note that this is the same thrusting strategy employed in Section 4.7: Maximization of the Rate-ofChange of the Semi-Major Axis while keeping the Eccentricity constant when the initial orbit is eccentric. 


\section{9: Maximization of the Rate-of-Change of Argument of Periapsis while keeping Semi-Major Axis, Eccentricity, Inclination, and Right-Ascension of the Ascending Node Constant}

This section seeks to find an orbital maneuver that maximizes the rate-ofchange of the argument of periapsis, $\omega$, while keeping the SMA (semi-major axis), $a$, and the eccentricity, $e$, constant. Note that this maneuver is irrelevant when the initial orbit is circular $(e=0)$ due to the argument of periapsis not being defined for such orbits.

Recall some of Gauss' variational equations:

$$
\begin{aligned}
\frac{d \Omega}{d t} & =\frac{1-e^{2}}{n b \sin (i)} \frac{\sin (\omega+v)}{1+e \cos (v)} a_{C T} \\
\frac{d i}{d t} & =\frac{1-e^{2}}{n b} \frac{\cos (\omega+v)}{1+e \cos (v)} a_{C T} \\
\frac{d a}{d t} & =\frac{2 a}{n b}\left(e \sin (v) a_{R}+(1+e \cos (v)) a_{\theta}\right), \\
\frac{d e}{d t} & =\frac{\left(1-e^{2}\right)}{n b}\left(\sin (v) a_{R}+\left[\left(1+\frac{1}{1+e \cos (v)}\right) \cos (\theta)+\frac{1}{1+e \cos (v)} e\right] a_{\theta}\right), \\
\frac{d \omega}{d t} & =\frac{1-e^{2}}{e n b}\left[-\cos (v) a_{R}+\left(1+\frac{1}{1+e \cos (v)}\right) \sin (v) a_{\theta}\right] \\
& -\frac{1-e^{2}}{n b} \frac{\cos (i)}{\sin (i)} \frac{\sin (\omega+v)}{1+e \cos (v)} a_{C T} .
\end{aligned}
$$

To avoid perturbing the right-ascension of the ascending node, $\Omega$, and inclination, $\mathrm{i}$, one approach is to not accelerate out of the orbital plane $\left(a_{C T}=0\right)$. Then, inspection of the equations for $\frac{d a}{d t}, \frac{d e}{d t}$, and $\frac{d \omega}{d t}$, reveals that a radial thrust, $a_{C T}$, near periapsis ( $v=0$ ) or apoapsis ( $v=\pi$ ) will have nearly no effect on $\frac{d a}{d t}$ and $\frac{d e}{d t}$ but will affect $\frac{d \omega}{d t}$. Therefore, the radial thrusting should be defined as

$$
a_{R}=\max \operatorname{sign}(-\cos (v))
$$

for maximization. Fortunately, radial thrusting near the periapsis and apoapsis is actually the $\omega$-maximizing thrusting determined in Section4.6: Maximization of the Rate-of-Change of Argument of Periapsis (See Fig. 36). Unfortunately, inspection of Fig. 35 shows that this is also where the $\omega$-maximizing thrust achieves its weakest effect. 
In summary, the control law described by Eq. (43) should be applied within a user-specified, threshold of the periapsis and apoapsis in order to maximize the rate-of-change of the argument of periapsis, $\omega$, while keeping the SMA, $a$, and the eccentricity, $e$, constant. 


\subsection{0: Maximization of the Rate-of-Change of the Semi-Major Axis and Eccentricity while keeping the Argument of Periapsis Constant}

This section seeks to find an orbital maneuver that maximizes the rate-ofchange of argument of the SMA (semi-major axis), a , and the eccentricity, $e$, while keeping the argument of periapsis, $\omega$, constant. Recall some of Gauss' variational equations:

$$
\begin{aligned}
\frac{d a}{d t}= & \frac{2 a}{n b}\left(e \sin (v) a_{R}+(1+e \cos (v)) a_{\theta}\right), \\
\frac{d e}{d t} & =\frac{\left(1-e^{2}\right)}{n b}\left(\sin (v) a_{R}+\left[\left(1+\frac{1}{1+e \cos (v)}\right) \cos (\theta)+\frac{1}{1+e \cos (v)} e\right] a_{\theta}\right), \\
\frac{d \omega}{d t} & =\frac{1-e^{2}}{e n b}\left[-\cos (v) a_{R}+\left(1+\frac{1}{1+e \cos (v)}\right) \sin (v) a_{\theta}\right] \\
& -\frac{1-e^{2}}{n b} \frac{\cos (i)}{\sin (i)} \frac{\sin (\omega+v)}{1+e \cos (v)} a_{C T} .
\end{aligned}
$$

In order to increase the SMA or the eccentricity, thrusting must be applied within the orbital plane using $a_{R}$ and $a_{\theta}$. In Section, 4.5: Maximization of the Rate-of-Change of Eccentricity, it was determined that periapsis and apoapsis were the optimal locations for increasing the eccentricity. In Section, 4.4:

Maximization of the Rate-of-Change of the Semi-Major Axis, it was determined that the periapsis was the optimal location for increasing the semi-major axis. The optimal location common to both, the periapsis, is also where the optimal thrusting is solely a tangential thrust, $a_{\theta}$. Inspection of Eq. (37) reveals that a tangential thrust near periapsis, $v=0$, will have little effect on the argument of periapsis.

Therefore, a strategy of only tangentially thrusting near periapsis will maximize both the rate-of-change of the SMA and eccentricity while keeping the argument of periapsis, $\omega$, nearly constant. Note that is the same thrusting strategy determined for 4.4: Maximization of the Rate-of-Change of the Semi-Major Axis when the initial orbit is eccentric.

\section{Special Condition: Initial Orbit is Circular and Equatorial}

This special condition is the same special condition determined in Section4.4: Maximization of the Rate-of-Change of the Semi-Major Axis, and will therefore employ the same strategy.

\section{Special Condition: Initial Orbit is Circular and Inclined}

This special condition is the same special condition determined in Section4.4: Maximization of the Rate-of-Change of the Semi-Major Axis, and will therefore employ the same strategy. 


\subsection{1: Minimization of the Rate-of-Change of Any Orbital Parameter}

The strategy to minimize the rate-of-change for any of the parameters previously discussed will be to simply reverse the thrust directions calculated for the maximization maneuvers.

Special Condition: Minimization of the Semi-Major Axis May Cause Eccentricity to Vanish

As discussed in Section 4.4: Maximization of the Rate-of-Change of the SemiMajor Axis, a positive tangential thrust near periapsis will cause the SMA to increase. Consequently, a negative tangential thrust near periapsis will cause the SMA to decrease. However, inspection of the rate-of-change of eccentricity,

$$
\frac{d e}{d t}=\frac{\left(1-e^{2}\right)}{n b}\left(\sin (v) a_{R}+\left[\left(1+\frac{1}{1+e \cos (v)}\right) \cos (\theta)+\frac{1}{1+e \cos (v)} e\right] a_{\theta}\right),
$$

reveals that a negative tangential thrust near periapsis will also decrease the eccentricity. If the eccentricity drops to zero while this maneuver is still underway, the location of the periapsis will no longer be defined, thus requiring the maneuver to be modified. The modified maneuver will simply reverse the thrust direction determined in Special Condition: Initial Orbit is Circular and Equatorial and Special Condition: Initial Orbit is Circular and Inclined within Section 4.7: Maximization of the Rate-of-Change of the Semi-Major Axis while keeping the Eccentricity constant while starting from an eccentricity of zero.

\section{Special Condition: Orbit Becoming Posigrade-Equatorial}

This special condition is the opposite of the one found in Section 4.3: Special Condition: Orbit Becoming Retrograde-Equatorial. By definition, posigradeequatorial orbits have an inclination of 0 degrees. A satellite that is minimizing the inclination of an inclined orbit may cause the inclination to approach 0 degrees. As this happens, the maneuvering will cease so as to not exceed this minimum inclination. (Exceeding this inclination would cause the inclination and the RAAN to switch by 180 degrees.) 


\subsection{2: Maneuvering Strategy Thresholds}

The control strategies presented in this chapter are implemented within a satellite simulation. Several threshold values must be specified in order to determine whether a satellite's orbital geometry satisfies strategy-dependent conditions. These threshold characteristics are described below.

Table 4: Thresholds used by the maneuvering strategies

\begin{tabular}{|l|l|}
\hline \multicolumn{1}{|c|}{ Name } & \multicolumn{1}{|c|}{ Value } \\
\hline $\begin{array}{l}\text { Inclination Threshold } \\
\text { This threshold value is used to determine whether a satellite's orbit is } \\
\text { inclined relative to the equatorial plane and thereby determine } \\
\text { whether certain maneuvering strategies are applicable. }\end{array}$ & 0.1 [deg] \\
\hline $\begin{array}{l}\text { Eccentricity Threshold } \\
\text { This threshold value is used to determine whether a satellite's orbit is } \\
\text { eccentric and thereby determine whether certain maneuvering } \\
\text { strategies are applicable. }\end{array}$ & 0.005 \\
\hline $\begin{array}{l}\text { Angular Proximity to Critical Angle } \\
\text { This tolerance value is used to identify whether a satellite's angular } \\
\text { position is close to a critical maneuvering angle. }\end{array}$ & 1 [deg] \\
\hline $\begin{array}{l}\text { Relative Tolerance } \\
\text { This tolerance value is used to evaluate whether one or two critical } \\
\text { angles should be used with certain maneuvering strategies. The rate- } \\
\text { of-change of a particular orbital property is evaluated at both critical } \\
\text { angles. If both rates-of-change are within the tolerance value, then } \\
\text { they are both used. Otherwise, the critical angle with the greater rate- } \\
\text { of-change is used. }\end{array}$ & $10 \%$ \\
\hline $\begin{array}{l}\text { Minimum Orbital Safety Radius } \\
\text { This threshold value is used as the minimum orbital radius in which a } \\
\text { spacecraft is permitted to operate. This is applicable to certain } \\
\text { maneuvers (e.g. when applying the SMA minimization control strategy } \\
\text { near apoapsis) where the application would drop the periapsis to a } \\
\text { potentially dangerous altitude. }\end{array}$ & \\
\hline
\end{tabular}




\subsection{3: Summary}

The maneuvering strategies in this section described how to perform maximal changes to orbital geometry using onboard thrusting. Although not exhaustive, these strategies do describe how to change the primary orbital parameters. Some criticisms of the approach taken in determining these maneuvers are acknowledged here:

(a) The derivations ignore all other perturbation forces (e.g. J2 gravity and atmospheric drag) under the argument that the propulsive force is much greater.

(b) The derivations ignore the fact that the satellite's mass is changing while thrusting. As a result, the effect of thrusting is stronger towards the end of a maneuver than it is towards the beginning.

(c) The maneuvers require the satellite to thrust along time-varying directions relative to a local-horizontal local-vertical reference frame. This may prove to be problematic in implementation depending on the attitude control capabilities of the satellite.

(d) The maneuvers ignore the effect of thrusting on the sixth orbital parameter, mean anomaly, $M$ (which is directly related to the true anomaly, $v$ ). Depending on the maneuver, this can alter $\dot{M}$ as described by Eq.(25f). Specifically, a maneuver may cause the satellite's mean anomaly to advance or retreat relative to the unperturbed motion of $n$. Therefore, if attempting to thrust in the vicinity of a particular mean anomaly, $M_{\text {crit }}$, and if the thrusting is strong enough, $\dot{M}$ may become negative and thereby cause the mean anomaly, $M$, to retreat from the thrusting region surrounding $M_{\text {crit }}$. Therefore, the location of the satellite along its changing/osculating orbit must be checked periodically to ensure that thrusting is only performed in proximity of the critical angle. As a result, the thrusting may repeatedly turn on and off in a sputtering manner.

Recall that many of the derivations assume thrusting at exactly certain mean or true anomalies. The thrusting implementation in this work determines proximity on either side of a critical angle to provide symmetric control around it. Therefore, ignoring the effect on the mean anomaly will slightly violate the assumptions of many of the maneuvers and, in the case of maneuvers that hold certain parameters constant, will slightly alter those constant parameters. 


\section{Chapter 5: Optimizing Constellation Coverage by Performing Maximal Changes to Orbital Geometry}

\section{1: Rephrasing the Optimization Problem}

The aim of this work is to improve coverage of selected Earth targets by reconfiguring an existing satellite constellation. The optimization problem that described this was previously stated in Section 1.1: Focus of This Work.

One of the greatest difficulties of this optimization problem is finding and phrasing the time-varying thrust vector for each satellite, $\vec{F}(t)$. This optimization parameter can be greatly simplified by using the conclusions from the previous chapters. Chapter 3: Estimating Satellite Coverage showed how a satellite's orbital geometry is directly related to the coverage that it provides.

Chapter 4: Maneuvering Strategies for Maximal Changes in Orbital Geometry identified a set of maneuvering strategies that perform maximal changes to orbital geometry. This suggests using maximal changes to orbital geometry as simpler optimization parameters for the problem. Specifically, the parameters for each satellite will consist of any one of the maneuvering strategies described in Chapter 4, denoted by Mvr, plus a disambiguation angle, denoted by $\alpha$, which is required for certain combinations of maneuvers and satellite orbits ${ }^{H}$.

There still remains one concern related to the satellite maneuvering: how much maneuvering should take place? Maneuvering is limited by the amount of available propellant. However, it is not clear that using all available propellant will yield the best improvement in coverage for all targets. Therefore, propellant allotment for the maneuver will be added into the optimization as an optimization parameter and denoted by $m_{a p}$. Relatedly, the total propellant allocated for the constellation will be added as an additional objective. The transformed optimization problem is qualitatively described in Table 5.

$\mathrm{H}$ Using vehicle maneuvering strategies as optimization parameters was also used by Vivona et al. 44 to identify maneuvers that resolve aircraft trajectory conflicts. This work's "maneuvering strategy" is that work's "pattern". 
Table 5: Qualitative description of the rephrased coverage optimization problem

\begin{tabular}{|c|c|}
\hline Optimize & $\begin{array}{l}\text { - Coverage of Target } 1 \\
\text { - Coverage of Target } 2 \\
\vdots \\
\text { - Coverage of Target } n \\
\text { - Total propellant allocated for maneuvering }\end{array}$ \\
\hline By varying & $\begin{array}{l}\text { - Satellite } 1 \\
\text { - Discrete maneuvering strategy } \\
\text { - Disambiguation angle for the discrete } \\
\text { maneuvering strategy } \\
\text { - Propellant allocated for maneuver } \\
\text { - Satellite } 2 \\
\text { - Discrete maneuvering strategy } \\
\text { - Disambiguation angle for the discrete } \\
\text { maneuvering strategy } \\
\text { - Propellant allocated for maneuver } \\
\vdots \\
\text { - Satellite m } \\
\text { - Discrete maneuvering strategy } \\
\text { - Disambiguation angle for the discrete } \\
\text { maneuvering strategy } \\
\text { - Propellant allocated for maneuver }\end{array}$ \\
\hline $\begin{array}{c}\text { Subject to } \\
\text { (implicit by } \\
\text { formulation) }\end{array}$ & $\begin{array}{l}\text { - Timespan of interest } \\
\text { - Initial conditions of Targets and Earth } \\
\text { - Targets and Earth equations-of-motion } \\
\text { - Initial conditions of satellite } \\
\text { - Satellite equations-of-motion } \\
\text { - Line-of-sight constraints } \\
\text { - Limits on satellite propulsion } \\
\text { - Finite thrust limits } \\
\text { - Finite propellant }\end{array}$ \\
\hline
\end{tabular}

The following shows the same optimization problem phrased in a more mathematical form.

Maximize $\quad \operatorname{Cvg}^{s}\left(t_{0}, t_{f}, \vec{S}, e l, \overrightarrow{\operatorname{Conx}}\left(t_{0}, \overrightarrow{M v r}(\vec{\alpha}), \vec{m}_{a p}\right)\right)$ for $s=1 \cdots S$

and minimize $\sum_{r=1}^{R} m_{p}^{r}\left(t_{0}\right)-m_{p}^{r}\left(t_{f}\right)$

by varying $\left[\begin{array}{c}M v r^{r} \\ \alpha^{r} \\ m_{a p}^{r}\end{array}\right]$ for $r=1 \cdots R$

subject to $\quad m_{p}^{r}\left(t_{0}\right)=m_{a p}^{r}$ for $r=1 \cdots R$,

$$
0 \mathrm{~kg} \leq m_{p}^{r} \leq m_{a p}^{r} \leq m_{m p}^{r}<m_{\text {sato }}^{r} \text { for } r=1 \cdots R \text {, }
$$




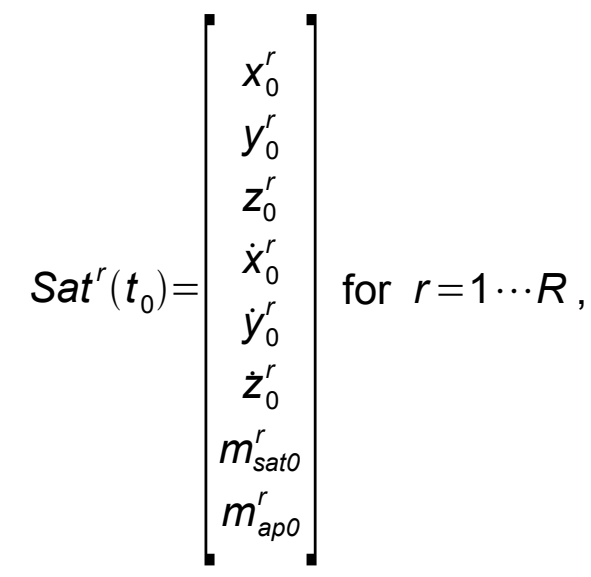

$\sqrt{x^{r}(t)^{2}+y^{r}(t)^{2}+z^{r}(t)^{2}} \geq$ radius $_{\min }>E R$ for $r=1 \cdots R$,

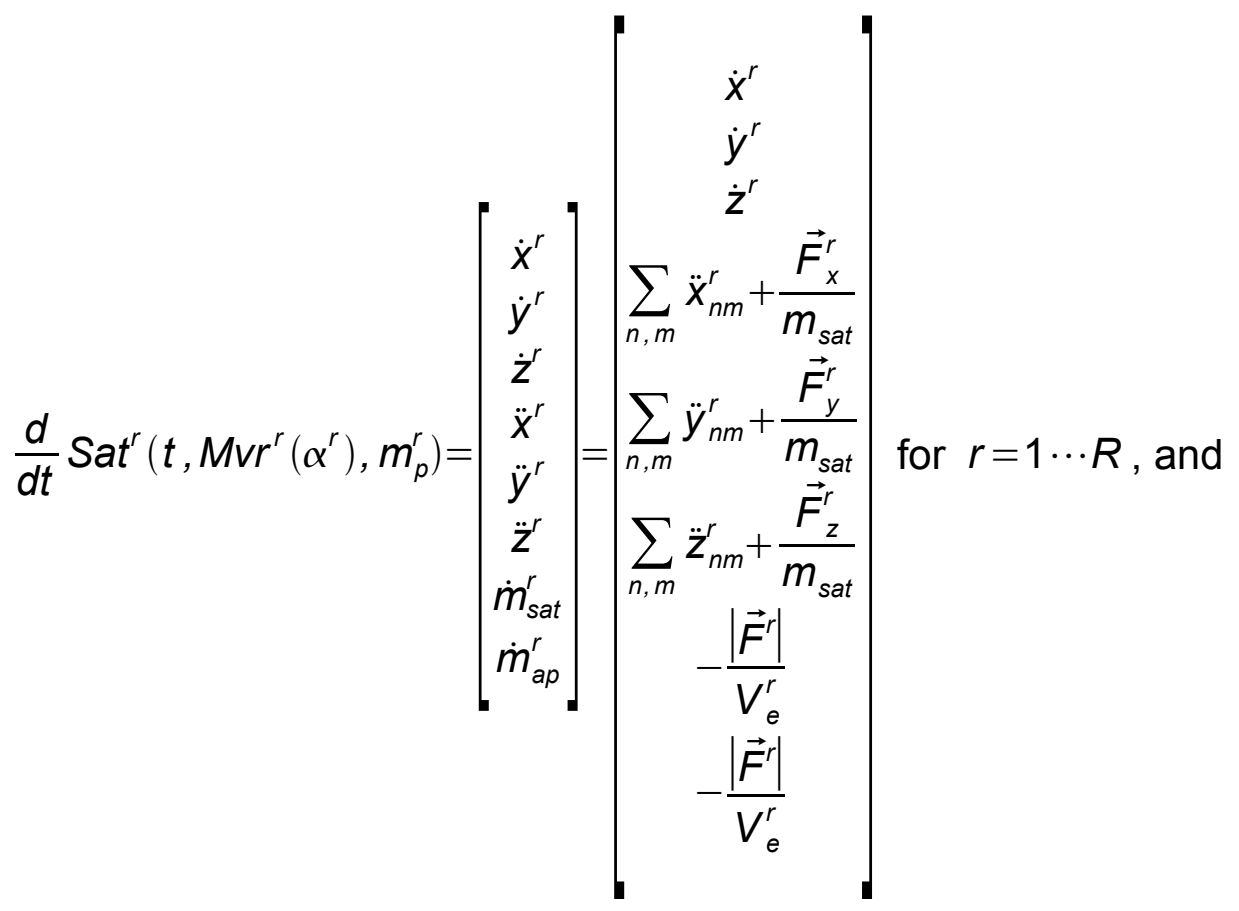

$\left|\vec{F}^{r}\left(\operatorname{Sat}^{r}(t), \operatorname{Mvr}^{r}\left(\alpha^{r}\right)\right)\right| \leq F_{\max }$ for $r=1 \cdots R$,

where

$$
\vec{F}^{r}\left(\operatorname{Sat}^{r}(t), \operatorname{Mvr}^{r}\left(\alpha^{r}\right)\right)=\left[\begin{array}{c}
F_{x}^{r}\left(\operatorname{Sat}^{r}(t), \operatorname{Mvr}^{r}\left(\alpha^{r}\right)\right) \\
F_{y}^{r}\left(\operatorname{Sat}^{r}(t), \operatorname{Mvr}^{r}\left(\alpha^{r}\right)\right) \\
F_{z}^{r}\left(\operatorname{Sat}^{r}(t), \operatorname{Mvr}^{r}\left(\alpha^{r}\right)\right)
\end{array}\right] \text { for } r=1 \cdots R,
$$




$$
\begin{aligned}
& \operatorname{Sat}^{r}(t)=\left[\begin{array}{c}
x^{r}(t) \\
y^{r}(t) \\
z^{r}(t) \\
\dot{x}^{r}(t) \\
\dot{y}^{r}(t) \\
\dot{z}^{r}(t) \\
m_{\text {sat }}^{r}(t) \\
m_{p}^{r}(t)
\end{array}\right] \text { for } r=1 \cdots R ; \\
& \overline{\operatorname{Conx}}(t)=\left[\begin{array}{c}
\operatorname{Sat}^{1}(t) \\
\operatorname{Sat}^{2}(t) \\
\vdots \\
\operatorname{Sat}^{R}(t)
\end{array}\right] ; \\
& t_{0} \leq t \leq t_{f} ;
\end{aligned}
$$

$S$ is the number of targets; $\mathrm{Cvg}^{s}$ is a scalar function representing the coverage provided by the satellite constellation over target $s$ (see Section 2.3: Determining Visibility between Satellites and Targets); $\vec{S}$ is a vector representation of the target locations on the surface of the Earth; $t_{0}$ is the initial time of the scenario; $t_{f}$ is the final time of the scenario; $t$ is a moment in time in the scenario; $R$ is the number of satellites in the constellation; $r$ is the index of a particular satellite; el is the minimum elevation angle above the local horizon below which any satellite is not visible from a target; $M v r^{r}$ represents the discrete maneuvering strategy for satellite $r$ (see Chapter 4: Maneuvering Strategies for Maximal Changes in Orbital Geometry); $\alpha^{r}$ represents the disambiguation angle for satellite $r$ 's maneuvering strategy (which is only used by the maneuvering strategy if the strategy requires it'); $\overrightarrow{M v r}$ is a vector function containing the discrete maneuvering strategies for every satellite in the constellation; $\vec{\alpha}$ is a vector containing the disambiguation angles for every satellite in the constellation; $m_{a p}^{r}$ is the amount of propellant mass allocated for executing $\mathrm{Mvr}^{r} ; \mathrm{m}_{m p}^{r}$ is the maximum amount of propellant mass that can be allocated for maneuvering satellite $r ; \vec{F}^{r}$ is a three-dimensional, vector function representing the propulsive vector for satellite $r ; \vec{F}_{x}^{r}, \vec{F}_{y}^{r}$, and $\vec{F}_{z}^{r}$ are the Cartesian components of $\vec{F}^{r} ; F_{\max }^{r}$ is the maximum thrusting capability of satellite $r ; m_{\text {sato }}^{r}$ is the initial mass of satellite $r ; m_{\text {sat }}^{r}$ is the instantaneous mass

I For example, if the maneuvering strategy calls for increasing the eccentricity of an orbit and the initial orbit is circular, then a disambiguation angle is required to specify where the periapsis should initially be placed. However, if the initial orbit is not circular then its periapsis is already defined and hence the disambiguation angle can be ignored. 
of satellite $r$; ER is the Earth's mean radius; radius min $_{\text {in }}$ is the minimum orbital safety radius below which any satellite should not maneuver; $x_{0}^{r}, y_{0}^{r}$, and $z_{0}^{r}$ represent the initial Cartesian position of satellite $r ; \dot{x}_{0}^{r}, \dot{y}_{0}^{r}$, and $\dot{z}_{0}^{r}$ represent the initial Cartesian velocity of satellite $r ; \dot{x}^{r}, \dot{y}^{r}$, and $\dot{z}^{r}$ represent the instantaneous Cartesian velocity of satellite $r ; \ddot{x}^{r}, \ddot{y}^{r}$, and $\ddot{z}^{r}$ represent the instantaneous Cartesian acceleration of satellite $r ; \ddot{x}_{n m}^{r}, \ddot{y}_{n m}^{r}$, and $\ddot{z}_{n m}^{r}$ represent the various terms of the spherical harmonic, gravitational model for Earth (see Section 2.1: Motion of Satellites in Orbit); Sat ${ }^{r}$ is the instantaneous state of satellite $r$; and $\overrightarrow{\operatorname{Conx}}$ is a vector function representing the instantaneous state of each satellite in the constellation.

Recall that the original problem phrased in Section 1.1: Focus of This Work optimized multiple coverage objectives by varying a time-dependent, threedimensional, propulsive vector for each satellite (i.e. $\overrightarrow{F^{r}}(t)$ ). In contrast, the newly phrased problem bears two significant differences.

(a) Another objective has been added to the problem which seeks to minimize the total propellant consumed by the satellite constellation.

(b) The new problem varies a simpler set of parameters for each satellite (i.e. one discrete variable, $M v r^{r}$, and two continuous variables, $\alpha^{r}$ and $\left.m_{a p}^{r}\right)$ in order to optimize the various objectives. The old optimization parameter, $\vec{F}^{r}(t)$, is now a partial function of the new parameters, $M v r^{r}$, $\alpha^{r}$ and $m_{a p}^{r}$. In effect, $\overrightarrow{F^{r}}(t)$ will now be constrained to a subset of the original feasible region.

Despite these changes, the optimization problem still bears certain difficulties:

(a) The optimization parameters are discrete and continuous

For each satellite, there is one discrete variable and two continuous variables.

(b) Nonlinear objectives

The objective functions are one of the figures-of-merit discussed earlier in Section 2.3: Determining Visibility between Satellites and Targets. These figures-of-merit tend to be discontinuous with respect to changes in the orbit due to coverage windows popping in and out of the visibility schedule.

(c) Multiple objectives

The optimization problem seeks to improve coverage of multiple targets. However, depending on the targets and the satellite constellation, improving the coverage over one target may actually worsen the coverage over another. Therefore, there will not likely be a single solution that optimizes all of the objectives. 
Recall the coverage objectives, $\mathrm{Cvg}^{\mathrm{s}}$, are defined by the figures of merit from Section 2.3: Determining Visibility between Satellites and Targets which are themselves functions of the target visibility schedules. However, since there is no known analytical representation of a visibility schedule, neither is there a known analytical representation of the coverage functions, let alone an analytical solution to this optimization problem. Therefore, this work has pursued finding a solution with a numerical approach.

Not many optimization algorithms can handle all of the difficulties posed by this problem. Nevertheless, one algorithm that is well suited to accommodate this optimization problem, without any further transformation, is a multiobjective evolutionary algorithm. There are several incarnations of such an algorithm and the specific implementation used in this work will be discussed in the following sections.

\section{2: Evolutionary Algorithms for Optimization}

\subsection{1: Single-Objective Evolutionary Algorithms}

Genetic Algorithms 45 describe a set of optimization techniques that imitate biological evolutionary processes by taking good designs for an organism, system, etc. and blending them together to hopefully obtain better designs. Parameters for an optimization problem are classified as genes. The set of all genes that entirely describes the parameters of a problem is classified as a chromosome. A set of chromosomes, representing different solutions, is classified as a population. The quality of a particular chromosome is evaluated using a fitness function, just like a figure-of-merit evaluates the quality of a particular constellation. Genetic Algorithms evaluate the fitness of each chromosome in the population, create new chromosomes from this population, and keep the best from the entire lot, thus imitating "survival of the fittest" 46 . Genetic Algorithms typically employ the following steps 47 :

1.Initialization: An initial population/generation of chromosomes is randomly created. The fitness of each chromosome is evaluated.

2.Partial Population of the Next Generation with the Prior Generation (Elitism): A subset of the prior generation automatically survives to the next generation based on their superior fitness.

3.Parent Selection: A pair of chromosomes are selected from the prior generation.

4.Child Creation (Cloning and Crossover): The selected pair breed and create two children that are some combination of the parents.

5. Child Mutation: Some fraction of the children are randomly mutated to bring an additional level of diversity to the population.

6.Partial Population of the Next Generation with Children: The fitness of the children are evaluated, and placed into the new generation. 
Steps 3 through 6 are repeated to fill in those vacant slots in the next generation that were left open after the elite chromosomes were added.

Genetic Algorithms make no claim as to the global or local optimality of the solutions found. In fact, it is possible for an entire population to get stuck around a particular solution in the parameter space (i.e. "genetic drift"48). Chromosome crossover and chromosome mutation are two operations that attempt to circumvent getting stuck at local minimums. Furthermore, genetic algorithms are quite useful at dealing with fitness functions that are discontinuous with respect to the genes/parameters since the algorithms are ambivalent regarding the continuity of the functions.

\subsection{2: Multi-Objective Evolutionary Algorithms (MOEA)}

The goal of this paper is to optimize constellations that are assessed by multiple figures-of-merit. Attempting to optimize multiple functions with respect to a common set of variables is a multi-objective optimization problem. Optimality in multiobjective problems is assessed by the definition of Pareto Optimality and Weak Pareto Optimality50 (see Fig. 40). A solution is Pareto Optimal when one objective can only be improved at the expense of another objective; such a solution is also called nondominated. A solution is weakly Pareto optimal when no other point can improve all of the objectives. The set of Pareto Optimal points is a subset of the Weakly Pareto Optimal set ${ }^{50}$.

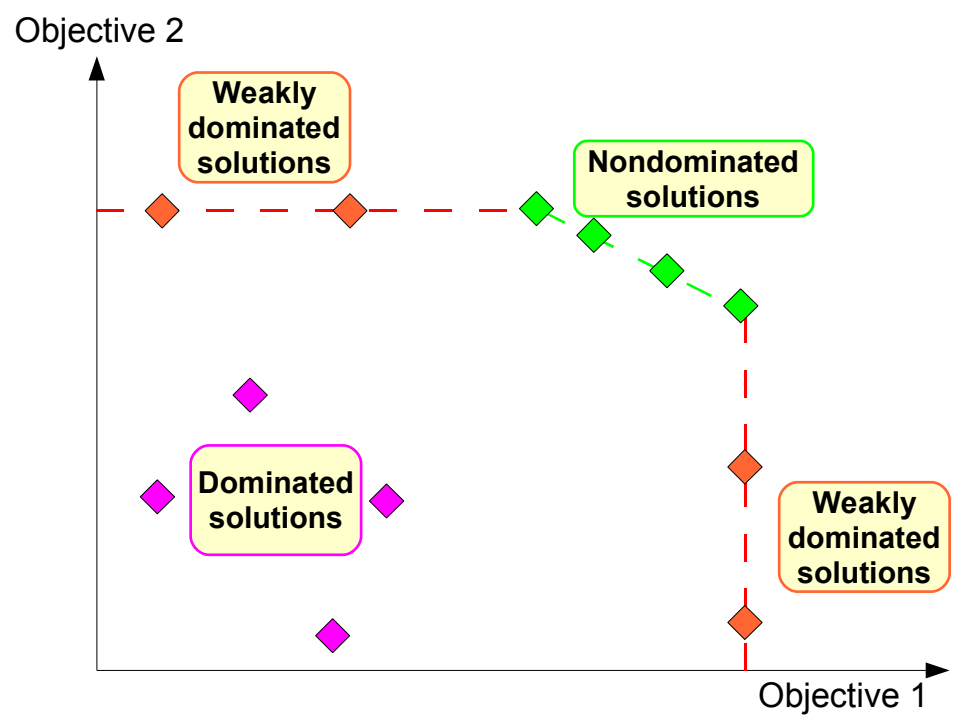

Figure 40: Objective-space depiction of various solutions to a multiobjective maximization problem.

$\mathrm{J}$ The term "Multi-Objective Evolutionary Algorithm" is being used to describe the various implementations of a multiobjective genetic algorithm. This is being done to avoid confusion with one specific implementation called the "Multi-Objective Genetic Algorithm" 49. 


\subsection{3: Implementation of a Multiobjective Evolutionary Algorithm}

This section describes the implementation of the multiobjective evolutionary algorithm used for this work. It is a novel implementation that incorporates several features from other evolutionary algorithms. The pseudo-code for the algorithm is shown in Table 6.

Table 6: Pseudo-code for the implemented multiobjective evolution algorithm

1. Initialize population

2. Evaluate objective values

3. ... until a stopping criterion is satisfied

4. Select parents

5. Create child (crossover and cloning)

6. Mutate child

7. Partially populate the next generation with nondominated solutions

8. Find the nondominated solutions among the merger of the parent generation with the children, and transfer them to the new generation while giving preference to those solutions that maintain greater objective-space separation

9. Partially populate the next generation with only children

10.Find the nondominated solutions among the children, and transfer them to the new generation while giving preference to those solutions that maintain greater objective-space separation

\section{Encoding/Representation of Chromosomes and Genes}

Originally, genetic algorithms converted the parameters of interest into a series of 1 's and 0 's (i.e. binary coding ${ }^{51}$ ). During chromosome crossover and mutation, the 1's and 0's of one chromosome are combined and mutated with those of another. Other representations have also included representing parameters by strings and real-valued numbers. However, another approach can be taken that does not require transforming the original parameters. Each parameter can be represented as a gene that has its own logic for the appropriate genetic operations such as crossover (Step 4) and mutation (Step 5). The benefit of this approach is that parameters can then be represented in whatever form is intuitive and convenient to the software programmer using the optimization software. That is the approach taken in this work. 


\section{Application of Encoding to This Work}

Both chromosomes and genes are represented as objects of the kind available in any object-oriented programming language. Each chromosome contains a pair of genes for each satellite in the constellation (see Fig. 41): a maneuvering strategy gene, and a propellant gene. A maneuvering strategy gene contains any one of the distinct maneuvering strategies that are described in Chapter 4: Maneuvering Strategies for Maximal Changes in Orbital Geometry. Depending on the maneuver, the gene may also contain a continuous parameter representing an angle normalized between 0 and $2 \pi$. This additional parameter is necessary for those maneuvers that are ambiguous without the specification of an initial angle (e.g. specifying the initial RAAN when increasing the inclination of an equatorial orbit, specifying the initial argument of periapsis when increasing the eccentricity of a circular orbit, etc.) The propellant gene contains a continuous parameter representing the amount of propellant to be consumed during the execution of the maneuvering strategy. In summary, each chromosome contains the maneuvering strategies and propellant allotments for all of the constellation satellites, and therefore, represents a solution to the multiobjective optimization problem. 


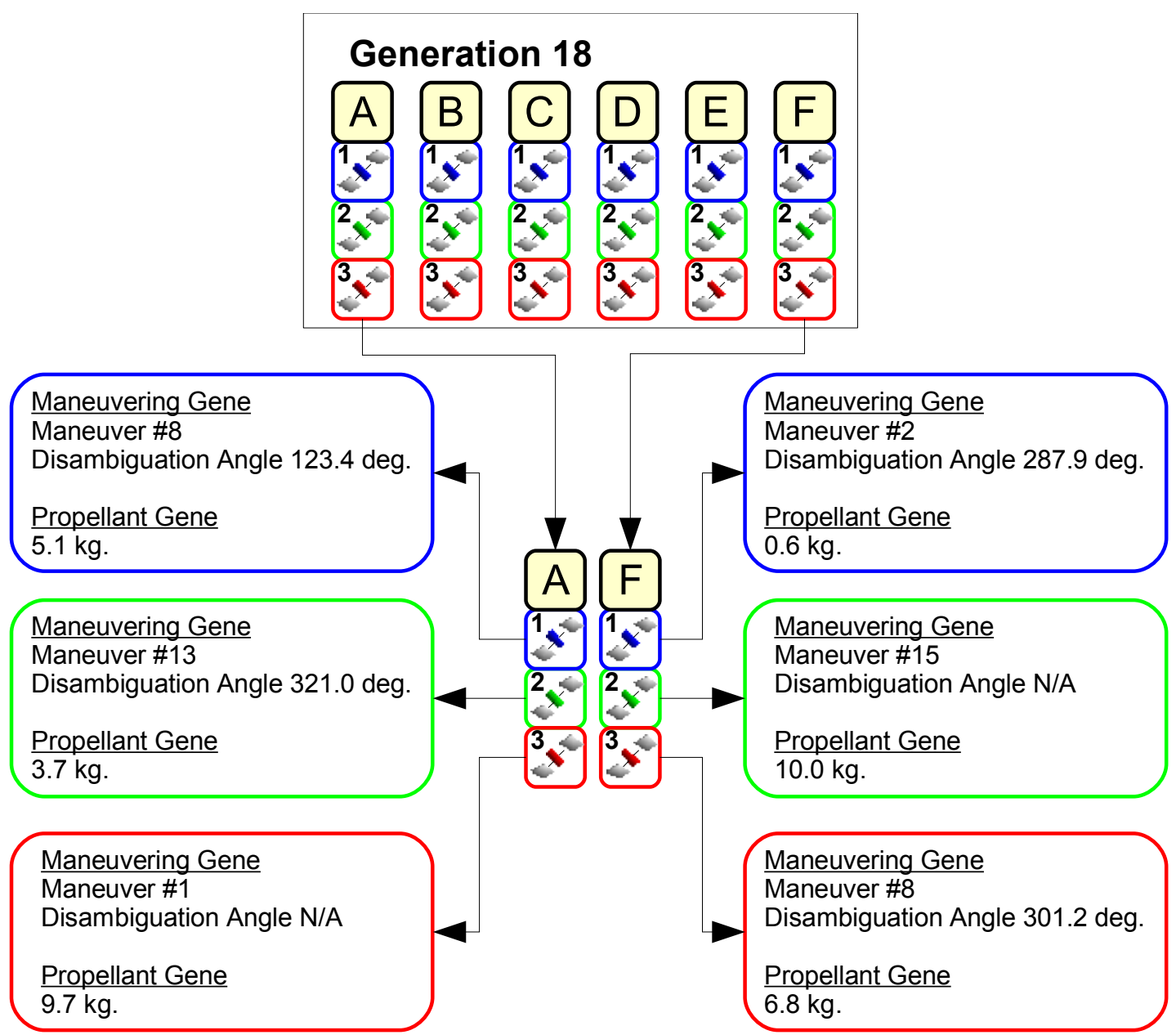

Figure 41: Depiction of a population of chromosomes that represent the maneuvering and propellant genes used in this work. Each chromosome contains a set of genes for each satellite in the constellation. For each satellite, there exists a maneuvering gene and a propellant gene. The maneuvering gene contains a discrete maneuvering strategy as well as a continuous angle that is required for some strategies. The propellant gene contains a continuous variable representing the maximum amount of propellant that may be used by the satellite while executing the maneuvering strategy.

\section{Parent Selection}

In an effort to maintain genetic diversity, each parent is selected from either of two sets of chromosomes. The first set is simply the entire population. The second set is more restrictive and only consists of the nondominated members of the population.

Each parent is selected in a two-step process.

1. One of the two sets is randomly selected.

2. A parent is randomly selected from the set. 
Selecting parents from the nondominated set tends to favor the better solutions. Selecting parents from the entire population tends to maintain genetic diversity by permitting dominated solutions to also be selected.

Random selection from either set reduces any possible bias towards those chromosomes that are best with respect to any individual fitness. This is a criticism of some algorithms such as the Vector Evaluated Genetic Algorithm52, which evenly allocates $\frac{1}{S}$ of succeeding populations from the best portions of a population selected from $S$ objectives. This tends to not fill in the concave portions of the nondominated front 53 .

\section{Child Creation (Crossover and cloning)}

A pair of child chromosomes are produced from a pair of parents by one of two mechanisms: cloning or crossover. Crossover occurs when a random probability exceeds a pre-specified crossover threshold probability; cloning occurs the rest of the time.

\section{Cloning}

During cloning, the pair of child chromosomes are genetic replicas of the parent chromosomes.

\section{Crossover}

During crossover, the pair of child chromosomes are a blend of the parent chromosomes. Each chromosome contains an ordered list of genes. The gene in Slot i of the first chromosome is mated with the gene in Slot $i$ of the second chromosome.

This mating of genes is repeated until two new lists of child genes are generated. At this point, the list of child genes are intermixed at multiple crossover points. The minimum number of crossover points is one. The maximum number of crossover points is one less than the number of genes. Each crossover point is randomly selected. Finally, two child chromosomes are created from the two ordered lists of genes.

\section{Application of Crossover to This Work}

The mating of any maneuvering genes and propellant genes are depicted in Fig. 42. When crossing over maneuvering genes with a disambiguation angle, the child maneuvering genes are generated as follows:

- Child Gene 1 obtains a copy of the orbital maneuvering strategy from Parent Gene 1

- Child Gene 1 obtains a copy of the real-valued angle from Parent Gene 2

- Child Gene 2 obtains a copy of the orbital maneuvering strategy from Parent Gene 2

- Child Gene 2 obtains a copy of the real-valued angle from Parent Gene 1 
When crossing over maneuvering genes with a disambiguation angle, the child genes are simple replicas of the parent maneuvering genes. When crossing over propellant genes, the child genes are also simple replicas of the parent propellant genes. Because of these exact replicas, it is possible for a child chromosome to consist of exact duplicates of one of the parent genes. If the chromosome does not undergo mutation and become unique then the duplicate chromosome will be detected and removed from further consideration (see Section 5.2.3: Guaranteeing Unique Children). 


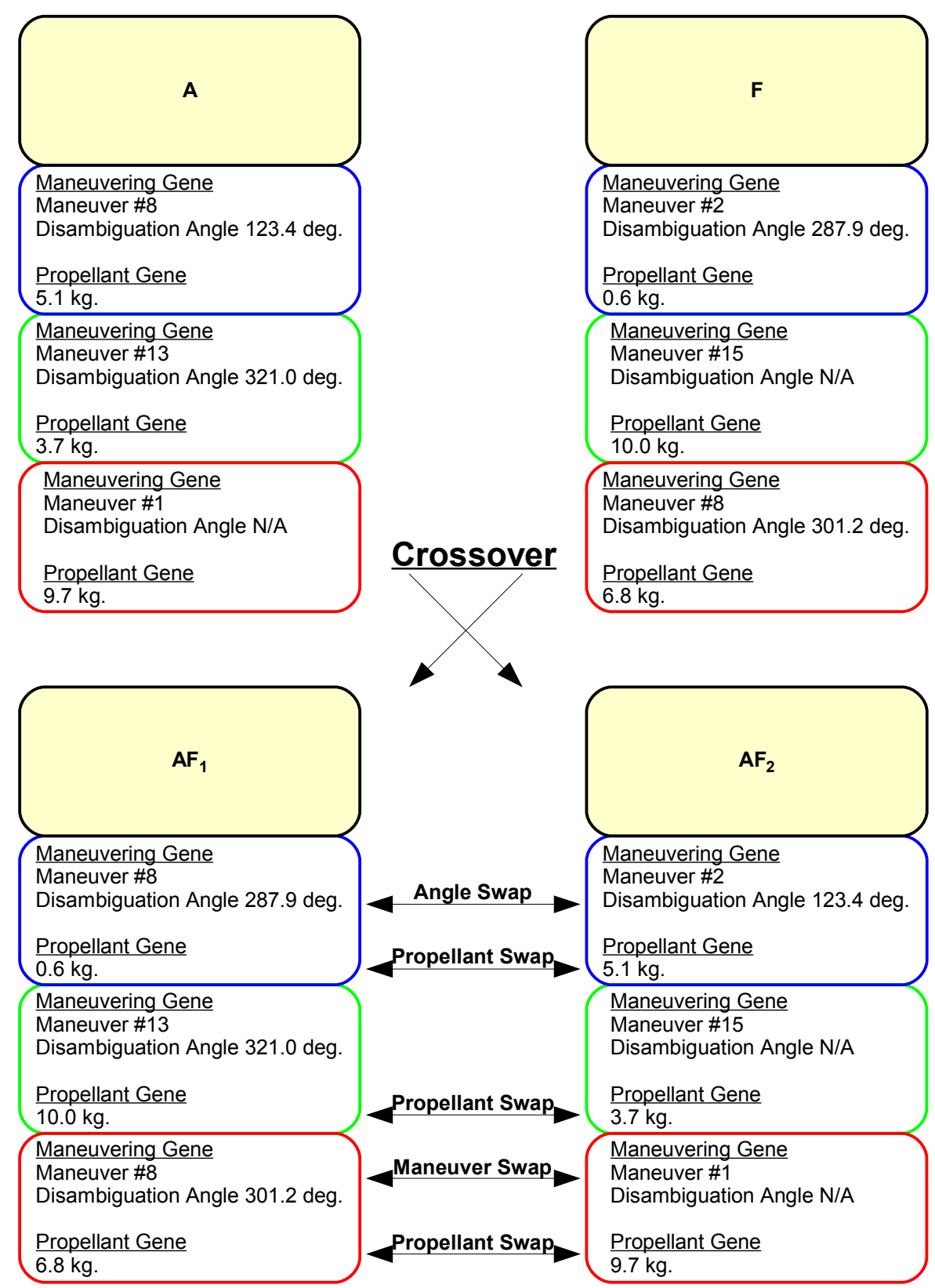

Figure 42: Depiction of the crossover genetic operator applied to two chromosomes used in this work. Given a pair of parent chromosomes, another pair of child chromosomes is created. The parent genes for a particular satellite are crossed-over in order to produce two child genes for that satellite. Some randomization in the crossover determines the level of crossover that occurs. In some cases (as shown for the red satellite), the maneuvering gene and propellant gene remain together. In some cases (as shown for the green satellite), the propellant swaps and pairs with the other maneuvering gene. And in some cases (as shown for the blue satellite), the propellant gene swaps along with the disambiguation angle for the maneuvering strategy. 


\section{Child Mutation}

Mutation of a chromosome only occurs when a random probability exceeds a pre-specified mutation threshold probability. In such cases, a random number of genes (ranging in value between one and the total quantity of genes) are mutated (see Fig. 43). Two forms of mutation are possible, large and small mutations.

\section{Application of Child Mutation to This Work}

During a large mutation to a maneuvering gene, the gene is randomly assigned any one of the distinct maneuvering strategies that are derived in Chapter 4: Maneuvering Strategies for Maximal Changes in Orbital Geometry. If that maneuver also requires a real-valued angle to resolve any ambiguities with the maneuver, then a random angle normalized between 0 and $2 \pi$ is also assigned. During a small mutation to a maneuvering gene, the maneuvering strategy is left unchanged while the disambiguation angle (if it is required/available for that maneuvering strategy) is randomly perturbed by up to $\frac{\pi}{2}$ in either direction.

Large mutations to a propellant gene result in a random propellant allotment between zero and the maximum available propellant. Small mutations to a propellant gene result in a random perturbation from the current value by up to $5 \%$ of the maximum available propellant. 
Large Mutation

\begin{tabular}{|c|c|c|}
\hline$A_{\text {Before }}$ & & $\mathbf{A}_{\text {After }}$ \\
\hline $\begin{array}{l}\text { Maneuvering Gene } \\
\text { Maneuver \#8 } \\
\text { Disambiguation Angle } 123.4 \mathrm{deg} . \\
\text { Propellant Gene } \\
5.1 \mathrm{~kg} .\end{array}$ & No Mutation & $\begin{array}{l}\frac{\text { Maneuvering Gene }}{\text { Maneuver \#8 }} \\
\text { Disambiguation Angle } 123.4 \mathrm{deg} . \\
\frac{\text { Propellant Gene }}{5.1 \mathrm{~kg} .}\end{array}$ \\
\hline $\begin{array}{l}\text { Maneuvering Gene } \\
\text { Maneuver \#13 } \\
\text { Disambiguation Angle } 321.0 \mathrm{deg} \text {. } \\
\frac{\text { Propellant Gene }}{3.7 \mathrm{~kg} .}\end{array}$ & Large Mutation & $\begin{array}{l}\text { Maneuvering Gene } \\
\text { Maneuver \#6 } \\
\text { Disambiguation Angle } 13.0 \mathrm{deg} . \\
\underline{\text { Propellant Gene }} \\
8.7 \mathrm{~kg} .\end{array}$ \\
\hline $\begin{array}{l}\text { Maneuvering Gene } \\
\text { Maneuver \#1 } 1 \\
\text { Disambiguation Angle N/A } \\
\text { Propellant Gene } \\
9.7 \mathrm{~kg} .\end{array}$ & Large Mutation & $\begin{array}{l}\text { Maneuvering Gene } \\
\text { Maneuver \#5 } \\
\text { Disambiguation Angle } 234.5 \mathrm{deg} \text {. } \\
\text { Propellant Gene } \\
2.8 \mathrm{~kg} .\end{array}$ \\
\hline
\end{tabular}

\section{Small Mutation}

\begin{tabular}{|c|c|c|}
\hline$A_{\text {Before }}$ & & $\mathbf{A}_{\text {After }}$ \\
\hline $\begin{array}{l}\text { Maneuvering Gene } \\
\text { Maneuver \#8 } \\
\text { Disambiguation Angle } 123.4 \mathrm{deg} . \\
\text { Propellant Gene } \\
5.1 \mathrm{~kg} .\end{array}$ & Small Mutation & $\begin{array}{l}\text { Maneuvering Gene } \\
\text { Maneuver \#8 } \\
\text { Disambiguation Angle } 139.3 \mathrm{deg} . \\
\text { Propellant Gene } \\
5.1 \mathrm{~kg} .\end{array}$ \\
\hline $\begin{array}{l}\text { Maneuvering Gene } \\
\text { Maneuver \#13 } \\
\text { Disambiguation Angle } 321.0 \mathrm{deg} . \\
\text { Propellant Gene } \\
3.7 \mathrm{~kg} .\end{array}$ & No Mutation & $\begin{array}{l}\text { Maneuvering Gene } \\
\text { Maneuver \#13 } \\
\text { Disambiguation Angle } 321.0 \text { deg. } \\
\text { Propellant Gene } \\
3.7 \mathrm{~kg} .\end{array}$ \\
\hline $\begin{array}{l}\text { Maneuvering Gene } \\
\text { Maneuver \#1 } \\
\text { Disambiguation Angle N/A } \\
\text { Propellant Gene } \\
9.7 \mathrm{~kg} .\end{array}$ & Small Mutation & $\begin{array}{l}\text { Maneuvering Gene } \\
\text { Maneuver \#1 } 1 \\
\text { Disambiguation Angle N/A. } \\
\text { Propellant Gene } \\
8.9 \mathrm{~kg} .\end{array}$ \\
\hline
\end{tabular}

Figure 43: Depiction of the mutation genetic operator applied to a chromosome used in this work. When a chromosome is mutated, at least one gene within the chromosome is mutated. During a large mutation of a maneuvering gene, the discrete maneuver will be changed as will the disambiguation angle. In contrast, during a small mutation of a maneuvering gene, only the disambiguation angle will be changed. During a large mutation of a propellant gene, the propellant allotment will be changed to some value from zero to the maximum propellant available. 


\section{Guaranteeing Unique Children}

All children are checked to ensure that they are unique from all other children and from all other chromosomes in the parent generation. In view of this, children that are merely clones of their parents will only survive to the next generation if they are unique after undergoing mutation.

\section{Selecting A Subset of a Nondominated Front}

The term "genetic drift" 48 was originally coined by De Jong to describe situations, both in nature and in genetic algorithms, where members of a population tend to exhibit many of the same characteristics. Within the realm of genetic algorithms, Holland ${ }^{54}$ suggested a remedy in the form of a crowding operator which attempted to identify those situations and favor solutions that exhibited more distinct characteristics. Some implementations have focused on identifying the proximity of solutions within the design space/parameters $48,55,56$. The approach taken in this implementation is similar, though not identical, to a feature found in the Multiobjective Genetic Algorithm 49: the proximity of solutions are calculated in objective space rather than parameter space.

When confronted with the need, as deemed necessary in the remainder of this section, to select only a subset of a nondominated front, the algorithm creates a multiobjective sub-problem aimed at obtaining solutions that are as far apart from each other (in objective space) as possible. The pseudo-code is shown in Table 7.

Table 7: Pseudo-code for Selecting A Subset of a Nondominated Front

1. For each solution

\section{For each objective}

3. A separation distance is assigned to the solution equaling the maximum distance (in objective space) to the two nearest neighbors (with respect to the specific objective)

4. A new sub-problem is formed consisting of maximizing the multiobjective separation distances

5. Until the subset population has reached the desired size ...

6. Find those solutions along the nondominated front of the subproblem

7. Remove those solutions from the subproblem

8. Add the original solution to the subset population 
Steps 5-8 obtain "waves" of solutions from the subproblem (see Fig. 44). This is a similar idea employed in the Nondominated Sorting Genetic Algorithm 55 except that it was applied to the original multiobjective problem.

This approach addresses two problems encountered with some of the other algorithms:

(a) There is no need to pre-specify a threshold 49,57 below which two solutions will be "sharing" the same space.

(b) It easily permits the comparison of any pair of fitnesses/objectives that are measured in different units (i.e. incommensurable fitnesses/objectives).

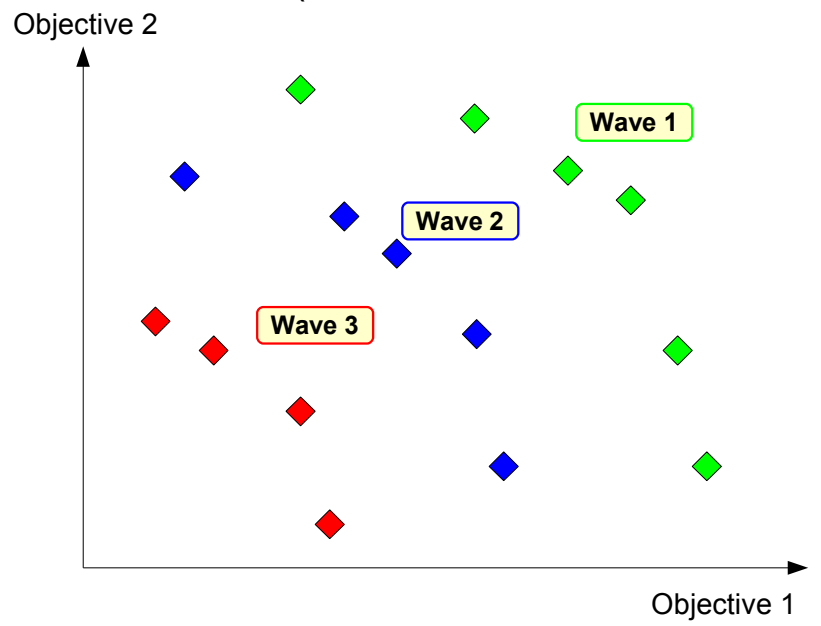

Figure 44: Waves of nondominated solutions to a multiobjective maximization problem are shown.

\section{Partially Populating the Next Generation with Nondominated Solutions}

A portion of the next generation will be filled by the "elite" chromosomes found in the merger of the prior generation and the new children. The number of elite chromosomes that survive ranges between a pre-specified minimum number of slots, elite ${ }_{\min }$, and a pre-specified maximum number of slots, elite ${ }_{\max }$. If the number of nondominated solutions from the merger, $N D_{\text {merge }}$, is less than or equal to elite $_{\min }$, then a two-step process occurs:

1. All of the nondominated merged solutions advance to the next generation

2. The remaining required chromosomes, $N D_{\text {merge }}-$ elite $_{\text {min }}$, are obtained from the merged set using the algorithm described in Section 5.2.3: Selecting A Subset of a Nondominated Front.

If the number of nondominated solutions from the merger, $N D_{\text {merge }}$, is greater than elite $_{\text {min }}$ but less than or equal to elite max $_{\text {ax }}$ then:

- All of the nondominated merged solutions advance to the next generation

If the number of nondominated solutions from the merger, $N D_{\text {merge }}$, is greater than elite $_{\max }$, then: 
- A number of chromosomes equal to elite max $_{\text {is }}$ is obtained from the merged set using the algorithm described in Section 5.2.3: Selecting $A$ Subset of a Nondominated Front.

In short, this step attempts to bring the entire nondominated population over to the next generation as long as it does not exceed elite ${ }_{\max }$. Greater genetic diversity is obtained by not allowing the entire next generation to be composed entirely of the nondominated set.

\section{Partially Populating the Next Generation only with Children}

A portion of the next generation, children, will consist of dominated children.

$$
\text { children }=\min \left(\max \left(\text { elite }_{\min }, N D_{\text {merge }}\right), \text { elite }_{\max }\right)
$$

This assures the introduction of new chromosomes at every generation. The children are selected using the algorithm described in Section 5.2.3: Selecting $A$ Subset of a Nondominated Front.

\section{3: Using a Multiobjective Evolutionary Algorithm to Improve Constellation Coverage}

For any given constellation scenario, the initial population will consist of a random set of chromosomes with the exception of one chromosome that specifies no maneuvering for each satellite. Each chromosome represents one solution to the coverage optimization problem described by Table 5. During the multiobjective evolutionary algorithm, child chromosomes are created at each generation. After creation, the various fitnesses of each chromosome are evaluated. Most of the fitnesses are simply figures-of-merit which require the simulation of the particular scenario (see Chapter 2: Calculating Coverage) of the orbital maneuvering specified for each satellite by each chromosome's genes. Once the fitnesses have been evaluated, the rest of the algorithm proceeds. Generations are repeated until some termination criterion has been satisfied. 


\section{Chapter 6: Examples}

This chapter contains a series of examples that investigate how satellite constellation coverage can be improved by seeking and employing maneuvering strategies that change the orbital geometry of each constellation member.

The Union of Concerned Scientists (UCS) has compiled a dataset of operational satellites as of September 10, 2006 ${ }^{58}$. Appendix A: Summary of Operational Satellites categorizes the UCS data in terms of orbit size, eccentricity, inclination, and propellant mass as a fraction of satellite launch mass. The satellite orbits presented in the following examples are meant to be representative of the low-Earth orbit and medium-Earth orbit satellites contained within that dataset.

In the first example, a single satellite is reconfigured in order to improve the total coverage time of Washington, D.C., USA and Beijing, China over the course of 30 days. This example is designed to investigate the effect of satellite reconfiguration on the coverage of two targets with similar latitudes but with distinctly different longitudes.

In the second example, a single satellite is reconfigured in order to improve total coverage time of Washington, D.C., USA and Montevideo, Uruguay over the course of 30 days. This example is designed to investigate the effect of satellite reconfiguration on the coverage of two targets with similar longitudes but with distinctly different latitudes.

In the third example, three satellites are reconfigured in order to improve total coverage time of Washington, D.C., USA and Montevideo, Uruguay over the course of 30 days. This example is designed to investigate how the individual reconfiguration of multiple satellites improves the coverage provided by the constellation as a whole.

Each example consists of:

- A scenario description

- Settings used during the multi-objective optimization

- Multiobjective results from the optimization

- Analysis of some specific results by using coverage estimation

A scenario consists of a set of Earth targets, a set of available satellites, and a timespan of interest during which coverage is calculated and during which orbital maneuvering is permitted. Each satellite has its own propulsion system and a finite amount of propellant. The propulsion system will be used to employ any one of the maneuvering strategies described in Chapter 4: Maneuvering Strategies for Maximal Changes in Orbital Geometry, as well as a "null" maneuver which performs no maneuvering. Additionally, each satellite is alloted a quantity of propellant to be consumed during the maneuvering. It is assumed 
that the satellites are capable of communicating with/observing targets (i.e. maintaining coverage) while the maneuvering strategies are being executed.

Coverage and figures-of-merit for each of the targets will be calculated as described in Chapter 2: Calculating Coverage. When calculating coverage, only those windows whose durations are of 60 seconds or longer are considered.

Times during which a target is simultaneously covered by more than one satellite are only counted once. Table 4 (Section 4.12: Maneuvering Strategy Thresholds) lists the threshold values used by the maneuvering strategies for all the examples.

The maneuvering strategy for each satellite is assigned/selected by the multiobjective optimization algorithm that seeks to optimize the coverage over both targets in the scenario while also minimizing the constellation's propellant consumption. 


\section{1: Washington, D.C. and Beijing: 30-Day, 1-Satellite}

\subsection{1: Description}

In this scenario, one satellite is maneuvered to improve the total coverage time over Washington, D.C., USA and Beijing, China over the course of 30 days. This example is designed to investigate the effect of satellite reconfiguration on the coverage of two targets with similar latitudes but with distinctly different longitudes.

To address this scenario, the optimization problem seeks to find the nondominated surface that: (1) maximizes the coverage time over Washington, D.C.; (2) maximizes the coverage time over Beijing; and (3) minimizes the propellant consumed by the satellite.

A graphical depiction of the scenario at the initial date is shown in Fig. 45. The evolutionary algorithm settings used for this example are listed in Table 8. The properties for the scenario are summarized in Table 9.

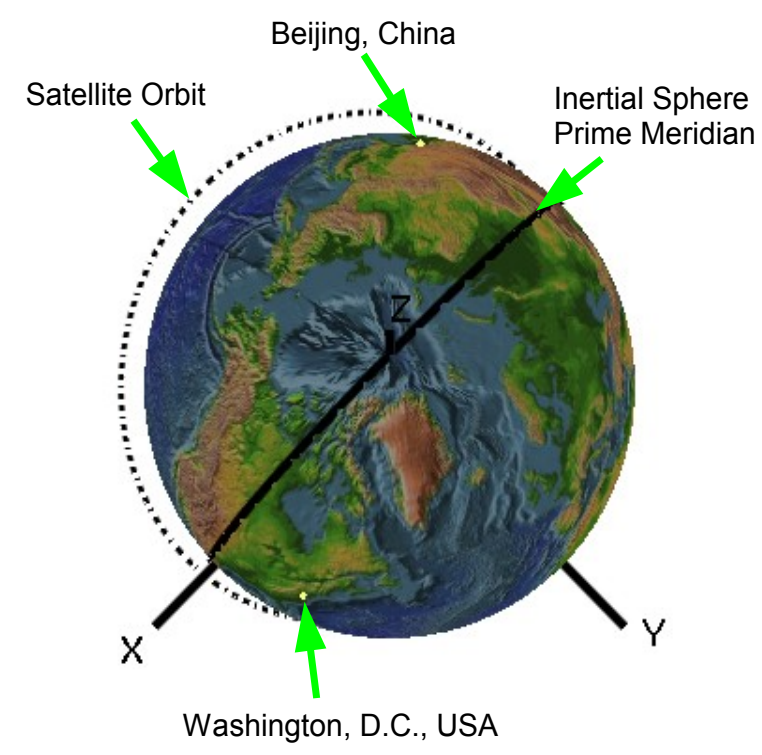

Figure 45: Graphical depiction of the Washington, D.C. and Beijing: 30-Day, 1-

Satellite scenario at the initial date. 
Table 8: Settings used for the multiobjective evolutionary algorithm for Examples 6.1 and 6.2

\begin{tabular}{|c|l|}
\hline Population Size & 30 \\
\hline Offspring Generated per Generation & 24 \\
\hline Maximum Nondominated Elitism & 23 \\
\hline Number of Generations (Termination Criterion) & 20 \\
\hline Crossover Rate & $50 \%$ \\
\hline Small Mutation Rate & $33 \%$ \\
\hline Large Mutation Rate & $17 \%$ \\
\hline
\end{tabular}


Table 9: Scenario properties for Example 6.1

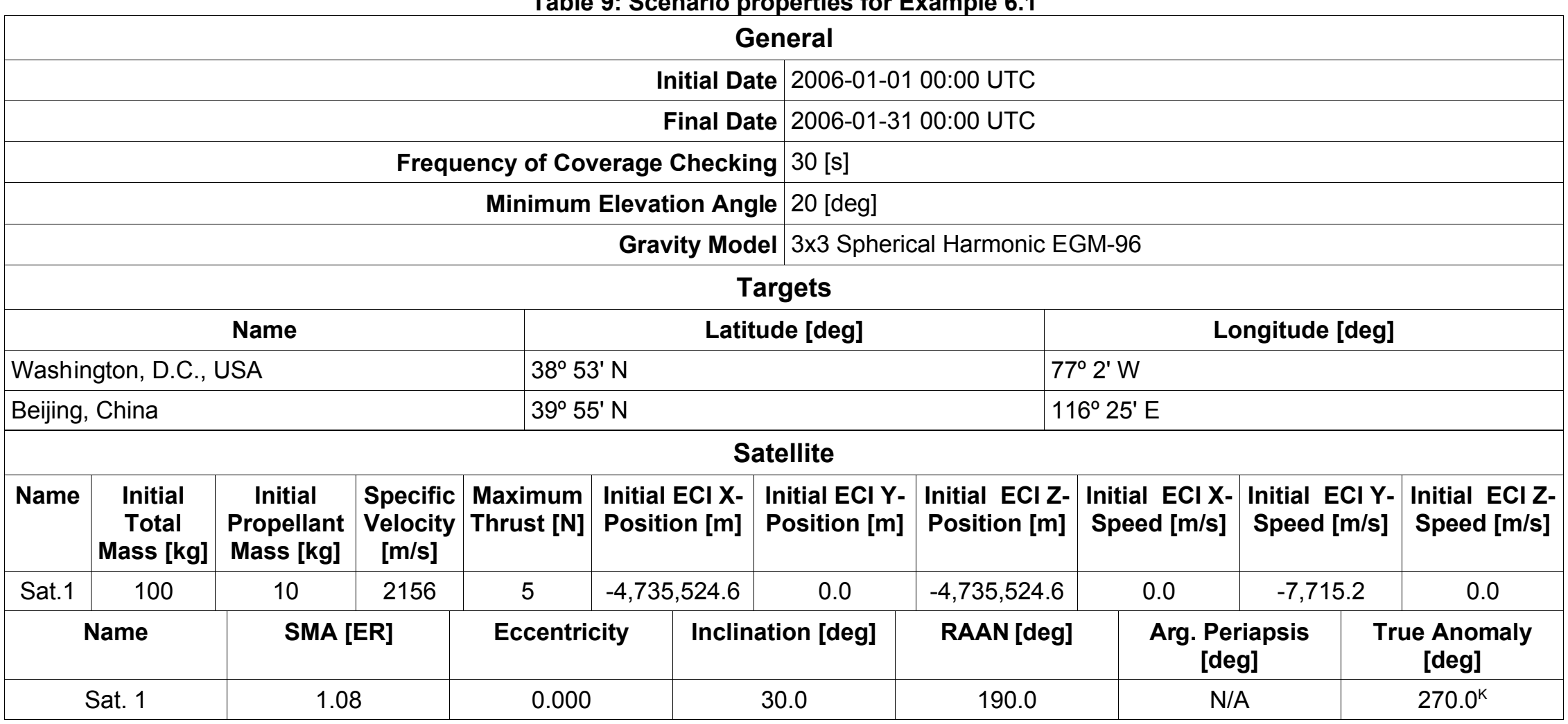

$\mathrm{K}$ Argument of Latitude 


\subsection{2: Results and Analysis}

The nondominated surface from the final generation of the optimization is shown in Fig. 46. The original unoptimized solution is visible in the figure at the point of zero propellant consumption. That solution provides a total coverage time of 0.4 hours for Washington, D.C. and 0.0 hours for Beijing. Notice how the rest of the solutions emanate from that point along a line that extends nearly to maximum propellant consumption (i.e. $10 \mathrm{~kg}$. for the satellite). This line ends at a single point which indicates that the solution which maximizes coverage of Washington, D.C. also maximizes coverage of Beijing. It also indicates that this coverage is obtainable by consuming nearly all of the available propellant (i.e. $9.8 \mathrm{~kg}$.). The final nondominated surface is listed in Table 17 of Appendix C.

The original unoptimized solution is Solution 122 from Table 17. As stated before, it provides a total coverage time of 0.4 hours for Washington, D.C. and 0.0 hours for Beijing. The 30 -day evolution of its visibility map is shown in Fig. 47. Its visibility footprint barely touches Washington, D.C.'s latitude $\left(38^{\circ} 53^{\prime} \mathrm{N}\right)$ and it remains clear of Beijing's latitude $\left(39^{\circ} 55^{\prime} \mathrm{N}\right)$. Over the 30 days, the orbit's ascending node regresses (see Section 3.5.2: Multiple-Day, Single-Satellite Coverage Estimation), but does not further encroach upon either targets' latitudes.

The result which maximizes total coverage time over Washington, D.C. is Solution 1 from Table 17. This solution raises the total coverage time of Washington, D.C. from 0.4 hours to 10.9 hours, and of Beijing from 0.0 hours to 9.7 hours. This solution has the satellite executing the maneuver described in Section 4.4: Maximization of the Rate-of-Change of the Semi-Major Axis while consuming $9.8 \mathrm{~kg}$ out of the available $10 \mathrm{~kg}$. of propellant. The 30-day evolution of the visibility map is shown in Fig. 48. The initial visibility map was described above. By Day 20, the maneuvering has been completed and the orbit has been made eccentric with the apoapsis pointing up towards the Northern Hemisphere. The visibility footprint beneath the apoapsis provides a high-visibility region across the $38^{\text {th }}$ parallel. By Day 30 , second-order gravitational effects have rotated the apsides somewhat (see Section 3.5.2: Multiple-Day, Single-Satellite Coverage Estimation), yet the edge of the coverage footprint is still providing a fair amount of coverage across the target's latitude.

Nearly all of the solutions have the satellite executing either the maneuver described in Section 4.4: Maximization of the Rate-of-Change of the Semi-Major Axis or the maneuver described in Section 4.10: Maximization of the Rate-ofChange of the Semi-Major Axis and Eccentricity while keeping the Argument of Periapsis Constant. The solutions along the nondominated surface differ slightly in their selection of the initial argument of periapsis. However, they primarily distinguish themselves by the fact that greater coverage correlates to consuming more propellant. 


\subsection{3: Summary}

The results from the optimization indicate that the solutions providing the best coverage over both targets are nearly identical. This result is in agreement with Appendix D: Numerical Study of Single-Satellite Coverage Estimation which shows that there is little difference in the coverage provided by a single satellite for targets sharing the same latitude but having different longitudes. Therefore, if the coverage can be improved over one target, then the coverage over other targets sharing the same latitude will also be improved. The results also showed that improving coverage of both targets required consuming more propellant during maneuvering. 


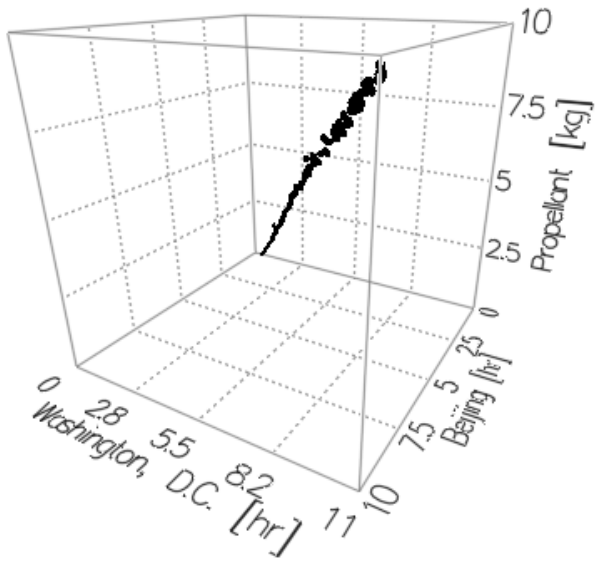

(a)

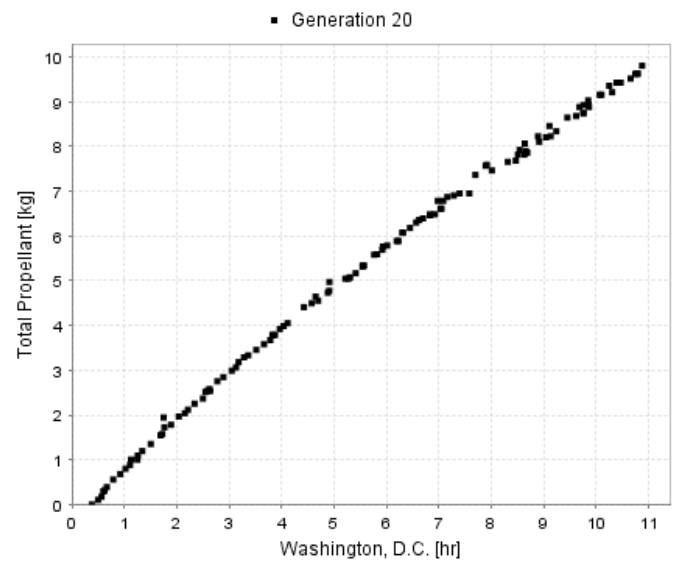

(c)

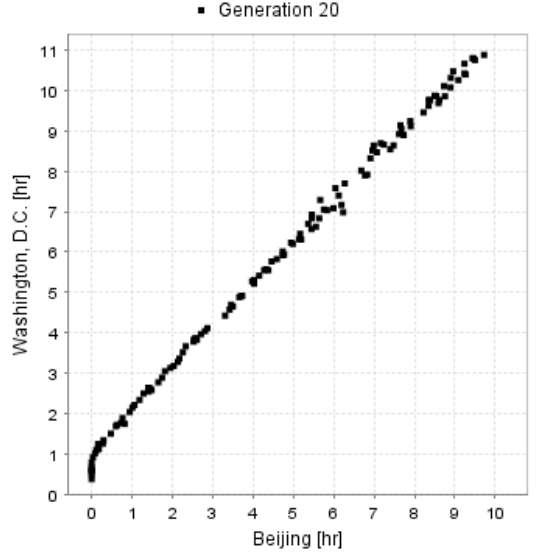

(b)

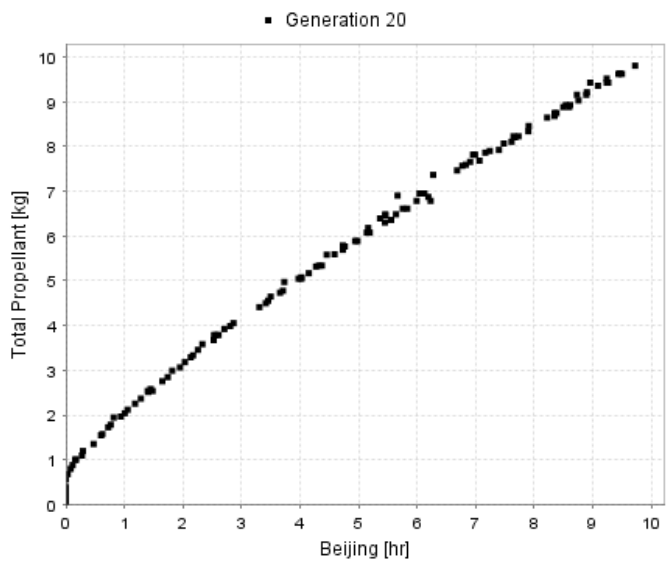

(d)

Figure 46: The final generation of nondominated solutions for Example 6.1 are shown within objective space. Figure (a) shows the solutions in 3D space. Figures (b), (c), and (d) show the 3D solutions projected onto three 2D planes. As visible in the figures, the total coverage time provided by the unoptimized satellite is $\mathbf{0 . 4}$ hours for Washington, D.C. and 0.0 hours for Beijing. 

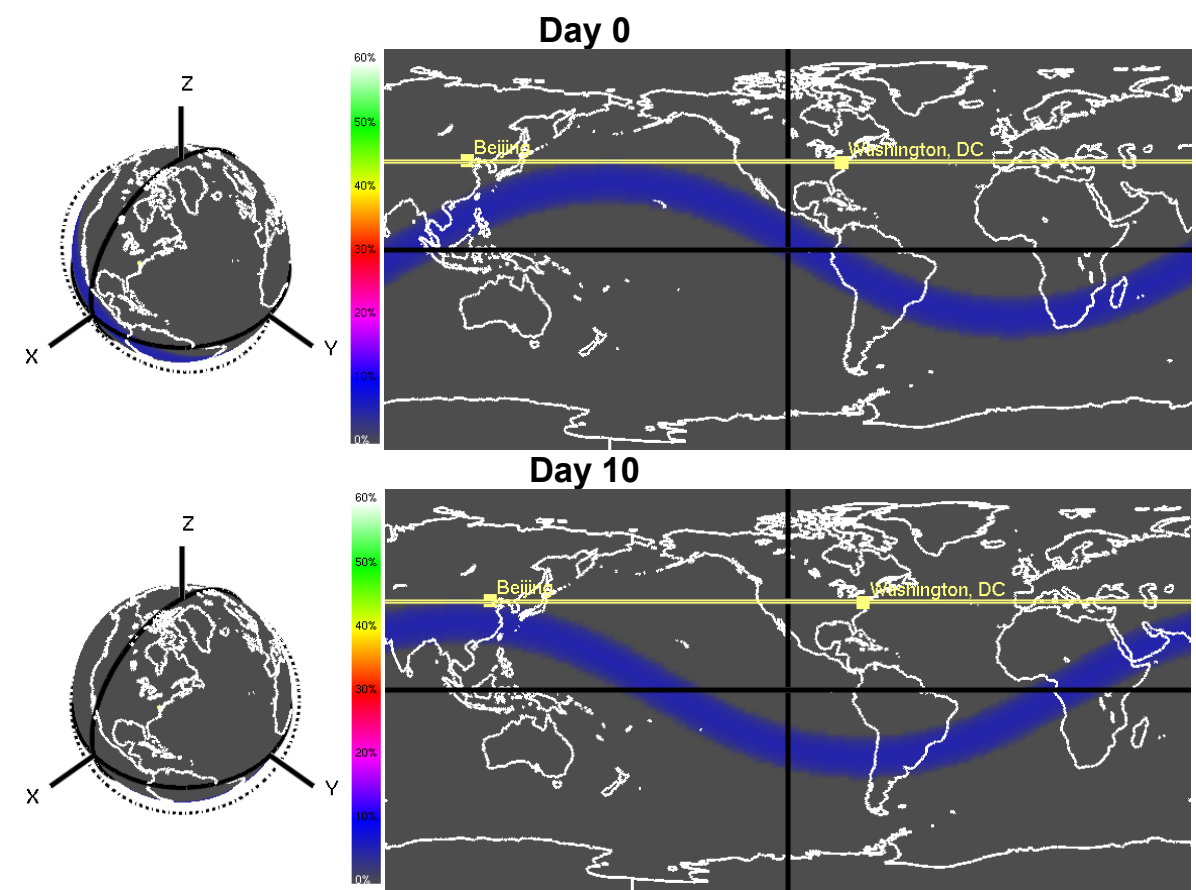

Day 20
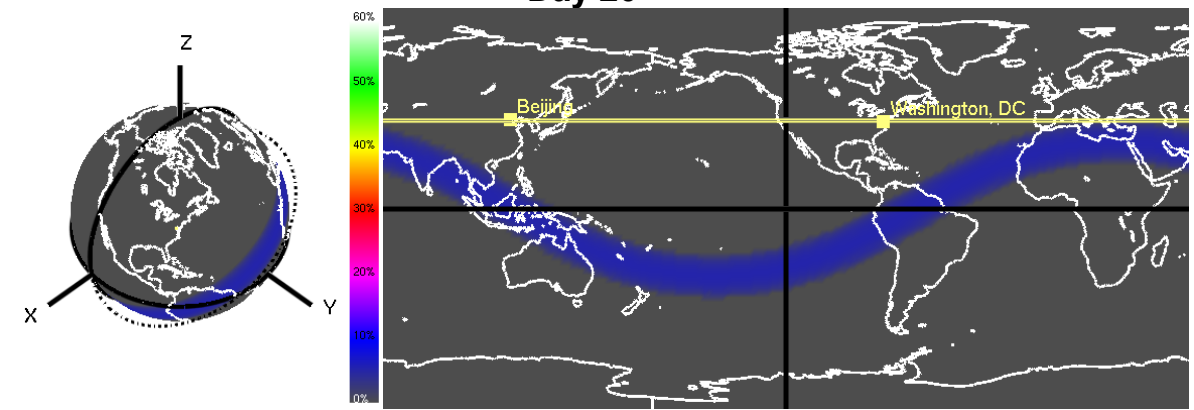

Day 30
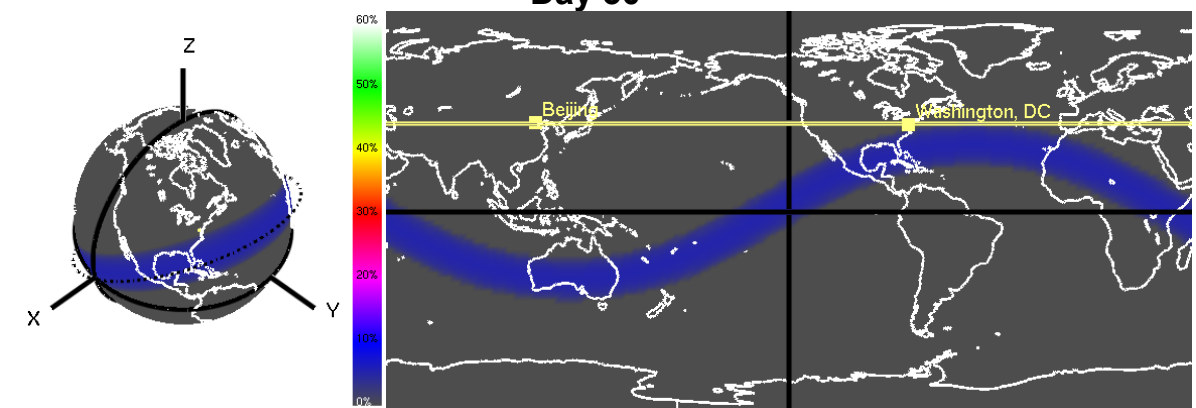

Figure 47: Shown is the 30-day evolution of the visibility map for the original unoptimized solution (Solution 122) of Example 6.1. This solution has the satellite executing no maneuvers, and results in a total coverage of 0.4 hours for Washington, D.C. and of 0.0 hours for Beijing. Washington, D.C., Beijing, and their corresponding latitudes are marked in yellow. The continental outlines are marked in white. Areas in gray are not visible from the satellite orbit. Areas in any other color are visible/covered from the satellite orbit. 

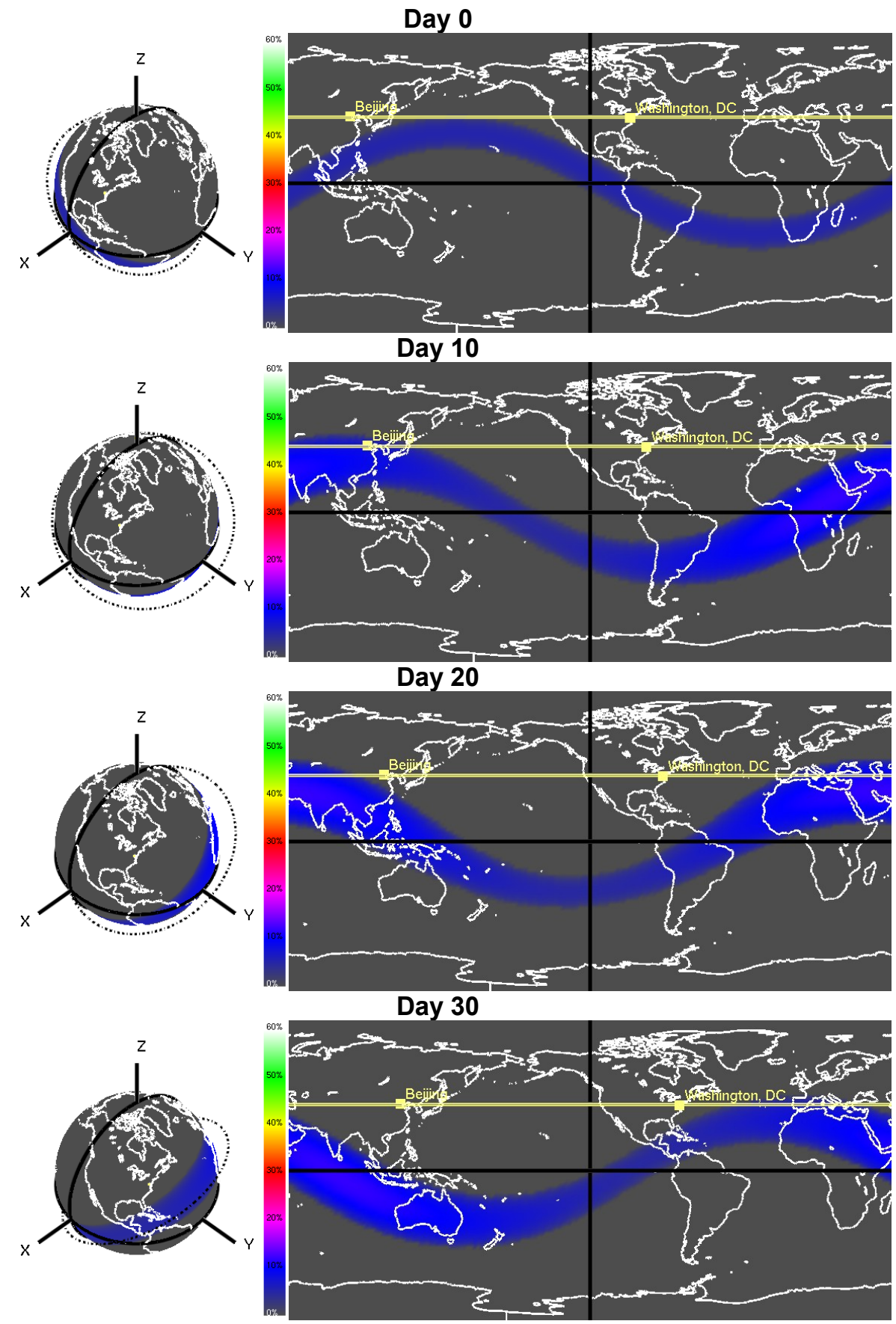

Figure 48: Shown is the 30-day evolution of the visibility map for Solution 1 of Example 6.1. This solution has the satellite executing a maneuver that maximizes coverage over Washington, D.C. (10.9 hours) and Beijing (9.7 hours). (For comparison, the total coverage time provided by the unoptimized satellite is 0.4 hours for Washington, D.C. and 0.0 hours for Beijing.) Washington, D.C., Beijing, and their corresponding latitudes are marked in yellow. The continental outlines are marked in white. Areas in gray are not visible from the satellite orbit. Areas in any other color are visible/covered from the satellite orbit. 


\section{2: Washington, D.C. and Montevideo: 30-Day, 1-Satellite}

\subsection{1: Description}

For this example, one satellite is maneuvered to improve the total coverage time over Washington, D.C., USA and Montevideo, Uruguay over the course of 30 days. This example is designed to investigate the effect of satellite reconfiguration the coverage of two targets with similar longitudes but with distinctly different latitudes.

To address this scenario, the optimization problem seeks to find the nondominated surface that: (1) maximizes the coverage time over Washington, D.C.; (2) maximizes the coverage time over Montevideo; and (3) minimizes the propellant consumed by the satellite.

A graphical depiction of the scenario at the initial date is shown in Fig. 49. The evolutionary algorithm settings used for this example are listed in Table 8. The properties for the scenario are summarized in Table 10.

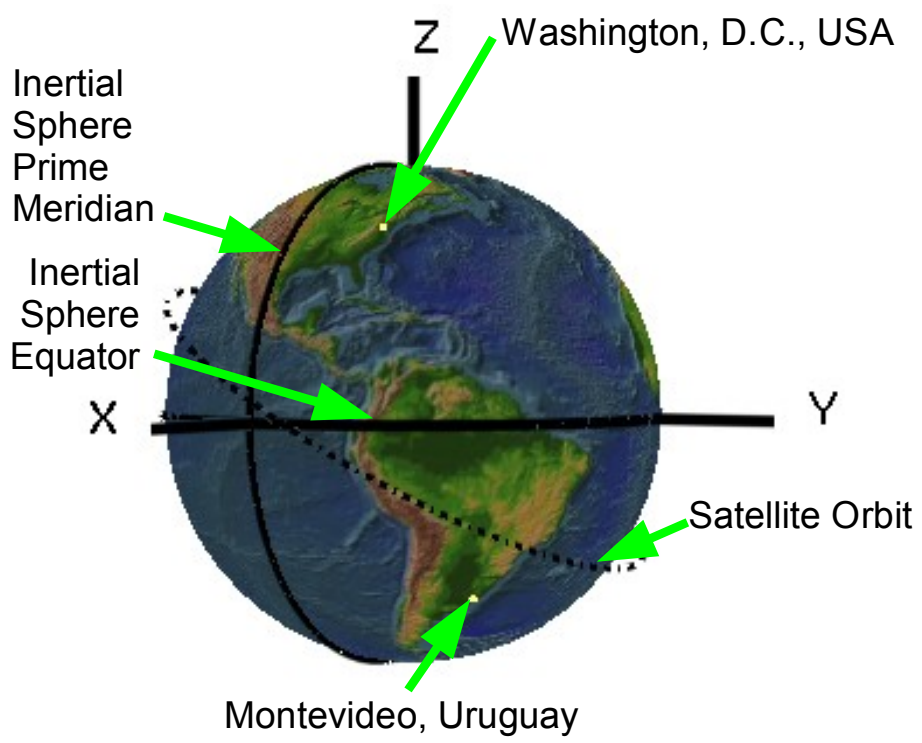

Figure 49: Graphical depiction of the Washington, D.C. and Montevideo: 30-Day, 1-Satellite scenario at the initial date. 
Table 10: Scenario properties for Example 6.2

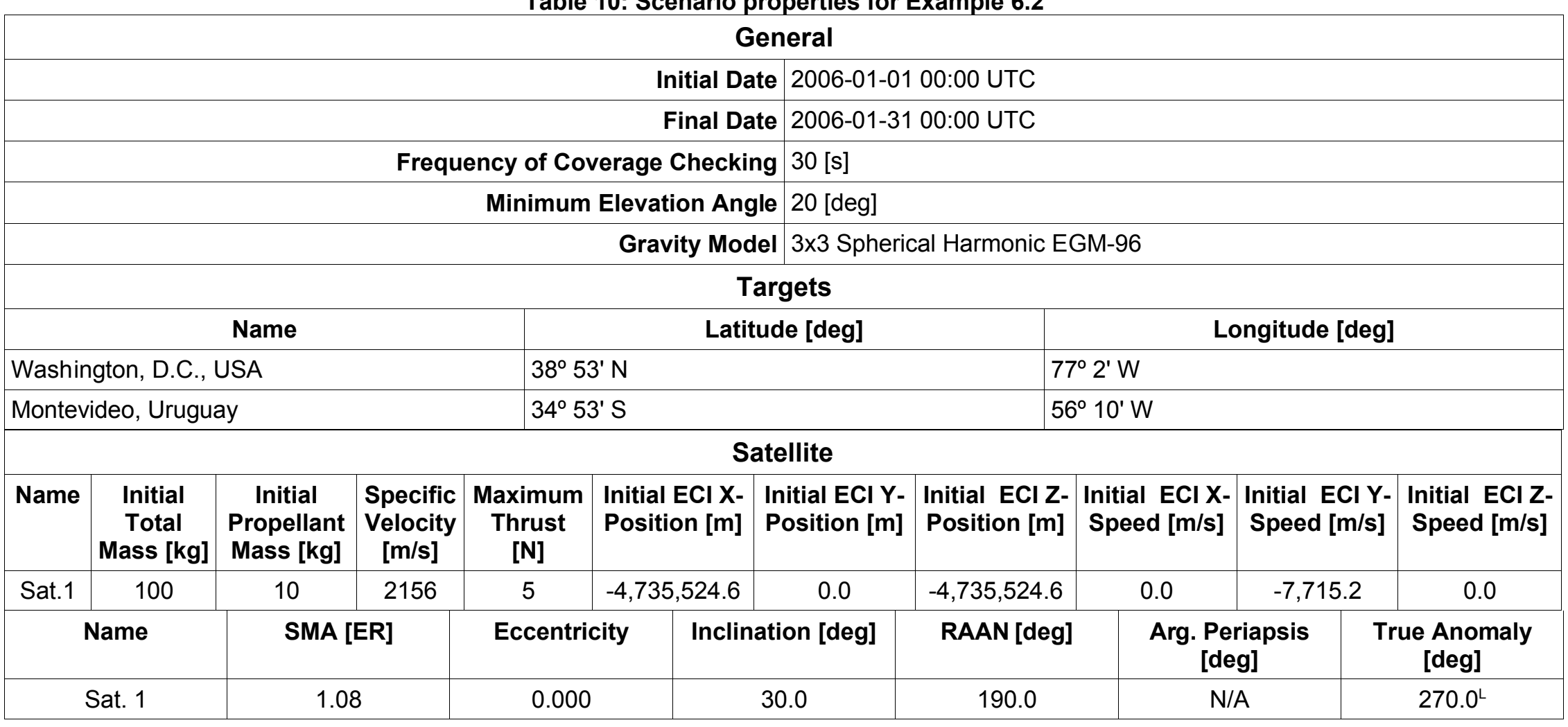

$\mathrm{L}$ Argument of Latitude 


\subsection{2: Results and Analysis}

The nondominated surface from the final generation of the optimization is shown in Fig. 50. The original unoptimized solution is visible in the figure at the point of zero propellant consumption. That solution provides a total coverage time of 0.4 hours for Washington, D.C. and 4.7 hours for Montevideo. Notice how the rest of the solutions emanate from that point along a triangular surface that extends to vertices on the plane representing maximum propellant consumption (i.e. $10 \mathrm{~kg}$. for the satellite). One vertex maximizes coverage of Washington, D.C., and the other vertex maximizes coverage of Montevideo. The final nondominated surface is listed in Table 18 of Appendix C.

The original unoptimized solution is Solution 143 from Table 18. As stated before, it provides a total coverage time of 0.4 hours for Washington, D.C. and 4.7 hours for Montevideo. The 30-day evolution of its visibility map is shown in Fig. 51. Its visibility footprint barely touches Washington, D.C.'s latitude $\left(38^{\circ} 53^{\prime} \mathrm{N}\right)$ although it does cover more of Montevideo's latitude $\left(34^{\circ} 53^{\prime} \mathrm{S}\right)$. Over the 30 days, the orbit's ascending node regresses (see Section 3.5.2: Multiple-Day, Single-Satellite Coverage Estimation), without altering the encroachment across either targets' latitudes.

The result which maximizes total coverage time over Washington, D.C. is Solution 1 from Table 18. This solution raises the total coverage time of Washington, D.C. from 0.4 hours to 11.3 hours, and of Montevideo from 4.7 hours to 8.8 hours. This solution has the satellite executing the maneuver described in Section 4.4: Maximization of the Rate-of-Change of the Semi-Major Axis while consuming all of the available $10 \mathrm{~kg}$. of propellant. The 30-day evolution of the visibility map is shown in Fig. 52 . The initial visibility map was described above. By Day 20, the maneuvering has been completed and the orbit has been made eccentric with the apoapsis pointing up towards the Northern Hemisphere. The visibility footprint beneath the apoapsis provides a high-visibility region across the target's latitude. By Day 30, second-order gravitational effects have rotated the apsides somewhat (see Section 3.5.2: Multiple-Day, Single-Satellite Coverage Estimation), yet the edge of the coverage footprint is still providing a fair amount of coverage across the target's latitude.

The result which maximizes total coverage time over Montevideo is Solution 79 from Table 18. This solution raises the total coverage time of Montevideo from 4.7 hours to 16.3 hours, and of Washington, D.C. from 0.4 hours to 4.0 hours. This solution also has the satellite executing the maneuver described in Section 4.4: Maximization of the Rate-of-Change of the Semi-Major Axis while consuming all of the available $10 \mathrm{~kg}$. of propellant, but with an initial argument of periapsis shifted by nearly 180 degrees. The 30-day evolution of the visibility map is shown in Fig. 53 . To some extent, the visibility map can be described as the opposite of the coverage-maximizing solution for Washington, D.C. (a mirror image across the equator). The greatest difference lies in the amount of coverage overlap across Montevideo's latitude on Day 20. 
This greater overlap is assisted by Montevideo residing at a latitude closer to the equator than does Washington, D.C.

Nearly all of the nondominated solutions consist of executing one of three maneuvers: Maximization of the Rate-of-Change of the Semi-Major Axis (Section 4.4), Maximization of the Rate-of-Change of the Semi-Major Axis and Eccentricity while keeping the Argument of Periapsis Constant (Section 4.10), or Maximization of the Rate-of-Change of the Semi-Major Axis while keeping the Eccentricity constant (Section 4.7). The first two maneuvers make the satellite orbit eccentric. When combined with the proper selection of an initial argument of periapsis, the eccentric orbit's visibility map can favor coverage of one latitude versus another. In comparison, the third maneuver keeps the eccentricity constant, yet due to Montevideo's close proximity to the equator, can create asymmetric coverage conditions.

\subsection{3: Summary}

The results from the optimization indicate that a range of solutions are found to exist that balance the coverage between Washington, D.C. and Montevideo. The range of solutions seem to be continuous in objective space. The results also showed that improving coverage of both targets required consuming more propellant during maneuvering. 


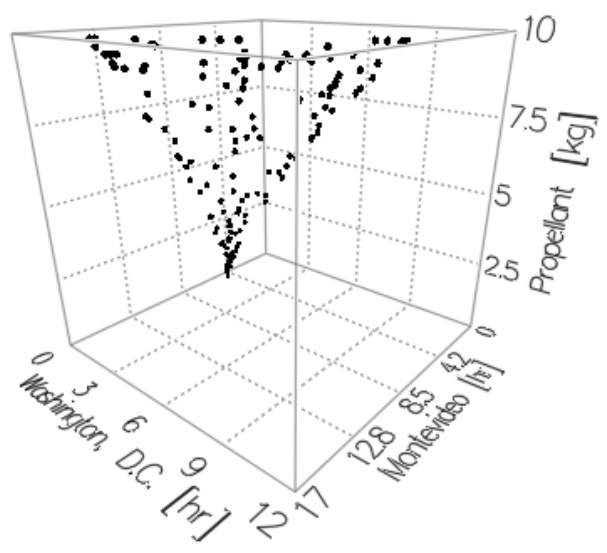

(a)

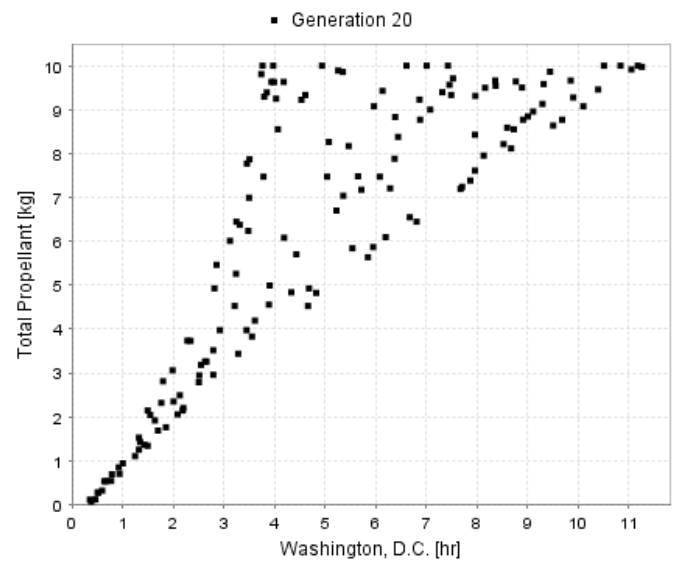

(c)

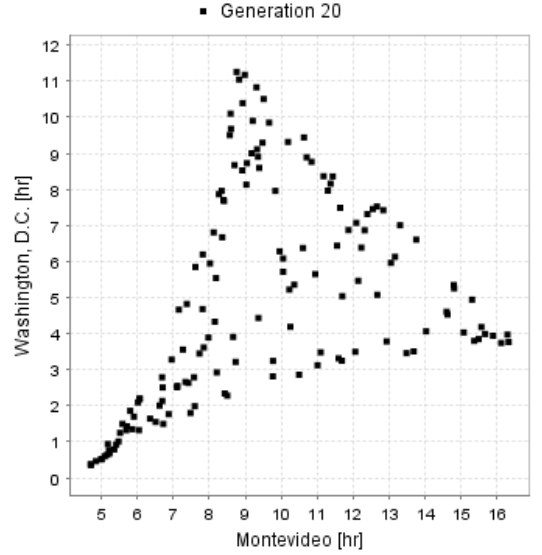

(b)

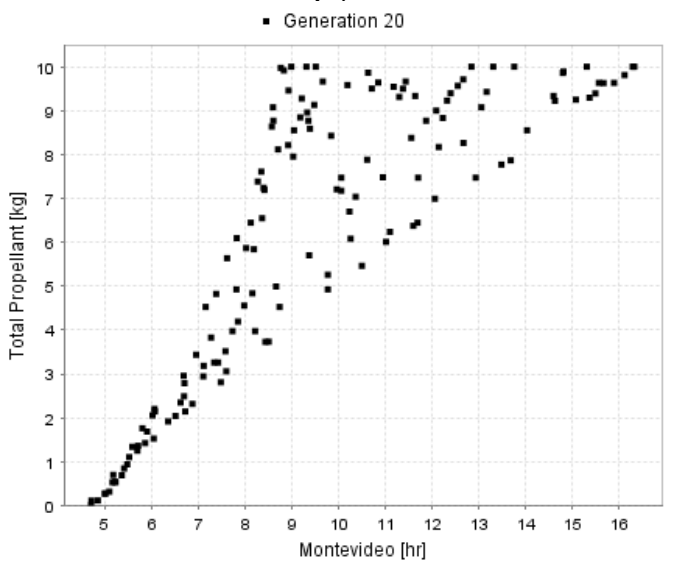

(d)

Figure 50: The final generation of nondominated solutions for Example 6.2 are shown within objective space. Figure (a) shows the solutions in 3D space. Figures (b), (c), and (d) show the 3D solutions projected onto three 2D planes. As visible in the figures, the total coverage time provided by the unoptimized satellite is $\mathbf{0 . 4}$ hours for Washington, D.C. and 4.7 hours for Montevideo. 

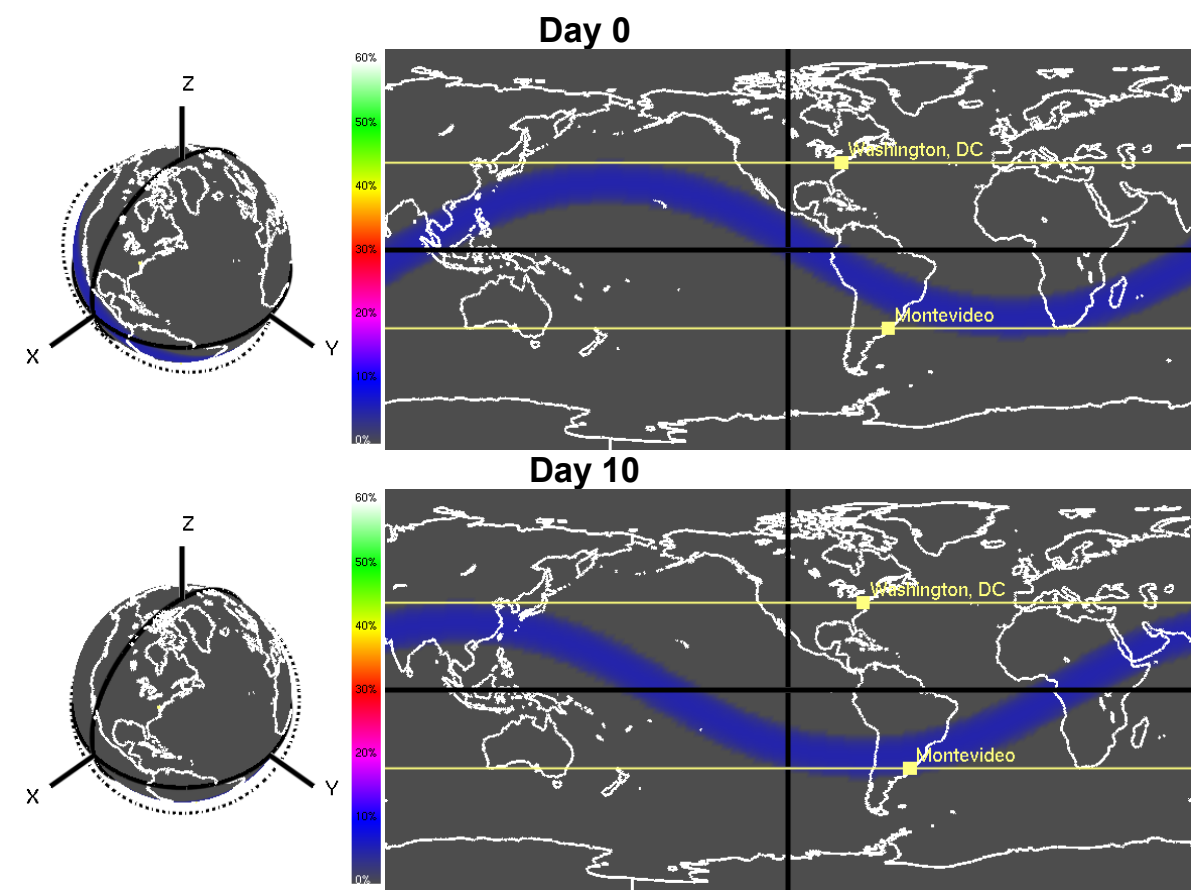

Day 20
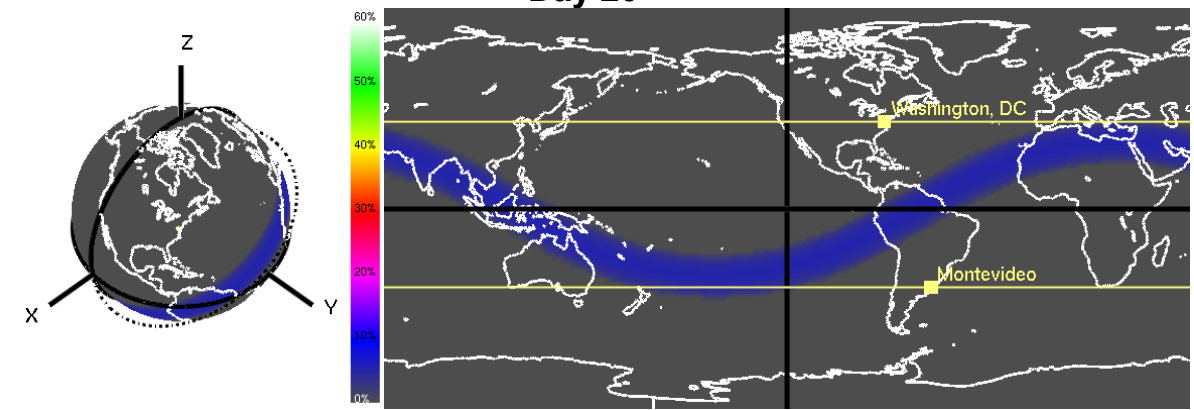

Day 30
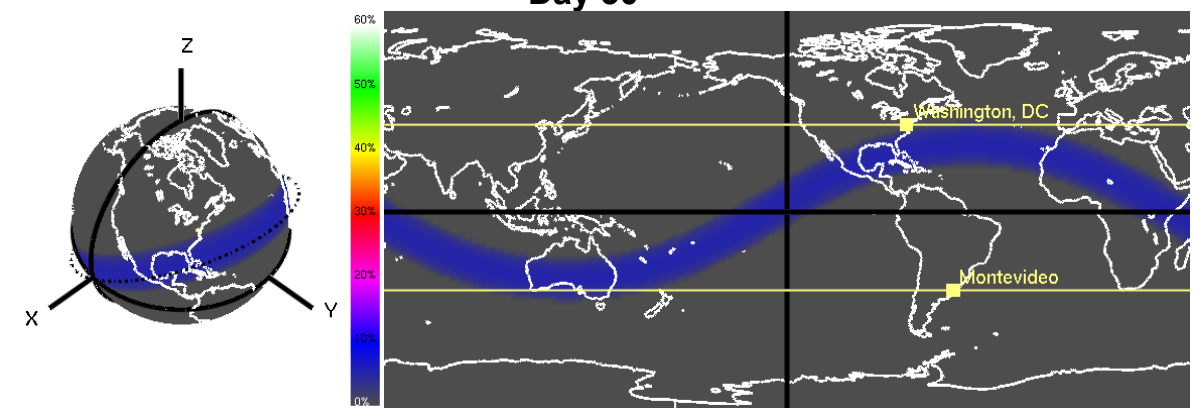

Figure 51: Shown is the 30-day evolution of the visibility map for the original unoptimized solution (Solution 143) of Example 6.2. This solution has the satellite executing no maneuvers, and results in a total coverage of 0.4 hours for Washington, D.C. and of 4.7 hours for Montevideo. Washington, D.C., Montevideo, and their corresponding latitudes are marked in yellow. The continental outlines are marked in white. Areas in gray are not visible from the satellite orbit. Areas in any other color are visible/covered from the satellite orbit. 

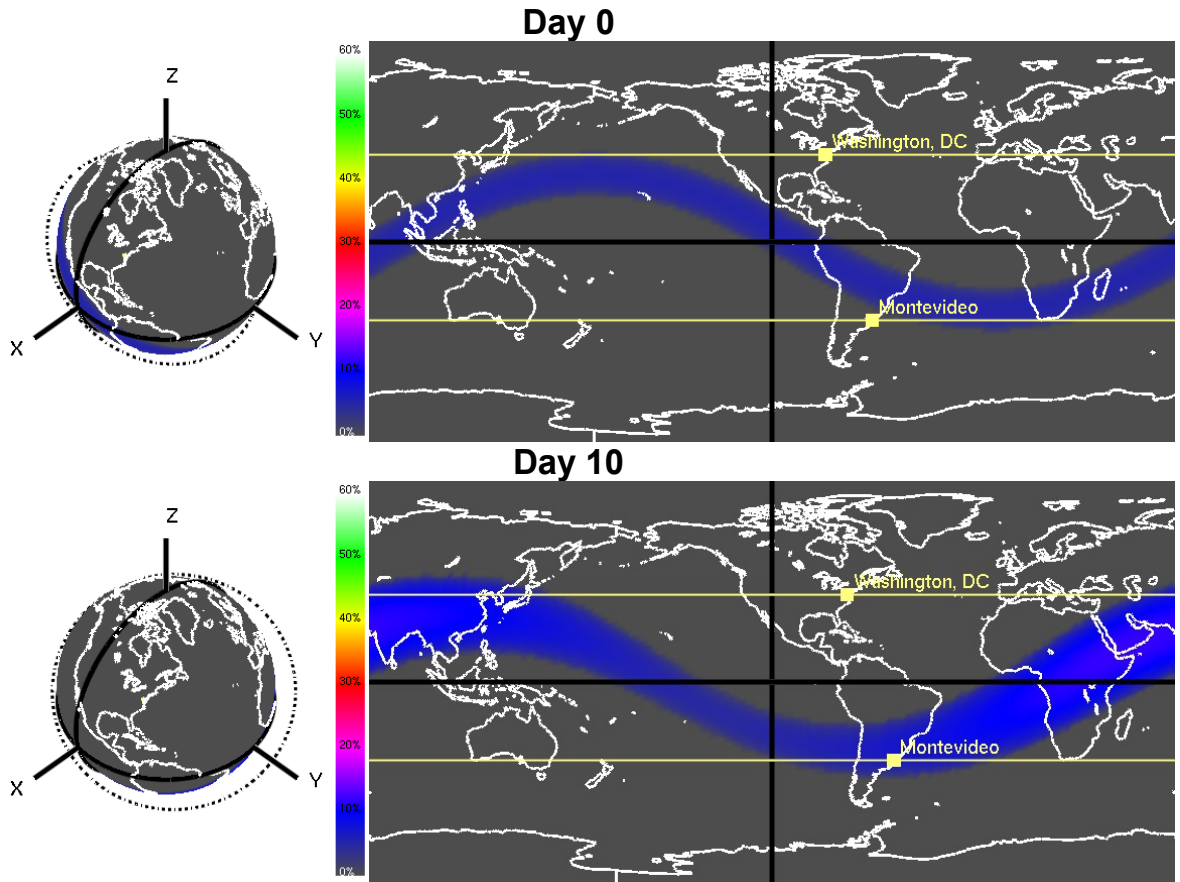

Day 20
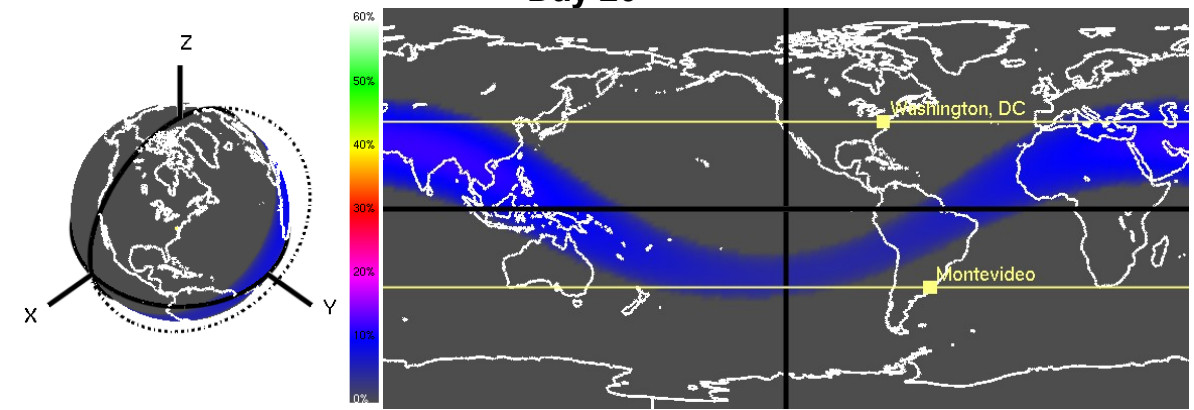

Day 30
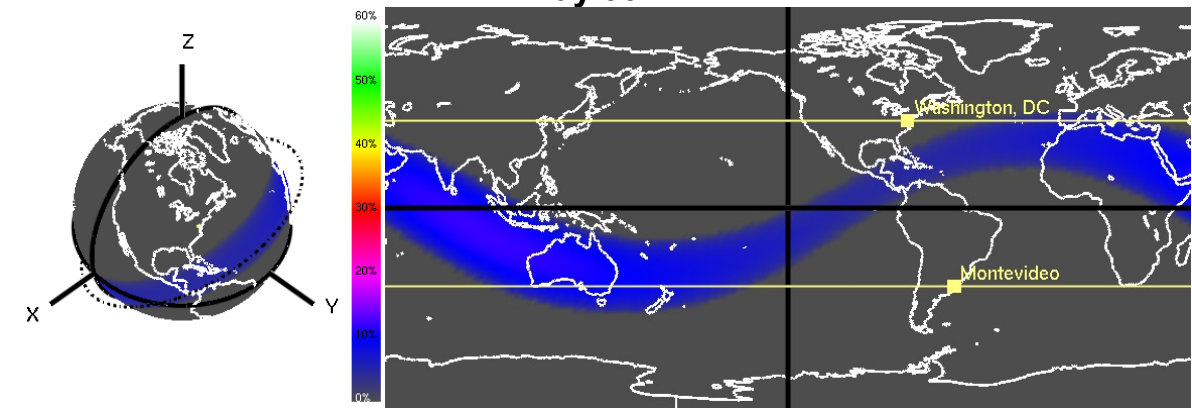

Figure 52: Shown is the 30-day evolution of the visibility map for Solution 1 of Example 6.2. This solution has the satellite executing a maneuver that maximizes coverage over Washington, D.C. of 11.3 hours; Montevideo's coverage is 8.76 hours. (For comparison, the total coverage time provided by the unoptimized satellite is 0.4 hours for Washington, D.C. and 4.7 hours for Montevideo.) Washington, D.C., Montevideo, and their corresponding latitudes are marked in yellow. The continental outlines are marked in white. Areas in gray are not visible from the satellite orbit. Areas in any other color are visible/covered from the satellite orbit. 

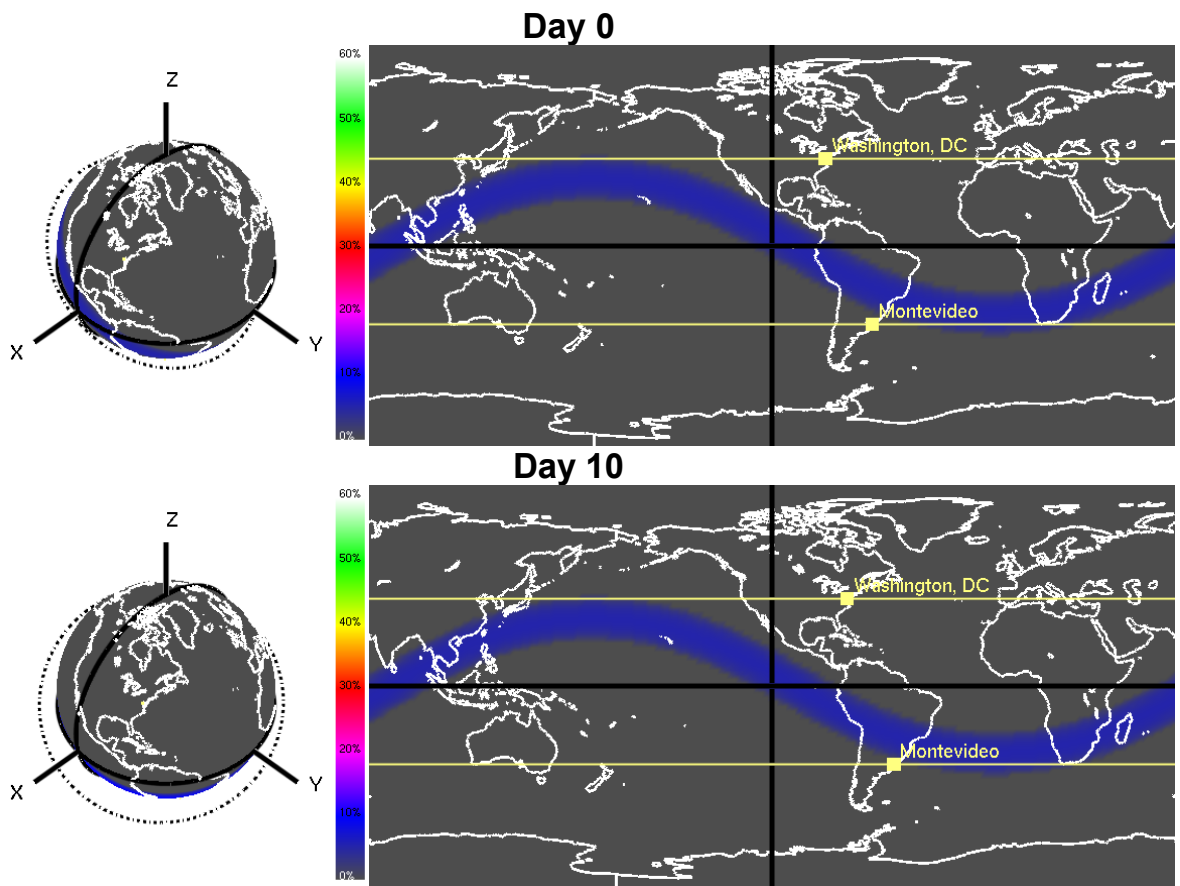

Day 20
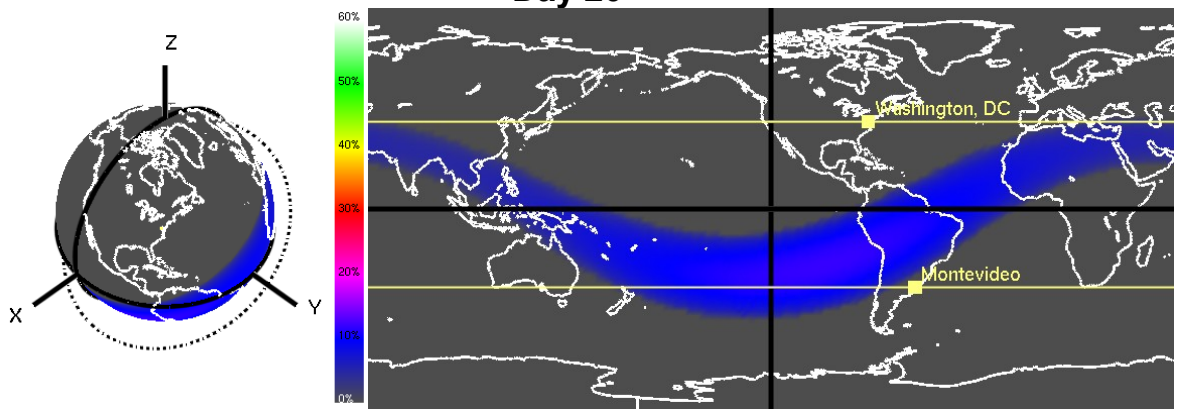

Day 30
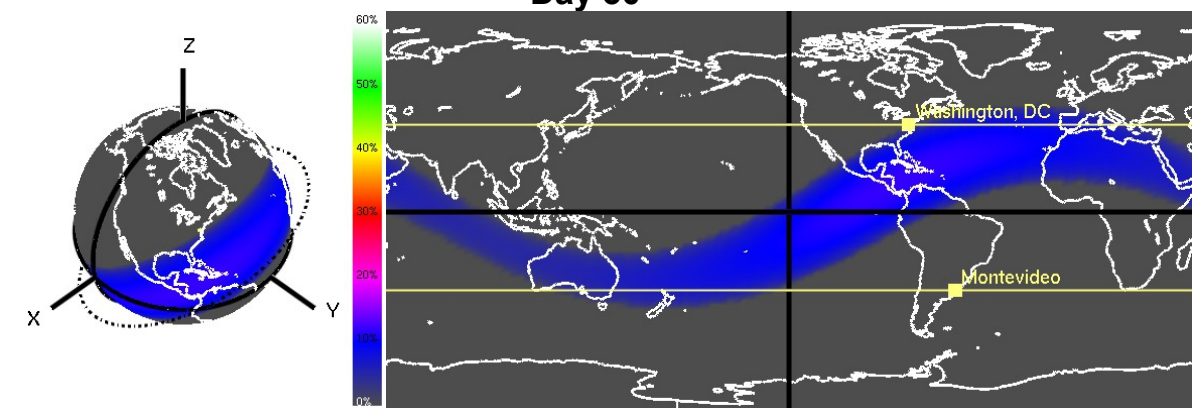

Figure 53: Shown is the 30-day evolution of the visibility map for Solution 79 of Example 6.2. This solution has the satellite executing a maneuver that maximizes coverage over Montevideo of 16.3 hours; Washington, D.C.'s coverage is 4.0 hours. (For comparison, the total coverage time provided by the unoptimized satellite is 0.4 hours for Washington, D.C. and 4.7 hours for Montevideo.) Washington, D.C., Montevideo, and their corresponding latitudes are marked in yellow. The continental outlines are marked in white. Areas in gray are not visible from the satellite orbit. Areas in any other color are visible/covered from the satellite orbit. 


\section{3: Washington, D.C. and Montevideo: 90-Day, 3-Satellites}

\subsection{1: Description}

For this example, three satellites in orbit are maneuvered to improve the total coverage time over Washington, D.C., USA and Montevideo, Uruguay over the course of 90 days. This example is designed to investigate how the individual reconfiguration of multiple satellites improves the coverage provided by the constellation as a whole.

To address this scenario, the optimization problem seeks to find the nondominated surface that: (1) maximizes the coverage time over Washington, D.C.; (2) maximizes the coverage time over Montevideo; and (3) minimizes the propellant consumed by the entire constellation.

A graphical depiction of the scenario at the initial date is shown in Fig. 54. The evolutionary algorithm settings used for this example are listed in Table 11. The properties for the scenario are summarized in Table 12.

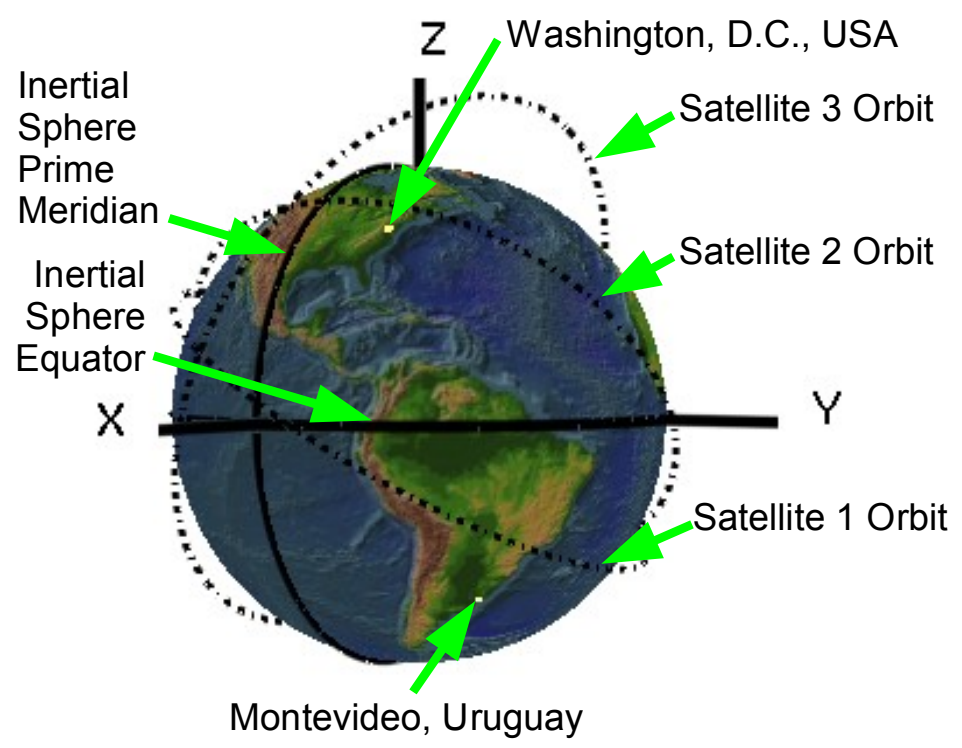

Figure 54: Graphical depiction of the Washington, D.C. and Montevideo: 90-Day, 3-Satellite scenario at the initial date.

Table 11: Settings used for the multiobjective evolutionary algorithm for Example 6.3

\begin{tabular}{|c|l|}
\hline Population Size & 150 \\
\hline Offspring Generated per Generation & 120 \\
\hline Maximum Nondominated Elitism & 113 \\
\hline Number of Generations (Termination Criterion) & 60 \\
\hline Crossover Rate & $50 \%$ \\
\hline Small Mutation Rate & $33 \%$ \\
\hline Large Mutation Rate & $17 \%$ \\
\hline
\end{tabular}


Table 12: Scenario properties for Example 6.3

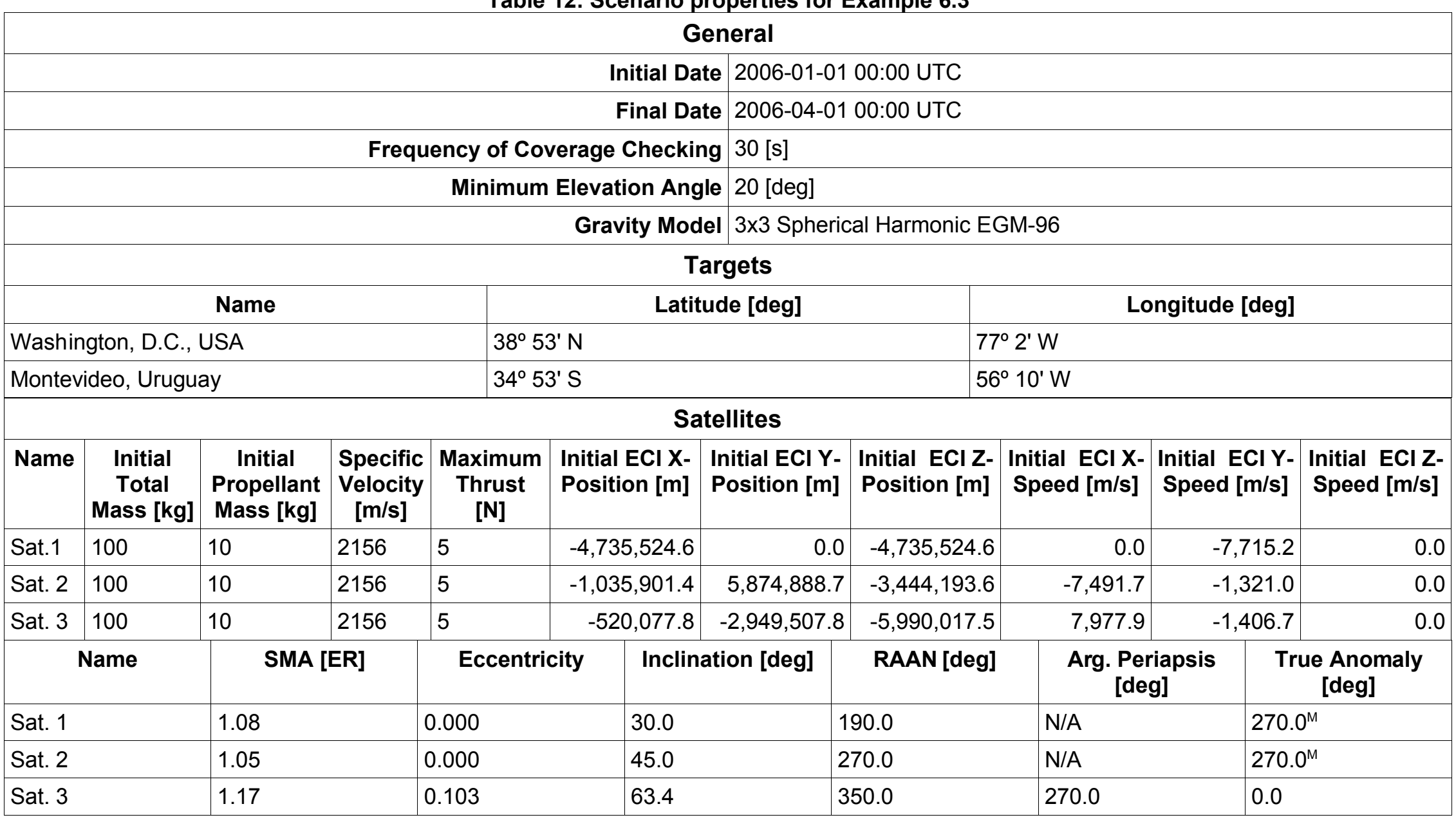

M Argument of Latitude 


\subsection{2: Results and Analysis}

The nondominated surface from the final generation of the optimization is shown in Fig. 55. The original unoptimized solution is visible in the figure at the point of zero propellant consumption. That solution provides a total coverage time of 108.0 hours for Washington, D.C. and 36.0 hours for Montevideo. Two clusters of solutions have been marked on the nondominated surfaces. One end of Cluster 1 is anchored at the original unoptimized solution, while the other end maximizes coverage over Montevideo. Cluster 2 is parallel to Cluster 1 yet extends only from $10 \mathrm{~kg}$. consumption up to the maximum propellant consumption of $30 \mathrm{~kg}$. The final nondominated surface is listed in Table 19 of Appendix C.

The original unoptimized solution is Solution 142 from Table 19. As stated before, it provides a total coverage time of 108.0 hours for Washington, D.C. and 36.0 hours for Montevideo. The 90-day evolution of its visibility map is shown in Fig. 56. The visibility footprint for Satellite 3's eccentric orbit provides two wide swaths of coverage across Washington, D.C.'s latitude $\left(38^{\circ} 53^{\prime} \mathrm{N}\right)$. The visibility footprint also provides two much narrower swaths of coverage across Montevideo's latitude $\left(34^{\circ} 53^{\prime} \mathrm{S}\right)$. Satellite 1 's visibility map barely touches Washington D.C.'s latitude, although it does cover a wider range of Montevideo's latitude. Satellite 2's visibility map covers a large, continuous stretch of Washington D.C.'s latitude, and it completely crosses Montevideo's latitude at two points. Over the 90 days, the three satellite's ascending nodes regress (see Section 3.5.2: Multiple-Day, Single-Satellite Coverage Estimation) without altering the encroachment across either targets' latitudes. The effect of the rotation of the apsides has no effect on Satellite 3's elliptical orbit due to its being critically inclined at 63.4 degrees to cancel such an effect.

The result which maximizes total coverage time over Washington, D.C. is Solution 1 from Table 19. This solution raises the total coverage time of Washington, D.C. from 108.0 hours to 249.8 hours, and of Montevideo from 36.0 hours to 93.2 hours. This solution has all three constellation satellites executing the maneuver described in Section 4.4: Maximization of the Rate-of-Change of the Semi-Major Axis. For Satellites 1 and 2, the orbits are made eccentric with initial angles of periapsis separated by approximately 90 degrees. The solution has the three satellites consuming $28.6 \mathrm{~kg}$ out of the total available propellant of $30 \mathrm{~kg}$. The 90-day evolution of the visibility map is shown in Fig. 57 . The initial visibility map was described above. By Day 30 , all of the maneuvering has been completed, and the constellation's visibility footprints cover nearly all of Washington D.C.'s latitude. The increase in Satellite 3's visibility footprint is significant in both its width across the latitude as well as in the increase of its coverage time over the footprint (denoted by the more intense color). Over the remaining 60 days, the visibility maps beneath the apoapsides of Satellites 1 and 2 alternate in providing coverage adjacent to Satellite 3's visibility footprint. This alternation is caused by the second-order gravity effects, but the appropriate 
relative phasing has been identified by the multiobjective evolutionary algorithm to provide improved coverage over the 90 days.

The result which maximizes total coverage time over Montevideo is Solution 36 from Table 19. This solution raises the total coverage time of Washington, D.C. from 108.0 hours to 203.8 hours, and of Montevideo from 36.0 hours to 117.2 hours. This solution has Satellite 1 executing the maneuver described in Section 4.4: Maximization of the Rate-of-Change of the Semi-Major Axis, Satellite 2 executing the maneuver described in Section 4.10: Maximization of the Rate-of-Change of the Semi-Major Axis and Eccentricity while keeping the Argument of Periapsis Constant, and Satellite 3 executing the maneuver described in Section 4.7: Maximization of the Rate-of-Change of the Semi-Major Axis while keeping the Eccentricity constant. The solution has the three satellites consuming $29.8 \mathrm{~kg}$ out of the total available propellant of $30 \mathrm{~kg}$. The 90-day evolution of the visibility map is shown in Fig. 58. The initial visibility map was described above. By Day 30, all of the maneuvering has been completed, and the constellation's visibility footprints cover most of Montevideo's latitude. Satellite 3's maneuver causes its two pre-existing swaths of coverage to increase in longitudinal width. Over the remaining 60 days, the visibility maps of Satellites 1 and 2 alternate in providing coverage adjacent to Satellite 3's visibility legs, similar to what occurred for the coverage-maximizing solution for Washington D.C.

Of particular interest are the two clusters of solutions that favor one target over another for any given amount of propellant consumption. Cluster 1 solutions are the only members of the nondominated surface capable of providing coverage of Washington, D.C. for propellant consumptions less than approximately $10 \mathrm{~kg}$. However, above $10 \mathrm{~kg}$, Cluster 2 solutions are capable of providing more coverage of Washington D.C. for the same amount of propellant. Similarly, above $10 \mathrm{~kg}$, the Cluster 1 solutions are capable of providing more coverage of Montevideo for the same amount of propellant.

Although there are many solutions in Cluster 1 , most of the solutions are characterized by the following:

- between $0 \mathrm{~kg}$ and $10 \mathrm{~kg}$. of propellant consumption

- Satellite 1 does not maneuver.

- between $24 \mathrm{~kg}$ and $30 \mathrm{~kg}$. of propellant consumption

- Satellite 2 executes Maximization of the Rate-of-Change of the SemiMajor Axis while using nearly all of the available propellant.

- Satellite 3 executes Maximization of the Rate-of-Change of the SemiMajor Axis while keeping the Eccentricity constant while using nearly all of the available propellant.

- Satellite 1 also executes either Maximization of the Rate-of-Change of the Semi-Major Axis or Maximization of the Rate-of-Change of the Semi-Major Axis and Eccentricity while keeping the Argument of 
Periapsis Constant but with propellant consumptions that increase with coverage.

Although there are many solutions in Cluster 2, most of the solutions are characterized by the following:

- between $10 \mathrm{~kg}$ and $20 \mathrm{~kg}$. of propellant consumption

- Satellite 1 does not maneuver.

- Satellite 3 executes Maximization of the Rate-of-Change of the SemiMajor Axis while using nearly all of the available propellant.

- Satellite 2 also executes Maximization of the Rate-of-Change of the Semi-Major Axis but with propellant consumptions that increase with coverage.

- between $20 \mathrm{~kg}$ and $30 \mathrm{~kg}$. of propellant consumption

- Satellites 2 and 3 executes Maximization of the Rate-of-Change of the Semi-Major Axis while using nearly all of the available propellant.

- Satellite 1 executes either Maximization of the Rate-of-Change of the Semi-Major Axis or Maximization of the Rate-of-Change of the SemiMajor Axis and Eccentricity while keeping the Argument of Periapsis Constant but with propellant consumptions that increase with coverage.

\subsection{3: Summary}

The results from the optimization indicate that two clusters of solutions are found to exist that favor either Washington D.C. (Cluster 2) or Montevideo (Cluster 1). Each of the clusters contains a set of maneuvering strategies that are somewhat distinct from the other. However, they both share the correlation that the greatest coverage over either target is only attainable by consuming all of the available propellant.

An interesting characteristic of the solutions is that the maneuvers are oriented in such a way as to contend/take advantage of the second-order gravity effects (described in Section 3.5.2: Multiple-Day, Single-Satellite Coverage Estimation). The coverage-maximizing maneuvering strategies tend to drive the initially circular orbits (Satellites 1 and 2) to becoming elliptical. However, these strategies do not necessarily orient the apoapsides immediately over a target's latitude. Rather, the apoapsides are initially oriented such that the second-order gravitational effects cause the underlying high-visibility regions to encroach, linger, and retreat from a target's latitude in a coordinated manner. 


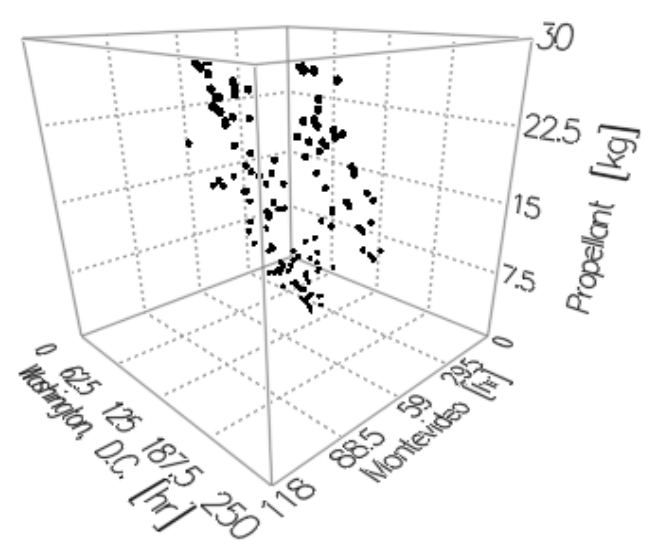

(a)

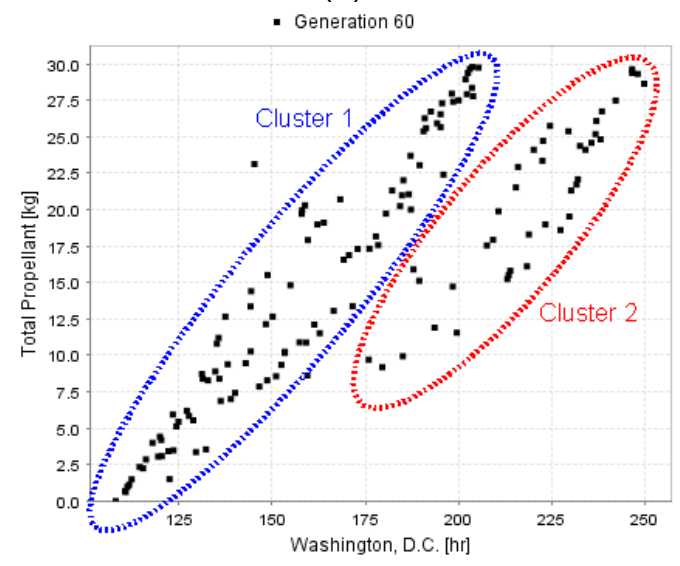

(c)

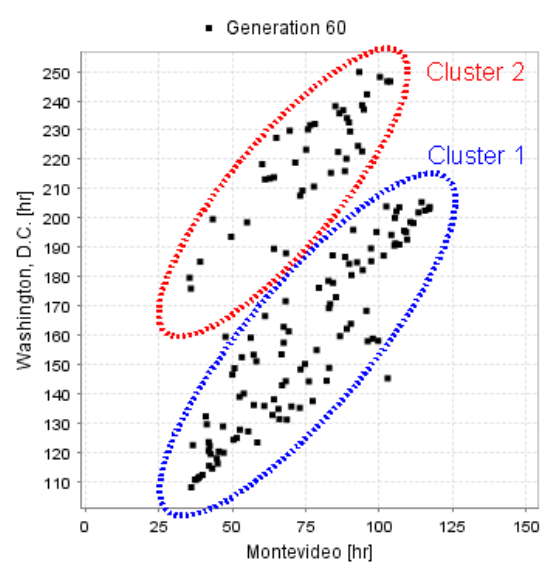

(b)

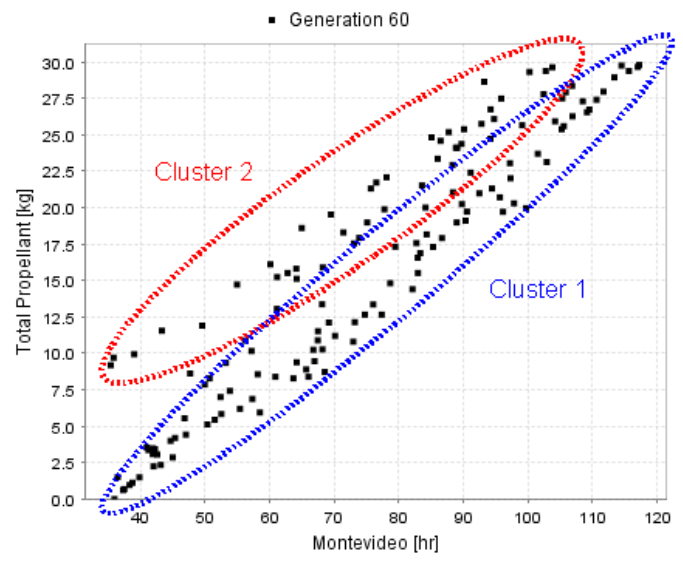

(d)

Figure 55: The final generation of nondominated solutions for Example 6.3 are shown within objective space. Figure (a) shows the solutions in 3D space. Figures (b), (c), and (d) show the 3D solutions projected onto three 2D planes. As visible in the figures, the total coverage time provided by the unoptimized constellation is 108.0 hours for Washington, D.C. and 36.0 hours for Montevideo. Two sets of solutions (Clusters 1 and 2) have been circled each of which reach different areas of the objective space. 

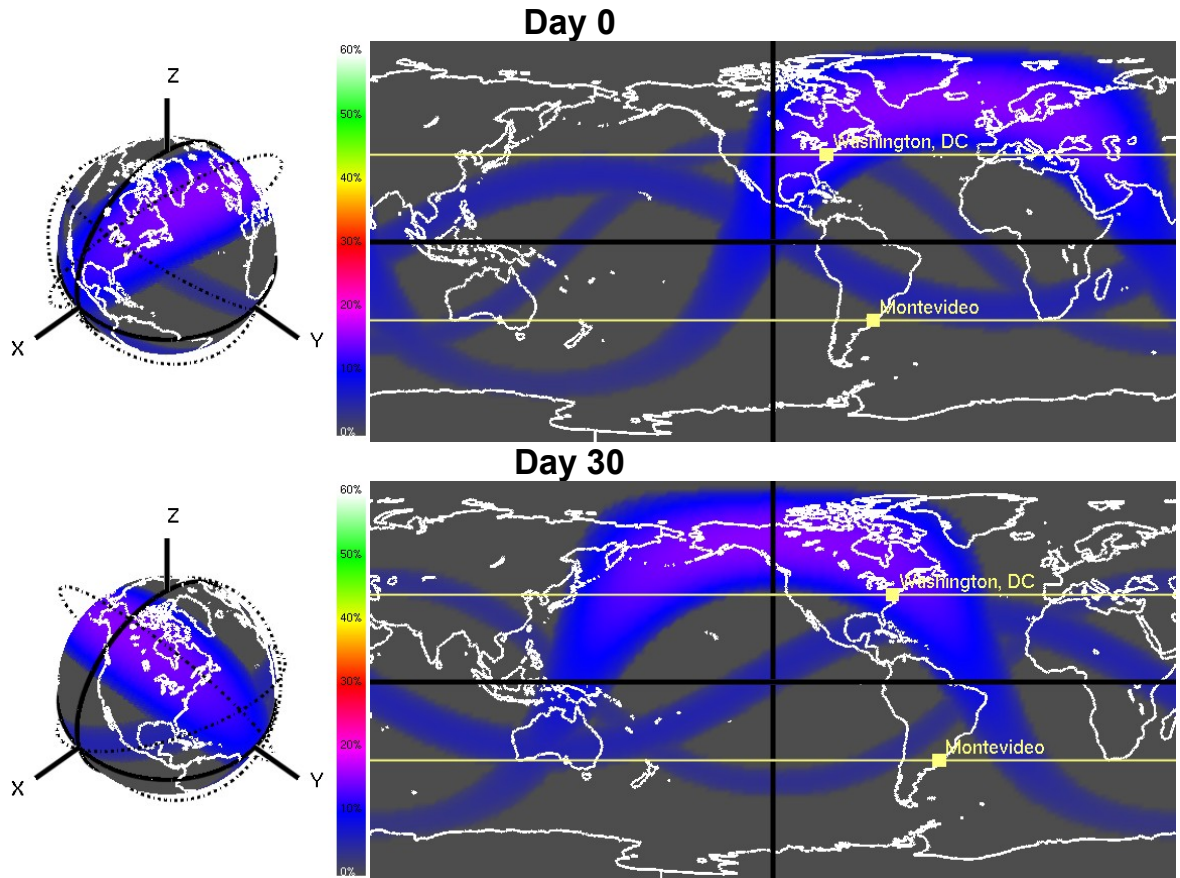

Day 60
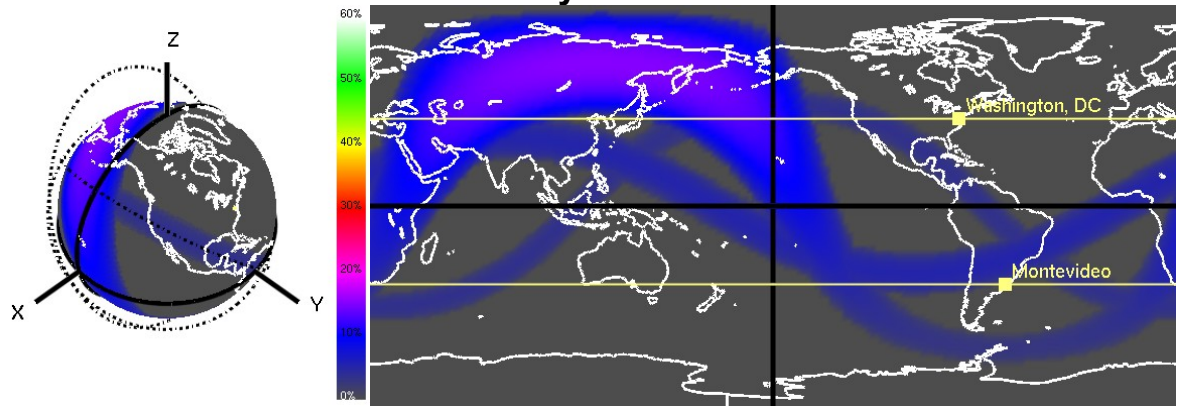

\section{Day 90}
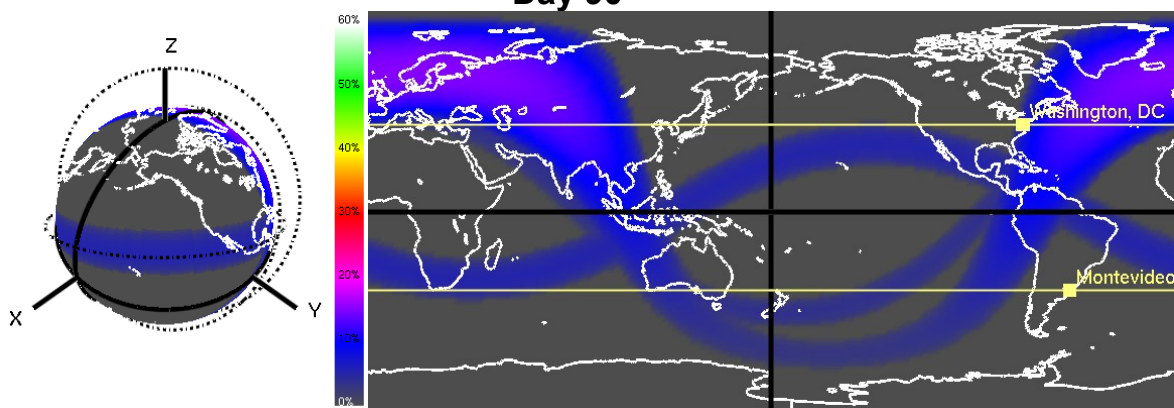

Figure 56: Shown is the 90-day evolution of the visibility map for the original unoptimized solution (Solution 142) of Example 6.3. This solution has the satellite executing no maneuvers, and results in a total coverage of 108.0 hours for Washington, D.C. and 36.0 hours for Montevideo. Washington, D.C., Montevideo, and their corresponding latitudes are marked in yellow. The continental outlines are marked in white. Areas in gray are not visible from the satellite orbit. Areas in any other color are visible/covered from the satellite orbit. 

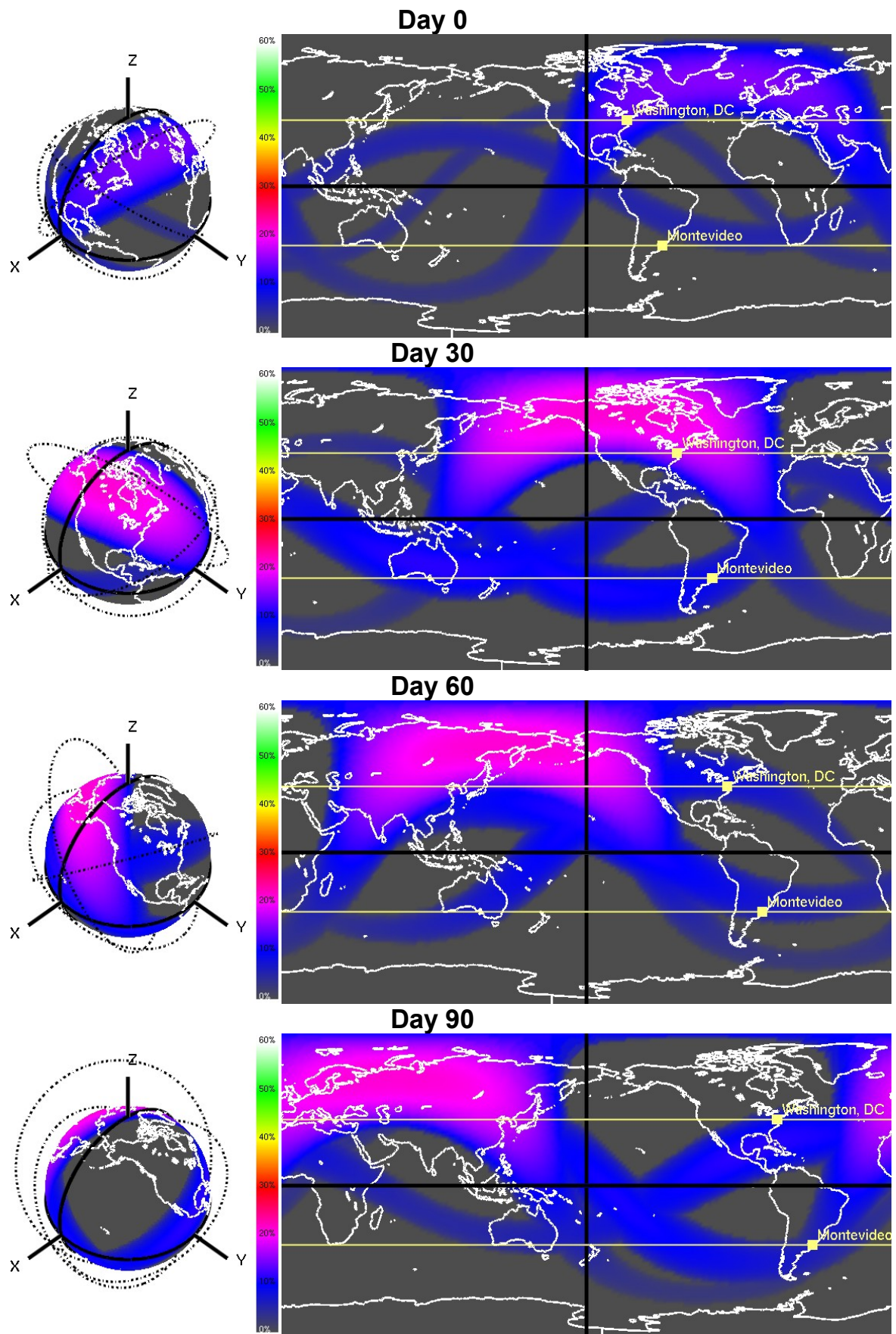

Figure 57: Shown is the 90-day evolution of the visibility map for Solution 1 of Example 6.3. This solution has the satellite executing a maneuver that maximizes coverage over Washington, D.C. of 249.8 hours; Montevideo's coverage is 93.2 hours. (For comparison, the total coverage time provided by the unoptimized satellite is 108.0 hours for Washington, D.C. and 36.0 hours for Montevideo.) Washington, D.C., Montevideo, and their corresponding latitudes are marked in yellow. The continental outlines are marked in white. Areas in gray are not visible from the satellite orbit. Areas in any other color are visible/covered from the satellite orbit. 

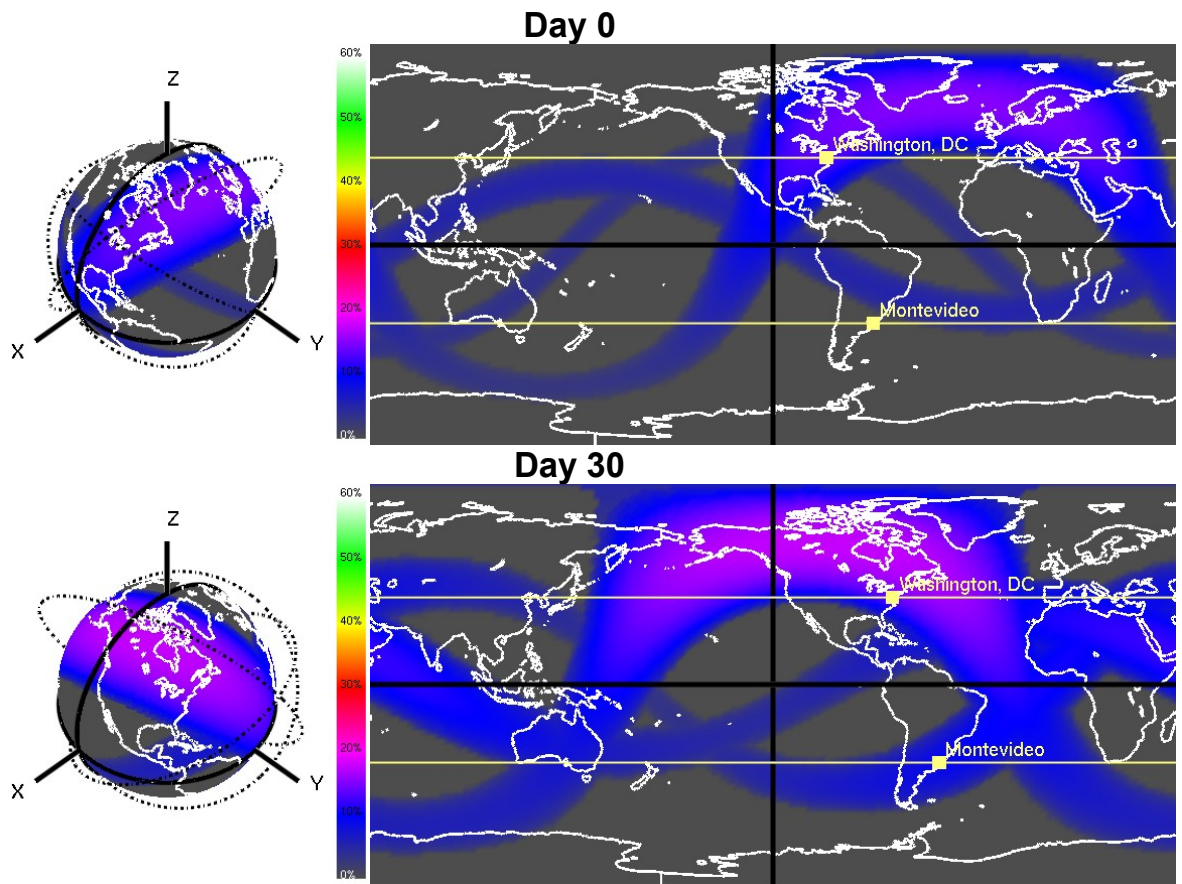

Day 60
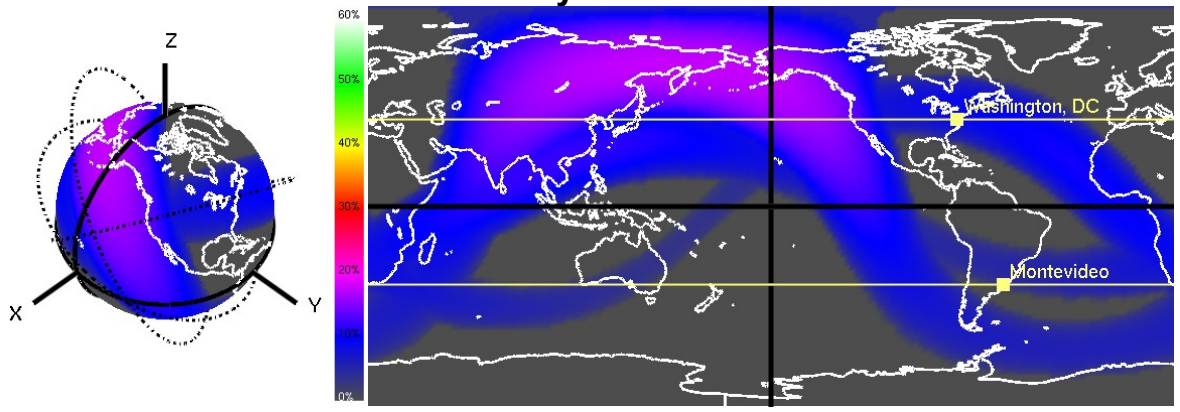

\section{Day 90}
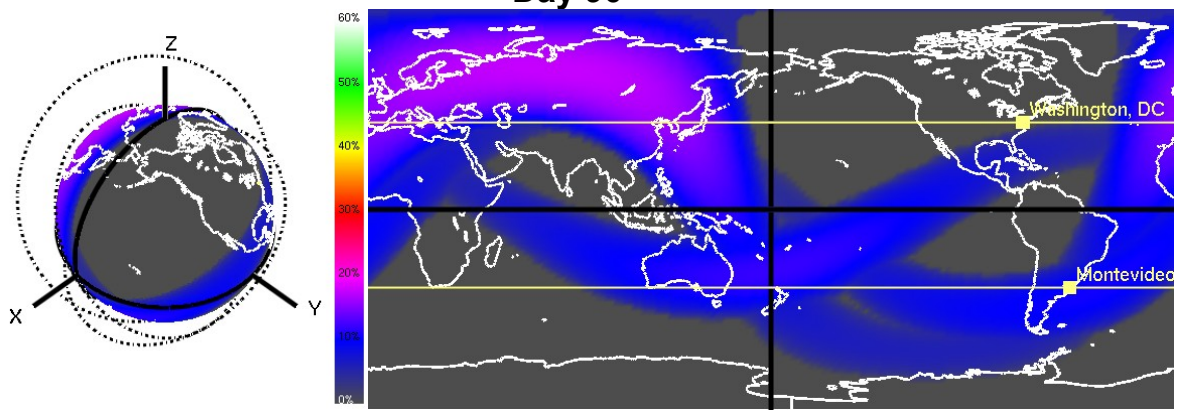

Figure 58: Shown is the 90-day evolution of the visibility map for Solution $\mathbf{3 6}$ of Example 6.3. This solution has the satellite executing a maneuver that maximizes coverage over Montevideo of 117.2 hours; Washington D.C.'s coverage is 203.8 hours. (For comparison, the total coverage time provided by the unoptimized satellite is 108.0 hours for Washington, D.C. and $\mathbf{3 6 . 0}$ hours for Montevideo.) Washington, D.C., Montevideo, and their corresponding latitudes are marked in yellow. The continental outlines are marked in white. Areas in gray are not visible from the satellite orbit. Areas in any other color are visible/covered from the satellite orbit. 


\section{4: Washington, D.C. and Montevideo: 90-Day, 3-Satellites, Altitude-Constrained}

\subsection{1: Description}

For this example, three satellites in orbit are maneuvered to improve the total coverage time over Washington, D.C., USA and Montevideo, Uruguay over the course of 90 days while subject to a maximum altitude constraint of $0.3 \mathrm{ER}$ above the Earth's mean surface. This example is designed to account for a realworld constraint which would often require a satellite to remain within a certain distance of the surface of the Earth. This could arise for any number of reasons such as focal length limits for optical equipment, electrical power constraints, exposure to Van Allen radiation, etc.

To address this scenario, the optimization problem seeks to find the nondominated surface that: (1) maximizes the coverage time over Washington, D.C.; (2) maximizes the coverage time over Montevideo; and (3) minimizes the propellant consumed by the entire constellation. This is the same optimization problem as in Example 6.3: Washington, D.C. and Montevideo: 90-Day, 3-Satellites except with the addition of the maximum altitude constraint of $0.3 \mathrm{ER}$ above the Earth's mean surface, which is equivalent to a maximum radial constraint of $1.3 \mathrm{ER}$.

A graphical depiction of the scenario at the initial date is shown in Fig. 62 . The evolutionary algorithm settings used for this example are listed in Table 11. The properties for the scenario are summarized in Table 12.

\subsection{2: Results and Analysis}

The nondominated surface from the final generation of the optimization is shown in Fig. 59. The original unoptimized solution is visible in the figure at the point of zero propellant consumption. That solution provides a total coverage time of 108.0 hours for Washington, D.C. and 36.0 hours for Montevideo. Three clusters of solutions have been marked on the nondominated surfaces and will be discussed below. The final nondominated surface is listed in Table 20 of Appendix C.

The original unoptimized solution is Solution 141 from Table 20. It is the same unoptimized solution from Example 6.3 which was previously discussed.

The result which maximizes total coverage time over Washington, D.C. is Solution 1 from Table 20. This solution raises the total coverage time of Washington, D.C. from 108.0 hours to 169.9 , and of Montevideo from 36.0 hours to 90.4 hours. This solution has all three constellation satellites executing the maneuver described in Section 4.10: Maximization of the Rate-of-Change of the Semi-Major Axis and Eccentricity while keeping the Argument of Periapsis Constant. For Satellites 1 and 2, the orbits are made eccentric with initial angles of periapsis separated by approximately 160 degrees. The solution has the three satellites consuming $20.5 \mathrm{~kg}$ out of the total available propellant of $30 \mathrm{~kg}$. 
Specifically, Satellites 1 and 2 consume all $10 \mathrm{~kg}$ of their available propellant while Satellite 3 only consumes $0.5 \mathrm{~kg}$ of its propellant. By the time Satellite 3 finishes executing the maneuver after consuming $0.5 \mathrm{~kg}$, its apoapsis, which began at $1.29 \mathrm{ER}$, has nearly reached the maximum altitude constraint of 1.3 ER. The 90-day evolution of the visibility map is shown in Fig. 132. The initial visibility map was described above. By Day 30 , all of the maneuvering has been completed, and the constellation's visibility footprints cover nearly all of Washington, D.C.'s latitude. The change in Satellite 3's visibility footprint is not readily apparent, which is consistent with the little propellant that it consumed.

The result which maximizes total coverage time over Montevideo is Solution 96 from Table 20. This solution raises the total coverage time of Washington, D.C. from 108.0 hours to 130.4 hours, and of Montevideo from 36.0 hours to 115.9 hours. This solution has Satellite 1 executing the maneuver described in Section 4.4: Maximization of the Rate-of-Change of the Semi-Major Axis, Satellite 2 executing the maneuver described in Section 4.10: Maximization of the Rate-of-Change of the Semi-Major Axis and Eccentricity while keeping the Argument of Periapsis Constant, and Satellite 3 executing the minimizing version of the maneuver described in Section 4.5: Maximization of the Rate-of-Change of Eccentricity. The solution has the three satellites consuming $29.9 \mathrm{~kg}$ out of the total available propellant of $30 \mathrm{~kg}$. The 90 -day evolution of the visibility map is shown in Fig. 133. The initial visibility map was described above. By Day 30, all of the maneuvering has been completed, and the constellation's visibility footprints cover most of Montevideo's latitude. Satellite 3's maneuver causes the satellite's two pre-existing swaths of coverage to increase in longitudinal width. Over the remaining 60 days, the high-visibility regions underneath the apoapsides of Satellites 1 and 2 oscillate across Montevideo's latitude due to the second-order gravity effects.

Of particular interest are the three clusters of solutions: Clusters $1 \mathrm{~b}, 3$, and 4 . One end of Cluster $1 \mathrm{~b}$ is anchored at the original unoptimized solution, while the other end maximizes coverage over Washington, D.C. This cluster is a truncated version of Cluster 1 from Example 6.3. Cluster 1 provides more coverage of Washington, D.C. due to the lack of the maximum altitude constraint from this example. The spans of Clusters 3 and 4 are most evident when inspecting the plot of propellant consumption versus Montevideo coverage in Fig. 59d. Cluster 3 extends the coverage of Montevideo beyond that provided by Cluster $1 \mathrm{~b}$. Similarly, Cluster 4 extends the coverage beyond that of Cluster 3 and does so all the way up to the maximum propellant consumption of $30 \mathrm{~kg}$. As visible in the coverage plot for both cities in Fig. 59b, Montevideo's improved coverage comes at the expense of Washington, D.C.'s coverage. Figs. 59b and $59 \mathrm{c}$ both indicate that distinct jumps in coverage and propellant are occurring between the clusters. Inspection of the maneuvers within each cluster partially explains the source of these jumps.

Most of the solutions in Cluster $1 \mathrm{~b}$ are characterized by the following: 
- Satellite 3 performs little to no maneuvering (i.e. less than $1 \mathrm{~kg}$ of propellant consumption)

- Satellite 1 performs little to no maneuvering (i.e. less than $1 \mathrm{~kg}$ of propellant consumption) when the constellation's propellant consumption is between 0 and $10 \mathrm{~kg}$.

Most of the solutions in Clusters 3 and 4 are characterized by the following:

- Satellites 1 and 2 execute either Maximization of the Rate-of-Change of the Semi-Major Axis, Maximization of the Rate-of-Change of the SemiMajor Axis and Eccentricity while keeping the Argument of Periapsis Constant, or Maximization of the Rate-of-Change of the Semi-Major Axis while keeping the Eccentricity constant while using nearly all of the available propellant.

- Satellite 3 executes the minimizing version of the Maximization of the Rate-of-Change of Eccentricity maneuver.

What mostly distinguishes Cluster 3 from 4 is the amount of propellant consumed by Satellite 3 while executing its maneuver. Recall, that in Cluster $1 \mathrm{~b}$, Satellite 3 consumes little to no propellant. In Cluster 3 , the propellant consumption jumps to between 3.9 and $5.3 \mathrm{~kg}$. In Cluster 4, the propellant consumption jumps to $8.5 \mathrm{~kg}$ and higher.

Inspection of Cluster 1b within Fig. 59 shows that improvement in coverage of one city also improves coverage of the other. Within this cluster, Satellite 3 performs little to no maneuvering. However, Clusters 3 and 4 show that improved coverage of Montevideo is obtainable at the expense of coverage over Washington, D.C. Inspection of the maneuvers in Clusters 3 and 4 reveals that Satellite 3 minimizes its eccentricity which causes its orbit to become more circular. It is interesting to compare these results with those obtained in Example 6.3's Cluster 1. In order to obtain the same amount of coverage over Montevideo, Satellite 3 executed the maneuver described in Section 4.7: Maximization of the Rate-of-Change of the Semi-Major Axis while keeping the Eccentricity constant. This maneuver forces the entire orbit, including the apoapsis, to increase in size. However, the addition of the radial constraint in this example, at 1.3 ER, inhibits Satellite 3, whose initial apoapsis is 1.29 ER, from fully executing that maneuver.

Satellite 3 must be maneuvered in a way that does not violate the maximum altitude constraint. The maneuver which achieves this while still providing a nondominated solution is the minimizing version of the Maximization of the Rateof-Change of Eccentricity maneuver. This maneuver causes the orbit to become more circular and thus spend less time over the Northern Hemisphere. As a result, coverage over Montevideo increases (see Fig. 59d) while coverage over Washington, D.C. decreases (see Fig. 59c). 


\subsection{3: Summary}

The results from the optimization indicate that three clusters of solutions are found to exist. The maneuvers in Cluster 1 improve coverage over both Washington, D.C. and Montevideo by inhibiting Satellite 3's maneuvering because Satellite 3's initial apoapsis of 1.29 ER is near the maximum radial constraint of 1.3 ER. It is within this cluster that the maximum coverage over Washington, D.C. is found. Note that this cluster is a truncated version of Cluster 1 from Example 6.3 which provides more coverage of Washington, D.C. due to the lack of the maximum altitude constraint from this example.

Inspection of Fig. 59d reveals that the maneuvering solutions in Cluster 3 extends the coverage of Montevideo beyond that provided by Cluster $1 \mathrm{~b}$.

Similarly, Cluster 4 extends the coverage beyond that of Cluster 3 and does so all the way up to the maximum propellant consumption of $30 \mathrm{~kg}$. These results show that this coverage improvement comes at the expense of

Washington, D.C.'s coverage because the reduction in Satellite 3's eccentricity reduces the amount of time that Satellite 3 spends over the Northern

Hemisphere. 


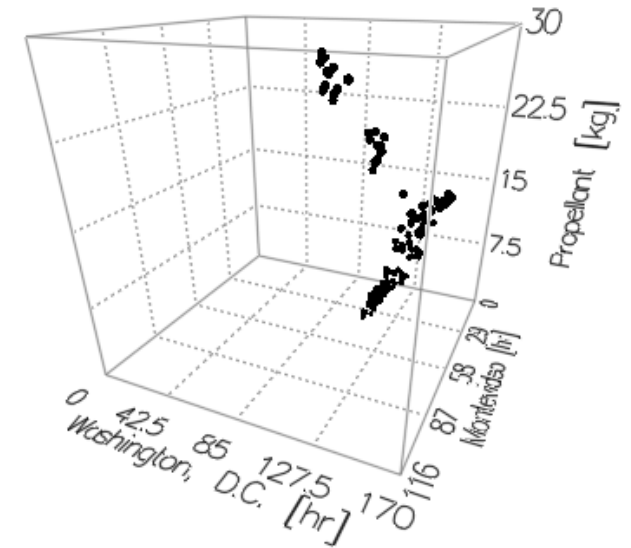

(a)

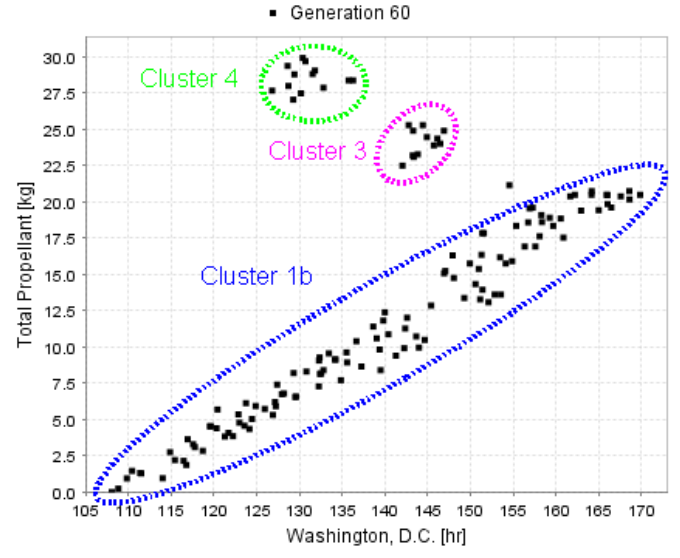

(c)

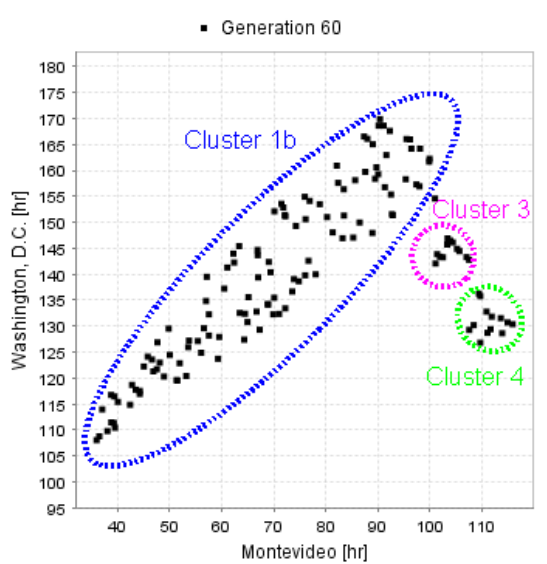

(b)

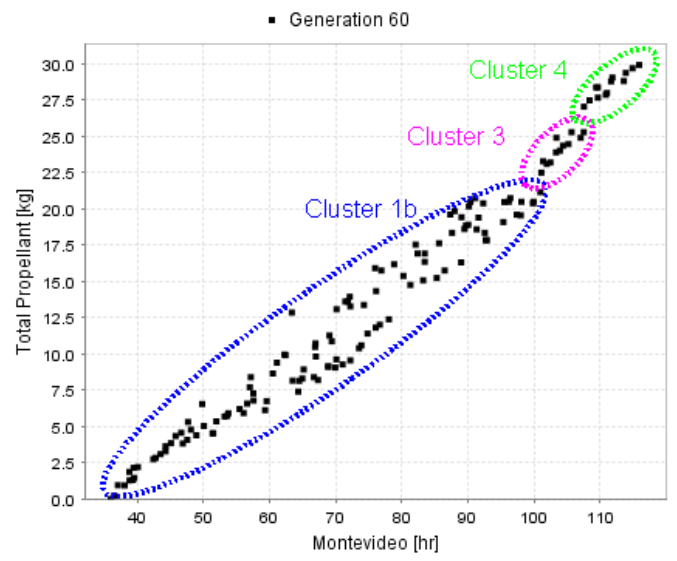

(d)

Figure 59: The final generation of nondominated solutions for Example 6.4 are shown within objective space. Figure (a) shows the solutions in 3D space. Figures (b), (c), and (d) show the 3D solutions projected onto three 2D planes. As visible in the figures, the total coverage time provided by the unoptimized constellation is 108.0 hours for Washington, D.C. and $\mathbf{3 6 . 0}$ hours for Montevideo. Three sets of solutions (Clusters $1 \mathrm{~b}, 3$, and 4) have been circled each of which reach different areas of the objective space. 

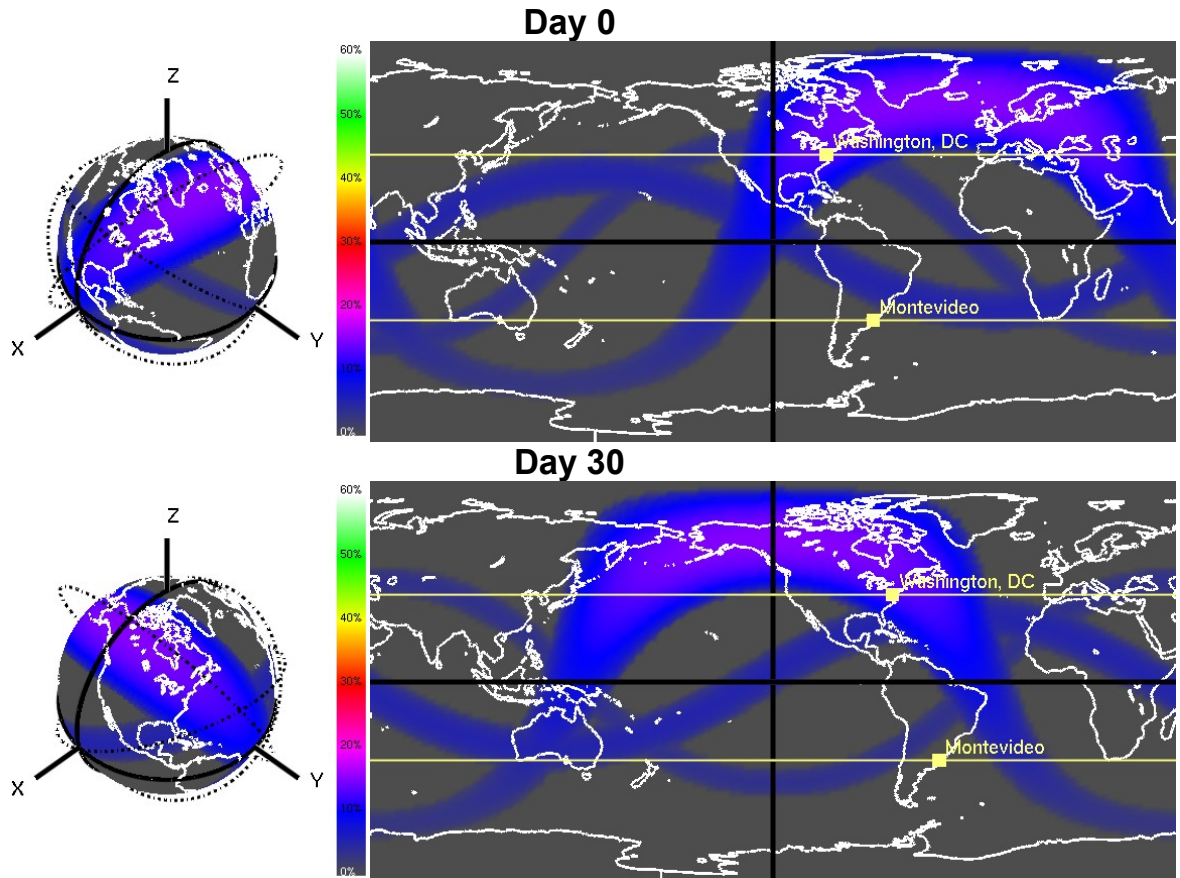

Day 60
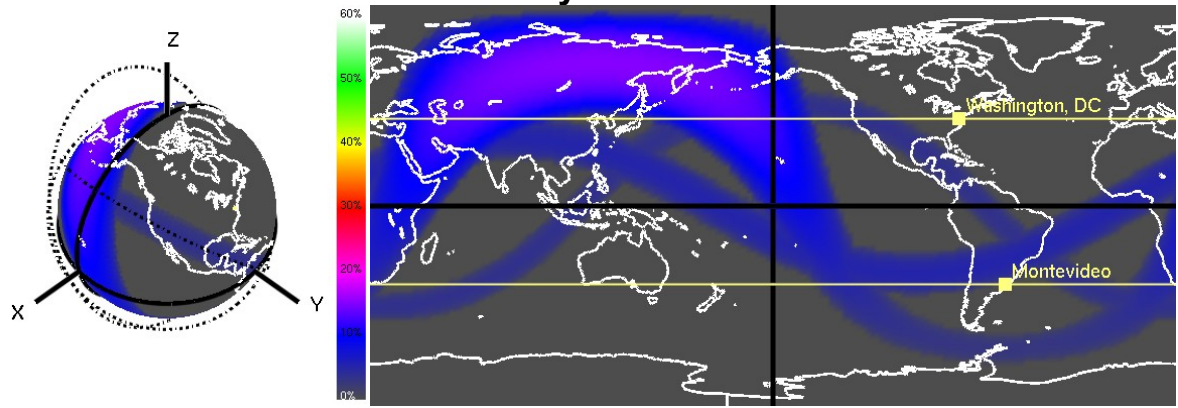

\section{Day 90}
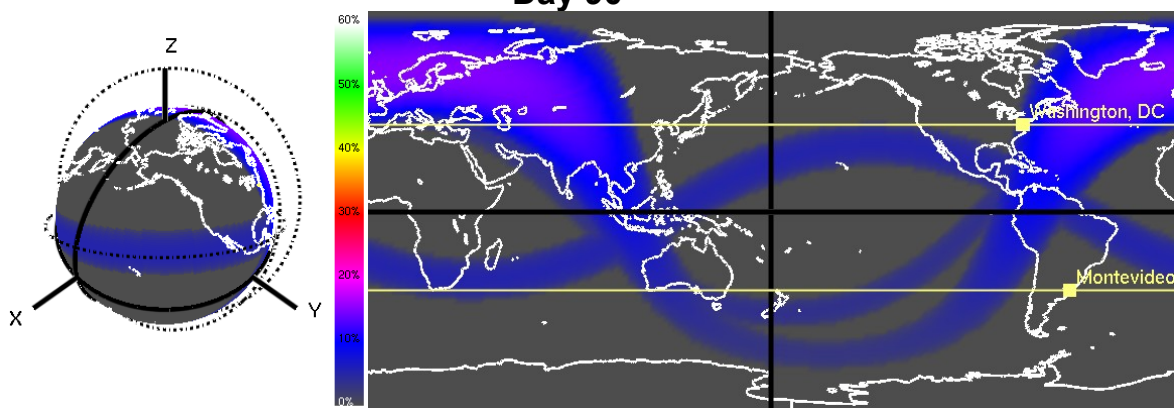

Figure 60: Shown is the 90-day evolution of the visibility map for the original unoptimized solution (Solution 141) of Example 6.4. This solution has the satellite executing no maneuvers, and results in a total coverage of 108.0 hours for Washington, D.C. and 36.0 hours for Montevideo. Washington, D.C., Montevideo, and their corresponding latitudes are marked in yellow. The continental outlines are marked in white. Areas in gray are not visible from the satellite orbit. Areas in any other color are visible/covered from the satellite orbit. 

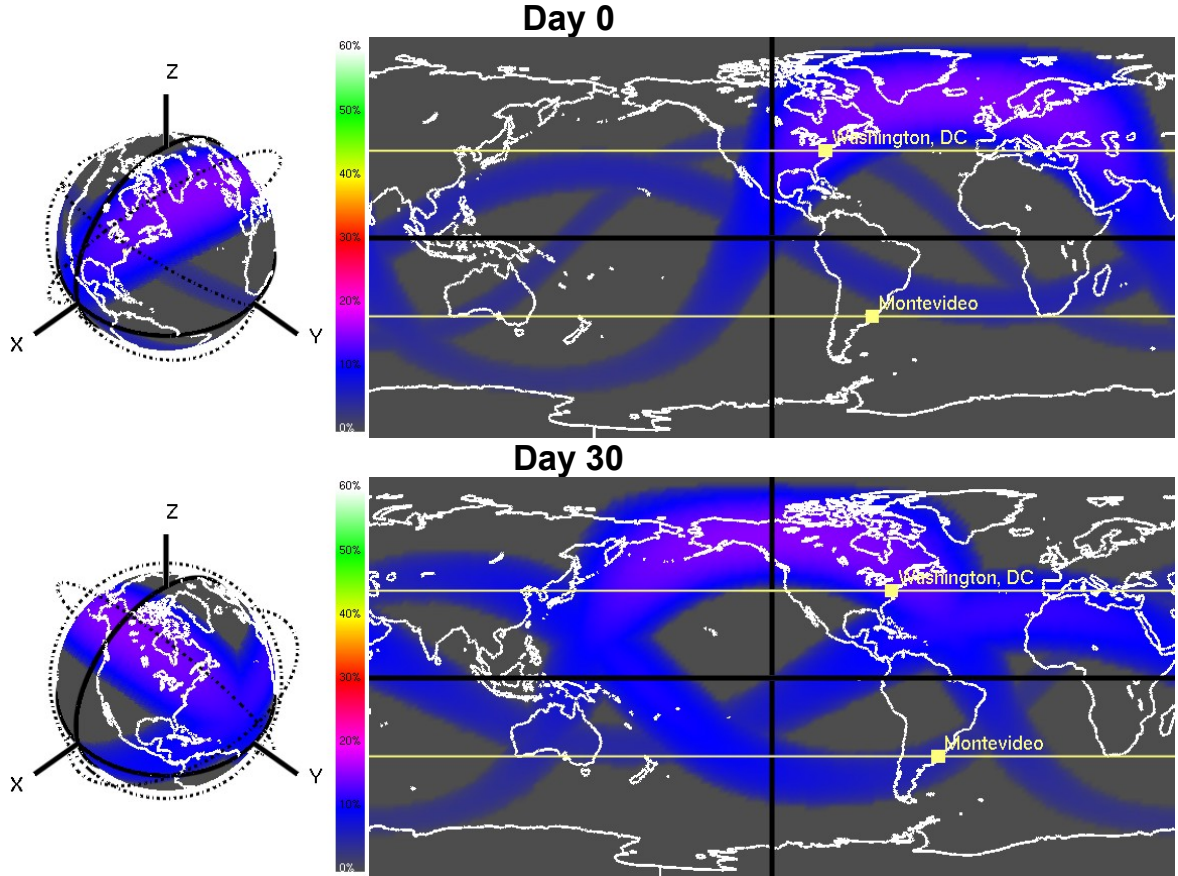

Day 60
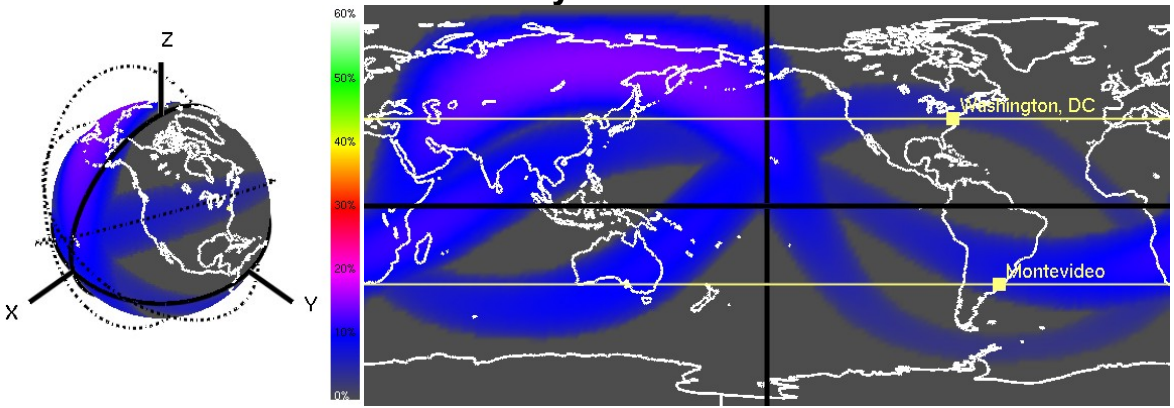

\section{Day 90}
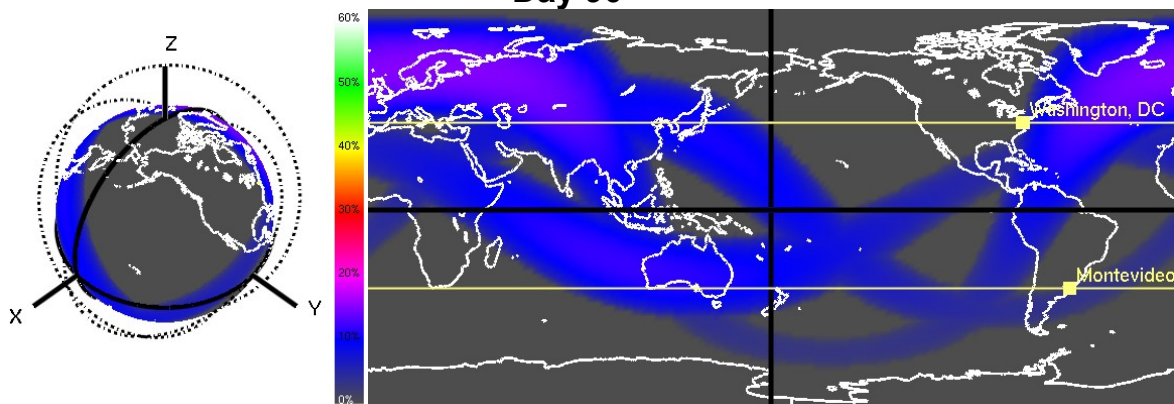

Figure 61: Shown is the 90-day evolution of the visibility map for Solution 1 of Example 6.4. This solution has the satellite executing a maneuver that maximizes coverage over Washington, D.C. of 169.9 hours; Montevideo's coverage is 90.4 hours. (For comparison, the total coverage time provided by the unoptimized satellite is 108.0 hours for Washington, D.C. and 36.0 hours for Montevideo.) Washington, D.C., Montevideo, and their corresponding latitudes are marked in yellow. The continental outlines are marked in white. Areas in gray are not visible from the satellite orbit. Areas in any other color are visible/covered from the satellite orbit. 

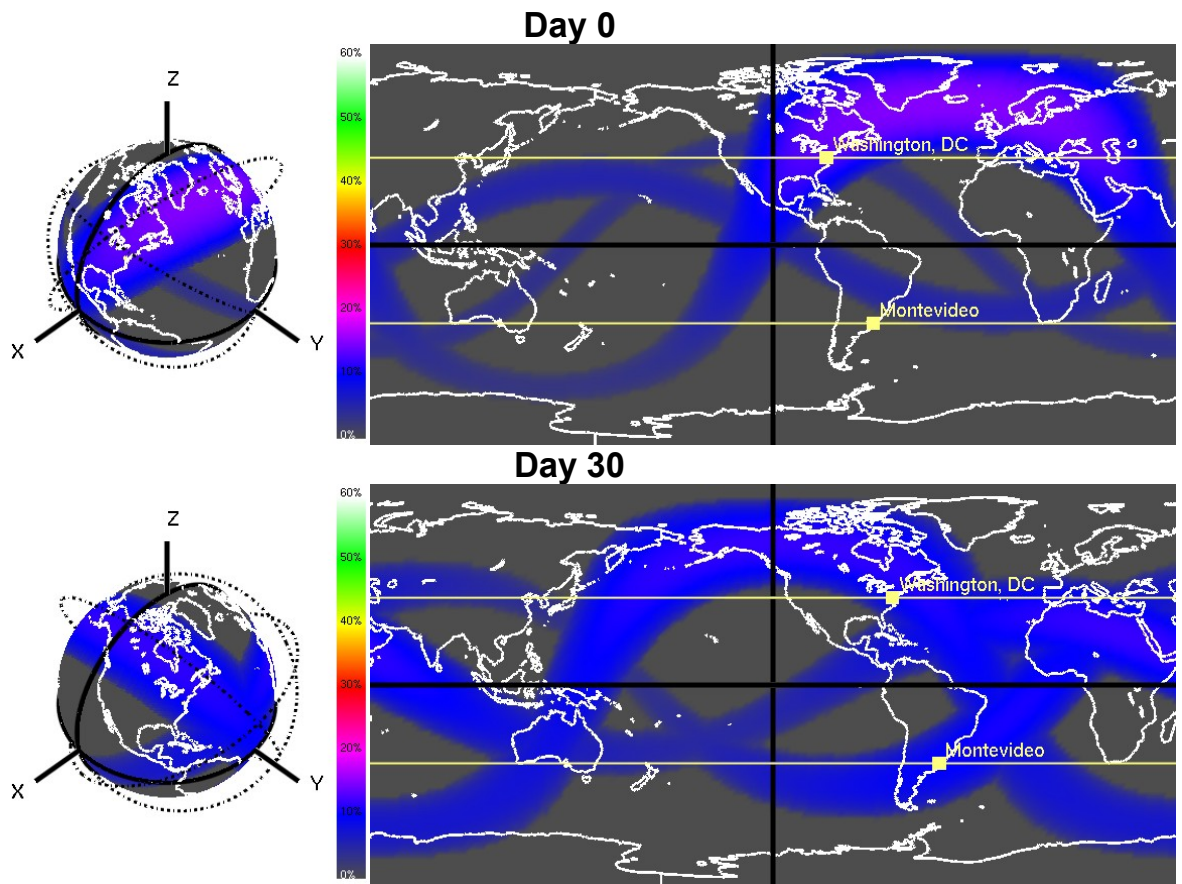

Day 60
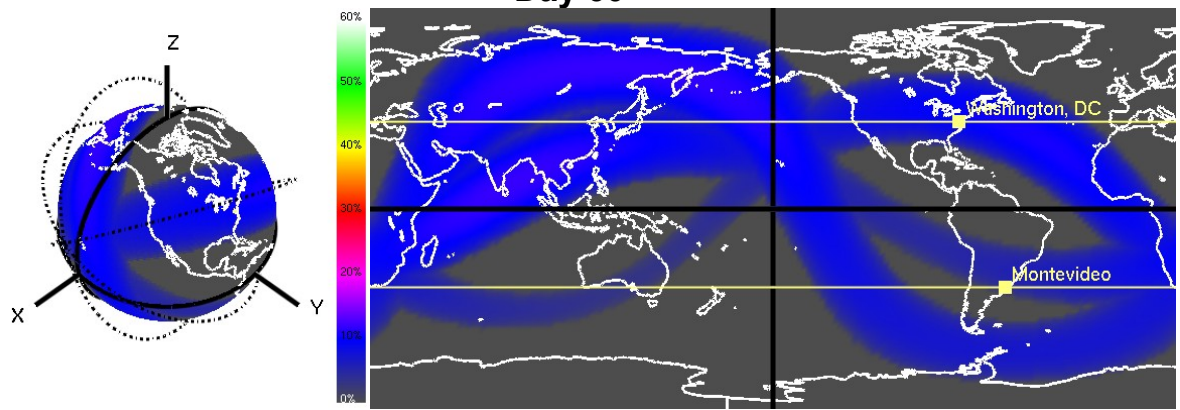

\section{Day 90}
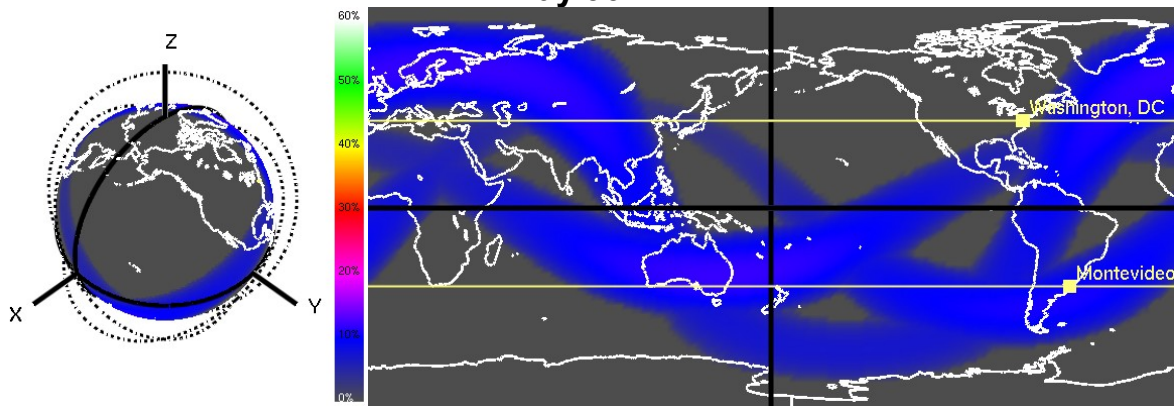

Figure 62: Shown is the 90-day evolution of the visibility map for Solution 96 of Example 6.4. This solution has the satellite executing a maneuver that maximizes coverage over Montevideo of 115.9 hours; Washington D.C.'s coverage is 130.4 hours. (For comparison, the total coverage time provided by the unoptimized satellite is 108.0 hours for Washington, D.C. and $\mathbf{3 6 . 0}$ hours for Montevideo.) Washington, D.C., Montevideo, and their corresponding latitudes are marked in yellow. The continental outlines are marked in white. Areas in gray are not visible from the satellite orbit. Areas in any other color are visible/covered from the satellite orbit. 


\section{5: Summary of Examples}

Example 6.1 addressed reconfiguring a single satellite for improved coverage of two targets at similar latitudes (Washington, D.C. and Beijing). The results along the nondominated surface indicated that the maneuvering strategies which improved coverage of one target also improved coverage of the other target. The results also showed that obtaining more coverage required consuming more propellant during maneuvering.

Example 6.2 addressed reconfiguring a single satellite for improved coverage of Washington, D.C. and Montevideo. The results from the optimization indicate that a range of nondominated solutions are found to exist that balance the coverage between both targets. Within parameter space, the maneuvers that provide the best coverage over either target were those that drove the satellite orbit into becoming elliptical. Since the resulting elliptical orbits were greatly affected by rotation of the apsides, the orbits were initially maneuvered in such a way as to take advantage of these effects.

Example 6.3 addressed reconfiguring three satellites for improved coverage of the same two targets as in Example 6.2. The results from the optimization indicate that two clusters of nondominated solutions are found to exist that favor either Washington D.C. (Cluster 2) or Montevideo (Cluster 1). Each of the clusters contains a set of maneuvering strategies that are somewhat distinct from the other. However, they both share the correlation that the greatest coverage over either target is only attainable by consuming all of the available propellant.

Example 6.4 addressed the same scenario as in Example 6.3 except with an additional constraint on the maximum altitude that any satellite may attain. The results from the optimization indicate that three clusters of nondominated solutions are found to exist. Cluster $1 \mathrm{~b}$ contains solutions that improve coverage of both cities. This cluster is also a truncated version of Cluster 1 from Example 6.3. The truncation occurs because the Cluster 1 solutions which are absent from Cluster $1 \mathrm{~b}$ have Satellite 3 maneuvering in a way that violates the maximum altitude constraint. In contrast, the solutions in Clusters 3 and 4 have Satellite 3 maneuvering in a way that does not violate the constraint. Those maneuvers, however, increase coverage of Montevideo while decreasing coverage of Washington, D.C. Another interesting observation regarding the coverage-maximizing solution for Washington, D.C. (located at one end of Cluster $1 \mathrm{~b}$ ) is that it does not require consuming all of the constellation's available propellant. This is in marked contrast to the nondominated solutions obtained in the other examples where the maximizing coverage for either city required consuming nearly all of the available propellant.

All of the examples use visibility maps at different instants to visually characterize the coverage provided by the satellite constellation. Inspecting any individual map quickly identifies which latitudes have better coverage than others. Inspecting the visibility maps over time also reveals how the secondorder gravity effects alter the satellites' orbits and the coverage that they provide. 


\section{Chapter 7: Summary}

This work has focused on reconfiguring existing satellite constellations in order to improve coverage of multiple targets on the surface of the Earth. Chapter 1: Introduction described how the focus of this work differs from other work on satellite constellations. It also described the constellation reconfiguration as a multiobjective optimization problem.

Chapter 2: Calculating Coverage described the models used to describe the physical elements essential to calculating coverage. This included the model for the satellite motion, description of the Earth's surface, planetary rotation, line-ofsight between a target and a satellite, visibility schedules, and figures-of-merit.

Chapter 3: Estimating Satellite Coverage described the relationship between orbital geometry and the coverage that a satellite provides. This was done by inspecting the line-of-sight cones emanating from locations on Earth-centered virtual spheres and identifying what portions of a satellite orbit were visible. These orbital views were then displayed as color-coded visibility maps on a virtual inertial sphere. The rotation of the Earth through/underneath these visibility maps illustrated patterns of coverage provided by a satellite over the course of a sidereal day. Finally, estimates of coverage provided by a single satellite were shown to be obtainable by aggregating this geometrical information from across a target's inertial latitude.

Chapter 4: Maneuvering Strategies for Maximal Changes in Orbital Geometry used Gauss' variational equations to arrive at a set of maneuvering strategies that effect maximal changes to different properties of a satellite orbit's geometry.

Chapter 5: Optimizing Constellation Coverage by Performing Maximal Changes to Orbital Geometry revisited the original optimization problem framed in Chapter 1. A new multiobjective optimization problem was formed using the results from Chapters 3 and 4 . In it, discrete maneuvering strategies and continuous propellant allotments for each satellite became the parameters to be varied within the optimization problem. A novel multiobjective evolutionary algorithm is described that is used to address this problem.

Chapter 6: Examples described a set of examples that are presented to demonstrate the utility of satellite reconfiguration on improving coverage over multiple targets on the surface of the Earth. The examples were optimized using the algorithm described in Chapter 5, and several nondominated surfaces were obtained. These nondominated surfaces showed how coverage over all targets were improved with orbital maneuvering. 


\section{Chapter 8: Appendices}

\section{Appendix A: Summary of Operational Satellites}

This Appendix surveys the satellites currently in orbit. The Union of Concerned Scientists (UCS) has compiled data pertaining to all operational satellites in orbit as of September $10,2006^{58}$. This section categorizes the data in various ways ${ }^{\mathrm{N}}$.

\section{Nomenclature}

LEO Low-Earth Orbit; apoapsis altitude $\leq 2000 \mathrm{~km}$ (i.e. whose periods are 2.12 hours or less)

MEO12 Medium-Earth Orbit; apoapsis altitude $>2000 \mathrm{~km}$ and whose periods are 12 hours or less

GEO Geosynchronous Orbit; circular orbits with 24-hour periods

OTHER All others

$\mathrm{N}$ Two corrections to the data have been made:

(a) Superbird-A2 (Superbird-6)'s inclination is listed as 66 degrees by the UCS data whereas its inclination is listed as 1.89 degrees according to http://www.kosmo.cz/modules.php?op=modload\&name=kosmo\&file=index\&fil=/s/2004/011A.HTM

(b) TDRS-8's inclination is listed as 4 degrees by the UCS data whereas its inclination is listed as 1.4 degrees according to http://heavensabove.com/satinfo. $a s p ?$ lat $=0$ \&lng $=0 \&$ alt $=0 \& l o c=U n s p e c i f i e d \& T Z=C E T \& S a t \mid D=26388$ 


\section{Types of Satellite Orbits}

The following table categorizes all of the satellites by orbit type: LEO, MEO12, GEO, or OTHER. In parenthesis is shown the number of satellites in that category as a fraction of the total number of satellites.

\begin{tabular}{|c|c|c|c|c|c|}
\hline Type of Satellite & IFO & ME012 & GEO & OTHFR & Tatal \\
\hline Number of Satellites & $\begin{array}{c}385 \\
(47 \%)\end{array}$ & $\begin{array}{c}76 \\
(9 \%)\end{array}$ & $\begin{array}{c}349 \\
(42 \%)\end{array}$ & $\begin{array}{c}17 \\
(2 \%)\end{array}$ & $\begin{array}{c}827 \\
(100 \%)\end{array}$ \\
\hline
\end{tabular}

\section{Eccentricity by Type of Satellite Orbit}

The following table categorizes those satellites in eccentric orbits by orbit type: LEO, MEO12, GEO, or OTHER. In parenthesis is shown the number of satellites in eccentric orbits as a fraction of the total number of satellites for that orbit type.

Table 14: Eccentricity by Type of Satellite Orbit

\begin{tabular}{|c|c|c|c|c|}
\hline Type of Orbit & LEO & MEO12 & GEO & OTHER \\
\hline $\begin{array}{c}\text { Number of satellites } \\
\text { whose orbit has an }\end{array}$ & 3 & 29 & 0 & 16 \\
eccentricity $>0.05$ & $(1 \%)$ & $(38 \%)$ & $(0 \%)$ & $(94 \%)$ \\
\hline
\end{tabular}




\section{Inclination by Type of Satellite Orbit}

The following table categorizes the number of the satellites by the satellite's orbital inclination in degrees and by the satellite's type of orbit. The rows categorize the satellites into orbit types: LEO, MEO12, GEO, and OTHER. The number of satellites within each orbit type categorization is shown. The satellites are further categorized by whether the orbit is circular (eccentricity $\leq 0.05$ ) or elliptical (eccentricity $>0.05$ ). The columns categorize the satellites by the satellite's orbital inclination in degrees. The "(" symbol indicates the adjacent value is exclusive, and the "[" and "]" symbols indicate that the adjacent value is inclusive. In parenthesis is shown the number of satellites as a fraction of the total number of satellites for that row.

Table 15: Inclination by Type of Satellite Orbit

\begin{tabular}{|c|c|c|c|c|c|c|c|c|c|c|c|}
\hline $\begin{array}{l}\text { Orbit } \\
\text { Type } \\
\text { Incl. } \\
\text { [deg] }\end{array}$ & {$[0,1]$} & $(1,10]$ & $(10,20]$ & $(20,40]$ & $(40,60]$ & $(60,80]$ & $(80,100]$ & $(100,120]$ & $(120,140]$ & $(140,160]$ & $(160,180]$ \\
\hline $\begin{array}{l}\text { LEO } \\
\text { All }\end{array}$ & 0 & $\begin{array}{l}1 \\
(0 \%)\end{array}$ & 0 & \begin{tabular}{|l|}
10 \\
$(3 \%)$
\end{tabular} & $\begin{array}{l}80 \\
(21 \%)\end{array}$ & $\begin{array}{l}41 \\
(11 \%)\end{array}$ & $\begin{array}{l}248 \\
(64 \%)\end{array}$ & $\begin{array}{l}4 \\
(1 \%)\end{array}$ & 0 & $\begin{array}{l}1 \\
(0 \%)\end{array}$ & 0 \\
\hline $\begin{array}{l}\text { LEO } \\
\text { Circular }\end{array}$ & 0 & 1 & 0 & 10 & 80 & 41 & 245 & 4 & 0 & 1 & 0 \\
\hline $\begin{array}{l}\text { LEO } \\
\text { Eccentric }\end{array}$ & 0 & 0 & 0 & 0 & 0 & 0 & 3 & 0 & 0 & 0 & 0 \\
\hline $\begin{array}{l}\text { MEO12 } \\
\text { All }\end{array}$ & 0 & 0 & 0 & $\begin{array}{l}1 \\
(1 \%)\end{array}$ & $\begin{array}{l}30 \\
(39 \%)\end{array}$ & $\begin{array}{l}40 \\
(53 \%)\end{array}$ & $\begin{array}{l}2 \\
(3 \%)\end{array}$ & $\begin{array}{l}3 \\
(4 \%)\end{array}$ & 0 & 0 & 0 \\
\hline $\begin{array}{l}\text { MEO12 } \\
\text { Circular }\end{array}$ & 0 & 0 & 0 & 0 & 30 & 17 & 0 & 0 & 0 & 0 & 0 \\
\hline $\begin{array}{l}\text { MEO12 } \\
\text { Eccentric }\end{array}$ & 0 & 0 & 0 & 1 & 0 & 23 & 2 & 3 & 0 & 0 & 0 \\
\hline $\begin{array}{l}\text { GEO } \\
\text { All }\end{array}$ & $\begin{array}{l}289 \\
(83 \%)\end{array}$ & $\begin{array}{l}54^{\circ} \\
(15 \%)\end{array}$ & $\begin{array}{l}6 \\
(2 \%)\end{array}$ & 0 & 0 & 0 & 0 & 0 & 0 & 0 & 0 \\
\hline
\end{tabular}

O Includes two inclinations that were corrected from the UCS database 


\begin{tabular}{|c|c|c|c|c|c|c|c|c|c|c|c|}
\hline $\begin{array}{l}\text { Orbit } \\
\text { Type } \\
\text { Incl. } \\
\text { [deg] }\end{array}$ & {$[0,1]$} & $(1,10]$ & $(10,20]$ & $(20,40]$ & $(40,60]$ & $(60,80]$ & $(80,100]$ & $(100,120]$ & $(120,140]$ & $(140,160]$ & $(160,180]$ \\
\hline $\begin{array}{l}\text { GEO } \\
\text { Circular }\end{array}$ & 289 & 54 & 6 & 0 & 0 & 0 & 0 & 0 & 0 & 0 & 0 \\
\hline $\begin{array}{l}\text { GEO } \\
\text { Eccentric }\end{array}$ & 0 & 0 & 0 & 0 & 0 & 0 & 0 & 0 & 0 & 0 & 0 \\
\hline $\begin{array}{l}\text { OTHER } \\
\text { All }\end{array}$ & 0 & 0 & 0 & \begin{tabular}{|l}
6 \\
$(35 \%)$
\end{tabular} & $\begin{array}{l}2 \\
(12 \%)\end{array}$ & $\begin{array}{l}3 \\
(18 \%)\end{array}$ & \begin{tabular}{|l}
6 \\
$(35 \%)$
\end{tabular} & 0 & 0 & 0 & 0 \\
\hline $\begin{array}{l}\text { OTHER } \\
\text { Circular }\end{array}$ & 0 & 0 & 0 & 0 & 1 & 0 & 0 & 0 & 0 & 0 & 0 \\
\hline $\begin{array}{l}\text { OTHER } \\
\text { Eccentric }\end{array}$ & 0 & 0 & 0 & 6 & 1 & 3 & 6 & 0 & 0 & 0 & 0 \\
\hline
\end{tabular}




\section{Propellant Mass as a Percentage of Launch Mass}

The following table categorizes the number of the satellites by their type of orbit, and by the initial propellant mass as a fraction of the initial total mass. The rows categorize the satellites into orbit types: LEO, MEO12, GEO, and OTHER. The columns categorize the satellites by the initial propellant mass as a fraction of the initial total mass; the "(" symbol indicates the adjacent value is exclusive, and the "l" symbol indicates that the adjacent value is inclusive. In parenthesis is shown the number of satellites as a fraction of the total number of satellites for that row.

Table 16: Propellant Mass as a Percentage of Launch Mass ${ }^{p}$

\begin{tabular}{|c|c|c|c|c|c|c|c|c|c|c|c|}
\hline $\begin{array}{l}\text { Orbit Type } \\
\text { IMass \% }\end{array}$ & [0] & $(0,10]$ & $(10,20]$ & $(20,30]$ & $(30,40]$ & $(40,50]$ & $(50,60]$ & $(60,70]$ & $(70,80]$ & $(80,90]$ & $(90,100]$ \\
\hline $\mathrm{LEO}^{\mathrm{Q}}$ & $\begin{array}{l}5 \\
(2 \%) \\
\end{array}$ & $\begin{array}{l}13 \\
(6 \%) \\
\end{array}$ & $\begin{array}{l}90^{R} \\
(42 \%) \\
\end{array}$ & $\begin{array}{l}7 \\
(3 \%)\end{array}$ & $\begin{array}{l}9 \\
(4 \%)\end{array}$ & $\begin{array}{l}5 \\
(2 \%) \\
\end{array}$ & $\begin{array}{l}83^{5} \\
(39 \%)\end{array}$ & $\begin{array}{l}1 \\
(0 \%)\end{array}$ & 0 & $\begin{array}{l}2 \\
(1 \%)\end{array}$ & 0 \\
\hline MEO12 ${ }^{\top}$ & 0 & 0 & $\begin{array}{l}14 \\
(3 \%)\end{array}$ & 0 & 0 & $\begin{array}{l}3 \\
(9 \%) \\
\end{array}$ & $\begin{array}{l}29 \\
(88 \%) \\
\end{array}$ & 0 & 0 & 0 & 0 \\
\hline GEOV & 0 & $\begin{array}{l}2^{W} \\
(1 \%)\end{array}$ & $\begin{array}{l}5^{\mathrm{X}} \\
(2 \%)\end{array}$ & $\begin{array}{l}11 \\
(4 \%)\end{array}$ & $\begin{array}{l}42^{Y} \\
(15 \%)\end{array}$ & $\begin{array}{l}87^{Z} \\
(32 \%)\end{array}$ & $\begin{array}{l}117^{\mathrm{AA}} \\
(43 \%)\end{array}$ & $\begin{array}{l}7 \\
(3 \%)\end{array}$ & 0 & 0 & 0 \\
\hline
\end{tabular}

$\mathrm{P}$ Propellant mass is assumed to be the difference between the satellite's launch mass and dry mass

Q Data available for 215 (56\%) of the satellites of this type

$\mathrm{R}$ One approximate measurement used

$S$ One approximate measurement used

T Data available for 33 (42\%) of the satellites of this type

$\mathrm{U}$ One approximate measurement used

V Data available for $271(78 \%)$ of the satellites of this type

W One beginning-of-life measurement used

$X$ One beginning-of-life measurement used

$Y$ Four beginning-of-life measurements used

$Z$ Ten beginning-of-life measurements used

AAOne beginning-of-life measurement used 


\begin{tabular}{|l|l|l|l|l|l|l|l|l|l|l|l|}
\hline $\begin{array}{c}\text { Orbit Type } \\
\text { IMass \% }\end{array}$ & \multicolumn{1}{|c|}{$[\mathbf{0}]$} & $\mathbf{( 0 , 1 0 ]}$ & $\mathbf{( 1 0 , 2 0 ]}$ & $\mathbf{( 2 0 , 3 0 ]}$ & $\mathbf{( 3 0 , 4 0 ]}$ & $\mathbf{( 4 0 , 5 0 ]}$ & $\mathbf{( 5 0 , 6 0 ]}$ & $\mathbf{( 6 0 , 7 0 ]}$ & $\mathbf{( 7 0 , 8 0 ]}$ & $\mathbf{( 8 0 , 9 0 ]}$ & $\mathbf{( 9 0 , 1 0 0 ]}$ \\
\hline OTHER $^{\mathrm{BB}}$ & 0 & $\begin{array}{l}4^{\mathrm{CC}} \\
(29 \%)\end{array}$ & $\begin{array}{l}2 \\
(14 \%)\end{array}$ & $\begin{array}{l}1 \\
(7 \%)\end{array}$ & 0 & 0 & $\begin{array}{l}7 \\
(50 \%)\end{array}$ & 0 & 0 & 0 & 0 \\
\hline
\end{tabular}

BBData available for $14(82 \%)$ of the satellites of this type

CCOne beginning-of-life measurements used 



\section{Appendix B: Software Modules}

This Appendix briefly describes the different software modules used to produce the results shown in this work.

\section{Ordinary Differential Equations (ODE)}

This module is capable of numerically integrating equations of motion by using the adaptive-step, Runge-Kutta-Fehlberg 4 th- $5^{\text {th }}$ order algorithm 32 .

\section{Optimization}

This module is capable of numerically solving different types of optimization problems. One of the problem types that it can solve is root-finding. Another problem type that it can solve is identifying the nondominated solutions among a set of solutions.

\section{Astrodynamics}

This module implements the equations of motion described in Section 2.1: Motion of Satellites in Orbit. This module uses the ODE module.

\section{Orbital Maneuvering}

This module implements the different maneuvers described in Chapter 4: Maneuvering Strategies for Maximal Changes in Orbital Geometry. This module uses the Astrodynamics and Optimization modules.

\section{Satellite Coverage}

This module implements the coverage calculations described in Section 2.3: Determining Visibility between Satellites and Targets. This module uses the Astrodynamics module.

\section{Parallel Computation of Coverage}

This module provides the infrastructure for computing coverage in parallel among multiple computers. This module uses the Satellite Coverage module.

This module follows the Master-Worker design pattern ${ }^{59}$. The Master runs on one computer and offers itself as a service willing to manage requests for the computation of satellite coverage. Workers run on other computers and accept job assignments from the Master. When the Workers complete the computation of coverage, the Master returns the result to the original requester.

\section{Multiobjective Evolutionary Algorithms (MOEA)}

This module contains the multiobjective evolutionary algorithms described in Chapter 5: Optimizing Constellation Coverage by Performing Maximal Changes to Orbital Geometry. This module uses the Optimization module. 
Multiobjective Evolutionary Algorithms (Customized for Coverage Computation)

This module integrates the multiobjective evolutionary algorithms with the parallel computation of coverage. This is motivated by two issues:

1. the fitness of any individual chromosome is independent of the other chromosomes

2. the fitness of each chromosome consists of two steps:

(a) calculating the visibility schedules subject to the maneuvering strategies specified in the chromosome

(b) calculating the multiple figures-of-merit (a.k.a. chromosome fitnesses) from the visibility schedules

Step 2(a) uses the Parallel Computation of Coverage module.

\section{Overview of Optimization Process}

Figure 63 shows the flow of information required to solve any one of the examples from Chapter 6: Examples. A scenario is initially defined by the satellite constellation, targets, and timespan of interest. This scenario is fed into the customized Multiobjective Optimization Algorithm module. First, this module creates the initial population of chromosomes. Each chromosome consists of the original scenario, along with a maneuvering strategy for each satellite. Each chromosome's fitness is determined in two steps, as described above. For Step 2(a), the visibility schedule is calculated by the Parallel Computation of Coverage module with the original scenario and the maneuvering strategies contained in that chromosome. Once calculated, the fitness/figure-of-merit can be calculated. After all of the chromosomes for a generation have been calculated, the multiobjective genetic algorithm performs the appropriate genetic operations (i.e. parent selection, crossover or cloning, and mutation) until a termination criterion has been satisfied.

\section{Third-Party Software}

\section{Java Development Kit}

The Java Development Kit (JDK) includes both the Java programming language as well as the Java Virtual Machine (JVM). The coding was done in the Java programming language and was compiled for running in the Java Virtual Machine. Sun Microsystems' implementation of the JDK is available at http://java.sun.com. The language's type- and error-checking has been superb at detecting errors throughout the development. Furthermore, Sun Microsystems' implementation of the JVM is both fast, free, cross-platform, and open-sourced as OpenJDK. 


\section{NetBeans Integrated Development Environment}

The NetBeans Integrated Development Environment (IDE) was used to develop nearly all of this work's code. It is open-sourced and available at http://netbeans.org. It has been a wonderful tool in easing and simplifying the development of the various modules.

JUnit

JUnit is a framework for unit-testing code. It is open-sourced and available at http://junit.org. It has proved to be an invaluable tool for simplifying the testing of various parts of this research.

\section{JFreeChart}

JFreeChart is a Java library for producing very good 2D plots. It is opensourced and available at http://jfree.org. It was used to produce most of the $2 \mathrm{D}$ plots in this work and was a great asset to have.

Java Bindings for OpenGL

Java Bindings for OpenGL (JOGL) is a library for portraying 3D objects in Java. It is open-sourced and available at http://jogl.dev.java.net. It was a wonderful asset which enabled the creation of the 2D and 3D visibility maps shown in this work.

\section{OpenOffice.org}

OpenOffice.org is an office suite containing such tools as a word processor, a spreadsheet, a database, and a presentation tool. It is open-sourced and available at http://openoffice.org. All of these components were used to write this work, its various incarnations as presentations, and most of its qualitative graphics.

\section{XStream}

$X$ Stream is a library for serializing Java object's into an XML stream. It is open-sourced and available at http://xstream.codehaus.org. It has proven to be very valuable at archiving objects while still being very easy to use. 


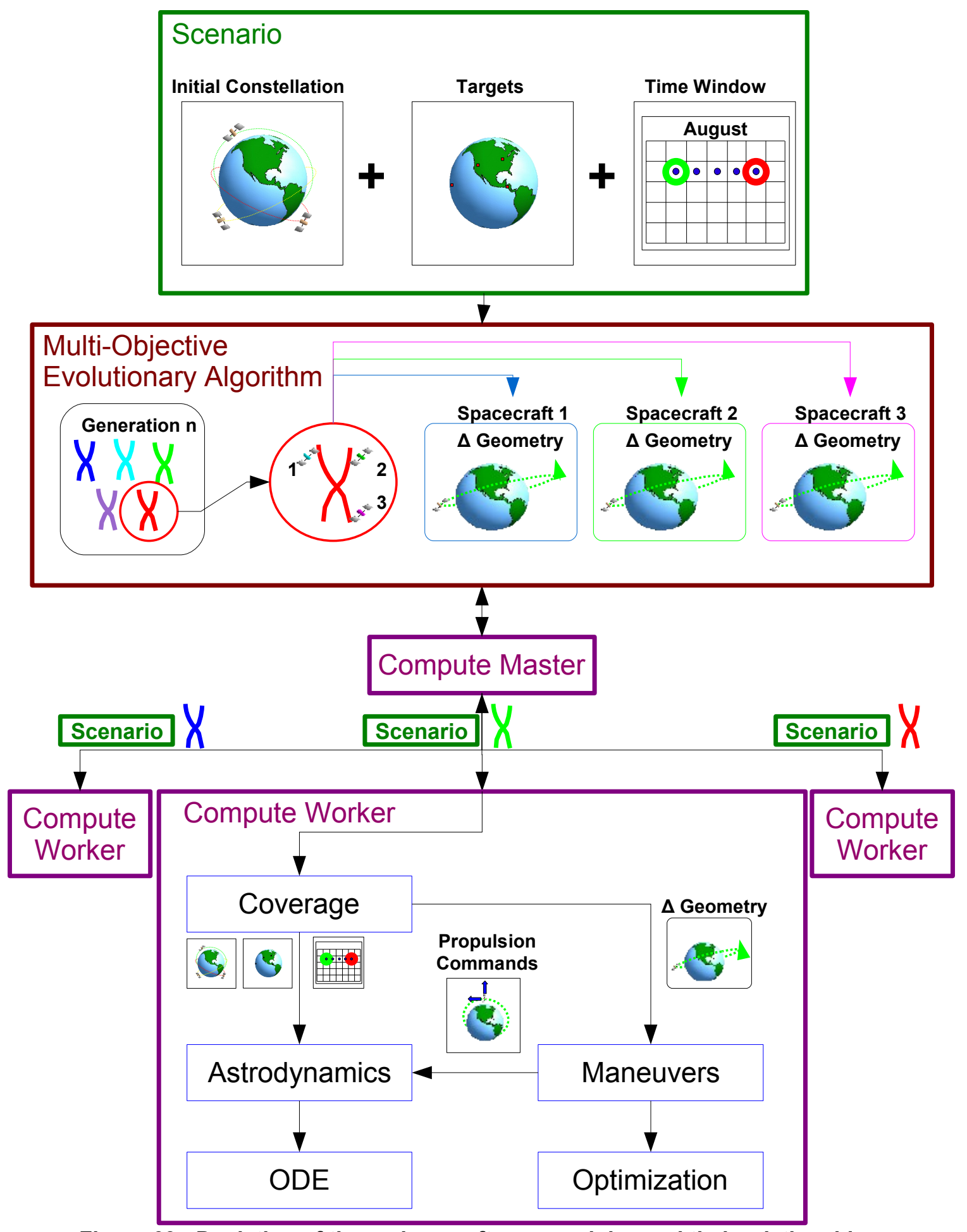

Figure 63: Depiction of the various software modules and their relationships. 


\section{Appendix C: Supplemental Data for Chapter 6: Examples}

\begin{tabular}{|c|c|c|c|c|c|}
\hline Solution & Vehicle & Maneuver & $\begin{array}{c}\text { Propellant } \\
\text { Consumed by } \\
\text { Constellation } \\
{[\mathrm{kg}]}\end{array}$ & $\begin{array}{c}\text { Total Coverage } \\
\text { Time [hr] } \\
\text { Washington, } \\
\text { D.C. }\end{array}$ & $\begin{array}{c}\text { Total Coverage } \\
\text { Time [hr] } \\
\text { Beijing }\end{array}$ \\
\hline 1 & 1 & SMA Maximizer - Initial Peri.: 111.7 [deg] & 9.8 & 10.9 & 9.7 \\
\hline 2 & 1 & SMA and Ecc. Maximizer - Initial Peri.: 129.2 [deg] & 9.6 & 10.8 & 9.4 \\
\hline 3 & 1 & SMA Maximizer - Initial Peri.: 125.1 [deg] & 9.6 & 10.8 & 9.5 \\
\hline 4 & 1 & SMA and Ecc. Maximizer - Initial Peri.: 117.9 [deg] & 9.6 & 10.7 & 9.5 \\
\hline 5 & 1 & SMA and Ecc. Maximizer - Initial Peri.: 126.9 [deg] & 9.5 & 10.7 & 9.2 \\
\hline 6 & 1 & SMA Maximizer - Initial Peri.: 142.5 [deg] & 9.4 & 10.5 & 9.0 \\
\hline 7 & 1 & SMA and Ecc. Maximizer - Initial Peri.: 118.1 [deg] & 9.4 & 10.4 & 9.2 \\
\hline 8 & 1 & SMA and Ecc. Maximizer - Initial Peri.: 114.6 [deg] & 9.4 & 10.4 & 9.3 \\
\hline 9 & 1 & SMA and Ecc. Maximizer - Initial Peri.: 126.5 [deg] & 9.2 & 10.3 & 8.9 \\
\hline 10 & 1 & SMA and Ecc. Maximizer - Initial Peri.: 109.9 [deg] & 9.3 & 10.2 & 9.1 \\
\hline 11 & 1 & SMA and Ecc. Maximizer - Initial Peri.: 135.9 [deg] & 9.1 & 10.1 & 8.7 \\
\hline 12 & 1 & SMA and Ecc. Maximizer - Initial Peri.: 109.3 [deg] & 9.1 & 10.1 & 8.9 \\
\hline 13 & 1 & SMA and Ecc. Maximizer - Initial Peri.: 126.5 [deg] & 8.9 & 9.9 & 8.5 \\
\hline 14 & 1 & SMA Maximizer - Initial Peri.: 125.6 [deg] & 8.9 & 9.9 & 8.5 \\
\hline 15 & 1 & SMA Maximizer - Initial Peri.: 107.8 [deg] & 9.0 & 9.8 & 8.8 \\
\hline 16 & 1 & SMA Maximizer - Initial Peri.: 114.6 [deg] & 8.9 & 9.8 & 8.6 \\
\hline 17 & 1 & SMA Maximizer - Initial Peri.: 131.2 [deg] & 8.7 & 9.8 & 8.3 \\
\hline 18 & 1 & SMA Maximizer - Initial Peri.: 129.2 [deg] & 8.7 & 9.8 & 8.4 \\
\hline 19 & 1 & SMA Maximizer - Initial Peri.: 109.3 [deg] & 8.9 & 9.7 & 8.6 \\
\hline
\end{tabular}




\begin{tabular}{|c|c|c|c|c|c|}
\hline Solution & Vehicle & Maneuver & $\begin{array}{c}\text { Propellant } \\
\text { Consumed by } \\
\text { Constellation } \\
{[\mathrm{kg}]}\end{array}$ & $\begin{array}{c}\text { Total Coverage } \\
\text { Time [hr] } \\
\text { Washington, } \\
\text { D.C. }\end{array}$ & $\begin{array}{l}\text { Total Coverage } \\
\text { Time [hr] } \\
\text { Beijing }\end{array}$ \\
\hline 20 & 1 & SMA Maximizer - Initial Peri.: 132.3 [deg] & 8.7 & 9.6 & 8.3 \\
\hline 21 & 1 & SMA Maximizer - Initial Peri.: 113.1 [deg] & 8.6 & 9.4 & 8.2 \\
\hline 22 & 1 & SMA Maximizer - Initial Peri.: 125.1 [deg] & 8.3 & 9.2 & 7.9 \\
\hline 23 & 1 & SMA Maximizer - Initial Peri.: 129.7 [deg] & 8.2 & 9.1 & 7.6 \\
\hline 24 & 1 & SMA and Ecc. Maximizer - Initial Peri.: 101.1 [deg] & 8.4 & 9.1 & 7.9 \\
\hline 25 & 1 & SMA Maximizer - Initial Peri.: 127.4 [deg] & 8.2 & 9.0 & 7.7 \\
\hline 26 & 1 & SMA Maximizer - Initial Peri.: 125.6 [deg] & 8.1 & 8.9 & 7.6 \\
\hline 27 & 1 & SMA Maximizer - Initial Peri.: 109.3 [deg] & 8.2 & 8.9 & 7.7 \\
\hline 28 & 1 & SMA and Ecc. Maximizer - Initial Peri.: 115.7 [deg] & 7.9 & 8.7 & 7.2 \\
\hline 29 & 1 & SMA Maximizer - Initial Peri.: 124.4 [deg] & 7.9 & 8.7 & 7.2 \\
\hline 30 & 1 & SMA Maximizer - Initial Peri.: 100.5 [deg] & 8.1 & 8.6 & 7.5 \\
\hline 31 & 1 & SMA Maximizer - Initial Peri.: 115.2 [deg] & 7.8 & 8.6 & 7.0 \\
\hline 32 & 1 & SMA Maximizer - Initial Peri.: 111.7 [deg] & 7.9 & 8.5 & 7.4 \\
\hline 33 & 1 & SMA Maximizer - Initial Peri.: 104.6 [deg] & 7.8 & 8.5 & 7.0 \\
\hline 34 & 1 & SMA Maximizer - Initial Peri.: 112.6 [deg] & 7.7 & 8.5 & 7.1 \\
\hline 35 & 1 & SMA Maximizer - Initial Peri.: 95.5 [deg] & 7.7 & 8.3 & 6.9 \\
\hline 36 & 1 & SMA Maximizer - Initial Peri.: 116.5 [deg] & 7.5 & 8.0 & 6.7 \\
\hline 37 & 1 & SMA Maximizer - Initial Peri.: 118.2 [deg] & 7.6 & 7.9 & 6.8 \\
\hline 38 & 1 & SMA and Ecc. Maximizer - Initial Peri.: 113.2 [deg] & 7.6 & 7.9 & 6.8 \\
\hline 39 & 1 & SMA Maximizer - Initial Peri.: 94.1 [deg] & 7.4 & 7.7 & 6.3 \\
\hline 40 & 1 & SMA Maximizer - Initial Peri.: 123.6 [deg] & 6.9 & 7.6 & 6.0 \\
\hline 41 & 1 & SMA and Ecc. Maximizer - Initial Peri.: 118.1 [deg] & 6.9 & 7.4 & 6.1 \\
\hline
\end{tabular}




\begin{tabular}{|c|c|c|c|c|c|}
\hline Solution & Vehicle & Maneuver & $\begin{array}{c}\text { Propellant } \\
\text { Consumed by } \\
\text { Constellation } \\
{[\mathrm{kg}]}\end{array}$ & $\begin{array}{c}\text { Total Coverage } \\
\text { Time [hr] } \\
\text { Washington, } \\
\text { D.C. }\end{array}$ & $\begin{array}{l}\text { Total Coverage } \\
\text { Time [hr] } \\
\text { Beijing }\end{array}$ \\
\hline 42 & 1 & SMA Maximizer - Initial Peri.: 156.0 [deg] & 6.9 & 7.3 & 5.7 \\
\hline 43 & 1 & SMA Maximizer - Initial Peri.: 116.5 [deg] & 6.9 & 7.2 & 6.2 \\
\hline 44 & 1 & SMA Maximizer - Initial Peri.: 143.1 [deg] & 6.8 & 7.1 & 6.0 \\
\hline 45 & 1 & SMA and Ecc. Maximizer - Initial Peri.: 135.9 [deg] & 6.6 & 7.0 & 5.8 \\
\hline 46 & 1 & SMA Maximizer - Initial Peri.: 135.9 [deg] & 6.6 & 7.0 & 5.8 \\
\hline 47 & 1 & SMA and Ecc. Maximizer - Initial Peri.: 125.6 [deg] & 6.8 & 7.0 & 6.2 \\
\hline 48 & 1 & SMA Maximizer - Initial Peri.: 143.3 [deg] & 6.5 & 6.9 & 5.5 \\
\hline 49 & 1 & SMA Maximizer - Initial Peri.: 142.8 [deg] & 6.5 & 6.8 & 5.5 \\
\hline 50 & 1 & SMA Maximizer - Initial Peri.: 125.6 [deg] & 6.5 & 6.8 & 5.6 \\
\hline 51 & 1 & SMA and Ecc. Maximizer - Initial Peri.: 95.5 [deg] & 6.4 & 6.7 & 5.4 \\
\hline 52 & 1 & SMA Maximizer - Initial Peri.: 129.3 [deg] & 6.3 & 6.6 & 5.6 \\
\hline 53 & 1 & SMA Maximizer - Initial Peri.: 113.1 [deg] & 6.3 & 6.6 & 5.4 \\
\hline 54 & 1 & SMA Maximizer - Initial Peri.: 111.7 [deg] & 6.2 & 6.4 & 5.2 \\
\hline 55 & 1 & SMA and Ecc. Maximizer - Initial Peri.: 107.4 [deg] & 6.1 & 6.3 & 5.1 \\
\hline 56 & 1 & SMA and Ecc. Maximizer - Initial Peri.: 116.5 [deg] & 6.1 & 6.3 & 5.2 \\
\hline 57 & 1 & SMA Maximizer - Initial Peri.: 123.6 [deg] & 5.9 & 6.2 & 4.9 \\
\hline 58 & 1 & SMA Maximizer - Initial Peri.: 129.7 [deg] & 5.9 & 6.2 & 5.0 \\
\hline 59 & 1 & SMA and Ecc. Maximizer - Initial Peri.: 122.9 [deg] & 5.8 & 6.0 & 4.7 \\
\hline 60 & 1 & SMA Maximizer - Initial Peri.: 107.4 [deg] & 5.8 & 5.9 & 4.8 \\
\hline 61 & 1 & SMA and Ecc. Maximizer - Initial Peri.: 118.1 [deg] & 5.7 & 5.9 & 4.7 \\
\hline 62 & 1 & SMA and Ecc. Maximizer - Initial Peri.: 119.7 [deg] & 5.6 & 5.8 & 4.6 \\
\hline 63 & 1 & SMA Maximizer - Initial Peri.: 145.2 [deg] & 5.6 & 5.8 & 4.5 \\
\hline
\end{tabular}




\begin{tabular}{|c|c|c|c|c|c|}
\hline Solution & Vehicle & Maneuver & $\begin{array}{c}\text { Propellant } \\
\text { Consumed by } \\
\text { Constellation } \\
{[\mathrm{kg}]}\end{array}$ & $\begin{array}{c}\text { Total Coverage } \\
\text { Time [hr] } \\
\text { Washington, } \\
\text { D.C. }\end{array}$ & $\begin{array}{l}\text { Total Coverage } \\
\text { Time [hr] } \\
\text { Beijing }\end{array}$ \\
\hline 64 & 1 & SMA Maximizer - Initial Peri.: 123.6 [deg] & 5.3 & 5.6 & 4.3 \\
\hline 65 & 1 & SMA Maximizer - Initial Peri.: 125.6 [deg] & 5.3 & 5.5 & 4.4 \\
\hline 66 & 1 & SMA Maximizer - Initial Peri.: 121.4 [deg] & 5.3 & 5.5 & 4.3 \\
\hline 67 & 1 & SMA and Ecc. Maximizer - Initial Peri.: 125.1 [deg] & 5.2 & 5.4 & 4.1 \\
\hline 68 & 1 & SMA Maximizer - Initial Peri.: 123.6 [deg] & 5.1 & 5.3 & 4.0 \\
\hline 69 & 1 & SMA Maximizer - Initial Peri.: 114.3 [deg] & 5.0 & 5.3 & 4.0 \\
\hline 70 & 1 & SMA and Ecc. Maximizer - Initial Peri.: 109.9 [deg] & 5.0 & 5.2 & 4.0 \\
\hline 71 & 1 & SMA Maximizer - Initial Peri.: 84.2 [deg] & 5.0 & 4.9 & 3.7 \\
\hline 72 & 1 & SMA Maximizer - Initial Peri.: 120.5 [deg] & 4.8 & 4.9 & 3.7 \\
\hline 73 & 1 & SMA Maximizer - Initial Peri.: 108.4 [deg] & 4.7 & 4.9 & 3.7 \\
\hline 74 & 1 & SMA Maximizer - Initial Peri.: 122.5 [deg] & 4.5 & 4.7 & 3.5 \\
\hline 75 & 1 & SMA Maximizer - Initial Peri.: 109.9 [deg] & 4.6 & 4.6 & 3.5 \\
\hline 76 & 1 & SMA Maximizer - Initial Peri.: 132.9 [deg] & 4.5 & 4.6 & 3.4 \\
\hline 77 & 1 & SMA and Ecc. Maximizer - Initial Peri.: 97.9 [deg] & 4.4 & 4.4 & 3.3 \\
\hline 78 & 1 & SMA Maximizer - Initial Peri.: 104.7 [deg] & 4.0 & 4.1 & 2.9 \\
\hline 79 & 1 & SMA and Ecc. Maximizer - Initial Peri.: 106.2 [deg] & 4.0 & 4.0 & 2.8 \\
\hline 80 & 1 & SMA Maximizer - Initial Peri.: 152.1 [deg] & 3.9 & 4.0 & 2.7 \\
\hline 81 & 1 & SMA and Ecc. Maximizer - Initial Peri.: 125.6 [deg] & 3.8 & 3.9 & 2.6 \\
\hline 82 & 1 & SMA and Ecc. Maximizer - Initial Peri.: 126.9 [deg] & 3.8 & 3.8 & 2.5 \\
\hline 83 & 1 & SMA Maximizer - Initial Peri.: 127.6 [deg] & 3.8 & 3.8 & 2.6 \\
\hline 84 & 1 & SMA Maximizer - Initial Peri.: 109.9 [deg] & 3.7 & 3.8 & 2.5 \\
\hline 85 & 1 & SMA and Ecc. Maximizer - Initial Peri.: 143.3 [deg] & 3.6 & 3.7 & 2.3 \\
\hline
\end{tabular}




\begin{tabular}{|c|c|c|c|c|c|}
\hline Solution & Vehicle & Maneuver & $\begin{array}{c}\text { Propellant } \\
\text { Consumed by } \\
\text { Constellation } \\
{[\mathrm{kg}]}\end{array}$ & $\begin{array}{c}\text { Total Coverage } \\
\text { Time [hr] } \\
\text { Washington, } \\
\text { D.C. }\end{array}$ & $\begin{array}{l}\text { Total Coverage } \\
\text { Time [hr] } \\
\text { Beijing }\end{array}$ \\
\hline 86 & 1 & SMA Maximizer - Initial Peri.: 113.1 [deg] & 3.5 & 3.5 & 2.3 \\
\hline 87 & 1 & SMA Maximizer - Initial Peri.: 126.9 [deg] & 3.3 & 3.4 & 2.2 \\
\hline 88 & 1 & SMA Maximizer - Initial Peri.: 107.8 [deg] & 3.3 & 3.3 & 2.1 \\
\hline 89 & 1 & SMA Maximizer - Initial Peri.: 125.6 [deg] & 3.2 & 3.2 & 2.0 \\
\hline 90 & 1 & SMA Maximizer - Initial Peri.: 127.6 [deg] & 3.1 & 3.1 & 2.0 \\
\hline 91 & 1 & SMA and Ecc. Maximizer - Initial Peri.: 147.8 [deg] & 3.0 & 3.0 & 1.8 \\
\hline 92 & 1 & SMA Maximizer - Initial Peri.: 99.7 [deg] & 2.8 & 2.9 & 1.7 \\
\hline 93 & 1 & SMA and Ecc. Maximizer - Initial Peri.: 114.6 [deg] & 2.7 & 2.8 & 1.6 \\
\hline 94 & 1 & SMA Maximizer - Initial Peri.: 126.9 [deg] & 2.5 & 2.6 & 1.4 \\
\hline 95 & 1 & SMA Maximizer - Initial Peri.: 113.1 [deg] & 2.6 & 2.6 & 1.4 \\
\hline 96 & 1 & SMA Maximizer - Initial Peri.: 127.6 [deg] & 2.5 & 2.6 & 1.5 \\
\hline 97 & 1 & SMA and Ecc. Maximizer - Initial Peri.: 112.8 [deg] & 2.5 & 2.5 & 1.4 \\
\hline 98 & 1 & SMA and Ecc. Maximizer - Initial Peri.: 127.6 [deg] & 2.4 & 2.5 & 1.3 \\
\hline 99 & 1 & SMA and Ecc. Maximizer - Initial Peri.: 108.0 [deg] & 2.2 & 2.3 & 1.2 \\
\hline 100 & 1 & SMA and Ecc. Maximizer - Initial Peri.: 107.4 [deg] & 2.1 & 2.2 & 1.1 \\
\hline 101 & 1 & SMA and Ecc. Maximizer - Initial Peri.: 118.1 [deg] & 2.0 & 2.1 & 1.0 \\
\hline 102 & 1 & SMA and Ecc. Maximizer - Initial Peri.: 129.2 [deg] & 2.0 & 2.0 & 0.9 \\
\hline 103 & 1 & SMA and Ecc. Maximizer - Initial Peri.: 113.1 [deg] & 1.8 & 1.9 & 0.8 \\
\hline 104 & 1 & SMA and Ecc. Maximizer - Initial Peri.: 109.9 [deg] & 1.7 & 1.8 & 0.7 \\
\hline 105 & 1 & SMA and Ecc. Maximizer - Initial Peri.: 206.3 [deg] & 1.9 & 1.7 & 0.8 \\
\hline 106 & 1 & SMA Maximizer - Initial Peri.: 109.3 [deg] & 1.6 & 1.7 & 0.6 \\
\hline 107 & 1 & SMA and Ecc. Maximizer - Initial Peri.: 113.1 [deg] & 1.5 & 1.7 & 0.6 \\
\hline
\end{tabular}




\begin{tabular}{|c|c|c|c|c|c|}
\hline Solution & Vehicle & Maneuver & $\begin{array}{c}\text { Propellant } \\
\text { Consumed by } \\
\text { Constellation } \\
{[\mathrm{kg}]}\end{array}$ & $\begin{array}{c}\text { Total Coverage } \\
\text { Time [hr] } \\
\text { Washington, } \\
\text { D.C. }\end{array}$ & $\begin{array}{c}\text { Total Coverage } \\
\text { Time [hr] } \\
\text { Beijing }\end{array}$ \\
\hline 108 & 1 & SMA and Ecc. Maximizer - Initial Peri.: 125.6 [deg] & 1.3 & 1.5 & 0.5 \\
\hline 109 & 1 & SMA and Ecc. Maximizer - Initial Peri.: 133.0 [deg] & 1.2 & 1.3 & 0.3 \\
\hline 110 & 1 & SMA and Ecc. Maximizer - Initial Peri.: 112.6 [deg] & 1.1 & 1.3 & 0.3 \\
\hline 111 & 1 & SMA and Ecc. Maximizer - Initial Peri.: 114.6 [deg] & 1.0 & 1.2 & 0.2 \\
\hline 112 & 1 & SMA Maximizer - Initial Peri.: 79.2 [deg] & 1.0 & 1.1 & 0.2 \\
\hline 113 & 1 & SMA Maximizer - Initial Peri.: 125.3 [deg] & 0.9 & 1.1 & 0.1 \\
\hline 114 & 1 & SMA Maximizer - Initial Peri.: 101.9 [deg] & 0.8 & 1.0 & 0.1 \\
\hline 115 & 1 & SMA Maximizer - Initial Peri.: 144.7 [deg] & 0.7 & 0.9 & 0.0 \\
\hline 116 & 1 & SMA Maximizer - Initial Peri.: 73.9 [deg] & 0.6 & 0.8 & 0.0 \\
\hline 117 & 1 & SMA Maximizer - Initial Peri.: 49.9 [deg] & 0.4 & 0.7 & 0.0 \\
\hline 118 & 1 & SMA Maximizer - Initial Peri.: 125.6 [deg] & 0.3 & 0.6 & 0.0 \\
\hline 119 & 1 & SMA Maximizer w/Constant Ecc. & 0.3 & 0.6 & 0.0 \\
\hline 120 & 1 & SMA Maximizer - Initial Peri.: 129.2 [deg] & 0.2 & 0.6 & 0.0 \\
\hline 121 & 1 & SMA Maximizer - Initial Peri.: 144.7 [deg] & 0.1 & 0.5 & 0.0 \\
\hline 122 & 1 & Zero maneuver & 0.0 & 0.4 & 0.0 \\
\hline
\end{tabular}


Table 18: Final nondominated surface for Example 6.2

\begin{tabular}{|c|c|c|c|c|c|}
\hline Solution & Vehicle & Maneuver & \begin{tabular}{|c|} 
Propellant \\
Consumed by \\
Constellation \\
{$[\mathrm{kg}]$}
\end{tabular} & $\begin{array}{c}\text { Total Coverage } \\
\text { Time [hr] } \\
\text { Washington, } \\
\text { D.C. }\end{array}$ & $\begin{array}{c}\text { Total Coverage } \\
\text { Time [hr] } \\
\text { Montevideo }\end{array}$ \\
\hline 1 & 1 & SMA Maximizer - Initial Peri.: 128.3 [deg] & 10.0 & 11.3 & 8.8 \\
\hline 2 & 1 & SMA Maximizer - Initial Peri.: 141.7 [deg] & 10.0 & 11.2 & 9.0 \\
\hline 3 & 1 & SMA Maximizer - Initial Peri.: 121.0 [deg] & 9.9 & 11.0 & 8.8 \\
\hline 4 & 1 & SMA Maximizer - Initial Peri.: 153.2 [deg] & 10.0 & 10.8 & 9.3 \\
\hline 5 & 1 & SMA Maximizer - Initial Peri.: 160.2 [deg] & 10.0 & 10.5 & 9.5 \\
\hline 6 & 1 & SMA Maximizer - Initial Peri.: 146.5 [deg] & 9.5 & 10.4 & 8.9 \\
\hline 7 & 1 & SMA Maximizer - Initial Peri.: 128.3 [deg] & 9.1 & 10.1 & 8.6 \\
\hline 8 & 1 & SMA Maximizer - Initial Peri.: 157.4 [deg] & 9.3 & 9.9 & 9.2 \\
\hline 9 & 1 & SMA Maximizer - Initial Peri.: 167.6 [deg] & 9.7 & 9.8 & 9.7 \\
\hline 10 & 1 & SMA Maximizer - Initial Peri.: 116.8 [deg] & 8.8 & 9.7 & 8.6 \\
\hline 11 & 1 & SMA Maximizer - Initial Peri.: 121.9 [deg] & 8.6 & 9.5 & 8.6 \\
\hline 12 & 1 & SMA Maximizer - Initial Peri.: 181.1 [deg] & 9.9 & 9.4 & 10.6 \\
\hline 13 & 1 & SMA Maximizer - Initial Peri.: 177.1 [deg] & 9.6 & 9.3 & 10.2 \\
\hline 14 & 1 & SMA and Ecc. Maximizer - Initial Peri.: 74.5 [deg] & 9.1 & 9.3 & 9.5 \\
\hline 15 & 1 & SMA Maximizer - Initial Peri.: 77.6 [deg] & 8.9 & 9.1 & 9.3 \\
\hline 16 & 1 & SMA Maximizer - Initial Peri.: 79.5 [deg] & 8.8 & 9.0 & 9.2 \\
\hline 17 & 1 & SMA Maximizer - Initial Peri.: 166.4 [deg] & 8.8 & 8.9 & 9.3 \\
\hline 18 & 1 & SMA and Ecc. Maximizer - Initial Peri.: 185.1 [deg] & 9.5 & 8.9 & 10.7 \\
\hline 19 & 1 & SMA and Ecc. Maximizer - Initial Peri.: 188.1 [deg] & 9.6 & 8.8 & 10.8 \\
\hline 20 & 1 & SMA Maximizer - Initial Peri.: 79.5 [deg] & 8.5 & 8.7 & 9.0 \\
\hline 21 & 1 & SMA and Ecc. Maximizer - Initial Peri.: 151.1 [deg] & 8.1 & 8.7 & 8.7 \\
\hline
\end{tabular}




\begin{tabular}{|c|c|c|c|c|c|}
\hline Solution & Vehicle & Maneuver & $\begin{array}{c}\text { Propellant } \\
\text { Consumed by } \\
\text { Constellation } \\
{[\mathrm{kg}]}\end{array}$ & $\begin{array}{c}\text { Total Coverage } \\
\text { Time [hr] } \\
\text { Washington, } \\
\text { D.C. }\end{array}$ & $\begin{array}{c}\text { Total Coverage } \\
\text { Time [hr] } \\
\text { Montevideo }\end{array}$ \\
\hline 22 & 1 & SMA Maximizer - Initial Peri.: 70.2 [deg] & 8.6 & 8.6 & 9.4 \\
\hline 23 & 1 & SMA Maximizer - Initial Peri.: 160.2 [deg] & 8.2 & 8.5 & 8.9 \\
\hline 24 & 1 & SMA Maximizer - Initial Peri.: 193.9 [deg] & 9.5 & 8.4 & 11.2 \\
\hline 25 & 1 & SMA Maximizer - Initial Peri.: 196.5 [deg] & 9.7 & 8.4 & 11.4 \\
\hline 26 & 1 & SMA Maximizer - Initial Peri.: 197.6 [deg] & 9.5 & 8.2 & 11.4 \\
\hline 27 & 1 & SMA Maximizer - Initial Peri.: 166.4 [deg] & 7.9 & 8.1 & 9.0 \\
\hline 28 & 1 & SMA and Ecc. Maximizer - Initial Peri.: 198.2 [deg] & 9.3 & 8.0 & 11.3 \\
\hline 29 & 1 & SMA Maximizer - Initial Peri.: 181.4 [deg] & 8.4 & 8.0 & 9.8 \\
\hline 30 & 1 & SMA Maximizer - Initial Peri.: 139.3 [deg] & 7.6 & 8.0 & 8.3 \\
\hline 31 & 1 & SMA Maximizer - Initial Peri.: 104.8 [deg] & 7.4 & 7.9 & 8.3 \\
\hline 32 & 1 & SMA Maximizer - Initial Peri.: 126.1 [deg] & 7.2 & 7.7 & 8.4 \\
\hline 33 & 1 & SMA Maximizer - Initial Peri.: 102.0 [deg] & 7.2 & 7.7 & 8.4 \\
\hline 34 & 1 & SMA Maximizer w/Constant Ecc. & 9.7 & 7.5 & 12.7 \\
\hline 35 & 1 & SMA Maximizer - Initial Peri.: 26.5 [deg] & 9.3 & 7.5 & 11.6 \\
\hline 36 & 1 & SMA Maximizer w/Constant Ecc. & 9.6 & 7.5 & 12.5 \\
\hline 37 & 1 & SMA Maximizer - Initial Peri.: 214.0 [deg] & 10.0 & 7.4 & 12.8 \\
\hline 38 & 1 & SMA Maximizer w/Constant Ecc. & 9.4 & 7.3 & 12.4 \\
\hline 39 & 1 & SMA Maximizer w/Constant Ecc. & 9.0 & 7.1 & 12.1 \\
\hline 40 & 1 & SMA Maximizer - Initial Peri.: 220.2 [deg] & 10.0 & 7.0 & 13.3 \\
\hline 41 & 1 & SMA Maximizer w/Constant Ecc. & 8.8 & 6.9 & 11.9 \\
\hline 42 & 1 & SMA and Ecc. Maximizer - Initial Peri.: 215.3 [deg] & 9.2 & 6.9 & 12.3 \\
\hline 43 & 1 & SMA Maximizer - Initial Peri.: 130.9 [deg] & 6.4 & 6.8 & 8.1 \\
\hline
\end{tabular}




\begin{tabular}{|c|c|c|c|c|c|}
\hline Solution & Vehicle & Maneuver & $\begin{array}{c}\text { Propellant } \\
\text { Consumed by } \\
\text { Constellation } \\
{[\mathrm{kg}]}\end{array}$ & $\begin{array}{c}\text { Total Coverage } \\
\text { Time [hr] } \\
\text { Washington, } \\
\text { D.C. }\end{array}$ & $\begin{array}{c}\text { Total Coverage } \\
\text { Time [hr] } \\
\text { Montevideo }\end{array}$ \\
\hline 44 & 1 & SMA and Ecc. Maximizer - Initial Peri.: 163.4 [deg] & 6.5 & 6.7 & 8.4 \\
\hline 45 & 1 & SMA Maximizer - Initial Peri.: 226.2 [deg] & 10.0 & 6.6 & 13.7 \\
\hline 46 & 1 & SMA Maximizer w/Constant Ecc. & 8.4 & 6.4 & 11.5 \\
\hline 47 & 1 & SMA Maximizer - Initial Peri.: 219.2 [deg] & 8.8 & 6.4 & 12.2 \\
\hline 48 & 1 & SMA Maximizer - Initial Peri.: 201.1 [deg] & 7.9 & 6.4 & 10.6 \\
\hline 49 & 1 & SMA Maximizer - Initial Peri.: 197.7 [deg] & 7.2 & 6.3 & 9.9 \\
\hline 50 & 1 & SMA Maximizer - Initial Peri.: 122.2 [deg] & 6.1 & 6.2 & 7.8 \\
\hline 51 & 1 & SMA Maximizer - Initial Peri.: 1.5 [deg] & 9.4 & 6.1 & 13.2 \\
\hline 52 & 1 & SMA and Ecc. Maximizer - Initial Peri.: 26.5 [deg] & 7.5 & 6.1 & 10.0 \\
\hline 53 & 1 & SMA Maximizer - Initial Peri.: 229.8 [deg] & 9.1 & 6.0 & 13.0 \\
\hline 54 & 1 & SMA Maximizer - Initial Peri.: 155.1 [deg] & 5.9 & 5.9 & 8.0 \\
\hline 55 & 1 & SMA Maximizer - Initial Peri.: 128.5 [deg] & 5.6 & 5.8 & 7.6 \\
\hline 56 & 1 & SMA Maximizer w/Constant Ecc. & 7.2 & 5.7 & 10.0 \\
\hline 57 & 1 & SMA Maximizer w/Constant Ecc. & 7.5 & 5.6 & 10.9 \\
\hline 58 & 1 & SMA and Ecc. Maximizer - Initial Peri.: 71.6 [deg] & 5.8 & 5.5 & 8.2 \\
\hline 59 & 1 & SMA Maximizer - Initial Peri.: 228.6 [deg] & 8.2 & 5.5 & 12.1 \\
\hline 60 & 1 & SMA Maximizer w/Constant Ecc. & 7.0 & 5.4 & 10.4 \\
\hline 61 & 1 & SMA and Ecc. Maximizer - Initial Peri.: 245.1 [deg] & 9.9 & 5.3 & 14.8 \\
\hline 62 & 1 & SMA Maximizer - Initial Peri.: 247.5 [deg] & 9.9 & 5.3 & 14.8 \\
\hline 63 & 1 & SMA Maximizer w/Constant Ecc. & 6.7 & 5.2 & 10.2 \\
\hline 64 & 1 & SMA and Ecc. Maximizer - Initial Peri.: 239.1 [deg] & 8.3 & 5.1 & 12.7 \\
\hline 65 & 1 & SMA and Ecc. Maximizer - Initial Peri.: 228.6 [deg] & 7.5 & 5.0 & 11.7 \\
\hline
\end{tabular}




\begin{tabular}{|c|c|c|c|c|c|}
\hline Solution & Vehicle & Maneuver & $\begin{array}{c}\text { Propellant } \\
\text { Consumed by } \\
\text { Constellation } \\
{[\mathrm{kg}]}\end{array}$ & $\begin{array}{c}\text { Total Coverage } \\
\text { Time [hr] } \\
\text { Washington, } \\
\text { D.C. }\end{array}$ & $\begin{array}{c}\text { Total Coverage } \\
\text { Time [hr] } \\
\text { Montevideo }\end{array}$ \\
\hline 66 & 1 & SMA and Ecc. Maximizer - Initial Peri.: 334.3 [deg] & 10.0 & 4.9 & 15.3 \\
\hline 67 & 1 & SMA Maximizer - Initial Peri.: 92.6 [deg] & 4.8 & 4.8 & 7.4 \\
\hline 68 & 1 & SMA and Ecc. Maximizer - Initial Peri.: 172.8 [deg] & 4.9 & 4.7 & 7.8 \\
\hline 69 & 1 & SMA and Ecc. Maximizer - Initial Peri.: 128.3 [deg] & 4.5 & 4.7 & 7.1 \\
\hline 70 & 1 & SMA Maximizer - Initial Peri.: 256.8 [deg] & 9.3 & 4.6 & 14.6 \\
\hline 71 & 1 & SMA Maximizer - Initial Peri.: 258.2 [deg] & 9.2 & 4.5 & 14.6 \\
\hline 72 & 1 & SMA and Ecc. Maximizer - Initial Peri.: 214.3 [deg] & 5.7 & 4.4 & 9.4 \\
\hline 73 & 1 & SMA Maximizer - Initial Peri.: 193.5 [deg] & 4.8 & 4.3 & 8.1 \\
\hline 74 & 1 & SMA and Ecc. Maximizer - Initial Peri.: 234.2 [deg] & 6.1 & 4.2 & 10.2 \\
\hline 75 & 1 & SMA Maximizer - Initial Peri.: 317.8 [deg] & 9.6 & 4.2 & 15.6 \\
\hline 76 & 1 & SMA Maximizer - Initial Peri.: 324.4 [deg] & 8.5 & 4.1 & 14.0 \\
\hline 77 & 1 & SMA Maximizer - Initial Peri.: 272.0 [deg] & 9.2 & 4.0 & 15.1 \\
\hline 78 & 1 & SMA and Ecc. Maximizer - Initial Peri.: 276.8 [deg] & 9.6 & 4.0 & 15.7 \\
\hline 79 & 1 & SMA Maximizer - Initial Peri.: 305.0 [deg] & 10.0 & 4.0 & 16.3 \\
\hline 80 & 1 & SMA and Ecc. Maximizer - Initial Peri.: 307.9 [deg] & 9.6 & 3.9 & 15.9 \\
\hline 81 & 1 & SMA Maximizer w/Constant Ecc. & 5.0 & 3.9 & 8.7 \\
\hline 82 & 1 & SMA Maximizer - Initial Peri.: 37.0 [deg] & 4.5 & 3.9 & 8.0 \\
\hline 83 & 1 & SMA Maximizer - Initial Peri.: 305.6 [deg] & 9.4 & 3.8 & 15.5 \\
\hline 84 & 1 & SMA Maximizer - Initial Peri.: 305.0 [deg] & 9.3 & 3.8 & 15.4 \\
\hline 85 & 1 & SMA Maximizer - Initial Peri.: 264.6 [deg] & 7.5 & 3.8 & 12.9 \\
\hline 86 & 1 & SMA Maximizer - Initial Peri.: 291.3 [deg] & 10.0 & 3.8 & 16.3 \\
\hline 87 & 1 & SMA Maximizer - Initial Peri.: 288.2 [deg] & 9.8 & 3.7 & 16.1 \\
\hline
\end{tabular}




\begin{tabular}{|c|c|c|c|c|c|}
\hline Solution & Vehicle & Maneuver & $\begin{array}{c}\text { Propellant } \\
\text { Consumed by } \\
\text { Constellation } \\
{[\mathrm{kg}]}\end{array}$ & $\begin{array}{c}\text { Total Coverage } \\
\text { Time [hr] } \\
\text { Washington, } \\
\text { D.C. }\end{array}$ & $\begin{array}{c}\text { Total Coverage } \\
\text { Time [hr] } \\
\text { Montevideo }\end{array}$ \\
\hline 88 & 1 & SMA and Ecc. Maximizer - Initial Peri.: 198.8 [deg] & 4.2 & 3.6 & 7.8 \\
\hline 89 & 1 & SMA and Ecc. Maximizer - Initial Peri.: 182.7 [deg] & 3.8 & 3.6 & 7.3 \\
\hline 90 & 1 & SMA Maximizer - Initial Peri.: 288.2 [deg] & 7.9 & 3.5 & 13.7 \\
\hline 91 & 1 & SMA Maximizer - Initial Peri.: 273.6 [deg] & 7.0 & 3.5 & 12.0 \\
\hline 92 & 1 & SMA Maximizer - Initial Peri.: 329.2 [deg] & 6.2 & 3.5 & 11.1 \\
\hline 93 & 1 & SMA Maximizer - Initial Peri.: 280.9 [deg] & 7.8 & 3.5 & 13.5 \\
\hline 94 & 1 & SMA Maximizer - Initial Peri.: 198.2 [deg] & 4.0 & 3.4 & 7.7 \\
\hline 95 & 1 & SMA Maximizer - Initial Peri.: 275.0 [deg] & 6.4 & 3.3 & 11.6 \\
\hline 96 & 1 & SMA Maximizer - Initial Peri.: 63.2 [deg] & 3.4 & 3.3 & 6.9 \\
\hline 97 & 1 & SMA and Ecc. Maximizer - Initial Peri.: 276.8 [deg] & 6.4 & 3.2 & 11.7 \\
\hline 98 & 1 & SMA and Ecc. Maximizer - Initial Peri.: 248.0 [deg] & 5.3 & 3.2 & 9.8 \\
\hline 99 & 1 & SMA Maximizer - Initial Peri.: 233.5 [deg] & 4.5 & 3.2 & 8.7 \\
\hline 100 & 1 & SMA and Ecc. Maximizer - Initial Peri.: 317.2 [deg] & 6.0 & 3.1 & 11.0 \\
\hline 101 & 1 & SMA Maximizer - Initial Peri.: 229.8 [deg] & 4.0 & 2.9 & 8.2 \\
\hline 102 & 1 & SMA and Ecc. Maximizer - Initial Peri.: 288.8 [deg] & 5.5 & 2.9 & 10.5 \\
\hline 103 & 1 & SMA and Ecc. Maximizer - Initial Peri.: 268.7 [deg] & 4.9 & 2.8 & 9.8 \\
\hline 104 & 1 & SMA Maximizer w/Constant Ecc. & 3.5 & 2.8 & 7.6 \\
\hline 105 & 1 & SMA Maximizer - Initial Peri.: 179.9 [deg] & 3.0 & 2.8 & 6.7 \\
\hline 106 & 1 & SMA and Ecc. Maximizer - Initial Peri.: 19.7 [deg] & 3.3 & 2.7 & 7.3 \\
\hline 107 & 1 & SMA and Ecc. Maximizer - Initial Peri.: 218.2 [deg] & 3.3 & 2.6 & 7.4 \\
\hline 108 & 1 & SMA Maximizer w/Constant Ecc. & 3.2 & 2.5 & 7.1 \\
\hline 109 & 1 & SMA Maximizer - Initial Peri.: 26.5 [deg] & 2.9 & 2.5 & 7.1 \\
\hline
\end{tabular}




\begin{tabular}{|c|c|c|c|c|c|}
\hline Solution & Vehicle & Maneuver & $\begin{array}{c}\text { Propellant } \\
\text { Consumed by } \\
\text { Constellation } \\
{[\mathrm{kg}]}\end{array}$ & $\begin{array}{c}\text { Total Coverage } \\
\text { Time [hr] } \\
\text { Washington, } \\
\text { D.C. }\end{array}$ & $\begin{array}{c}\text { Total Coverage } \\
\text { Time [hr] } \\
\text { Montevideo }\end{array}$ \\
\hline 110 & 1 & SMA Maximizer - Initial Peri.: 189.8 [deg] & 2.8 & 2.5 & 6.7 \\
\hline 111 & 1 & SMA Maximizer - Initial Peri.: 272.6 [deg] & 3.7 & 2.3 & 8.4 \\
\hline 112 & 1 & SMA Maximizer - Initial Peri.: 313.2 [deg] & 3.7 & 2.3 & 8.5 \\
\hline 113 & 1 & SMA and Ecc. Maximizer - Initial Peri.: 147.8 [deg] & 2.2 & 2.2 & 6.1 \\
\hline 114 & 1 & SMA and Ecc. Maximizer - Initial Peri.: 143.0 [deg] & 2.1 & 2.2 & 6.1 \\
\hline 115 & 1 & SMA Maximizer - Initial Peri.: 213.5 [deg] & 2.5 & 2.1 & 6.7 \\
\hline 116 & 1 & SMA and Ecc. Maximizer - Initial Peri.: 96.4 [deg] & 2.0 & 2.1 & 6.0 \\
\hline 117 & 1 & SMA and Ecc. Maximizer - Initial Peri.: 19.7 [deg] & 2.3 & 2.0 & 6.6 \\
\hline 118 & 1 & SMA Maximizer - Initial Peri.: 325.7 [deg] & 3.1 & 2.0 & 7.6 \\
\hline 119 & 1 & SMA and Ecc. Maximizer - Initial Peri.: 93.4 [deg] & 1.8 & 1.9 & 5.8 \\
\hline 120 & 1 & SMA and Ecc. Maximizer - Initial Peri.: 305.0 [deg] & 2.8 & 1.8 & 7.5 \\
\hline 121 & 1 & SMA and Ecc. Maximizer - Initial Peri.: 248.9 [deg] & 2.3 & 1.8 & 6.9 \\
\hline 122 & 1 & SMA Maximizer - Initial Peri.: 158.2 [deg] & 1.7 & 1.7 & 5.9 \\
\hline 123 & 1 & SMA Maximizer - Initial Peri.: 229.8 [deg] & 1.9 & 1.6 & 6.3 \\
\hline 124 & 1 & SMA Maximizer - Initial Peri.: 254.7 [deg] & 2.0 & 1.5 & 6.5 \\
\hline 125 & 1 & SMA Maximizer - Initial Peri.: 307.9 [deg] & 2.1 & 1.5 & 6.7 \\
\hline 126 & 1 & SMA Maximizer - Initial Peri.: 116.8 [deg] & 1.3 & 1.5 & 5.6 \\
\hline 127 & 1 & SMA and Ecc. Maximizer - Initial Peri.: 63.5 [deg] & 1.4 & 1.4 & 5.7 \\
\hline 128 & 1 & SMA Maximizer w/Constant Ecc. & 1.4 & 1.3 & 5.9 \\
\hline 129 & 1 & SMA Maximizer - Initial Peri.: 359.2 [deg] & 1.5 & 1.3 & 6.0 \\
\hline 130 & 1 & SMA Maximizer - Initial Peri.: 195.9 [deg] & 1.2 & 1.3 & 5.7 \\
\hline 131 & 1 & SMA Maximizer - Initial Peri.: 167.6 [deg] & 1.1 & 1.2 & 5.5 \\
\hline
\end{tabular}




\begin{tabular}{|c|c|c|c|c|c|}
\hline Solution & Vehicle & Maneuver & $\begin{array}{c}\text { Propellant } \\
\text { Consumed by } \\
\text { Constellation } \\
\text { [kg] }\end{array}$ & $\begin{array}{c}\text { Total Coverage } \\
\text { Time [hr] } \\
\text { Washington, } \\
\text { D.C. }\end{array}$ & $\begin{array}{c}\text { Total Coverage } \\
\text { Time [hr] } \\
\text { Montevideo }\end{array}$ \\
\hline 132 & 1 & SMA Maximizer - Initial Peri.: 9.3 [deg] & 0.9 & 1.0 & 5.5 \\
\hline 133 & 1 & Ecc. Maximizer - Initial Peri.: 99.2 [deg] & 0.7 & 0.9 & 5.2 \\
\hline 134 & 1 & SMA Maximizer - Initial Peri.: 352.3 [deg] & 0.8 & 0.9 & 5.4 \\
\hline 135 & 1 & SMA Maximizer - Initial Peri.: 349.0 [deg] & 0.7 & 0.8 & 5.4 \\
\hline 136 & 1 & Ecc. Maximizer - Initial Peri.: 3.2 [deg] & 0.5 & 0.8 & 5.2 \\
\hline 137 & 1 & SMA Maximizer - Initial Peri.: 237.8 [deg] & 0.5 & 0.7 & 5.2 \\
\hline 138 & 1 & SMA Maximizer - Initial Peri.: 278.5 [deg] & 0.5 & 0.6 & 5.2 \\
\hline 139 & 1 & SMA and Ecc. Maximizer - Initial Peri.: 303.5 [deg] & 0.3 & 0.6 & 5.1 \\
\hline 140 & 1 & SMA and Ecc. Maximizer - Initial Peri.: 237.6 [deg] & 0.3 & 0.5 & 5.0 \\
\hline 141 & 1 & SMA Maximizer - Initial Peri.: 237.6 [deg] & 0.3 & 0.5 & 5.0 \\
\hline 142 & 1 & SMA and Ecc. Maximizer - Initial Peri.: 243.7 [deg] & 0.1 & 0.5 & 4.8 \\
\hline 143 & 1 & Zero maneuver & 0.0 & 0.4 & 4.7 \\
\hline 144 & 1 & Incl. Minimizer - Initial RAAN: 189.1 [deg] & 0.1 & 0.4 & 4.7 \\
\hline
\end{tabular}


Table 19: Final nondominated surface for Example 6.3

\begin{tabular}{|c|c|c|c|c|c|c|}
\hline Solution & Vehicle & Maneuver & $\begin{array}{c}\text { Propellant } \\
\text { Consumed by } \\
\text { Vehicle [kg] }\end{array}$ & $\begin{array}{c}\text { Propellant } \\
\text { Consumed by } \\
\text { Constellation } \\
{[\mathrm{kg}]}\end{array}$ & $\begin{array}{c}\text { Total } \\
\text { Coverage } \\
\text { Time [hr] } \\
\text { Washington, } \\
\text { D.C. }\end{array}$ & $\begin{array}{c}\text { Total } \\
\text { Coverage } \\
\text { Time [hr] } \\
\text { Montevideo }\end{array}$ \\
\hline 1 & 1 & SMA Maximizer - Initial Peri.: 171.7 [deg] & 9.1 & 28.6 & 249.8 & 93.2 \\
\hline 1 & 2 & SMA Maximizer - Initial Peri.: 266.1 [deg] & 9.9 & 28.6 & 249.8 & 93.2 \\
\hline 1 & 3 & SMA Maximizer & 9.7 & 28.6 & 249.8 & 93.2 \\
\hline 2 & 1 & SMA and Ecc. Maximizer - Initial Peri.: 120.3 [deg] & 9.7 & 29.3 & 248.2 & 100.2 \\
\hline 2 & 2 & SMA Maximizer - Initial Peri.: 84.0 [deg] & 9.9 & 29.3 & 248.2 & 100.2 \\
\hline 2 & 3 & SMA Maximizer & 9.7 & 29.3 & 248.2 & 100.2 \\
\hline 3 & 1 & SMA and Ecc. Maximizer - Initial Peri.: 120.3 [deg] & 9.7 & 29.4 & 246.6 & 102.8 \\
\hline 3 & 2 & SMA Maximizer - Initial Peri.: 45.9 [deg] & 10.0 & 29.4 & 246.6 & 102.8 \\
\hline 3 & 3 & SMA Maximizer & 9.7 & 29.4 & 246.6 & 102.8 \\
\hline 4 & 1 & SMA and Ecc. Maximizer - Initial Peri.: 43.0 [deg] & 10.0 & 29.6 & 246.6 & 103.8 \\
\hline 4 & 2 & SMA Maximizer - Initial Peri.: 31.2 [deg] & 10.0 & 29.6 & 246.6 & 103.8 \\
\hline 4 & 3 & SMA Maximizer & 9.7 & 29.6 & 246.6 & 103.8 \\
\hline 5 & 1 & SMA and Ecc. Maximizer - Initial Peri.: 28.2 [deg] & 9.1 & 27.5 & 242.2 & 95.8 \\
\hline 5 & 2 & SMA Maximizer - Initial Peri.: 76.3 [deg] & 8.7 & 27.5 & 242.2 & 95.8 \\
\hline 5 & 3 & SMA Maximizer & 9.7 & 27.5 & 242.2 & 95.8 \\
\hline 6 & 1 & SMA Maximizer - Initial Peri.: 78.0 [deg] & 9.4 & 26.7 & 238.5 & 94.2 \\
\hline 6 & 2 & SMA Maximizer - Initial Peri.: 26.6 [deg] & 7.7 & 26.7 & 238.5 & 94.2 \\
\hline 6 & 3 & SMA Maximizer & 9.7 & 26.7 & 238.5 & 94.2 \\
\hline 7 & 1 & SMA and Ecc. Maximizer - Initial Peri.: 44.0 [deg] & 6.4 & 24.8 & 238.2 & 85.1 \\
\hline 7 & 2 & SMA Maximizer - Initial Peri.: 277.0 [deg] & 8.7 & 24.8 & 238.2 & 85.1 \\
\hline
\end{tabular}




\begin{tabular}{|c|c|c|c|c|c|c|}
\hline Solution & Vehicle & Maneuver & $\begin{array}{l}\text { Propellant } \\
\text { Consumed by } \\
\text { Vehicle [kg] }\end{array}$ & $\begin{array}{c}\text { Propellant } \\
\text { Consumed by } \\
\text { Constellation } \\
{[\mathrm{kg}]}\end{array}$ & $\begin{array}{c}\text { Total } \\
\text { Coverage } \\
\text { Time [hr] } \\
\text { Washington, } \\
\text { D.C. }\end{array}$ & $\begin{array}{c}\text { Total } \\
\text { Coverage } \\
\text { Time [hr] } \\
\text { Montevideo }\end{array}$ \\
\hline 7 & 3 & SMA Maximizer & 9.7 & 24.8 & 238.2 & 85.1 \\
\hline 8 & 1 & SMA and Ecc. Maximizer - Initial Peri.: 350.3 [deg] & 6.5 & 26.1 & 237.0 & 94.8 \\
\hline 8 & 2 & SMA and Ecc. Maximizer - Initial Peri.: 351.0 [deg] & 9.9 & 26.1 & 237.0 & 94.8 \\
\hline 8 & 3 & SMA Maximizer & 9.7 & 26.1 & 237.0 & 94.8 \\
\hline 9 & 1 & SMA and Ecc. Maximizer - Initial Peri.: 73.3 [deg] & 6.7 & 25.2 & 236.8 & 87.7 \\
\hline 9 & 2 & SMA Maximizer - Initial Peri.: 304.2 [deg] & 8.8 & 25.2 & 236.8 & 87.7 \\
\hline 9 & 3 & SMA Maximizer & 9.7 & 25.2 & 236.8 & 87.7 \\
\hline 10 & 1 & SMA Maximizer w/Constant Ecc. & 5.6 & 24.6 & 235.7 & 86.5 \\
\hline 10 & 2 & SMA and Ecc. Maximizer - Initial Peri.: 324.5 [deg] & 9.3 & 24.6 & 235.7 & 86.5 \\
\hline 10 & 3 & SMA Maximizer & 9.7 & 24.6 & 235.7 & 86.5 \\
\hline 11 & 1 & SMA Maximizer - Initial Peri.: 120.3 [deg] & 4.6 & 24.1 & 234.1 & 89.0 \\
\hline 11 & 2 & SMA Maximizer - Initial Peri.: 10.2 [deg] & 9.9 & 24.1 & 234.1 & 89.0 \\
\hline 11 & 3 & SMA Maximizer & 9.7 & 24.1 & 234.1 & 89.0 \\
\hline 12 & 1 & SMA and Ecc. Maximizer - Initial Peri.: 13.0 [deg] & 5.3 & 24.4 & 232.7 & 89.7 \\
\hline 12 & 2 & SMA Maximizer - Initial Peri.: 357.8 [deg] & 9.4 & 24.4 & 232.7 & 89.7 \\
\hline 12 & 3 & SMA Maximizer & 9.7 & 24.4 & 232.7 & 89.7 \\
\hline 13 & 1 & SMA Maximizer - Initial Peri.: 231.5 [deg] & 3.1 & 22.1 & 232.1 & 78.1 \\
\hline 13 & 2 & SMA Maximizer - Initial Peri.: 297.7 [deg] & 9.4 & 22.1 & 232.1 & 78.1 \\
\hline 13 & 3 & SMA Maximizer & 9.7 & 22.1 & 232.1 & 78.1 \\
\hline 14 & 1 & SMA Maximizer - Initial Peri.: 282.2 [deg] & 2.8 & 21.7 & 231.7 & 76.5 \\
\hline 14 & 2 & SMA Maximizer - Initial Peri.: 277.0 [deg] & 9.3 & 21.7 & 231.7 & 76.5 \\
\hline
\end{tabular}




\begin{tabular}{|c|c|c|c|c|c|c|}
\hline Solution & Vehicle & Maneuver & $\begin{array}{c}\text { Propellant } \\
\text { Consumed by } \\
\text { Vehicle [kg] }\end{array}$ & $\begin{array}{c}\text { Propellant } \\
\text { Consumed by } \\
\text { Constellation } \\
{[\mathrm{kg}]}\end{array}$ & $\begin{array}{c}\text { Total } \\
\text { Coverage } \\
\text { Time [hr] } \\
\text { Washington, } \\
\text { D.C. }\end{array}$ & $\begin{array}{c}\text { Total } \\
\text { Coverage } \\
\text { Time [hr] } \\
\text { Montevideo }\end{array}$ \\
\hline 14 & 3 & SMA Maximizer & 9.7 & 21.7 & 231.7 & 76.5 \\
\hline 15 & 1 & SMA Maximizer - Initial Peri.: 231.5 [deg] & 3.1 & 21.3 & 230.2 & 75.7 \\
\hline 15 & 2 & SMA Maximizer - Initial Peri.: 297.7 [deg] & 8.6 & 21.3 & 230.2 & 75.7 \\
\hline 15 & 3 & SMA Maximizer & 9.7 & 21.3 & 230.2 & 75.7 \\
\hline 16 & 1 & Zero maneuver & 0.0 & 19.5 & 229.8 & 69.5 \\
\hline 16 & 2 & SMA Maximizer - Initial Peri.: 243.3 [deg] & 9.9 & 19.5 & 229.8 & 69.5 \\
\hline 16 & 3 & SMA Maximizer & 9.7 & 19.5 & 229.8 & 69.5 \\
\hline 17 & 1 & SMA and Ecc. Maximizer - Initial Peri.: 39.8 [deg] & 6.9 & 25.4 & 229.5 & 90.1 \\
\hline 17 & 2 & SMA Maximizer - Initial Peri.: 351.0 [deg] & 8.6 & 25.4 & 229.5 & 90.1 \\
\hline 17 & 3 & SMA Maximizer & 9.9 & 25.4 & 229.5 & 90.1 \\
\hline 18 & 1 & SMA Maximizer - Initial Peri.: 231.5 [deg] & 0.4 & 18.6 & 227.4 & 65.0 \\
\hline 18 & 2 & SMA Maximizer - Initial Peri.: 165.1 [deg] & 8.6 & 18.6 & 227.4 & 65.0 \\
\hline 18 & 3 & SMA Maximizer & 9.7 & 18.6 & 227.4 & 65.0 \\
\hline 19 & 1 & SMA Maximizer - Initial Peri.: 8.9 [deg] & 9.4 & 25.7 & 224.6 & 92.8 \\
\hline 19 & 2 & SMA Maximizer - Initial Peri.: 26.6 [deg] & 7.7 & 25.7 & 224.6 & 92.8 \\
\hline 19 & 3 & SMA Maximizer & 8.7 & 25.7 & 224.6 & 92.8 \\
\hline 20 & 1 & Zero maneuver & 0.0 & 19.0 & 223.3 & 75.1 \\
\hline 20 & 2 & SMA Maximizer - Initial Peri.: 51.6 [deg] & 9.3 & 19.0 & 223.3 & 75.1 \\
\hline 20 & 3 & SMA Maximizer & 9.7 & 19.0 & 223.3 & 75.1 \\
\hline 21 & 1 & SMA and Ecc. Maximizer - Initial Peri.: 349.9 [deg] & 6.5 & 24.7 & 222.7 & 94.2 \\
\hline 21 & 2 & SMA Maximizer - Initial Peri.: 45.9 [deg] & 9.9 & 24.7 & 222.7 & 94.2 \\
\hline
\end{tabular}




\begin{tabular}{|c|c|c|c|c|c|c|}
\hline Solution & Vehicle & Maneuver & $\begin{array}{l}\text { Propellant } \\
\text { Consumed by } \\
\text { Vehicle [kg] }\end{array}$ & $\begin{array}{c}\text { Propellant } \\
\text { Consumed by } \\
\text { Constellation } \\
{[\mathrm{kg}]}\end{array}$ & $\begin{array}{c}\text { Total } \\
\text { Coverage } \\
\text { Time [hr] } \\
\text { Washington, } \\
\text { D.C. }\end{array}$ & $\begin{array}{c}\text { Total } \\
\text { Coverage } \\
\text { Time [hr] } \\
\text { Montevideo }\end{array}$ \\
\hline 21 & 3 & SMA Maximizer & 8.3 & 24.7 & 222.7 & 94.2 \\
\hline 22 & 1 & SMA Maximizer w/Constant Ecc. & 5.0 & 23.3 & 222.5 & 86.0 \\
\hline 22 & 2 & SMA Maximizer - Initial Peri.: 69.2 [deg] & 9.5 & 23.3 & 222.5 & 86.0 \\
\hline 22 & 3 & SMA and Ecc. Maximizer & 8.8 & 23.3 & 222.5 & 86.0 \\
\hline 23 & 1 & SMA Maximizer - Initial Peri.: 340.1 [deg] & 7.6 & 24.1 & 220.2 & 88.8 \\
\hline 23 & 2 & SMA Maximizer - Initial Peri.: 75.6 [deg] & 8.4 & 24.1 & 220.2 & 88.8 \\
\hline 23 & 3 & SMA Maximizer & 8.2 & 24.1 & 220.2 & 88.8 \\
\hline 24 & 1 & SMA and Ecc. Maximizer - Initial Peri.: 350.3 [deg] & 1.3 & 18.3 & 218.9 & 71.4 \\
\hline 24 & 2 & SMA and Ecc. Maximizer - Initial Peri.: 351.0 [deg] & 7.3 & 18.3 & 218.9 & 71.4 \\
\hline 24 & 3 & SMA Maximizer & 9.7 & 18.3 & 218.9 & 71.4 \\
\hline 25 & 1 & Zero maneuver & 0.0 & 16.1 & 218.4 & 60.1 \\
\hline 25 & 2 & SMA Maximizer - Initial Peri.: 266.1 [deg] & 6.4 & 16.1 & 218.4 & 60.1 \\
\hline 25 & 3 & SMA Maximizer & 9.7 & 16.1 & 218.4 & 60.1 \\
\hline 26 & 1 & SMA Maximizer w/Constant Ecc. & 5.1 & 22.9 & 216.0 & 88.3 \\
\hline 26 & 2 & SMA Maximizer - Initial Peri.: 37.3 [deg] & 9.9 & 22.9 & 216.0 & 88.3 \\
\hline 26 & 3 & SMA Maximizer & 8.0 & 22.9 & 216.0 & 88.3 \\
\hline 27 & 1 & SMA Maximizer w/Constant Ecc. & 3.2 & 21.5 & 215.4 & 83.6 \\
\hline 27 & 2 & SMA Maximizer - Initial Peri.: 50.2 [deg] & 10.0 & 21.5 & 215.4 & 83.6 \\
\hline 27 & 3 & SMA Maximizer & 8.3 & 21.5 & 215.4 & 83.6 \\
\hline 28 & 1 & Zero maneuver & 0.0 & 15.8 & 213.9 & 64.1 \\
\hline 28 & 2 & SMA Maximizer - Initial Peri.: 6.7 [deg] & 6.2 & 15.8 & 213.9 & 64.1 \\
\hline
\end{tabular}




\begin{tabular}{|c|c|c|c|c|c|c|}
\hline Solution & Vehicle & Maneuver & $\begin{array}{l}\text { Propellant } \\
\text { Consumed by } \\
\text { Vehicle [kg] }\end{array}$ & $\begin{array}{c}\text { Propellant } \\
\text { Consumed by } \\
\text { Constellation } \\
{[\mathrm{kg}]}\end{array}$ & $\begin{array}{c}\text { Total } \\
\text { Coverage } \\
\text { Time [hr] } \\
\text { Washington, } \\
\text { D.C. }\end{array}$ & $\begin{array}{c}\text { Total } \\
\text { Coverage } \\
\text { Time [hr] } \\
\text { Montevideo }\end{array}$ \\
\hline 28 & 3 & SMA Maximizer & 9.7 & 15.8 & 213.9 & 64.1 \\
\hline 29 & 1 & Zero maneuver & 0.0 & 15.5 & 213.5 & 62.8 \\
\hline 29 & 2 & SMA and Ecc. Maximizer - Initial Peri.: 26.6 [deg] & 5.9 & 15.5 & 213.5 & 62.8 \\
\hline 29 & 3 & SMA Maximizer & 9.7 & 15.5 & 213.5 & 62.8 \\
\hline 30 & 1 & Zero maneuver & 0.0 & 15.2 & 213.2 & 61.2 \\
\hline 30 & 2 & SMA Maximizer - Initial Peri.: 45.9 [deg] & 5.6 & 15.2 & 213.2 & 61.2 \\
\hline 30 & 3 & SMA Maximizer & 9.7 & 15.2 & 213.2 & 61.2 \\
\hline 31 & 1 & SMA Maximizer w/Constant Ecc. & 3.2 & 19.9 & 210.7 & 77.8 \\
\hline 31 & 2 & SMA Maximizer - Initial Peri.: 50.2 [deg] & 8.4 & 19.9 & 210.7 & 77.8 \\
\hline 31 & 3 & SMA Maximizer & 8.3 & 19.9 & 210.7 & 77.8 \\
\hline 32 & 1 & SMA and Ecc. Maximizer - Initial Peri.: 277.4 [deg] & 0.1 & 17.9 & 209.3 & 73.9 \\
\hline 32 & 2 & SMA and Ecc. Maximizer - Initial Peri.: 352.8 [deg] & 9.2 & 17.9 & 209.3 & 73.9 \\
\hline 32 & 3 & SMA Maximizer & 8.7 & 17.9 & 209.3 & 73.9 \\
\hline 33 & 1 & Zero maneuver & 0.0 & 17.5 & 207.6 & 73.1 \\
\hline 33 & 2 & SMA Maximizer - Initial Peri.: 26.6 [deg] & 8.9 & 17.5 & 207.6 & 73.1 \\
\hline 33 & 3 & SMA Maximizer & 8.6 & 17.5 & 207.6 & 73.1 \\
\hline 34 & 1 & SMA Maximizer - Initial Peri.: 9.3 [deg] & 9.9 & 29.7 & 205.4 & 114.5 \\
\hline 34 & 2 & SMA Maximizer - Initial Peri.: 89.1 [deg] & 9.9 & 29.7 & 205.4 & 114.5 \\
\hline 34 & 3 & SMA Maximizer w/Constant Ecc. & 10.0 & 29.7 & 205.4 & 114.5 \\
\hline 35 & 1 & SMA Maximizer - Initial Peri.: 187.6 [deg] & 9.6 & 27.8 & 203.9 & 102.4 \\
\hline 35 & 2 & SMA and Ecc. Maximizer - Initial Peri.: 148.8 [deg] & 8.6 & 27.8 & 203.9 & 102.4 \\
\hline
\end{tabular}




\begin{tabular}{|c|c|c|c|c|c|c|}
\hline Solution & Vehicle & Maneuver & $\begin{array}{l}\text { Propellant } \\
\text { Consumed by } \\
\text { Vehicle [kg] }\end{array}$ & $\begin{array}{c}\text { Propellant } \\
\text { Consumed by } \\
\text { Constellation } \\
{[\mathrm{kg}]}\end{array}$ & $\begin{array}{c}\text { Total } \\
\text { Coverage } \\
\text { Time [hr] } \\
\text { Washington, } \\
\text { D.C. }\end{array}$ & $\begin{array}{c}\text { Total } \\
\text { Coverage } \\
\text { Time [hr] } \\
\text { Montevideo }\end{array}$ \\
\hline 35 & 3 & SMA Maximizer w/Constant Ecc. & 9.6 & 27.8 & 203.9 & 102.4 \\
\hline 36 & 1 & SMA and Ecc. Maximizer - Initial Peri.: 62.8 [deg] & 9.9 & 29.8 & 203.8 & 117.2 \\
\hline 36 & 2 & SMA Maximizer - Initial Peri.: 26.6 [deg] & 9.9 & 29.8 & 203.8 & 117.2 \\
\hline 36 & 3 & SMA Maximizer w/Constant Ecc. & 10.0 & 29.8 & 203.8 & 117.2 \\
\hline 37 & 1 & SMA and Ecc. Maximizer - Initial Peri.: 137.8 [deg] & 10.0 & 28.4 & 203.6 & 106.8 \\
\hline 37 & 2 & SMA Maximizer - Initial Peri.: 277.0 [deg] & 8.7 & 28.4 & 203.6 & 106.8 \\
\hline 37 & 3 & SMA Maximizer w/Constant Ecc. & 9.6 & 28.4 & 203.6 & 106.8 \\
\hline 38 & 1 & SMA and Ecc. Maximizer - Initial Peri.: 19.2 [deg] & 9.8 & 29.6 & 203.1 & 117.0 \\
\hline 38 & 2 & SMA Maximizer - Initial Peri.: 26.6 [deg] & 9.9 & 29.6 & 203.1 & 117.0 \\
\hline 38 & 3 & SMA Maximizer w/Constant Ecc. & 10.0 & 29.6 & 203.1 & 117.0 \\
\hline 39 & 1 & SMA Maximizer - Initial Peri.: 120.3 [deg] & 9.7 & 29.4 & 202.5 & 115.6 \\
\hline 39 & 2 & SMA Maximizer - Initial Peri.: 45.9 [deg] & 10.0 & 29.4 & 202.5 & 115.6 \\
\hline 39 & 3 & SMA Maximizer w/Constant Ecc. & 9.7 & 29.4 & 202.5 & 115.6 \\
\hline 40 & 1 & SMA and Ecc. Maximizer - Initial Peri.: 61.4 [deg] & 9.6 & 27.9 & 202.3 & 105.8 \\
\hline 40 & 2 & SMA Maximizer - Initial Peri.: 277.0 [deg] & 8.7 & 27.9 & 202.3 & 105.8 \\
\hline 40 & 3 & SMA Maximizer w/Constant Ecc. & 9.6 & 27.9 & 202.3 & 105.8 \\
\hline 41 & 1 & SMA Maximizer - Initial Peri.: 178.4 [deg] & 10.0 & 28.9 & 201.9 & 113.4 \\
\hline 41 & 2 & SMA Maximizer - Initial Peri.: 342.3 [deg] & 8.9 & 28.9 & 201.9 & 113.4 \\
\hline 41 & 3 & SMA Maximizer w/Constant Ecc. & 10.0 & 28.9 & 201.9 & 113.4 \\
\hline 42 & 1 & SMA and Ecc. Maximizer - Initial Peri.: 31.1 [deg] & 9.1 & 27.5 & 200.1 & 105.3 \\
\hline 42 & 2 & SMA Maximizer - Initial Peri.: 277.0 [deg] & 8.7 & 27.5 & 200.1 & 105.3 \\
\hline
\end{tabular}




\begin{tabular}{|c|c|c|c|c|c|c|}
\hline Solution & Vehicle & Maneuver & $\begin{array}{c}\text { Propellant } \\
\text { Consumed by } \\
\text { Vehicle [kg] }\end{array}$ & $\begin{array}{c}\text { Propellant } \\
\text { Consumed by } \\
\text { Constellation } \\
{[\mathrm{kg}]}\end{array}$ & $\begin{array}{c}\text { Total } \\
\text { Coverage } \\
\text { Time [hr] } \\
\text { Washington, } \\
\text { D.C. }\end{array}$ & $\begin{array}{c}\text { Total } \\
\text { Coverage } \\
\text { Time [hr] } \\
\text { Montevideo }\end{array}$ \\
\hline 42 & 3 & SMA Maximizer w/Constant Ecc. & 9.6 & 27.5 & 200.1 & 105.3 \\
\hline 43 & 1 & Zero maneuver & 0.0 & 11.6 & 199.6 & 43.3 \\
\hline 43 & 2 & SMA Maximizer w/Constant Ecc. & 1.7 & 11.6 & 199.6 & 43.3 \\
\hline 43 & 3 & SMA Maximizer & 9.9 & 11.6 & 199.6 & 43.3 \\
\hline 44 & 1 & SMA Maximizer - Initial Peri.: 340.1 [deg] & 7.6 & 27.4 & 198.6 & 110.6 \\
\hline 44 & 2 & SMA Maximizer - Initial Peri.: 75.6 [deg] & 10.0 & 27.4 & 198.6 & 110.6 \\
\hline 44 & 3 & SMA Maximizer w/Constant Ecc. & 9.9 & 27.4 & 198.6 & 110.6 \\
\hline 45 & 1 & SMA Maximizer w/Constant Ecc. & 3.2 & 14.7 & 198.5 & 55.0 \\
\hline 45 & 2 & SMA Maximizer - Initial Peri.: 266.1 [deg] & 3.2 & 14.7 & 198.5 & 55.0 \\
\hline 45 & 3 & SMA Maximizer & 8.3 & 14.7 & 198.5 & 55.0 \\
\hline 46 & 1 & SMA and Ecc. Maximizer - Initial Peri.: 13.0 [deg] & 9.1 & 28.0 & 198.2 & 111.7 \\
\hline 46 & 2 & SMA Maximizer - Initial Peri.: 50.5 [deg] & 9.2 & 28.0 & 198.2 & 111.7 \\
\hline 46 & 3 & SMA Maximizer w/Constant Ecc. & 9.7 & 28.0 & 198.2 & 111.7 \\
\hline 47 & 1 & SMA Maximizer w/Constant Ecc. & 9.1 & 22.4 & 196.0 & 91.1 \\
\hline 47 & 2 & SMA Maximizer - Initial Peri.: 92.1 [deg] & 8.9 & 22.4 & 196.0 & 91.1 \\
\hline 47 & 3 & SMA Maximizer & 4.4 & 22.4 & 196.0 & 91.1 \\
\hline 48 & 1 & SMA and Ecc. Maximizer - Initial Peri.: 37.1 [deg] & 9.5 & 27.3 & 195.6 & 108.5 \\
\hline 48 & 2 & SMA and Ecc. Maximizer - Initial Peri.: 89.7 [deg] & 9.9 & 27.3 & 195.6 & 108.5 \\
\hline 48 & 3 & SMA Maximizer w/Constant Ecc. & 7.8 & 27.3 & 195.6 & 108.5 \\
\hline 49 & 1 & SMA and Ecc. Maximizer - Initial Peri.: 61.4 [deg] & 9.1 & 25.7 & 195.2 & 99.1 \\
\hline 49 & 2 & SMA Maximizer w/Constant Ecc. & 6.8 & 25.7 & 195.2 & 99.1 \\
\hline
\end{tabular}




\begin{tabular}{|c|c|c|c|c|c|c|}
\hline Solution & Vehicle & Maneuver & $\begin{array}{l}\text { Propellant } \\
\text { Consumed by } \\
\text { Vehicle [kg] }\end{array}$ & $\begin{array}{c}\text { Propellant } \\
\text { Consumed by } \\
\text { Constellation } \\
{[\mathrm{kg}]}\end{array}$ & $\begin{array}{c}\text { Total } \\
\text { Coverage } \\
\text { Time [hr] } \\
\text { Washington, } \\
\text { D.C. }\end{array}$ & $\begin{array}{c}\text { Total } \\
\text { Coverage } \\
\text { Time [hr] } \\
\text { Montevideo }\end{array}$ \\
\hline 49 & 3 & SMA Maximizer w/Constant Ecc. & 9.8 & 25.7 & 195.2 & 99.1 \\
\hline 50 & 1 & SMA and Ecc. Maximizer - Initial Peri.: 350.3 [deg] & 6.5 & 26.5 & 195.2 & 109.1 \\
\hline 50 & 2 & SMA and Ecc. Maximizer - Initial Peri.: 351.0 [deg] & 10.0 & 26.5 & 195.2 & 109.1 \\
\hline 50 & 3 & SMA Maximizer w/Constant Ecc. & 10.0 & 26.5 & 195.2 & 109.1 \\
\hline 51 & 1 & SMA Maximizer - Initial Peri.: 340.1 [deg] & 7.6 & 25.9 & 194.2 & 104.2 \\
\hline 51 & 2 & SMA Maximizer - Initial Peri.: 75.6 [deg] & 8.4 & 25.9 & 194.2 & 104.2 \\
\hline 51 & 3 & SMA Maximizer w/Constant Ecc. & 10.0 & 25.9 & 194.2 & 104.2 \\
\hline 52 & 1 & SMA Maximizer - Initial Peri.: 148.8 [deg] & 0.0 & 11.9 & 193.6 & 49.6 \\
\hline 52 & 2 & SMA Maximizer - Initial Peri.: 320.2 [deg] & 3.2 & 11.9 & 193.6 & 49.6 \\
\hline 52 & 3 & SMA Maximizer & 8.7 & 11.9 & 193.6 & 49.6 \\
\hline 53 & 1 & SMA and Ecc. Maximizer - Initial Peri.: 307.5 [deg] & 9.5 & 26.7 & 192.6 & 109.5 \\
\hline 53 & 2 & SMA Maximizer - Initial Peri.: 357.8 [deg] & 9.4 & 26.7 & 192.6 & 109.5 \\
\hline 53 & 3 & SMA Maximizer w/Constant Ecc. & 7.8 & 26.7 & 192.6 & 109.5 \\
\hline 54 & 1 & SMA Maximizer - Initial Peri.: 184.8 [deg] & 7.8 & 25.6 & 191.3 & 105.6 \\
\hline 54 & 2 & SMA Maximizer - Initial Peri.: 6.3 [deg] & 9.5 & 25.6 & 191.3 & 105.6 \\
\hline 54 & 3 & SMA Maximizer w/Constant Ecc. & 8.3 & 25.6 & 191.3 & 105.6 \\
\hline 55 & 1 & SMA and Ecc. Maximizer - Initial Peri.: 39.8 [deg] & 9.4 & 26.3 & 190.9 & 106.9 \\
\hline 55 & 2 & SMA Maximizer - Initial Peri.: 351.0 [deg] & 8.6 & 26.3 & 190.9 & 106.9 \\
\hline 55 & 3 & SMA Maximizer w/Constant Ecc. & 8.3 & 26.3 & 190.9 & 106.9 \\
\hline 56 & 1 & SMA and Ecc. Maximizer - Initial Peri.: 39.8 [deg] & 6.9 & 25.4 & 190.6 & 105.2 \\
\hline 56 & 2 & SMA Maximizer - Initial Peri.: 351.0 [deg] & 8.6 & 25.4 & 190.6 & 105.2 \\
\hline
\end{tabular}




\begin{tabular}{|c|c|c|c|c|c|c|}
\hline Solution & Vehicle & Maneuver & $\begin{array}{l}\text { Propellant } \\
\text { Consumed by } \\
\text { Vehicle [kg] }\end{array}$ & $\begin{array}{c}\text { Propellant } \\
\text { Consumed by } \\
\text { Constellation } \\
{[\mathrm{kg}]}\end{array}$ & $\begin{array}{c}\text { Total } \\
\text { Coverage } \\
\text { Time [hr] } \\
\text { Washington, } \\
\text { D.C. }\end{array}$ & $\begin{array}{c}\text { Total } \\
\text { Coverage } \\
\text { Time [hr] } \\
\text { Montevideo }\end{array}$ \\
\hline 56 & 3 & SMA Maximizer w/Constant Ecc. & 9.9 & 25.4 & 190.6 & 105.2 \\
\hline 57 & 1 & SMA and Ecc. Maximizer - Initial Peri.: 287.3 [deg] & 9.4 & 23.0 & 189.6 & 97.2 \\
\hline 57 & 2 & SMA Maximizer - Initial Peri.: 351.0 [deg] & 9.9 & 23.0 & 189.6 & 97.2 \\
\hline 57 & 3 & SMA Maximizer & 3.8 & 23.0 & 189.6 & 97.2 \\
\hline 58 & 1 & SMA Maximizer - Initial Peri.: 231.5 [deg] & 3.1 & 15.1 & 189.5 & 64.2 \\
\hline 58 & 2 & SMA Maximizer - Initial Peri.: 297.7 [deg] & 5.8 & 15.1 & 189.5 & 64.2 \\
\hline 58 & 3 & SMA Maximizer & 6.3 & 15.1 & 189.5 & 64.2 \\
\hline 59 & 1 & Incl. Maximizer - Initial RAAN: 225.2 [deg] & 1.6 & 15.9 & 187.9 & 68.2 \\
\hline 59 & 2 & SMA Maximizer - Initial Peri.: 100.4 [deg] & 8.6 & 15.9 & 187.9 & 68.2 \\
\hline 59 & 3 & SMA and Ecc. Maximizer & 5.7 & 15.9 & 187.9 & 68.2 \\
\hline 60 & 1 & Zero maneuver & 0.0 & 20.0 & 187.2 & 84.1 \\
\hline 60 & 2 & SMA Maximizer - Initial Peri.: 243.3 [deg] & 10.0 & 20.0 & 187.2 & 84.1 \\
\hline 60 & 3 & SMA Maximizer w/Constant Ecc. & 10.0 & 20.0 & 187.2 & 84.1 \\
\hline 61 & 1 & SMA and Ecc. Maximizer - Initial Peri.: 13.0 [deg] & 4.7 & 23.7 & 187.2 & 101.5 \\
\hline 61 & 2 & SMA Maximizer - Initial Peri.: 357.8 [deg] & 9.4 & 23.7 & 187.2 & 101.5 \\
\hline 61 & 3 & SMA Maximizer w/Constant Ecc. & 9.7 & 23.7 & 187.2 & 101.5 \\
\hline 62 & 1 & SMA and Ecc. Maximizer - Initial Peri.: 31.1 [deg] & 1.8 & 21.0 & 186.7 & 88.4 \\
\hline 62 & 2 & SMA and Ecc. Maximizer - Initial Peri.: 277.0 [deg] & 9.6 & 21.0 & 186.7 & 88.4 \\
\hline 62 & 3 & SMA Maximizer w/Constant Ecc. & 9.6 & 21.0 & 186.7 & 88.4 \\
\hline 63 & 1 & SMA Maximizer - Initial Peri.: 231.5 [deg] & 9.7 & 22.0 & 185.2 & 97.3 \\
\hline 63 & 2 & SMA Maximizer - Initial Peri.: 26.6 [deg] & 9.9 & 22.0 & 185.2 & 97.3 \\
\hline
\end{tabular}




\begin{tabular}{|c|c|c|c|c|c|c|}
\hline Solution & Vehicle & Maneuver & $\begin{array}{l}\text { Propellant } \\
\text { Consumed by } \\
\text { Vehicle [kg] }\end{array}$ & $\begin{array}{c}\text { Propellant } \\
\text { Consumed by } \\
\text { Constellation } \\
{[\mathrm{kg}]}\end{array}$ & $\begin{array}{c}\text { Total } \\
\text { Coverage } \\
\text { Time [hr] } \\
\text { Washington, } \\
\text { D.C. }\end{array}$ & $\begin{array}{c}\text { Total } \\
\text { Coverage } \\
\text { Time [hr] } \\
\text { Montevideo }\end{array}$ \\
\hline 63 & 3 & SMA Maximizer & 2.3 & 22.0 & 185.2 & 97.3 \\
\hline 64 & 1 & SMA and Ecc. Maximizer - Initial Peri.: 8.9 [deg] & 0.0 & 9.9 & 185.1 & 39.0 \\
\hline 64 & 2 & RAAN Minimizer & 1.2 & 9.9 & 185.1 & 39.0 \\
\hline 64 & 3 & SMA Maximizer & 8.7 & 9.9 & 185.1 & 39.0 \\
\hline 65 & 1 & SMA Maximizer - Initial Peri.: 105.8 [deg] & 1.1 & 21.0 & 184.8 & 92.5 \\
\hline 65 & 2 & SMA Maximizer - Initial Peri.: 69.2 [deg] & 9.9 & 21.0 & 184.8 & 92.5 \\
\hline 65 & 3 & SMA Maximizer w/Constant Ecc. & 10.0 & 21.0 & 184.8 & 92.5 \\
\hline 66 & 1 & SMA Maximizer - Initial Peri.: 37.1 [deg] & 0.3 & 20.2 & 184.3 & 89.8 \\
\hline 66 & 2 & SMA and Ecc. Maximizer - Initial Peri.: 89.7 [deg] & 9.9 & 20.2 & 184.3 & 89.8 \\
\hline 66 & 3 & SMA Maximizer w/Constant Ecc. & 10.0 & 20.2 & 184.3 & 89.8 \\
\hline 67 & 1 & SMA and Ecc. Maximizer - Initial Peri.: 13.0 [deg] & 2.7 & 21.3 & 182.2 & 94.4 \\
\hline 67 & 2 & SMA Maximizer - Initial Peri.: 6.3 [deg] & 9.0 & 21.3 & 182.2 & 94.4 \\
\hline 67 & 3 & SMA Maximizer w/Constant Ecc. & 9.7 & 21.3 & 182.2 & 94.4 \\
\hline 68 & 1 & SMA Maximizer - Initial Peri.: 307.5 [deg] & 0.4 & 19.7 & 180.5 & 90.6 \\
\hline 68 & 2 & SMA Maximizer - Initial Peri.: 357.8 [deg] & 9.4 & 19.7 & 180.5 & 90.6 \\
\hline 68 & 3 & SMA Maximizer w/Constant Ecc. & 10.0 & 19.7 & 180.5 & 90.6 \\
\hline 69 & 1 & Zero maneuver & 0.0 & 9.2 & 179.5 & 35.4 \\
\hline 69 & 2 & SMA and Ecc. Minimizer & 0.2 & 9.2 & 179.5 & 35.4 \\
\hline 69 & 3 & Ecc. Maximizer & 9.0 & 9.2 & 179.5 & 35.4 \\
\hline 70 & 1 & SMA Maximizer - Initial Peri.: 171.7 [deg] & 4.7 & 17.6 & 178.5 & 82.7 \\
\hline 70 & 2 & SMA Maximizer - Initial Peri.: 357.8 [deg] & 9.4 & 17.6 & 178.5 & 82.7 \\
\hline
\end{tabular}




\begin{tabular}{|c|c|c|c|c|c|c|}
\hline Solution & Vehicle & Maneuver & $\begin{array}{l}\text { Propellant } \\
\text { Consumed by } \\
\text { Vehicle [kg] }\end{array}$ & $\begin{array}{c}\text { Propellant } \\
\text { Consumed by } \\
\text { Constellation } \\
{[\mathrm{kg}]}\end{array}$ & $\begin{array}{c}\text { Total } \\
\text { Coverage } \\
\text { Time [hr] } \\
\text { Washington, } \\
\text { D.C. }\end{array}$ & $\begin{array}{c}\text { Total } \\
\text { Coverage } \\
\text { Time [hr] } \\
\text { Montevideo }\end{array}$ \\
\hline 70 & 3 & SMA Maximizer & 3.5 & 17.6 & 178.5 & 82.7 \\
\hline 71 & 1 & SMA and Ecc. Maximizer - Initial Peri.: 105.8 [deg] & 0.0 & 18.2 & 177.8 & 84.3 \\
\hline 71 & 2 & SMA Maximizer w/Constant Ecc. & 9.9 & 18.2 & 177.8 & 84.3 \\
\hline 71 & 3 & SMA Maximizer w/Constant Ecc. & 8.3 & 18.2 & 177.8 & 84.3 \\
\hline 72 & 1 & SMA and Ecc. Maximizer - Initial Peri.: 13.0 [deg] & 0.3 & 17.3 & 176.1 & 79.5 \\
\hline 72 & 2 & SMA Maximizer - Initial Peri.: 80.7 [deg] & 7.3 & 17.3 & 176.1 & 79.5 \\
\hline 72 & 3 & SMA Maximizer w/Constant Ecc. & 9.7 & 17.3 & 176.1 & 79.5 \\
\hline 73 & 1 & SMA and Ecc. Maximizer - Initial Peri.: 8.9 [deg] & 0.0 & 9.7 & 175.9 & 35.9 \\
\hline 73 & 2 & RAAN Minimizer & 1.2 & 9.7 & 175.9 & 35.9 \\
\hline 73 & 3 & Ecc. Maximizer & 8.4 & 9.7 & 175.9 & 35.9 \\
\hline 74 & 1 & SMA Maximizer - Initial Peri.: 120.3 [deg] & 4.6 & 17.3 & 173.0 & 85.3 \\
\hline 74 & 2 & SMA Maximizer - Initial Peri.: 13.6 [deg] & 9.9 & 17.3 & 173.0 & 85.3 \\
\hline 74 & 3 & SMA Maximizer & 2.9 & 17.3 & 173.0 & 85.3 \\
\hline 75 & 1 & SMA Maximizer - Initial Peri.: 350.3 [deg] & 0.3 & 13.4 & 171.6 & 68.1 \\
\hline 75 & 2 & SMA Maximizer - Initial Peri.: 146.1 [deg] & 10.0 & 13.4 & 171.6 & 68.1 \\
\hline 75 & 3 & SMA Maximizer & 3.1 & 13.4 & 171.6 & 68.1 \\
\hline 76 & 1 & Zero maneuver & 0.0 & 16.9 & 170.6 & 83.3 \\
\hline 76 & 2 & SMA Maximizer - Initial Peri.: 358.8 [deg] & 8.6 & 16.9 & 170.6 & 83.3 \\
\hline 76 & 3 & SMA Maximizer w/Constant Ecc. & 8.3 & 16.9 & 170.6 & 83.3 \\
\hline 77 & 1 & Zero maneuver & 0.0 & 16.6 & 169.2 & 82.9 \\
\hline 77 & 2 & SMA Maximizer - Initial Peri.: 358.8 [deg] & 8.6 & 16.6 & 169.2 & 82.9 \\
\hline
\end{tabular}




\begin{tabular}{|c|c|c|c|c|c|c|}
\hline Solution & Vehicle & Maneuver & $\begin{array}{l}\text { Propellant } \\
\text { Consumed by } \\
\text { Vehicle [kg] }\end{array}$ & $\begin{array}{c}\text { Propellant } \\
\text { Consumed by } \\
\text { Constellation } \\
{[\mathrm{kg}]}\end{array}$ & $\begin{array}{c}\text { Total } \\
\text { Coverage } \\
\text { Time [hr] } \\
\text { Washington, } \\
\text { D.C. }\end{array}$ & $\begin{array}{c}\text { Total } \\
\text { Coverage } \\
\text { Time [hr] } \\
\text { Montevideo }\end{array}$ \\
\hline 77 & 3 & SMA Maximizer w/Constant Ecc. & 8.0 & 16.6 & 169.2 & 82.9 \\
\hline 78 & 1 & SMA Maximizer w/Constant Ecc. & 9.6 & 20.7 & 168.3 & 95.7 \\
\hline 78 & 2 & SMA Maximizer - Initial Peri.: 81.3 [deg] & 9.9 & 20.7 & 168.3 & 95.7 \\
\hline 78 & 3 & SMA Maximizer & 1.2 & 20.7 & 168.3 & 95.7 \\
\hline 79 & 1 & Zero maneuver & 0.0 & 13.1 & 166.5 & 61.1 \\
\hline 79 & 2 & SMA Maximizer - Initial Peri.: 143.4 [deg] & 3.1 & 13.1 & 166.5 & 61.1 \\
\hline 79 & 3 & SMA Maximizer w/Constant Ecc. & 10.0 & 13.1 & 166.5 & 61.1 \\
\hline 80 & 1 & SMA Maximizer w/Constant Ecc. & 9.1 & 19.1 & 163.9 & 90.3 \\
\hline 80 & 2 & SMA Maximizer - Initial Peri.: 81.3 [deg] & 8.7 & 19.1 & 163.9 & 90.3 \\
\hline 80 & 3 & SMA Maximizer & 1.2 & 19.1 & 163.9 & 90.3 \\
\hline 81 & 1 & SMA Maximizer - Initial Peri.: 171.7 [deg] & 0.3 & 11.5 & 162.8 & 67.5 \\
\hline 81 & 2 & SMA Maximizer - Initial Peri.: 13.6 [deg] & 7.7 & 11.5 & 162.8 & 67.5 \\
\hline 81 & 3 & SMA Maximizer & 3.5 & 11.5 & 162.8 & 67.5 \\
\hline 82 & 1 & SMA Maximizer - Initial Peri.: 171.7 [deg] & 9.1 & 19.0 & 162.2 & 89.0 \\
\hline 82 & 2 & SMA Maximizer - Initial Peri.: 266.1 [deg] & 9.9 & 19.0 & 162.2 & 89.0 \\
\hline 82 & 3 & Zero maneuver & 0.0 & 19.0 & 162.2 & 89.0 \\
\hline 83 & 1 & Zero maneuver & 0.0 & 12.1 & 161.3 & 69.2 \\
\hline 83 & 2 & SMA Maximizer - Initial Peri.: 100.4 [deg] & 9.5 & 12.1 & 161.3 & 69.2 \\
\hline 83 & 3 & SMA Maximizer & 2.6 & 12.1 & 161.3 & 69.2 \\
\hline 84 & 1 & SMA Maximizer - Initial Peri.: 231.5 [deg] & 7.6 & 17.9 & 159.6 & 86.7 \\
\hline 84 & 2 & SMA Maximizer - Initial Peri.: 75.6 [deg] & 8.4 & 17.9 & 159.6 & 86.7 \\
\hline
\end{tabular}




\begin{tabular}{|c|c|c|c|c|c|c|}
\hline Solution & Vehicle & Maneuver & $\begin{array}{l}\text { Propellant } \\
\text { Consumed by } \\
\text { Vehicle [kg] }\end{array}$ & $\begin{array}{c}\text { Propellant } \\
\text { Consumed by } \\
\text { Constellation } \\
{[\mathrm{kg}]}\end{array}$ & $\begin{array}{c}\text { Total } \\
\text { Coverage } \\
\text { Time [hr] } \\
\text { Washington, } \\
\text { D.C. }\end{array}$ & $\begin{array}{c}\text { Total } \\
\text { Coverage } \\
\text { Time [hr] } \\
\text { Montevideo }\end{array}$ \\
\hline 84 & 3 & SMA Maximizer w/Constant Ecc. & 2.0 & 17.9 & 159.6 & 86.7 \\
\hline 85 & 1 & SMA Maximizer w/Constant Ecc. & 3.8 & 8.6 & 159.5 & 47.7 \\
\hline 85 & 2 & SMA Maximizer - Initial Peri.: 35.1 [deg] & 0.5 & 8.6 & 159.5 & 47.7 \\
\hline 85 & 3 & SMA Maximizer & 4.3 & 8.6 & 159.5 & 47.7 \\
\hline 86 & 1 & Zero maneuver & 0.0 & 10.9 & 159.1 & 56.2 \\
\hline 86 & 2 & SMA and Ecc. Maximizer - Initial Peri.: 105.5 [deg] & 0.9 & 10.9 & 159.1 & 56.2 \\
\hline 86 & 3 & SMA Maximizer w/Constant Ecc. & 10.0 & 10.9 & 159.1 & 56.2 \\
\hline 87 & 1 & SMA Maximizer - Initial Peri.: 340.1 [deg] & 9.9 & 20.3 & 158.8 & 97.8 \\
\hline 87 & 2 & SMA and Ecc. Maximizer - Initial Peri.: 70.6 [deg] & 9.9 & 20.3 & 158.8 & 97.8 \\
\hline 87 & 3 & Arg.Peri. Maximizer In-Plane Only & 0.5 & 20.3 & 158.8 & 97.8 \\
\hline 88 & 1 & SMA and Ecc. Maximizer - Initial Peri.: 43.0 [deg] & 10.0 & 20.0 & 158.0 & 99.7 \\
\hline 88 & 2 & SMA and Ecc. Maximizer - Initial Peri.: 31.2 [deg] & 10.0 & 20.0 & 158.0 & 99.7 \\
\hline 88 & 3 & Zero maneuver & 0.0 & 20.0 & 158.0 & 99.7 \\
\hline 89 & 1 & SMA Maximizer w/Constant Ecc. & 9.7 & 19.7 & 157.9 & 96.1 \\
\hline 89 & 2 & SMA and Ecc. Maximizer - Initial Peri.: 69.2 [deg] & 10.0 & 19.7 & 157.9 & 96.1 \\
\hline 89 & 3 & Zero maneuver & 0.0 & 19.7 & 157.9 & 96.1 \\
\hline 90 & 1 & SMA Maximizer - Initial Peri.: 231.5 [deg] & 0.0 & 10.9 & 157.4 & 67.5 \\
\hline 90 & 2 & SMA Maximizer - Initial Peri.: 43.5 [deg] & 8.1 & 10.9 & 157.4 & 67.5 \\
\hline 90 & 3 & SMA Maximizer & 2.8 & 10.9 & 157.4 & 67.5 \\
\hline 91 & 1 & SMA Maximizer w/Constant Ecc. & 2.7 & 14.8 & 154.9 & 78.7 \\
\hline 91 & 2 & SMA Maximizer - Initial Peri.: 76.3 [deg] & 8.6 & 14.8 & 154.9 & 78.7 \\
\hline
\end{tabular}




\begin{tabular}{|c|c|c|c|c|c|c|}
\hline Solution & Vehicle & Maneuver & $\begin{array}{c}\text { Propellant } \\
\text { Consumed by } \\
\text { Vehicle [kg] }\end{array}$ & $\begin{array}{c}\text { Propellant } \\
\text { Consumed by } \\
\text { Constellation } \\
{[\mathrm{kg}]}\end{array}$ & $\begin{array}{c}\text { Total } \\
\text { Coverage } \\
\text { Time [hr] } \\
\text { Washington, } \\
\text { D.C. }\end{array}$ & $\begin{array}{c}\text { Total } \\
\text { Coverage } \\
\text { Time [hr] } \\
\text { Montevideo }\end{array}$ \\
\hline 91 & 3 & SMA Maximizer w/Constant Ecc. & 3.5 & 14.8 & 154.9 & 78.7 \\
\hline 92 & 1 & Zero maneuver & 0.0 & 10.2 & 153.4 & 66.8 \\
\hline 92 & 2 & SMA Maximizer - Initial Peri.: 358.8 [deg] & 7.8 & 10.2 & 153.4 & 66.8 \\
\hline 92 & 3 & SMA Maximizer & 2.4 & 10.2 & 153.4 & 66.8 \\
\hline 93 & 1 & Zero maneuver & 0.0 & 10.2 & 153.3 & 57.2 \\
\hline 93 & 2 & SMA and Ecc. Maximizer - Initial Peri.: 3.6 [deg] & 2.3 & 10.2 & 153.3 & 57.2 \\
\hline 93 & 3 & SMA Maximizer w/Constant Ecc. & 7.8 & 10.2 & 153.3 & 57.2 \\
\hline 94 & 1 & SMA Maximizer - Initial Peri.: 13.0 [deg] & 4.6 & 9.3 & 152.5 & 53.2 \\
\hline 94 & 2 & SMA Maximizer w/Constant Ecc. & 2.3 & 9.3 & 152.5 & 53.2 \\
\hline 94 & 3 & SMA Maximizer & 2.3 & 9.3 & 152.5 & 53.2 \\
\hline 95 & 1 & SMA Maximizer - Initial Peri.: 231.5 [deg] & 0.0 & 8.6 & 151.0 & 58.2 \\
\hline 95 & 2 & SMA Maximizer - Initial Peri.: 43.5 [deg] & 5.8 & 8.6 & 151.0 & 58.2 \\
\hline 95 & 3 & SMA Maximizer & 2.8 & 8.6 & 151.0 & 58.2 \\
\hline 96 & 1 & SMA Maximizer w/Constant Ecc. & 1.8 & 12.7 & 150.1 & 74.7 \\
\hline 96 & 2 & SMA and Ecc. Maximizer - Initial Peri.: 81.3 [deg] & 9.6 & 12.7 & 150.1 & 74.7 \\
\hline 96 & 3 & SMA Maximizer & 1.2 & 12.7 & 150.1 & 74.7 \\
\hline 97 & 1 & SMA and Ecc. Maximizer - Initial Peri.: 73.3 [deg] & 6.7 & 15.5 & 148.8 & 83.0 \\
\hline 97 & 2 & SMA and Ecc. Maximizer - Initial Peri.: 304.2 [deg] & 8.8 & 15.5 & 148.8 & 83.0 \\
\hline 97 & 3 & Zero maneuver & 0.0 & 15.5 & 148.8 & 83.0 \\
\hline 98 & 1 & Zero maneuver & 0.0 & 8.3 & 148.7 & 50.7 \\
\hline 98 & 2 & Zero maneuver & 0.0 & 8.3 & 148.7 & 50.7 \\
\hline
\end{tabular}




\begin{tabular}{|c|c|c|c|c|c|c|}
\hline Solution & Vehicle & Maneuver & $\begin{array}{l}\text { Propellant } \\
\text { Consumed by } \\
\text { Vehicle [kg] }\end{array}$ & $\begin{array}{c}\text { Propellant } \\
\text { Consumed by } \\
\text { Constellation } \\
{[\mathrm{kg}]}\end{array}$ & $\begin{array}{c}\text { Total } \\
\text { Coverage } \\
\text { Time [hr] } \\
\text { Washington, } \\
\text { D.C. }\end{array}$ & $\begin{array}{c}\text { Total } \\
\text { Coverage } \\
\text { Time [hr] } \\
\text { Montevideo }\end{array}$ \\
\hline 98 & 3 & SMA Maximizer w/Constant Ecc. & 8.3 & 8.3 & 148.7 & 50.7 \\
\hline 99 & 1 & SMA Maximizer w/Constant Ecc. & 2.8 & 12.1 & 148.3 & 73.2 \\
\hline 99 & 2 & SMA Maximizer - Initial Peri.: 32.5 [deg] & 7.8 & 12.1 & 148.3 & 73.2 \\
\hline 99 & 3 & SMA Maximizer & 1.5 & 12.1 & 148.3 & 73.2 \\
\hline 100 & 1 & Zero maneuver & 0.0 & 7.9 & 146.5 & 49.9 \\
\hline 100 & 2 & SMA and Ecc. Maximizer - Initial Peri.: 40.2 [deg] & 0.0 & 7.9 & 146.5 & 49.9 \\
\hline 100 & 3 & SMA Maximizer w/Constant Ecc. & 7.8 & 7.9 & 146.5 & 49.9 \\
\hline 101 & 1 & SMA and Ecc. Maximizer - Initial Peri.: 13.0 [deg] & 9.9 & 23.1 & 145.3 & 102.9 \\
\hline 101 & 2 & SMA Maximizer - Initial Peri.: 6.3 [deg] & 9.4 & 23.1 & 145.3 & 102.9 \\
\hline 101 & 3 & Ecc. Minimizer & 3.8 & 23.1 & 145.3 & 102.9 \\
\hline 102 & 1 & SMA and Ecc. Maximizer - Initial Peri.: 61.4 [deg] & 5.9 & 14.4 & 144.4 & 82.1 \\
\hline 102 & 2 & SMA Maximizer - Initial Peri.: 321.7 [deg] & 8.5 & 14.4 & 144.4 & 82.1 \\
\hline 102 & 3 & Zero maneuver & 0.0 & 14.4 & 144.4 & 82.1 \\
\hline 103 & 1 & SMA Maximizer w/Constant Ecc. & 0.4 & 10.3 & 144.3 & 68.2 \\
\hline 103 & 2 & SMA Maximizer - Initial Peri.: 76.3 [deg] & 8.6 & 10.3 & 144.3 & 68.2 \\
\hline 103 & 3 & SMA Maximizer & 1.2 & 10.3 & 144.3 & 68.2 \\
\hline 104 & 1 & SMA Maximizer w/Constant Ecc. & 4.6 & 13.4 & 144.2 & 76.0 \\
\hline 104 & 2 & SMA Maximizer - Initial Peri.: 297.7 [deg] & 8.7 & 13.4 & 144.2 & 76.0 \\
\hline 104 & 3 & Zero maneuver & 0.0 & 13.4 & 144.2 & 76.0 \\
\hline 105 & 1 & SMA Maximizer w/Constant Ecc. & 0.2 & 9.5 & 142.9 & 67.0 \\
\hline 105 & 2 & SMA Maximizer - Initial Peri.: 32.5 [deg] & 7.8 & 9.5 & 142.9 & 67.0 \\
\hline
\end{tabular}




\begin{tabular}{|c|c|c|c|c|c|c|}
\hline Solution & Vehicle & Maneuver & $\begin{array}{l}\text { Propellant } \\
\text { Consumed by } \\
\text { Vehicle [kg] }\end{array}$ & $\begin{array}{c}\text { Propellant } \\
\text { Consumed by } \\
\text { Constellation } \\
{[\mathrm{kg}]}\end{array}$ & $\begin{array}{c}\text { Total } \\
\text { Coverage } \\
\text { Time [hr] } \\
\text { Washington, } \\
\text { D.C. }\end{array}$ & $\begin{array}{c}\text { Total } \\
\text { Coverage } \\
\text { Time [hr] } \\
\text { Montevideo }\end{array}$ \\
\hline 105 & 3 & SMA Maximizer & 1.5 & 9.5 & 142.9 & 67.0 \\
\hline 106 & 1 & SMA Maximizer w/Constant Ecc. & 0.2 & 7.4 & 140.1 & 53.8 \\
\hline 106 & 2 & SMA Maximizer w/Constant Ecc. & 5.8 & 7.4 & 140.1 & 53.8 \\
\hline 106 & 3 & SMA Maximizer & 1.5 & 7.4 & 140.1 & 53.8 \\
\hline 107 & 1 & SMA Maximizer w/Constant Ecc. & 0.0 & 7.0 & 138.9 & 52.4 \\
\hline 107 & 2 & SMA Maximizer w/Constant Ecc. & 5.5 & 7.0 & 138.9 & 52.4 \\
\hline 107 & 3 & SMA Maximizer & 1.5 & 7.0 & 138.9 & 52.4 \\
\hline 108 & 1 & SMA Maximizer - Initial Peri.: 261.7 [deg] & 0.3 & 9.4 & 138.1 & 64.2 \\
\hline 108 & 2 & SMA Maximizer - Initial Peri.: 143.4 [deg] & 9.1 & 9.4 & 138.1 & 64.2 \\
\hline 108 & 3 & Zero maneuver & 0.0 & 9.4 & 138.1 & 64.2 \\
\hline 109 & 1 & SMA Maximizer - Initial Peri.: 63.5 [deg] & 4.3 & 12.7 & 137.4 & 77.4 \\
\hline 109 & 2 & SMA and Ecc. Maximizer - Initial Peri.: 32.5 [deg] & 7.8 & 12.7 & 137.4 & 77.4 \\
\hline 109 & 3 & Arg.Peri. Maximizer In-Plane Only & 0.5 & 12.7 & 137.4 & 77.4 \\
\hline 110 & 1 & SMA Maximizer w/Constant Ecc. & 0.0 & 6.9 & 136.2 & 57.3 \\
\hline 110 & 2 & SMA Maximizer - Initial Peri.: 32.5 [deg] & 5.4 & 6.9 & 136.2 & 57.3 \\
\hline 110 & 3 & SMA Maximizer & 1.5 & 6.9 & 136.2 & 57.3 \\
\hline 111 & 1 & SMA Maximizer - Initial Peri.: 12.7 [deg] & 0.0 & 8.4 & 135.8 & 60.9 \\
\hline 111 & 2 & SMA Maximizer - Initial Peri.: 143.4 [deg] & 8.4 & 8.4 & 135.8 & 60.9 \\
\hline 111 & 3 & Zero maneuver & 0.0 & 8.4 & 135.8 & 60.9 \\
\hline 112 & 1 & SMA Maximizer w/Constant Ecc. & 5.0 & 11.2 & 135.6 & 70.1 \\
\hline 112 & 2 & SMA Maximizer - Initial Peri.: 69.2 [deg] & 6.2 & 11.2 & 135.6 & 70.1 \\
\hline
\end{tabular}




\begin{tabular}{|c|c|c|c|c|c|c|}
\hline Solution & Vehicle & Maneuver & $\begin{array}{c}\text { Propellant } \\
\text { Consumed by } \\
\text { Vehicle [kg] }\end{array}$ & $\begin{array}{c}\text { Propellant } \\
\text { Consumed by } \\
\text { Constellation } \\
{[\mathrm{kg}]}\end{array}$ & $\begin{array}{c}\text { Total } \\
\text { Coverage } \\
\text { Time [hr] } \\
\text { Washington, } \\
\text { D.C. }\end{array}$ & $\begin{array}{c}\text { Total } \\
\text { Coverage } \\
\text { Time [hr] } \\
\text { Montevideo }\end{array}$ \\
\hline 112 & 3 & Zero maneuver & 0.0 & 11.2 & 135.6 & 70.1 \\
\hline 113 & 1 & Zero maneuver & 0.0 & 10.8 & 135.1 & 72.9 \\
\hline 113 & 2 & SMA Maximizer - Initial Peri.: 50.2 [deg] & 10.0 & 10.8 & 135.1 & 72.9 \\
\hline 113 & 3 & Arg.Peri. Minimizer w/Constant Others & 0.8 & 10.8 & 135.1 & 72.9 \\
\hline 114 & 1 & Zero maneuver & 0.0 & 8.9 & 134.8 & 65.6 \\
\hline 114 & 2 & SMA Maximizer - Initial Peri.: 297.7 [deg] & 8.9 & 8.9 & 134.8 & 65.6 \\
\hline 114 & 3 & Zero maneuver & 0.0 & 8.9 & 134.8 & 65.6 \\
\hline 115 & 1 & Zero maneuver & 0.0 & 8.3 & 132.7 & 63.7 \\
\hline 115 & 2 & SMA Maximizer - Initial Peri.: 297.7 [deg] & 8.3 & 8.3 & 132.7 & 63.7 \\
\hline 115 & 3 & Zero maneuver & 0.0 & 8.3 & 132.7 & 63.7 \\
\hline 116 & 1 & SMA Maximizer - Initial Peri.: 29.2 [deg] & 0.0 & 3.5 & 132.2 & 41.0 \\
\hline 116 & 2 & SMA Maximizer - Initial Peri.: 75.6 [deg] & 1.7 & 3.5 & 132.2 & 41.0 \\
\hline 116 & 3 & SMA Maximizer & 1.8 & 3.5 & 132.2 & 41.0 \\
\hline 117 & 1 & SMA Maximizer - Initial Peri.: 12.7 [deg] & 0.0 & 8.4 & 131.4 & 66.0 \\
\hline 117 & 2 & SMA Maximizer - Initial Peri.: 75.6 [deg] & 8.4 & 8.4 & 131.4 & 66.0 \\
\hline 117 & 3 & Zero maneuver & 0.0 & 8.4 & 131.4 & 66.0 \\
\hline 118 & 1 & Zero maneuver & 0.0 & 8.7 & 131.1 & 68.5 \\
\hline 118 & 2 & SMA Maximizer - Initial Peri.: 56.8 [deg] & 8.7 & 8.7 & 131.1 & 68.5 \\
\hline 118 & 3 & Zero maneuver & 0.0 & 8.7 & 131.1 & 68.5 \\
\hline 119 & 1 & Zero maneuver & 0.0 & 3.4 & 129.5 & 41.2 \\
\hline 119 & 2 & SMA and Ecc. Maximizer - Initial Peri.: 26.6 [deg] & 1.7 & 3.4 & 129.5 & 41.2 \\
\hline
\end{tabular}




\begin{tabular}{|c|c|c|c|c|c|c|}
\hline Solution & Vehicle & Maneuver & $\begin{array}{l}\text { Propellant } \\
\text { Consumed by } \\
\text { Vehicle [kg] }\end{array}$ & $\begin{array}{c}\text { Propellant } \\
\text { Consumed by } \\
\text { Constellation } \\
{[\mathrm{kg}]}\end{array}$ & $\begin{array}{c}\text { Total } \\
\text { Coverage } \\
\text { Time [hr] } \\
\text { Washington, } \\
\text { D.C. }\end{array}$ & $\begin{array}{c}\text { Total } \\
\text { Coverage } \\
\text { Time [hr] } \\
\text { Montevideo }\end{array}$ \\
\hline 119 & 3 & SMA Maximizer & 1.7 & 3.4 & 129.5 & 41.2 \\
\hline 120 & 1 & SMA Minimizer w/Constant Ecc. & 0.1 & 5.6 & 128.8 & 46.8 \\
\hline 120 & 2 & SMA Maximizer w/Constant Ecc. & 3.1 & 5.6 & 128.8 & 46.8 \\
\hline 120 & 3 & SMA Maximizer w/Constant Ecc. & 2.3 & 5.6 & 128.8 & 46.8 \\
\hline 121 & 1 & Zero maneuver & 0.0 & 5.8 & 127.7 & 52.5 \\
\hline 121 & 2 & SMA Maximizer - Initial Peri.: 134.5 [deg] & 5.8 & 5.8 & 127.7 & 52.5 \\
\hline 121 & 3 & Zero maneuver & 0.0 & 5.8 & 127.7 & 52.5 \\
\hline 122 & 1 & Zero maneuver & 0.0 & 6.2 & 127.1 & 55.4 \\
\hline 122 & 2 & SMA Maximizer w/Constant Ecc. & 6.2 & 6.2 & 127.1 & 55.4 \\
\hline 122 & 3 & Zero maneuver & 0.0 & 6.2 & 127.1 & 55.4 \\
\hline 123 & 1 & SMA and Ecc. Maximizer - Initial Peri.: 13.0 [deg] & 0.0 & 5.4 & 124.9 & 51.5 \\
\hline 123 & 2 & SMA Maximizer w/Constant Ecc. & 5.4 & 5.4 & 124.9 & 51.5 \\
\hline 123 & 3 & Zero maneuver & 0.0 & 5.4 & 124.9 & 51.5 \\
\hline 124 & 1 & SMA and Ecc. Maximizer - Initial Peri.: 13.0 [deg] & 0.0 & 5.1 & 124.3 & 50.3 \\
\hline 124 & 2 & SMA Maximizer w/Constant Ecc. & 5.1 & 5.1 & 124.3 & 50.3 \\
\hline 124 & 3 & Zero maneuver & 0.0 & 5.1 & 124.3 & 50.3 \\
\hline 125 & 1 & Zero maneuver & 0.0 & 3.5 & 123.5 & 41.9 \\
\hline 125 & 2 & SMA Maximizer - Initial Peri.: 109.1 [deg] & 1.2 & 3.5 & 123.5 & 41.9 \\
\hline 125 & 3 & SMA Maximizer w/Constant Ecc. & 2.3 & 3.5 & 123.5 & 41.9 \\
\hline 126 & 1 & Zero maneuver & 0.0 & 5.9 & 123.4 & 58.5 \\
\hline 126 & 2 & SMA and Ecc. Maximizer - Initial Peri.: 351.0 [deg] & 5.9 & 5.9 & 123.4 & 58.5 \\
\hline
\end{tabular}




\begin{tabular}{|c|c|c|c|c|c|c|}
\hline Solution & Vehicle & Maneuver & $\begin{array}{l}\text { Propellant } \\
\text { Consumed by } \\
\text { Vehicle [kg] }\end{array}$ & $\begin{array}{c}\text { Propellant } \\
\text { Consumed by } \\
\text { Constellation } \\
{[\mathrm{kg}]}\end{array}$ & $\begin{array}{c}\text { Total } \\
\text { Coverage } \\
\text { Time [hr] } \\
\text { Washington, } \\
\text { D.C. }\end{array}$ & $\begin{array}{c}\text { Total } \\
\text { Coverage } \\
\text { Time [hr] } \\
\text { Montevideo }\end{array}$ \\
\hline 126 & 3 & Incl. Minimizer - Initial RAAN: 135.2 [deg] & 0.1 & 5.9 & 123.4 & 58.5 \\
\hline 127 & 1 & Zero maneuver & 0.0 & 1.5 & 122.5 & 36.5 \\
\hline 127 & 2 & Zero maneuver & 0.0 & 1.5 & 122.5 & 36.5 \\
\hline 127 & 3 & SMA Maximizer & 1.5 & 1.5 & 122.5 & 36.5 \\
\hline 128 & 1 & Zero maneuver & 0.0 & 3.4 & 122.2 & 42.2 \\
\hline 128 & 2 & SMA Maximizer - Initial Peri.: 45.9 [deg] & 1.1 & 3.4 & 122.2 & 42.2 \\
\hline 128 & 3 & SMA Maximizer w/Constant Ecc. & 2.3 & 3.4 & 122.2 & 42.2 \\
\hline 129 & 1 & Zero maneuver & 0.0 & 3.1 & 120.4 & 41.9 \\
\hline 129 & 2 & SMA Maximizer - Initial Peri.: 45.9 [deg] & 1.1 & 3.1 & 120.4 & 41.9 \\
\hline 129 & 3 & SMA Maximizer w/Constant Ecc. & 2.0 & 3.1 & 120.4 & 41.9 \\
\hline 130 & 1 & SMA Maximizer - Initial Peri.: 284.8 [deg] & 1.5 & 4.2 & 120.2 & 45.4 \\
\hline 130 & 2 & SMA Maximizer - Initial Peri.: 148.8 [deg] & 2.7 & 4.2 & 120.2 & 45.4 \\
\hline 130 & 3 & Zero maneuver & 0.0 & 4.2 & 120.2 & 45.4 \\
\hline 131 & 1 & SMA Maximizer w/Constant Ecc. & 1.8 & 4.4 & 119.9 & 47.0 \\
\hline 131 & 2 & SMA Maximizer - Initial Peri.: 73.5 [deg] & 2.6 & 4.4 & 119.9 & 47.0 \\
\hline 131 & 3 & Zero maneuver & 0.0 & 4.4 & 119.9 & 47.0 \\
\hline 132 & 1 & Zero maneuver & 0.0 & 3.1 & 119.4 & 42.6 \\
\hline 132 & 2 & SMA Maximizer - Initial Peri.: 143.4 [deg] & 3.1 & 3.1 & 119.4 & 42.6 \\
\hline 132 & 3 & Zero maneuver & 0.0 & 3.1 & 119.4 & 42.6 \\
\hline 133 & 1 & Zero maneuver & 0.0 & 4.0 & 117.9 & 44.7 \\
\hline 133 & 2 & SMA Maximizer - Initial Peri.: 266.1 [deg] & 3.2 & 4.0 & 117.9 & 44.7 \\
\hline
\end{tabular}




\begin{tabular}{|c|c|c|c|c|c|c|}
\hline Solution & Vehicle & Maneuver & $\begin{array}{l}\text { Propellant } \\
\text { Consumed by } \\
\text { Vehicle [kg] }\end{array}$ & $\begin{array}{c}\text { Propellant } \\
\text { Consumed by } \\
\text { Constellation } \\
{[\mathrm{kg}]}\end{array}$ & $\begin{array}{c}\text { Total } \\
\text { Coverage } \\
\text { Time [hr] } \\
\text { Washington, } \\
\text { D.C. }\end{array}$ & $\begin{array}{c}\text { Total } \\
\text { Coverage } \\
\text { Time [hr] } \\
\text { Montevideo }\end{array}$ \\
\hline 133 & 3 & Arg.Peri. Minimizer w/Constant Others & 0.8 & 4.0 & 117.9 & 44.7 \\
\hline 134 & 1 & Zero maneuver & 0.0 & 2.9 & 116.2 & 45.0 \\
\hline 134 & 2 & SMA Maximizer - Initial Peri.: 358.8 [deg] & 2.9 & 2.9 & 116.2 & 45.0 \\
\hline 134 & 3 & Zero maneuver & 0.0 & 2.9 & 116.2 & 45.0 \\
\hline 135 & 1 & Zero maneuver & 0.0 & 2.2 & 115.4 & 42.0 \\
\hline 135 & 2 & SMA Maximizer - Initial Peri.: 69.2 [deg] & 2.2 & 2.2 & 115.4 & 42.0 \\
\hline 135 & 3 & Zero maneuver & 0.0 & 2.2 & 115.4 & 42.0 \\
\hline 136 & 1 & Zero maneuver & 0.0 & 2.3 & 114.4 & 43.1 \\
\hline 136 & 2 & SMA and Ecc. Maximizer - Initial Peri.: 3.6 [deg] & 2.3 & 2.3 & 114.4 & 43.1 \\
\hline 136 & 3 & Zero maneuver & 0.0 & 2.3 & 114.4 & 43.1 \\
\hline 137 & 1 & SMA Maximizer w/Constant Ecc. & 0.3 & 1.5 & 112.3 & 39.8 \\
\hline 137 & 2 & SMA Maximizer - Initial Peri.: 352.8 [deg] & 1.2 & 1.5 & 112.3 & 39.8 \\
\hline 137 & 3 & Zero maneuver & 0.0 & 1.5 & 112.3 & 39.8 \\
\hline 138 & 1 & Zero maneuver & 0.0 & 1.1 & 111.6 & 38.8 \\
\hline 138 & 2 & SMA Maximizer - Initial Peri.: 27.2 [deg] & 1.1 & 1.1 & 111.6 & 38.8 \\
\hline 138 & 3 & Zero maneuver & 0.0 & 1.1 & 111.6 & 38.8 \\
\hline 139 & 1 & Zero maneuver & 0.0 & 1.0 & 111.1 & 38.3 \\
\hline 139 & 2 & SMA Maximizer - Initial Peri.: 37.0 [deg] & 1.0 & 1.0 & 111.1 & 38.3 \\
\hline 139 & 3 & Zero maneuver & 0.0 & 1.0 & 111.1 & 38.3 \\
\hline 140 & 1 & Zero maneuver & 0.0 & 0.6 & 110.7 & 37.3 \\
\hline 140 & 2 & SMA and Ecc. Maximizer - Initial Peri.: 100.4 [deg] & 0.6 & 0.6 & 110.7 & 37.3 \\
\hline
\end{tabular}




\begin{tabular}{|c|c|c|c|c|c|c|}
\hline Solution & Vehicle & Maneuver & $\begin{array}{l}\text { Propellant } \\
\text { Consumed by } \\
\text { Vehicle [kg] }\end{array}$ & $\begin{array}{c}\text { Propellant } \\
\text { Consumed by } \\
\text { Constellation } \\
{[\mathrm{kg}]}\end{array}$ & $\begin{array}{c}\text { Total } \\
\text { Coverage } \\
\text { Time [hr] } \\
\text { Washington, } \\
\text { D.C. }\end{array}$ & $\begin{array}{c}\text { Total } \\
\text { Coverage } \\
\text { Time [hr] } \\
\text { Montevideo }\end{array}$ \\
\hline 140 & 3 & Zero maneuver & 0.0 & 0.6 & 110.7 & 37.3 \\
\hline 141 & 1 & Zero maneuver & 0.0 & 0.7 & 110.5 & 37.5 \\
\hline 141 & 2 & SMA Maximizer - Initial Peri.: 56.8 [deg] & 0.7 & 0.7 & 110.5 & 37.5 \\
\hline 141 & 3 & Zero maneuver & 0.0 & 0.7 & 110.5 & 37.5 \\
\hline 142 & 1 & Zero maneuver & 0.0 & 0.0 & 108.0 & 36.0 \\
\hline 142 & 2 & Zero maneuver & 0.0 & 0.0 & 108.0 & 36.0 \\
\hline 142 & 3 & Zero maneuver & 0.0 & 0.0 & 108.0 & 36.0 \\
\hline
\end{tabular}


Table 20: Final nondominated surface for Example 6.4

\begin{tabular}{|c|c|c|c|c|c|c|}
\hline Solution & Vehicle & Maneuver & $\begin{array}{c}\text { Propellant } \\
\text { Consumed by } \\
\text { Vehicle [kg] }\end{array}$ & $\begin{array}{c}\text { Propellant } \\
\text { Consumed by } \\
\text { Constellation } \\
{[\mathrm{kg}]}\end{array}$ & $\begin{array}{c}\text { Total } \\
\text { Coverage } \\
\text { Time [hr] } \\
\text { Washington, } \\
\text { D.C. }\end{array}$ & $\begin{array}{c}\text { Total } \\
\text { Coverage } \\
\text { Time [hr] } \\
\text { Montevideo }\end{array}$ \\
\hline 1 & 1 & SMA and Ecc. Maximizer - Initial Peri.: 356.4 [deg] & 10.0 & 20.5 & 169.9 & 90.4 \\
\hline 1 & 2 & SMA and Ecc. Maximizer - Initial Peri.: 199.1 [deg] & 10.0 & 20.5 & 169.9 & 90.4 \\
\hline 1 & 3 & SMA and Ecc. Maximizer & 0.5 & 20.5 & 169.9 & 90.4 \\
\hline 2 & 1 & SMA and Ecc. Maximizer - Initial Peri.: 104.9 [deg] & 10.0 & 20.2 & 168.6 & 90.2 \\
\hline 2 & 2 & SMA and Ecc. Maximizer - Initial Peri.: 199.1 [deg] & 10.0 & 20.2 & 168.6 & 90.2 \\
\hline 2 & 3 & SMA and Ecc. Maximizer & 0.2 & 20.2 & 168.6 & 90.2 \\
\hline 3 & 1 & SMA Maximizer - Initial Peri.: 37.9 [deg] & 10.0 & 20.7 & 168.6 & 91.1 \\
\hline 3 & 2 & SMA Maximizer - Initial Peri.: 222.1 [deg] & 10.0 & 20.7 & 168.6 & 91.1 \\
\hline 3 & 3 & SMA Maximizer w/Constant Ecc. & 0.7 & 20.7 & 168.6 & 91.1 \\
\hline 4 & 1 & SMA Maximizer - Initial Peri.: 323.2 [deg] & 9.9 & 20.4 & 167.6 & 92.3 \\
\hline 4 & 2 & SMA and Ecc. Maximizer - Initial Peri.: 143.7 [deg] & 10.0 & 20.4 & 167.6 & 92.3 \\
\hline 4 & 3 & SMA Maximizer & 0.5 & 20.4 & 167.6 & 92.3 \\
\hline 5 & 1 & SMA and Ecc. Maximizer - Initial Peri.: 251.6 [deg] & 10.0 & 19.6 & 166.5 & 87.4 \\
\hline 5 & 2 & SMA and Ecc. Maximizer - Initial Peri.: 176.1 [deg] & 9.1 & 19.6 & 166.5 & 87.4 \\
\hline 5 & 3 & SMA Maximizer & 0.5 & 19.6 & 166.5 & 87.4 \\
\hline 6 & 1 & SMA and Ecc. Maximizer - Initial Peri.: 35.7 [deg] & 10.0 & 19.9 & 166.1 & 88.0 \\
\hline 6 & 2 & SMA and Ecc. Maximizer - Initial Peri.: 176.1 [deg] & 9.1 & 19.9 & 166.1 & 88.0 \\
\hline 6 & 3 & SMA Maximizer w/Constant Ecc. & 0.7 & 19.9 & 166.1 & 88.0 \\
\hline 7 & 1 & SMA and Ecc. Maximizer - Initial Peri.: 332.4 [deg] & 10.0 & 20.5 & 166.0 & 95.5 \\
\hline 7 & 2 & SMA Maximizer w/Constant Ecc. & 10.0 & 20.5 & 166.0 & 95.5 \\
\hline
\end{tabular}




\begin{tabular}{|c|c|c|c|c|c|c|}
\hline Solution & Vehicle & Maneuver & $\begin{array}{c}\text { Propellant } \\
\text { Consumed by } \\
\text { Vehicle [kg] }\end{array}$ & $\begin{array}{c}\text { Propellant } \\
\text { Consumed by } \\
\text { Constellation } \\
\text { [kg] }\end{array}$ & $\begin{array}{c}\text { Total } \\
\text { Coverage } \\
\text { Time [hr] } \\
\text { Washington, } \\
\text { D.C. }\end{array}$ & $\begin{array}{c}\text { Total } \\
\text { Coverage } \\
\text { Time [hr] } \\
\text { Montevideo }\end{array}$ \\
\hline 7 & 3 & SMA and Ecc. Maximizer & 0.5 & 20.5 & 166.0 & 95.5 \\
\hline 8 & 1 & SMA Maximizer - Initial Peri.: 147.8 [deg] & 10.0 & 20.5 & 165.9 & 96.2 \\
\hline 8 & 2 & SMA Maximizer w/Constant Ecc. & 10.0 & 20.5 & 165.9 & 96.2 \\
\hline 8 & 3 & SMA and Ecc. Maximizer & 0.5 & 20.5 & 165.9 & 96.2 \\
\hline 9 & 1 & SMA Maximizer - Initial Peri.: 327.4 [deg] & 9.8 & 19.4 & 165.0 & 89.0 \\
\hline 9 & 2 & SMA and Ecc. Maximizer - Initial Peri.: 143.7 [deg] & 9.1 & 19.4 & 165.0 & 89.0 \\
\hline 9 & 3 & SMA Maximizer & 0.5 & 19.4 & 165.0 & 89.0 \\
\hline 10 & 1 & SMA and Ecc. Maximizer - Initial Peri.: 89.8 [deg] & 10.0 & 20.7 & 164.2 & 96.4 \\
\hline 10 & 2 & SMA and Ecc. Maximizer - Initial Peri.: 117.0 [deg] & 10.0 & 20.7 & 164.2 & 96.4 \\
\hline 10 & 3 & SMA Maximizer w/Constant Ecc. & 0.7 & 20.7 & 164.2 & 96.4 \\
\hline 11 & 1 & SMA and Ecc. Maximizer - Initial Peri.: 104.9 [deg] & 10.0 & 20.5 & 164.2 & 98.1 \\
\hline 11 & 2 & SMA Maximizer - Initial Peri.: 332.3 [deg] & 10.0 & 20.5 & 164.2 & 98.1 \\
\hline 11 & 3 & SMA Maximizer & 0.5 & 20.5 & 164.2 & 98.1 \\
\hline 12 & 1 & SMA Maximizer - Initial Peri.: 332.4 [deg] & 10.0 & 19.4 & 162.9 & 91.6 \\
\hline 12 & 2 & SMA Maximizer w/Constant Ecc. & 8.9 & 19.4 & 162.9 & 91.6 \\
\hline 12 & 3 & SMA and Ecc. Maximizer & 0.5 & 19.4 & 162.9 & 91.6 \\
\hline 13 & 1 & SMA Maximizer - Initial Peri.: 75.1 [deg] & 10.0 & 20.5 & 162.2 & 100.0 \\
\hline 13 & 2 & SMA and Ecc. Maximizer - Initial Peri.: 19.9 [deg] & 10.0 & 20.5 & 162.2 & 100.0 \\
\hline 13 & 3 & SMA and Ecc. Maximizer & 0.5 & 20.5 & 162.2 & 100.0 \\
\hline 14 & 1 & SMA Maximizer - Initial Peri.: 104.9 [deg] & 9.9 & 20.4 & 161.7 & 99.9 \\
\hline 14 & 2 & SMA and Ecc. Maximizer - Initial Peri.: 7.9 [deg] & 10.0 & 20.4 & 161.7 & 99.9 \\
\hline
\end{tabular}




\begin{tabular}{|c|c|c|c|c|c|c|}
\hline Solution & Vehicle & Maneuver & $\begin{array}{l}\text { Propellant } \\
\text { Consumed by } \\
\text { Vehicle [kg] }\end{array}$ & $\begin{array}{c}\text { Propellant } \\
\text { Consumed by } \\
\text { Constellation } \\
\text { [kg] }\end{array}$ & $\begin{array}{c}\text { Total } \\
\text { Coverage } \\
\text { Time [hr] } \\
\text { Washington, } \\
\text { D.C. }\end{array}$ & $\begin{array}{c}\text { Total } \\
\text { Coverage } \\
\text { Time [hr] } \\
\text { Montevideo }\end{array}$ \\
\hline 14 & 3 & SMA Maximizer & 0.5 & 20.4 & 161.7 & 99.9 \\
\hline 15 & 1 & SMA and Ecc. Maximizer - Initial Peri.: 332.4 [deg] & 8.1 & 17.5 & 160.9 & 82.1 \\
\hline 15 & 2 & SMA and Ecc. Maximizer - Initial Peri.: 199.1 [deg] & 8.9 & 17.5 & 160.9 & 82.1 \\
\hline 15 & 3 & SMA Maximizer & 0.5 & 17.5 & 160.9 & 82.1 \\
\hline 16 & 1 & SMA and Ecc. Maximizer - Initial Peri.: 221.3 [deg] & 9.9 & 18.9 & 160.6 & 89.7 \\
\hline 16 & 2 & SMA Maximizer w/Constant Ecc. & 8.5 & 18.9 & 160.6 & 89.7 \\
\hline 16 & 3 & SMA Maximizer & 0.5 & 18.9 & 160.6 & 89.7 \\
\hline 17 & 1 & SMA Maximizer - Initial Peri.: 221.3 [deg] & 9.7 & 18.3 & 159.7 & 87.6 \\
\hline 17 & 2 & SMA Maximizer w/Constant Ecc. & 8.2 & 18.3 & 159.7 & 87.6 \\
\hline 17 & 3 & SMA and Ecc. Maximizer & 0.5 & 18.3 & 159.7 & 87.6 \\
\hline 18 & 1 & SMA Maximizer - Initial Peri.: 313.1 [deg] & 10.0 & 18.9 & 159.3 & 90.1 \\
\hline 18 & 2 & SMA and Ecc. Maximizer - Initial Peri.: 124.6 [deg] & 8.9 & 18.9 & 159.3 & 90.1 \\
\hline 18 & 3 & Ecc. Maximizer & 0.0 & 18.9 & 159.3 & 90.1 \\
\hline 19 & 1 & SMA Maximizer - Initial Peri.: 16.4 [deg] & 9.5 & 18.6 & 158.4 & 89.5 \\
\hline 19 & 2 & SMA and Ecc. Maximizer - Initial Peri.: 131.1 [deg] & 9.1 & 18.6 & 158.4 & 89.5 \\
\hline 19 & 3 & Ecc. Maximizer & 0.0 & 18.6 & 158.4 & 89.5 \\
\hline 20 & 1 & SMA Maximizer - Initial Peri.: 40.0 [deg] & 9.7 & 19.1 & 158.3 & 95.4 \\
\hline 20 & 2 & SMA and Ecc. Maximizer - Initial Peri.: 22.4 [deg] & 8.9 & 19.1 & 158.3 & 95.4 \\
\hline 20 & 3 & SMA and Ecc. Maximizer & 0.5 & 19.1 & 158.3 & 95.4 \\
\hline 21 & 1 & SMA Maximizer - Initial Peri.: 40.0 [deg] & 9.7 & 17.6 & 158.1 & 85.6 \\
\hline 21 & 2 & SMA Maximizer w/Constant Ecc. & 7.4 & 17.6 & 158.1 & 85.6 \\
\hline
\end{tabular}




\begin{tabular}{|c|c|c|c|c|c|c|}
\hline Solution & Vehicle & Maneuver & $\begin{array}{c}\text { Propellant } \\
\text { Consumed by } \\
\text { Vehicle [kg] }\end{array}$ & $\begin{array}{c}\text { Propellant } \\
\text { Consumed by } \\
\text { Constellation } \\
{[\mathrm{kg}]}\end{array}$ & $\begin{array}{c}\text { Total } \\
\text { Coverage } \\
\text { Time [hr] } \\
\text { Washington, } \\
\text { D.C. }\end{array}$ & $\begin{array}{c}\text { Total } \\
\text { Coverage } \\
\text { Time [hr] } \\
\text { Montevideo }\end{array}$ \\
\hline 21 & 3 & SMA and Ecc. Maximizer & 0.5 & 17.6 & 158.1 & 85.6 \\
\hline 22 & 1 & SMA Maximizer - Initial Peri.: 104.9 [deg] & 6.2 & 16.9 & 157.6 & 82.4 \\
\hline 22 & 2 & SMA Maximizer - Initial Peri.: 247.7 [deg] & 10.0 & 16.9 & 157.6 & 82.4 \\
\hline 22 & 3 & SMA Maximizer w/Constant Ecc. & 0.7 & 16.9 & 157.6 & 82.4 \\
\hline 23 & 1 & SMA Maximizer - Initial Peri.: 75.1 [deg] & 9.7 & 19.6 & 157.3 & 97.4 \\
\hline 23 & 2 & SMA and Ecc. Maximizer - Initial Peri.: 37.2 [deg] & 9.1 & 19.6 & 157.3 & 97.4 \\
\hline 23 & 3 & SMA Maximizer w/Constant Ecc. & 0.7 & 19.6 & 157.3 & 97.4 \\
\hline 24 & 1 & SMA Maximizer - Initial Peri.: 323.2 [deg] & 9.9 & 19.6 & 156.9 & 98.0 \\
\hline 24 & 2 & SMA and Ecc. Maximizer - Initial Peri.: 7.1 [deg] & 9.7 & 19.6 & 156.9 & 98.0 \\
\hline 24 & 3 & SMA and Ecc. Maximizer & 0.0 & 19.6 & 156.9 & 98.0 \\
\hline 25 & 1 & SMA Maximizer - Initial Peri.: 75.1 [deg] & 9.7 & 18.6 & 156.8 & 91.3 \\
\hline 25 & 2 & SMA and Ecc. Maximizer - Initial Peri.: 77.2 [deg] & 8.2 & 18.6 & 156.8 & 91.3 \\
\hline 25 & 3 & SMA Maximizer w/Constant Ecc. & 0.7 & 18.6 & 156.8 & 91.3 \\
\hline 26 & 1 & SMA Maximizer - Initial Peri.: 37.9 [deg] & 6.2 & 16.9 & 156.3 & 83.5 \\
\hline 26 & 2 & SMA and Ecc. Maximizer - Initial Peri.: 247.7 [deg] & 10.0 & 16.9 & 156.3 & 83.5 \\
\hline 26 & 3 & SMA Maximizer w/Constant Ecc. & 0.7 & 16.9 & 156.3 & 83.5 \\
\hline 27 & 1 & SMA Maximizer - Initial Peri.: 75.1 [deg] & 9.7 & 18.3 & 155.4 & 92.6 \\
\hline 27 & 2 & SMA and Ecc. Maximizer - Initial Peri.: 19.9 [deg] & 8.2 & 18.3 & 155.4 & 92.6 \\
\hline 27 & 3 & SMA and Ecc. Maximizer & 0.5 & 18.3 & 155.4 & 92.6 \\
\hline 28 & 1 & SMA and Ecc. Maximizer - Initial Peri.: 323.2 [deg] & 9.9 & 15.9 & 154.9 & 76.0 \\
\hline 28 & 2 & SMA and Ecc. Maximizer - Initial Peri.: 191.1 [deg] & 5.5 & 15.9 & 154.9 & 76.0 \\
\hline
\end{tabular}




\begin{tabular}{|c|c|c|c|c|c|c|}
\hline Solution & Vehicle & Maneuver & $\begin{array}{l}\text { Propellant } \\
\text { Consumed by } \\
\text { Vehicle [kg] }\end{array}$ & $\begin{array}{c}\text { Propellant } \\
\text { Consumed by } \\
\text { Constellation } \\
{[\mathrm{kg}]}\end{array}$ & $\begin{array}{c}\text { Total } \\
\text { Coverage } \\
\text { Time [hr] } \\
\text { Washington, } \\
\text { D.C. }\end{array}$ & $\begin{array}{c}\text { Total } \\
\text { Coverage } \\
\text { Time [hr] } \\
\text { Montevideo }\end{array}$ \\
\hline 28 & 3 & SMA Maximizer & 0.5 & 15.9 & 154.9 & 76.0 \\
\hline 29 & 1 & SMA Maximizer - Initial Peri.: 104.9 [deg] & 10.0 & 21.2 & 154.6 & 101.0 \\
\hline 29 & 2 & SMA and Ecc. Maximizer - Initial Peri.: 7.9 [deg] & 9.9 & 21.2 & 154.6 & 101.0 \\
\hline 29 & 3 & Ecc. Minimizer & 1.2 & 21.2 & 154.6 & 101.0 \\
\hline 30 & 1 & SMA and Ecc. Maximizer - Initial Peri.: 104.9 [deg] & 8.1 & 15.8 & 154.1 & 76.9 \\
\hline 30 & 2 & SMA Maximizer - Initial Peri.: 178.2 [deg] & 7.4 & 15.8 & 154.1 & 76.9 \\
\hline 30 & 3 & SMA and Ecc. Maximizer & 0.2 & 15.8 & 154.1 & 76.9 \\
\hline 31 & 1 & SMA and Ecc. Maximizer - Initial Peri.: 130.8 [deg] & 3.1 & 13.6 & 153.6 & 71.5 \\
\hline 31 & 2 & SMA and Ecc. Maximizer - Initial Peri.: 199.1 [deg] & 10.0 & 13.6 & 153.6 & 71.5 \\
\hline 31 & 3 & SMA Maximizer & 0.5 & 13.6 & 153.6 & 71.5 \\
\hline 32 & 1 & SMA Maximizer - Initial Peri.: 327.4 [deg] & 9.9 & 16.2 & 153.5 & 78.9 \\
\hline 32 & 2 & SMA Maximizer - Initial Peri.: 264.8 [deg] & 5.8 & 16.2 & 153.5 & 78.9 \\
\hline 32 & 3 & SMA and Ecc. Maximizer & 0.5 & 16.2 & 153.5 & 78.9 \\
\hline 33 & 1 & SMA Maximizer w/Constant Ecc. & 3.1 & 13.6 & 152.8 & 71.8 \\
\hline 33 & 2 & SMA and Ecc. Maximizer - Initial Peri.: 199.1 [deg] & 10.0 & 13.6 & 152.8 & 71.8 \\
\hline 33 & 3 & SMA and Ecc. Maximizer & 0.5 & 13.6 & 152.8 & 71.8 \\
\hline 34 & 1 & SMA and Ecc. Maximizer - Initial Peri.: 130.8 [deg] & 2.6 & 13.1 & 152.1 & 70.2 \\
\hline 34 & 2 & SMA and Ecc. Maximizer - Initial Peri.: 190.6 [deg] & 10.0 & 13.1 & 152.1 & 70.2 \\
\hline 34 & 3 & SMA Maximizer & 0.5 & 13.1 & 152.1 & 70.2 \\
\hline 35 & 1 & SMA Maximizer - Initial Peri.: 75.1 [deg] & 9.7 & 17.8 & 151.6 & 92.7 \\
\hline 35 & 2 & SMA and Ecc. Maximizer - Initial Peri.: 19.9 [deg] & 8.2 & 17.8 & 151.6 & 92.7 \\
\hline
\end{tabular}




\begin{tabular}{|c|c|c|c|c|c|c|}
\hline Solution & Vehicle & Maneuver & $\begin{array}{c}\text { Propellant } \\
\text { Consumed by } \\
\text { Vehicle [kg] }\end{array}$ & $\begin{array}{c}\text { Propellant } \\
\text { Consumed by } \\
\text { Constellation } \\
\text { [kg] }\end{array}$ & $\begin{array}{c}\text { Total } \\
\text { Coverage } \\
\text { Time [hr] } \\
\text { Washington, } \\
\text { D.C. }\end{array}$ & $\begin{array}{c}\text { Total } \\
\text { Coverage } \\
\text { Time [hr] } \\
\text { Montevideo }\end{array}$ \\
\hline 35 & 3 & Zero maneuver & 0.0 & 17.8 & 151.6 & 92.7 \\
\hline 36 & 1 & SMA Maximizer w/Constant Ecc. & 4.8 & 13.9 & 151.4 & 72.1 \\
\hline 36 & 2 & SMA Maximizer - Initial Peri.: 166.2 [deg] & 8.7 & 13.9 & 151.4 & 72.1 \\
\hline 36 & 3 & SMA and Ecc. Maximizer & 0.5 & 13.9 & 151.4 & 72.1 \\
\hline 37 & 1 & SMA and Ecc. Maximizer - Initial Peri.: 128.5 [deg] & 9.7 & 17.8 & 151.4 & 92.9 \\
\hline 37 & 2 & SMA and Ecc. Maximizer - Initial Peri.: 19.9 [deg] & 8.2 & 17.8 & 151.4 & 92.9 \\
\hline 37 & 3 & SMA and Ecc. Maximizer & 0.0 & 17.8 & 151.4 & 92.9 \\
\hline 38 & 1 & SMA and Ecc. Maximizer - Initial Peri.: 251.7 [deg] & 9.9 & 16.3 & 151.3 & 83.5 \\
\hline 38 & 2 & SMA and Ecc. Maximizer - Initial Peri.: 332.3 [deg] & 6.0 & 16.3 & 151.3 & 83.5 \\
\hline 38 & 3 & SMA Maximizer & 0.5 & 16.3 & 151.3 & 83.5 \\
\hline 39 & 1 & SMA Maximizer - Initial Peri.: 37.9 [deg] & 3.1 & 13.3 & 151.1 & 72.2 \\
\hline 39 & 2 & SMA and Ecc. Maximizer - Initial Peri.: 199.1 [deg] & 10.0 & 13.3 & 151.1 & 72.2 \\
\hline 39 & 3 & SMA and Ecc. Maximizer & 0.2 & 13.3 & 151.1 & 72.2 \\
\hline 40 & 1 & SMA Maximizer - Initial Peri.: 106.4 [deg] & 6.2 & 15.4 & 151.0 & 80.3 \\
\hline 40 & 2 & SMA and Ecc. Maximizer - Initial Peri.: 284.1 [deg] & 8.5 & 15.4 & 151.0 & 80.3 \\
\hline 40 & 3 & SMA Maximizer w/Constant Ecc. & 0.7 & 15.4 & 151.0 & 80.3 \\
\hline 41 & 1 & SMA and Ecc. Maximizer - Initial Peri.: 254.2 [deg] & 5.9 & 14.3 & 150.6 & 76.1 \\
\hline 41 & 2 & SMA Maximizer w/Constant Ecc. & 7.9 & 14.3 & 150.6 & 76.1 \\
\hline 41 & 3 & SMA and Ecc. Maximizer & 0.5 & 14.3 & 150.6 & 76.1 \\
\hline 42 & 1 & SMA Maximizer - Initial Peri.: 75.1 [deg] & 6.2 & 15.8 & 149.9 & 86.5 \\
\hline 42 & 2 & SMA and Ecc. Maximizer - Initial Peri.: 349.0 [deg] & 9.1 & 15.8 & 149.9 & 86.5 \\
\hline
\end{tabular}




\begin{tabular}{|c|c|c|c|c|c|c|}
\hline Solution & Vehicle & Maneuver & $\begin{array}{l}\text { Propellant } \\
\text { Consumed by } \\
\text { Vehicle [kg] }\end{array}$ & $\begin{array}{c}\text { Propellant } \\
\text { Consumed by } \\
\text { Constellation } \\
{[\mathrm{kg}]}\end{array}$ & $\begin{array}{c}\text { Total } \\
\text { Coverage } \\
\text { Time [hr] } \\
\text { Washington, } \\
\text { D.C. }\end{array}$ & $\begin{array}{c}\text { Total } \\
\text { Coverage } \\
\text { Time [hr] } \\
\text { Montevideo }\end{array}$ \\
\hline 42 & 3 & SMA and Ecc. Maximizer & 0.5 & 15.8 & 149.9 & 86.5 \\
\hline 43 & 1 & SMA Maximizer - Initial Peri.: 327.4 [deg] & 3.1 & 13.4 & 149.3 & 74.3 \\
\hline 43 & 2 & SMA Maximizer - Initial Peri.: 264.8 [deg] & 9.8 & 13.4 & 149.3 & 74.3 \\
\hline 43 & 3 & SMA and Ecc. Maximizer & 0.5 & 13.4 & 149.3 & 74.3 \\
\hline 44 & 1 & SMA Maximizer - Initial Peri.: 251.7 [deg] & 6.1 & 14.8 & 148.0 & 81.3 \\
\hline 44 & 2 & SMA and Ecc. Maximizer - Initial Peri.: 349.0 [deg] & 8.2 & 14.8 & 148.0 & 81.3 \\
\hline 44 & 3 & SMA and Ecc. Maximizer & 0.5 & 14.8 & 148.0 & 81.3 \\
\hline 45 & 1 & SMA Maximizer - Initial Peri.: 104.9 [deg] & 8.1 & 16.3 & 147.9 & 89.0 \\
\hline 45 & 2 & SMA Maximizer - Initial Peri.: 19.9 [deg] & 8.2 & 16.3 & 147.9 & 89.0 \\
\hline 45 & 3 & SMA Maximizer & 0.0 & 16.3 & 147.9 & 89.0 \\
\hline 46 & 1 & SMA Maximizer - Initial Peri.: 330.2 [deg] & 6.0 & 15.2 & 147.1 & 85.3 \\
\hline 46 & 2 & SMA and Ecc. Maximizer - Initial Peri.: 354.8 [deg] & 8.8 & 15.2 & 147.1 & 85.3 \\
\hline 46 & 3 & SMA and Ecc. Maximizer & 0.5 & 15.2 & 147.1 & 85.3 \\
\hline 47 & 1 & SMA and Ecc. Maximizer - Initial Peri.: 357.8 [deg] & 6.2 & 15.1 & 146.9 & 83.2 \\
\hline 47 & 2 & SMA and Ecc. Maximizer - Initial Peri.: 76.2 [deg] & 8.4 & 15.1 & 146.9 & 83.2 \\
\hline 47 & 3 & SMA and Ecc. Maximizer & 0.5 & 15.1 & 146.9 & 83.2 \\
\hline 48 & 1 & SMA and Ecc. Maximizer - Initial Peri.: 352.4 [deg] & 10.0 & 24.9 & 146.9 & 103.4 \\
\hline 48 & 2 & SMA and Ecc. Maximizer - Initial Peri.: 310.2 [deg] & 10.0 & 24.9 & 146.9 & 103.4 \\
\hline 48 & 3 & Ecc. Minimizer & 4.9 & 24.9 & 146.9 & 103.4 \\
\hline 49 & 1 & SMA and Ecc. Maximizer - Initial Peri.: 313.0 [deg] & 9.9 & 24.0 & 146.5 & 103.9 \\
\hline 49 & 2 & SMA Maximizer - Initial Peri.: 71.6 [deg] & 10.0 & 24.0 & 146.5 & 103.9 \\
\hline
\end{tabular}




\begin{tabular}{|c|c|c|c|c|c|c|}
\hline Solution & Vehicle & Maneuver & $\begin{array}{l}\text { Propellant } \\
\text { Consumed by } \\
\text { Vehicle [kg] }\end{array}$ & $\begin{array}{c}\text { Propellant } \\
\text { Consumed by } \\
\text { Constellation } \\
\text { [kg] }\end{array}$ & $\begin{array}{c}\text { Total } \\
\text { Coverage } \\
\text { Time [hr] } \\
\text { Washington, } \\
\text { D.C. }\end{array}$ & $\begin{array}{c}\text { Total } \\
\text { Coverage } \\
\text { Time [hr] } \\
\text { Montevideo }\end{array}$ \\
\hline 49 & 3 & Ecc. Minimizer & 4.1 & 24.0 & 146.5 & 103.9 \\
\hline 50 & 1 & SMA and Ecc. Maximizer - Initial Peri.: 282.7 [deg] & 10.0 & 24.4 & 146.1 & 104.4 \\
\hline 50 & 2 & SMA Maximizer - Initial Peri.: 71.6 [deg] & 10.0 & 24.4 & 146.1 & 104.4 \\
\hline 50 & 3 & Ecc. Minimizer & 4.4 & 24.4 & 146.1 & 104.4 \\
\hline 51 & 1 & SMA Maximizer - Initial Peri.: 51.4 [deg] & 10.0 & 23.9 & 145.7 & 103.3 \\
\hline 51 & 2 & SMA and Ecc. Maximizer - Initial Peri.: 37.2 [deg] & 10.0 & 23.9 & 145.7 & 103.3 \\
\hline 51 & 3 & Ecc. Minimizer & 3.9 & 23.9 & 145.7 & 103.3 \\
\hline 52 & 1 & Incl. Minimizer - Initial RAAN: 209.3 [deg] & 2.4 & 12.9 & 145.4 & 63.4 \\
\hline 52 & 2 & SMA and Ecc. Maximizer - Initial Peri.: 190.6 [deg] & 10.0 & 12.9 & 145.4 & 63.4 \\
\hline 52 & 3 & SMA and Ecc. Maximizer & 0.5 & 12.9 & 145.4 & 63.4 \\
\hline 53 & 1 & SMA and Ecc. Maximizer - Initial Peri.: 357.8 [deg] & 9.9 & 24.5 & 144.9 & 105.2 \\
\hline 53 & 2 & SMA and Ecc. Maximizer - Initial Peri.: 351.2 [deg] & 10.0 & 24.5 & 144.9 & 105.2 \\
\hline 53 & 3 & Ecc. Minimizer & 4.6 & 24.5 & 144.9 & 105.2 \\
\hline 54 & 1 & SMA Maximizer - Initial Peri.: 323.2 [deg] & 0.0 & 10.5 & 144.7 & 66.9 \\
\hline 54 & 2 & SMA and Ecc. Maximizer - Initial Peri.: 143.7 [deg] & 10.0 & 10.5 & 144.7 & 66.9 \\
\hline 54 & 3 & SMA Maximizer & 0.5 & 10.5 & 144.7 & 66.9 \\
\hline 55 & 1 & SMA and Ecc. Maximizer - Initial Peri.: 37.9 [deg] & 10.0 & 25.3 & 144.4 & 105.7 \\
\hline 55 & 2 & SMA Maximizer - Initial Peri.: 332.3 [deg] & 10.0 & 25.3 & 144.4 & 105.7 \\
\hline 55 & 3 & Ecc. Minimizer & 5.3 & 25.3 & 144.4 & 105.7 \\
\hline 56 & 1 & SMA Maximizer - Initial Peri.: 281.0 [deg] & 0.7 & 10.0 & 144.0 & 62.2 \\
\hline 56 & 2 & SMA and Ecc. Maximizer - Initial Peri.: 180.6 [deg] & 8.8 & 10.0 & 144.0 & 62.2 \\
\hline
\end{tabular}




\begin{tabular}{|c|c|c|c|c|c|c|}
\hline Solution & Vehicle & Maneuver & $\begin{array}{c}\text { Propellant } \\
\text { Consumed by } \\
\text { Vehicle [kg] }\end{array}$ & $\begin{array}{c}\text { Propellant } \\
\text { Consumed by } \\
\text { Constellation } \\
\text { [kg] }\end{array}$ & $\begin{array}{c}\text { Total } \\
\text { Coverage } \\
\text { Time [hr] } \\
\text { Washington, } \\
\text { D.C. }\end{array}$ & $\begin{array}{c}\text { Total } \\
\text { Coverage } \\
\text { Time [hr] } \\
\text { Montevideo }\end{array}$ \\
\hline 56 & 3 & SMA and Ecc. Maximizer & 0.5 & 10.0 & 144.0 & 62.2 \\
\hline 57 & 1 & SMA and Ecc. Maximizer - Initial Peri.: 312.9 [deg] & 9.9 & 23.3 & 143.8 & 101.4 \\
\hline 57 & 2 & SMA and Ecc. Maximizer - Initial Peri.: 66.9 [deg] & 9.3 & 23.3 & 143.8 & 101.4 \\
\hline 57 & 3 & Ecc. Minimizer & 4.1 & 23.3 & 143.8 & 101.4 \\
\hline 58 & 1 & Zero maneuver & 0.0 & 10.7 & 143.7 & 66.9 \\
\hline 58 & 2 & SMA Maximizer - Initial Peri.: 251.4 [deg] & 10.0 & 10.7 & 143.7 & 66.9 \\
\hline 58 & 3 & SMA Maximizer w/Constant Ecc. & 0.7 & 10.7 & 143.7 & 66.9 \\
\hline 59 & 1 & SMA and Ecc. Maximizer - Initial Peri.: 312.9 [deg] & 9.9 & 23.1 & 143.4 & 102.0 \\
\hline 59 & 2 & SMA and Ecc. Maximizer - Initial Peri.: 358.3 [deg] & 9.1 & 23.1 & 143.4 & 102.0 \\
\hline 59 & 3 & Ecc. Minimizer & 4.1 & 23.1 & 143.4 & 102.0 \\
\hline 60 & 1 & SMA Maximizer - Initial Peri.: 37.9 [deg] & 10.0 & 24.9 & 143.3 & 107.1 \\
\hline 60 & 2 & SMA Maximizer - Initial Peri.: 22.4 [deg] & 10.0 & 24.9 & 143.3 & 107.1 \\
\hline 60 & 3 & Ecc. Minimizer & 4.9 & 24.9 & 143.3 & 107.1 \\
\hline 61 & 1 & SMA and Ecc. Maximizer - Initial Peri.: 238.8 [deg] & 9.5 & 23.2 & 143.2 & 102.5 \\
\hline 61 & 2 & SMA and Ecc. Maximizer - Initial Peri.: 359.0 [deg] & 9.6 & 23.2 & 143.2 & 102.5 \\
\hline 61 & 3 & Ecc. Minimizer & 4.1 & 23.2 & 143.2 & 102.5 \\
\hline 62 & 1 & SMA Maximizer - Initial Peri.: 41.4 [deg] & 10.0 & 25.3 & 142.8 & 107.5 \\
\hline 62 & 2 & SMA Maximizer - Initial Peri.: 7.1 [deg] & 10.0 & 25.3 & 142.8 & 107.5 \\
\hline 62 & 3 & Ecc. Minimizer & 5.3 & 25.3 & 142.8 & 107.5 \\
\hline 63 & 1 & SMA Maximizer - Initial Peri.: 231.3 [deg] & 1.5 & 12.0 & 142.6 & 76.7 \\
\hline 63 & 2 & SMA and Ecc. Maximizer - Initial Peri.: 354.8 [deg] & 10.0 & 12.0 & 142.6 & 76.7 \\
\hline
\end{tabular}




\begin{tabular}{|c|c|c|c|c|c|c|}
\hline Solution & Vehicle & Maneuver & $\begin{array}{c}\text { Propellant } \\
\text { Consumed by } \\
\text { Vehicle [kg] }\end{array}$ & $\begin{array}{c}\text { Propellant } \\
\text { Consumed by } \\
\text { Constellation } \\
{[\mathrm{kg}]}\end{array}$ & $\begin{array}{c}\text { Total } \\
\text { Coverage } \\
\text { Time [hr] } \\
\text { Washington, } \\
\text { D.C. }\end{array}$ & $\begin{array}{c}\text { Total } \\
\text { Coverage } \\
\text { Time [hr] } \\
\text { Montevideo }\end{array}$ \\
\hline 63 & 3 & SMA and Ecc. Maximizer & 0.5 & 12.0 & 142.6 & 76.7 \\
\hline 64 & 1 & SMA and Ecc. Maximizer - Initial Peri.: 288.5 [deg] & 2.4 & 11.3 & 142.4 & 69.0 \\
\hline 64 & 2 & SMA and Ecc. Maximizer - Initial Peri.: 119.4 [deg] & 8.4 & 11.3 & 142.4 & 69.0 \\
\hline 64 & 3 & SMA Maximizer & 0.5 & 11.3 & 142.4 & 69.0 \\
\hline 65 & 1 & SMA Maximizer - Initial Peri.: 24.3 [deg] & 0.5 & 9.9 & 142.3 & 62.4 \\
\hline 65 & 2 & SMA and Ecc. Maximizer - Initial Peri.: 203.3 [deg] & 8.7 & 9.9 & 142.3 & 62.4 \\
\hline 65 & 3 & SMA Maximizer w/Constant Ecc. & 0.7 & 9.9 & 142.3 & 62.4 \\
\hline 66 & 1 & SMA Maximizer - Initial Peri.: 35.7 [deg] & 9.5 & 22.5 & 142.0 & 101.1 \\
\hline 66 & 2 & SMA and Ecc. Maximizer - Initial Peri.: 37.2 [deg] & 9.1 & 22.5 & 142.0 & 101.1 \\
\hline 66 & 3 & Ecc. Minimizer & 3.9 & 22.5 & 142.0 & 101.1 \\
\hline 67 & 1 & Zero maneuver & 0.0 & 9.4 & 141.3 & 61.1 \\
\hline 67 & 2 & SMA Maximizer - Initial Peri.: 203.3 [deg] & 8.7 & 9.4 & 141.3 & 61.1 \\
\hline 67 & 3 & SMA Maximizer w/Constant Ecc. & 0.7 & 9.4 & 141.3 & 61.1 \\
\hline 68 & 1 & SMA Maximizer - Initial Peri.: 128.5 [deg] & 1.4 & 10.9 & 140.4 & 69.4 \\
\hline 68 & 2 & SMA and Ecc. Maximizer - Initial Peri.: 284.1 [deg] & 9.4 & 10.9 & 140.4 & 69.4 \\
\hline 68 & 3 & SMA Maximizer & 0.0 & 10.9 & 140.4 & 69.4 \\
\hline 69 & 1 & SMA and Ecc. Maximizer - Initial Peri.: 128.5 [deg] & 3.0 & 12.4 & 140.0 & 78.1 \\
\hline 69 & 2 & SMA and Ecc. Maximizer - Initial Peri.: 357.7 [deg] & 9.4 & 12.4 & 140.0 & 78.1 \\
\hline 69 & 3 & Arg.Peri. Maximizer w/Constant Others & 0.0 & 12.4 & 140.0 & 78.1 \\
\hline 70 & 1 & SMA Maximizer - Initial Peri.: 288.5 [deg] & 2.4 & 11.8 & 139.8 & 76.1 \\
\hline 70 & 2 & SMA and Ecc. Maximizer - Initial Peri.: 34.9 [deg] & 8.9 & 11.8 & 139.8 & 76.1 \\
\hline
\end{tabular}




\begin{tabular}{|c|c|c|c|c|c|c|}
\hline Solution & Vehicle & Maneuver & $\begin{array}{l}\text { Propellant } \\
\text { Consumed by } \\
\text { Vehicle [kg] }\end{array}$ & $\begin{array}{c}\text { Propellant } \\
\text { Consumed by } \\
\text { Constellation } \\
\text { [kg] }\end{array}$ & $\begin{array}{c}\text { Total } \\
\text { Coverage } \\
\text { Time [hr] } \\
\text { Washington, } \\
\text { D.C. }\end{array}$ & $\begin{array}{c}\text { Total } \\
\text { Coverage } \\
\text { Time [hr] } \\
\text { Montevideo }\end{array}$ \\
\hline 70 & 3 & SMA and Ecc. Maximizer & 0.5 & 11.8 & 139.8 & 76.1 \\
\hline 71 & 1 & SMA and Ecc. Maximizer - Initial Peri.: 75.1 [deg] & 0.5 & 8.4 & 139.5 & 57.2 \\
\hline 71 & 2 & SMA and Ecc. Maximizer - Initial Peri.: 178.2 [deg] & 7.4 & 8.4 & 139.5 & 57.2 \\
\hline 71 & 3 & SMA and Ecc. Maximizer & 0.5 & 8.4 & 139.5 & 57.2 \\
\hline 72 & 1 & SMA Maximizer w/Constant Ecc. & 0.7 & 9.8 & 139.4 & 67.0 \\
\hline 72 & 2 & SMA and Ecc. Maximizer - Initial Peri.: 303.6 [deg] & 8.7 & 9.8 & 139.4 & 67.0 \\
\hline 72 & 3 & SMA Maximizer & 0.5 & 9.8 & 139.4 & 67.0 \\
\hline 73 & 1 & SMA Maximizer - Initial Peri.: 171.6 [deg] & 0.1 & 10.6 & 139.0 & 73.9 \\
\hline 73 & 2 & SMA Maximizer - Initial Peri.: 5.2 [deg] & 10.0 & 10.6 & 139.0 & 73.9 \\
\hline 73 & 3 & SMA Maximizer & 0.5 & 10.6 & 139.0 & 73.9 \\
\hline 74 & 1 & SMA and Ecc. Maximizer - Initial Peri.: 288.5 [deg] & 2.4 & 11.4 & 138.6 & 74.8 \\
\hline 74 & 2 & SMA and Ecc. Maximizer - Initial Peri.: 34.9 [deg] & 8.5 & 11.4 & 138.6 & 74.8 \\
\hline 74 & 3 & SMA Maximizer & 0.5 & 11.4 & 138.6 & 74.8 \\
\hline 75 & 1 & Zero maneuver & 0.0 & 8.6 & 137.2 & 60.5 \\
\hline 75 & 2 & SMA Maximizer - Initial Peri.: 251.4 [deg] & 7.9 & 8.6 & 137.2 & 60.5 \\
\hline 75 & 3 & SMA Maximizer w/Constant Ecc. & 0.7 & 8.6 & 137.2 & 60.5 \\
\hline 76 & 1 & SMA and Ecc. Maximizer - Initial Peri.: 150.1 [deg] & 0.1 & 10.4 & 136.7 & 73.6 \\
\hline 76 & 2 & SMA and Ecc. Maximizer - Initial Peri.: 17.5 [deg] & 9.6 & 10.4 & 136.7 & 73.6 \\
\hline 76 & 3 & SMA Maximizer w/Constant Ecc. & 0.7 & 10.4 & 136.7 & 73.6 \\
\hline 77 & 1 & SMA and Ecc. Maximizer - Initial Peri.: 352.4 [deg] & 10.0 & 28.4 & 136.3 & 109.4 \\
\hline 77 & 2 & SMA and Ecc. Maximizer - Initial Peri.: 310.2 [deg] & 9.8 & 28.4 & 136.3 & 109.4 \\
\hline
\end{tabular}




\begin{tabular}{|c|c|c|c|c|c|c|}
\hline Solution & Vehicle & Maneuver & $\begin{array}{c}\text { Propellant } \\
\text { Consumed by } \\
\text { Vehicle [kg] }\end{array}$ & $\begin{array}{c}\text { Propellant } \\
\text { Consumed by } \\
\text { Constellation } \\
{[\mathrm{kg}]}\end{array}$ & $\begin{array}{c}\text { Total } \\
\text { Coverage } \\
\text { Time [hr] } \\
\text { Washington, } \\
\text { D.C. }\end{array}$ & $\begin{array}{c}\text { Total } \\
\text { Coverage } \\
\text { Time [hr] } \\
\text { Montevideo }\end{array}$ \\
\hline 77 & 3 & Ecc. Minimizer & 8.6 & 28.4 & 136.3 & 109.4 \\
\hline 78 & 1 & SMA and Ecc. Maximizer - Initial Peri.: 273.5 [deg] & 10.0 & 28.4 & 135.8 & 109.7 \\
\hline 78 & 2 & SMA and Ecc. Maximizer - Initial Peri.: 99.3 [deg] & 9.8 & 28.4 & 135.8 & 109.7 \\
\hline 78 & 3 & Ecc. Minimizer & 8.6 & 28.4 & 135.8 & 109.7 \\
\hline 79 & 1 & Zero maneuver & 0.0 & 8.9 & 135.6 & 65.2 \\
\hline 79 & 2 & SMA and Ecc. Maximizer - Initial Peri.: 307.0 [deg] & 8.2 & 8.9 & 135.6 & 65.2 \\
\hline 79 & 3 & SMA Maximizer w/Constant Ecc. & 0.7 & 8.9 & 135.6 & 65.2 \\
\hline 80 & 1 & SMA Maximizer - Initial Peri.: 104.9 [deg] & 0.7 & 9.6 & 135.5 & 70.1 \\
\hline 80 & 2 & SMA and Ecc. Maximizer - Initial Peri.: 21.8 [deg] & 8.4 & 9.6 & 135.5 & 70.1 \\
\hline 80 & 3 & SMA Maximizer & 0.5 & 9.6 & 135.5 & 70.1 \\
\hline 81 & 1 & Zero maneuver & 0.0 & 7.7 & 134.8 & 57.0 \\
\hline 81 & 2 & SMA Maximizer - Initial Peri.: 182.8 [deg] & 7.7 & 7.7 & 134.8 & 57.0 \\
\hline 81 & 3 & Zero maneuver & 0.0 & 7.7 & 134.8 & 57.0 \\
\hline 82 & 1 & SMA Maximizer - Initial Peri.: 75.1 [deg] & 0.5 & 9.1 & 134.2 & 68.9 \\
\hline 82 & 2 & SMA and Ecc. Maximizer - Initial Peri.: 19.9 [deg] & 8.2 & 9.1 & 134.2 & 68.9 \\
\hline 82 & 3 & SMA Maximizer & 0.5 & 9.1 & 134.2 & 68.9 \\
\hline 83 & 1 & SMA Maximizer - Initial Peri.: 42.8 [deg] & 0.5 & 9.1 & 134.1 & 68.9 \\
\hline 83 & 2 & SMA and Ecc. Maximizer - Initial Peri.: 19.9 [deg] & 8.2 & 9.1 & 134.1 & 68.9 \\
\hline 83 & 3 & SMA Maximizer & 0.5 & 9.1 & 134.1 & 68.9 \\
\hline 84 & 1 & Zero maneuver & 0.0 & 9.6 & 133.4 & 72.2 \\
\hline 84 & 2 & SMA and Ecc. Maximizer - Initial Peri.: 17.5 [deg] & 9.6 & 9.6 & 133.4 & 72.2 \\
\hline
\end{tabular}




\begin{tabular}{|c|c|c|c|c|c|c|}
\hline Solution & Vehicle & Maneuver & $\begin{array}{c}\text { Propellant } \\
\text { Consumed by } \\
\text { Vehicle [kg] }\end{array}$ & $\begin{array}{c}\text { Propellant } \\
\text { Consumed by } \\
\text { Constellation } \\
{[\mathrm{kg}]}\end{array}$ & $\begin{array}{c}\text { Total } \\
\text { Coverage } \\
\text { Time [hr] } \\
\text { Washington, } \\
\text { D.C. }\end{array}$ & $\begin{array}{c}\text { Total } \\
\text { Coverage } \\
\text { Time [hr] } \\
\text { Montevideo }\end{array}$ \\
\hline 84 & 3 & SMA Maximizer w/Constant Ecc. & 0.0 & 9.6 & 133.4 & 72.2 \\
\hline 85 & 1 & SMA Maximizer w/Constant Ecc. & 9.9 & 27.9 & 132.8 & 110.9 \\
\hline 85 & 2 & SMA and Ecc. Maximizer - Initial Peri.: 46.3 [deg] & 9.7 & 27.9 & 132.8 & 110.9 \\
\hline 85 & 3 & Ecc. Minimizer & 8.2 & 27.9 & 132.8 & 110.9 \\
\hline 86 & 1 & Zero maneuver & 0.0 & 8.4 & 132.8 & 66.7 \\
\hline 86 & 2 & SMA Maximizer - Initial Peri.: 7.1 [deg] & 7.9 & 8.4 & 132.8 & 66.7 \\
\hline 86 & 3 & SMA Maximizer & 0.5 & 8.4 & 132.8 & 66.7 \\
\hline 87 & 1 & Zero maneuver & 0.0 & 8.2 & 132.5 & 63.5 \\
\hline 87 & 2 & SMA Maximizer - Initial Peri.: 315.9 [deg] & 7.4 & 8.2 & 132.5 & 63.5 \\
\hline 87 & 3 & SMA Maximizer w/Constant Ecc. & 0.7 & 8.2 & 132.5 & 63.5 \\
\hline 88 & 1 & SMA Maximizer w/Constant Ecc. & 0.3 & 8.1 & 132.4 & 64.5 \\
\hline 88 & 2 & SMA and Ecc. Maximizer - Initial Peri.: 50.7 [deg] & 7.3 & 8.1 & 132.4 & 64.5 \\
\hline 88 & 3 & SMA and Ecc. Maximizer & 0.5 & 8.1 & 132.4 & 64.5 \\
\hline 89 & 1 & SMA Maximizer - Initial Peri.: 104.9 [deg] & 0.2 & 9.3 & 132.3 & 71.0 \\
\hline 89 & 2 & SMA Maximizer - Initial Peri.: 7.1 [deg] & 9.1 & 9.3 & 132.3 & 71.0 \\
\hline 89 & 3 & SMA Maximizer w/Constant Ecc. & 0.0 & 9.3 & 132.3 & 71.0 \\
\hline 90 & 1 & Zero maneuver & 0.0 & 9.1 & 132.3 & 69.9 \\
\hline 90 & 2 & SMA and Ecc. Maximizer - Initial Peri.: 49.6 [deg] & 9.0 & 9.1 & 132.3 & 69.9 \\
\hline 90 & 3 & Arg.Peri. Maximizer w/Constant Others & 0.1 & 9.1 & 132.3 & 69.9 \\
\hline 91 & 1 & SMA Maximizer - Initial Peri.: 6.4 [deg] & 0.7 & 7.3 & 132.3 & 57.5 \\
\hline 91 & 2 & SMA Maximizer - Initial Peri.: 281.4 [deg] & 6.1 & 7.3 & 132.3 & 57.5 \\
\hline
\end{tabular}




\begin{tabular}{|c|c|c|c|c|c|c|}
\hline Solution & Vehicle & Maneuver & $\begin{array}{c}\text { Propellant } \\
\text { Consumed by } \\
\text { Vehicle [kg] }\end{array}$ & $\begin{array}{c}\text { Propellant } \\
\text { Consumed by } \\
\text { Constellation } \\
{[\mathrm{kg}]}\end{array}$ & $\begin{array}{c}\text { Total } \\
\text { Coverage } \\
\text { Time [hr] } \\
\text { Washington, } \\
\text { D.C. }\end{array}$ & $\begin{array}{c}\text { Total } \\
\text { Coverage } \\
\text { Time [hr] } \\
\text { Montevideo }\end{array}$ \\
\hline 91 & 3 & SMA and Ecc. Maximizer & 0.5 & 7.3 & 132.3 & 57.5 \\
\hline 92 & 1 & SMA and Ecc. Maximizer - Initial Peri.: 71.0 [deg] & 10.0 & 29.0 & 131.8 & 111.9 \\
\hline 92 & 2 & SMA and Ecc. Maximizer - Initial Peri.: 77.2 [deg] & 9.6 & 29.0 & 131.8 & 111.9 \\
\hline 92 & 3 & Ecc. Minimizer & 9.4 & 29.0 & 131.8 & 111.9 \\
\hline 93 & 1 & SMA and Ecc. Maximizer - Initial Peri.: 71.0 [deg] & 10.0 & 28.8 & 131.5 & 113.6 \\
\hline 93 & 2 & SMA and Ecc. Maximizer - Initial Peri.: 0.3 [deg] & 9.6 & 28.8 & 131.5 & 113.6 \\
\hline 93 & 3 & Ecc. Minimizer & 9.2 & 28.8 & 131.5 & 113.6 \\
\hline 94 & 1 & SMA Maximizer - Initial Peri.: 37.9 [deg] & 0.7 & 8.3 & 130.8 & 65.0 \\
\hline 94 & 2 & SMA and Ecc. Maximizer - Initial Peri.: 333.1 [deg] & 7.4 & 8.3 & 130.8 & 65.0 \\
\hline 94 & 3 & SMA and Ecc. Maximizer & 0.2 & 8.3 & 130.8 & 65.0 \\
\hline 95 & 1 & SMA and Ecc. Maximizer - Initial Peri.: 234.2 [deg] & 10.0 & 29.7 & 130.7 & 115.0 \\
\hline 95 & 2 & SMA and Ecc. Maximizer - Initial Peri.: 350.7 [deg] & 10.0 & 29.7 & 130.7 & 115.0 \\
\hline 95 & 3 & Ecc. Minimizer & 9.7 & 29.7 & 130.7 & 115.0 \\
\hline 96 & 1 & SMA Maximizer - Initial Peri.: 41.4 [deg] & 10.0 & 29.9 & 130.4 & 115.9 \\
\hline 96 & 2 & SMA and Ecc. Maximizer - Initial Peri.: 7.1 [deg] & 10.0 & 29.9 & 130.4 & 115.9 \\
\hline 96 & 3 & Ecc. Minimizer & 9.9 & 29.9 & 130.4 & 115.9 \\
\hline 97 & 1 & SMA Maximizer w/Constant Ecc. & 10.0 & 27.5 & 130.2 & 108.4 \\
\hline 97 & 2 & SMA and Ecc. Maximizer - Initial Peri.: 351.2 [deg] & 8.9 & 27.5 & 130.2 & 108.4 \\
\hline 97 & 3 & Ecc. Minimizer & 8.6 & 27.5 & 130.2 & 108.4 \\
\hline 98 & 1 & SMA Maximizer - Initial Peri.: 313.1 [deg] & 0.3 & 6.6 & 129.6 & 56.6 \\
\hline 98 & 2 & SMA Maximizer - Initial Peri.: 300.9 [deg] & 5.8 & 6.6 & 129.6 & 56.6 \\
\hline
\end{tabular}




\begin{tabular}{|c|c|c|c|c|c|c|}
\hline Solution & Vehicle & Maneuver & $\begin{array}{c}\text { Propellant } \\
\text { Consumed by } \\
\text { Vehicle [kg] }\end{array}$ & $\begin{array}{c}\text { Propellant } \\
\text { Consumed by } \\
\text { Constellation } \\
{[\mathrm{kg}]}\end{array}$ & $\begin{array}{c}\text { Total } \\
\text { Coverage } \\
\text { Time [hr] } \\
\text { Washington, } \\
\text { D.C. }\end{array}$ & $\begin{array}{c}\text { Total } \\
\text { Coverage } \\
\text { Time [hr] } \\
\text { Montevideo }\end{array}$ \\
\hline 98 & 3 & SMA and Ecc. Maximizer & 0.5 & 6.6 & 129.6 & 56.6 \\
\hline 99 & 1 & SMA and Ecc. Maximizer - Initial Peri.: 158.6 [deg] & 3.4 & 6.6 & 129.5 & 49.8 \\
\hline 99 & 2 & SMA Maximizer - Initial Peri.: 105.2 [deg] & 2.6 & 6.6 & 129.5 & 49.8 \\
\hline 99 & 3 & SMA and Ecc. Maximizer & 0.5 & 6.6 & 129.5 & 49.8 \\
\hline 100 & 1 & SMA Maximizer - Initial Peri.: 234.2 [deg] & 10.0 & 28.8 & 129.4 & 111.7 \\
\hline 100 & 2 & SMA and Ecc. Maximizer - Initial Peri.: 51.2 [deg] & 9.3 & 28.8 & 129.4 & 111.7 \\
\hline 100 & 3 & Ecc. Minimizer & 9.4 & 28.8 & 129.4 & 111.7 \\
\hline 101 & 1 & Zero maneuver & 0.0 & 8.2 & 129.3 & 67.3 \\
\hline 101 & 2 & SMA and Ecc. Maximizer - Initial Peri.: 7.1 [deg] & 8.2 & 8.2 & 129.3 & 67.3 \\
\hline 101 & 3 & SMA and Ecc. Maximizer & 0.0 & 8.2 & 129.3 & 67.3 \\
\hline 102 & 1 & SMA and Ecc. Maximizer - Initial Peri.: 221.3 [deg] & 10.0 & 27.0 & 129.3 & 107.6 \\
\hline 102 & 2 & SMA and Ecc. Maximizer - Initial Peri.: 351.2 [deg] & 8.5 & 27.0 & 129.3 & 107.6 \\
\hline 102 & 3 & Ecc. Minimizer & 8.6 & 27.0 & 129.3 & 107.6 \\
\hline 103 & 1 & SMA Maximizer - Initial Peri.: 42.8 [deg] & 9.7 & 28.0 & 128.7 & 111.1 \\
\hline 103 & 2 & SMA and Ecc. Maximizer - Initial Peri.: 37.2 [deg] & 9.1 & 28.0 & 128.7 & 111.1 \\
\hline 103 & 3 & Ecc. Minimizer & 9.1 & 28.0 & 128.7 & 111.1 \\
\hline 104 & 1 & SMA Maximizer - Initial Peri.: 340.9 [deg] & 10.0 & 29.4 & 128.6 & 113.9 \\
\hline 104 & 2 & SMA and Ecc. Maximizer - Initial Peri.: 359.0 [deg] & 9.4 & 29.4 & 128.6 & 113.9 \\
\hline 104 & 3 & Ecc. Minimizer & 10.0 & 29.4 & 128.6 & 113.9 \\
\hline 105 & 1 & SMA Maximizer - Initial Peri.: 35.7 [deg] & 0.3 & 6.8 & 128.2 & 57.6 \\
\hline 105 & 2 & SMA and Ecc. Maximizer - Initial Peri.: 300.9 [deg] & 5.8 & 6.8 & 128.2 & 57.6 \\
\hline
\end{tabular}




\begin{tabular}{|c|c|c|c|c|c|c|}
\hline Solution & Vehicle & Maneuver & $\begin{array}{c}\text { Propellant } \\
\text { Consumed by } \\
\text { Vehicle [kg] }\end{array}$ & $\begin{array}{c}\text { Propellant } \\
\text { Consumed by } \\
\text { Constellation } \\
{[\mathrm{kg}]}\end{array}$ & $\begin{array}{c}\text { Total } \\
\text { Coverage } \\
\text { Time [hr] } \\
\text { Washington, } \\
\text { D.C. }\end{array}$ & $\begin{array}{c}\text { Total } \\
\text { Coverage } \\
\text { Time [hr] } \\
\text { Montevideo }\end{array}$ \\
\hline 105 & 3 & SMA Maximizer w/Constant Ecc. & 0.7 & 6.8 & 128.2 & 57.6 \\
\hline 106 & 1 & SMA Maximizer - Initial Peri.: 61.1 [deg] & 0.4 & 6.8 & 127.9 & 59.5 \\
\hline 106 & 2 & SMA and Ecc. Maximizer - Initial Peri.: 350.1 [deg] & 5.9 & 6.8 & 127.9 & 59.5 \\
\hline 106 & 3 & SMA Maximizer & 0.5 & 6.8 & 127.9 & 59.5 \\
\hline 107 & 1 & Zero maneuver & 0.0 & 7.4 & 127.4 & 64.3 \\
\hline 107 & 2 & SMA and Ecc. Maximizer - Initial Peri.: 349.0 [deg] & 7.4 & 7.4 & 127.4 & 64.3 \\
\hline 107 & 3 & SMA and Ecc. Maximizer & 0.0 & 7.4 & 127.4 & 64.3 \\
\hline 108 & 1 & Zero maneuver & 0.0 & 5.9 & 127.2 & 53.7 \\
\hline 108 & 2 & SMA and Ecc. Maximizer - Initial Peri.: 122.7 [deg] & 5.9 & 5.9 & 127.2 & 53.7 \\
\hline 108 & 3 & SMA and Ecc. Maximizer & 0.0 & 5.9 & 127.2 & 53.7 \\
\hline 109 & 1 & SMA Maximizer - Initial Peri.: 239.4 [deg] & 0.0 & 6.2 & 127.1 & 55.4 \\
\hline 109 & 2 & SMA Maximizer w/Constant Ecc. & 6.2 & 6.2 & 127.1 & 55.4 \\
\hline 109 & 3 & Zero maneuver & 0.0 & 6.2 & 127.1 & 55.4 \\
\hline 110 & 1 & SMA and Ecc. Maximizer - Initial Peri.: 108.2 [deg] & 0.7 & 5.3 & 126.9 & 47.7 \\
\hline 110 & 2 & SMA and Ecc. Maximizer - Initial Peri.: 194.2 [deg] & 3.9 & 5.3 & 126.9 & 47.7 \\
\hline 110 & 3 & SMA Maximizer w/Constant Ecc. & 0.7 & 5.3 & 126.9 & 47.7 \\
\hline 111 & 1 & SMA Maximizer - Initial Peri.: 106.4 [deg] & 9.9 & 27.7 & 126.8 & 109.7 \\
\hline 111 & 2 & SMA and Ecc. Maximizer - Initial Peri.: 3.7 [deg] & 8.5 & 27.7 & 126.8 & 109.7 \\
\hline 111 & 3 & Ecc. Minimizer & 9.3 & 27.7 & 126.8 & 109.7 \\
\hline 112 & 1 & SMA Maximizer - Initial Peri.: 253.5 [deg] & 0.5 & 5.7 & 125.9 & 53.6 \\
\hline 112 & 2 & SMA and Ecc. Maximizer - Initial Peri.: 72.8 [deg] & 4.9 & 5.7 & 125.9 & 53.6 \\
\hline
\end{tabular}




\begin{tabular}{|c|c|c|c|c|c|c|}
\hline Solution & Vehicle & Maneuver & $\begin{array}{c}\text { Propellant } \\
\text { Consumed by } \\
\text { Vehicle [kg] }\end{array}$ & $\begin{array}{c}\text { Propellant } \\
\text { Consumed by } \\
\text { Constellation } \\
\text { [kg] }\end{array}$ & $\begin{array}{c}\text { Total } \\
\text { Coverage } \\
\text { Time [hr] } \\
\text { Washington, } \\
\text { D.C. }\end{array}$ & $\begin{array}{c}\text { Total } \\
\text { Coverage } \\
\text { Time [hr] } \\
\text { Montevideo }\end{array}$ \\
\hline 112 & 3 & SMA and Ecc. Maximizer & 0.4 & 5.7 & 125.9 & 53.6 \\
\hline 113 & 1 & SMA Maximizer - Initial Peri.: 336.1 [deg] & 0.1 & 5.9 & 124.9 & 56.1 \\
\hline 113 & 2 & SMA and Ecc. Maximizer - Initial Peri.: 300.9 [deg] & 5.8 & 5.9 & 124.9 & 56.1 \\
\hline 113 & 3 & Zero maneuver & 0.0 & 5.9 & 124.9 & 56.1 \\
\hline 114 & 1 & SMA Maximizer - Initial Peri.: 281.0 [deg] & 0.3 & 5.0 & 124.4 & 50.1 \\
\hline 114 & 2 & SMA and Ecc. Maximizer - Initial Peri.: 86.3 [deg] & 4.3 & 5.0 & 124.4 & 50.1 \\
\hline 114 & 3 & SMA Maximizer & 0.5 & 5.0 & 124.4 & 50.1 \\
\hline 115 & 1 & SMA Maximizer - Initial Peri.: 104.9 [deg] & 0.7 & 4.3 & 124.1 & 45.8 \\
\hline 115 & 2 & SMA and Ecc. Maximizer - Initial Peri.: 260.5 [deg] & 3.1 & 4.3 & 124.1 & 45.8 \\
\hline 115 & 3 & SMA and Ecc. Maximizer & 0.5 & 4.3 & 124.1 & 45.8 \\
\hline 116 & 1 & Zero maneuver & 0.0 & 6.1 & 123.7 & 59.3 \\
\hline 116 & 2 & SMA Maximizer - Initial Peri.: 37.2 [deg] & 6.1 & 6.1 & 123.7 & 59.3 \\
\hline 116 & 3 & Arg.Peri. Maximizer w/Constant Others & 0.0 & 6.1 & 123.7 & 59.3 \\
\hline 117 & 1 & SMA and Ecc. Maximizer - Initial Peri.: 96.8 [deg] & 0.7 & 4.6 & 123.6 & 46.6 \\
\hline 117 & 2 & SMA and Ecc. Maximizer - Initial Peri.: 194.2 [deg] & 3.9 & 4.6 & 123.6 & 46.6 \\
\hline 117 & 3 & Ecc. Maximizer & 0.0 & 4.6 & 123.6 & 46.6 \\
\hline 118 & 1 & SMA and Ecc. Maximizer - Initial Peri.: 303.6 [deg] & 0.5 & 4.8 & 123.0 & 48.1 \\
\hline 118 & 2 & SMA and Ecc. Maximizer - Initial Peri.: 130.7 [deg] & 4.3 & 4.8 & 123.0 & 48.1 \\
\hline 118 & 3 & Zero maneuver & 0.0 & 4.8 & 123.0 & 48.1 \\
\hline 119 & 1 & SMA Maximizer - Initial Peri.: 203.3 [deg] & 0.9 & 5.4 & 122.8 & 51.9 \\
\hline 119 & 2 & SMA and Ecc. Maximizer - Initial Peri.: 70.5 [deg] & 4.4 & 5.4 & 122.8 & 51.9 \\
\hline
\end{tabular}




\begin{tabular}{|c|c|c|c|c|c|c|}
\hline Solution & Vehicle & Maneuver & $\begin{array}{c}\text { Propellant } \\
\text { Consumed by } \\
\text { Vehicle [kg] }\end{array}$ & $\begin{array}{c}\text { Propellant } \\
\text { Consumed by } \\
\text { Constellation } \\
{[\mathrm{kg}]}\end{array}$ & $\begin{array}{c}\text { Total } \\
\text { Coverage } \\
\text { Time [hr] } \\
\text { Washington, } \\
\text { D.C. }\end{array}$ & $\begin{array}{c}\text { Total } \\
\text { Coverage } \\
\text { Time [hr] } \\
\text { Montevideo }\end{array}$ \\
\hline 119 & 3 & SMA and Ecc. Maximizer & 0.0 & 5.4 & 122.8 & 51.9 \\
\hline 120 & 1 & Zero maneuver & 0.0 & 3.9 & 122.2 & 45.1 \\
\hline 120 & 2 & SMA and Ecc. Maximizer - Initial Peri.: 194.2 [deg] & 3.9 & 3.9 & 122.2 & 45.1 \\
\hline 120 & 3 & Ecc. Maximizer & 0.0 & 3.9 & 122.2 & 45.1 \\
\hline 121 & 1 & SMA Maximizer - Initial Peri.: 251.7 [deg] & 0.5 & 4.1 & 121.7 & 47.5 \\
\hline 121 & 2 & SMA and Ecc. Maximizer - Initial Peri.: 22.4 [deg] & 3.1 & 4.1 & 121.7 & 47.5 \\
\hline 121 & 3 & SMA Maximizer & 0.5 & 4.1 & 121.7 & 47.5 \\
\hline 122 & 1 & SMA and Ecc. Maximizer - Initial Peri.: 183.6 [deg] & 0.2 & 3.8 & 121.3 & 46.9 \\
\hline 122 & 2 & SMA and Ecc. Maximizer - Initial Peri.: 25.0 [deg] & 3.1 & 3.8 & 121.3 & 46.9 \\
\hline 122 & 3 & SMA and Ecc. Maximizer & 0.5 & 3.8 & 121.3 & 46.9 \\
\hline 123 & 1 & SMA Maximizer - Initial Peri.: 247.4 [deg] & 1.7 & 5.7 & 120.4 & 53.2 \\
\hline 123 & 2 & SMA and Ecc. Maximizer - Initial Peri.: 7.1 [deg] & 3.7 & 5.7 & 120.4 & 53.2 \\
\hline 123 & 3 & Ecc. Minimizer & 0.3 & 5.7 & 120.4 & 53.2 \\
\hline 124 & 1 & SMA and Ecc. Maximizer - Initial Peri.: 96.8 [deg] & 0.7 & 4.4 & 120.3 & 48.9 \\
\hline 124 & 2 & SMA and Ecc. Maximizer - Initial Peri.: 71.6 [deg] & 3.7 & 4.4 & 120.3 & 48.9 \\
\hline 124 & 3 & Zero maneuver & 0.0 & 4.4 & 120.3 & 48.9 \\
\hline 125 & 1 & SMA and Ecc. Maximizer - Initial Peri.: 187.2 [deg] & 0.7 & 4.5 & 119.6 & 51.4 \\
\hline 125 & 2 & SMA and Ecc. Maximizer - Initial Peri.: 350.1 [deg] & 3.9 & 4.5 & 119.6 & 51.4 \\
\hline 125 & 3 & SMA and Ecc. Maximizer & 0.0 & 4.5 & 119.6 & 51.4 \\
\hline 126 & 1 & SMA and Ecc. Maximizer - Initial Peri.: 104.9 [deg] & 0.7 & 4.5 & 119.5 & 51.5 \\
\hline 126 & 2 & SMA and Ecc. Maximizer - Initial Peri.: 350.1 [deg] & 3.9 & 4.5 & 119.5 & 51.5 \\
\hline
\end{tabular}




\begin{tabular}{|c|c|c|c|c|c|c|}
\hline Solution & Vehicle & Maneuver & $\begin{array}{c}\text { Propellant } \\
\text { Consumed by } \\
\text { Vehicle [kg] }\end{array}$ & $\begin{array}{c}\text { Propellant } \\
\text { Consumed by } \\
\text { Constellation } \\
{[\mathrm{kg}]}\end{array}$ & $\begin{array}{c}\text { Total } \\
\text { Coverage } \\
\text { Time [hr] } \\
\text { Washington, } \\
\text { D.C. }\end{array}$ & $\begin{array}{c}\text { Total } \\
\text { Coverage } \\
\text { Time [hr] } \\
\text { Montevideo }\end{array}$ \\
\hline 126 & 3 & SMA Maximizer & 0.0 & 4.5 & 119.5 & 51.5 \\
\hline 127 & 1 & SMA Maximizer - Initial Peri.: 75.1 [deg] & 0.2 & 2.8 & 118.7 & 42.8 \\
\hline 127 & 2 & SMA and Ecc. Maximizer - Initial Peri.: 19.9 [deg] & 2.1 & 2.8 & 118.7 & 42.8 \\
\hline 127 & 3 & SMA and Ecc. Maximizer & 0.5 & 2.8 & 118.7 & 42.8 \\
\hline 128 & 1 & SMA Maximizer - Initial Peri.: 352.4 [deg] & 0.5 & 3.1 & 117.8 & 43.5 \\
\hline 128 & 2 & SMA Maximizer - Initial Peri.: 105.2 [deg] & 2.6 & 3.1 & 117.8 & 43.5 \\
\hline 128 & 3 & Zero maneuver & 0.0 & 3.1 & 117.8 & 43.5 \\
\hline 129 & 1 & SMA and Ecc. Maximizer - Initial Peri.: 68.0 [deg] & 0.7 & 3.3 & 117.5 & 44.3 \\
\hline 129 & 2 & SMA and Ecc. Maximizer - Initial Peri.: 357.7 [deg] & 1.9 & 3.3 & 117.5 & 44.3 \\
\hline 129 & 3 & SMA Maximizer w/Constant Ecc. & 0.7 & 3.3 & 117.5 & 44.3 \\
\hline 130 & 1 & SMA and Ecc. Maximizer - Initial Peri.: 128.5 [deg] & 3.0 & 3.6 & 116.9 & 44.3 \\
\hline 130 & 2 & SMA and Ecc. Maximizer - Initial Peri.: 357.7 [deg] & 0.6 & 3.6 & 116.9 & 44.3 \\
\hline 130 & 3 & Arg.Peri. Maximizer w/Constant Others & 0.0 & 3.6 & 116.9 & 44.3 \\
\hline 131 & 1 & SMA Maximizer - Initial Peri.: 119.3 [deg] & 0.8 & 1.9 & 116.7 & 38.8 \\
\hline 131 & 2 & SMA and Ecc. Maximizer - Initial Peri.: 149.5 [deg] & 0.6 & 1.9 & 116.7 & 38.8 \\
\hline 131 & 3 & SMA and Ecc. Maximizer & 0.5 & 1.9 & 116.7 & 38.8 \\
\hline 132 & 1 & SMA and Ecc. Maximizer - Initial Peri.: 128.5 [deg] & 1.4 & 2.2 & 116.4 & 39.4 \\
\hline 132 & 2 & SMA and Ecc. Maximizer - Initial Peri.: 308.7 [deg] & 0.2 & 2.2 & 116.4 & 39.4 \\
\hline 132 & 3 & SMA and Ecc. Maximizer & 0.5 & 2.2 & 116.4 & 39.4 \\
\hline 133 & 1 & SMA Maximizer - Initial Peri.: 128.5 [deg] & 1.4 & 2.2 & 115.4 & 40.1 \\
\hline 133 & 2 & SMA and Ecc. Maximizer - Initial Peri.: 70.5 [deg] & 0.5 & 2.2 & 115.4 & 40.1 \\
\hline
\end{tabular}




\begin{tabular}{|c|c|c|c|c|c|c|}
\hline Solution & Vehicle & Maneuver & $\begin{array}{c}\text { Propellant } \\
\text { Consumed by } \\
\text { Vehicle [kg] }\end{array}$ & $\begin{array}{c}\text { Propellant } \\
\text { Consumed by } \\
\text { Constellation } \\
{[\mathrm{kg}]}\end{array}$ & $\begin{array}{c}\text { Total } \\
\text { Coverage } \\
\text { Time [hr] } \\
\text { Washington, } \\
\text { D.C. }\end{array}$ & $\begin{array}{c}\text { Total } \\
\text { Coverage } \\
\text { Time [hr] } \\
\text { Montevideo }\end{array}$ \\
\hline 133 & 3 & SMA and Ecc. Maximizer & 0.3 & 2.2 & 115.4 & 40.1 \\
\hline 134 & 1 & SMA Maximizer - Initial Peri.: 37.9 [deg] & 2.3 & 2.8 & 114.8 & 42.4 \\
\hline 134 & 2 & SMA and Ecc. Maximizer - Initial Peri.: 333.1 [deg] & 0.2 & 2.8 & 114.8 & 42.4 \\
\hline 134 & 3 & SMA and Ecc. Maximizer & 0.2 & 2.8 & 114.8 & 42.4 \\
\hline 135 & 1 & Zero maneuver & 0.0 & 1.0 & 114.0 & 37.1 \\
\hline 135 & 2 & SMA and Ecc. Maximizer - Initial Peri.: 255.4 [deg] & 0.5 & 1.0 & 114.0 & 37.1 \\
\hline 135 & 3 & SMA and Ecc. Maximizer & 0.5 & 1.0 & 114.0 & 37.1 \\
\hline 136 & 1 & SMA and Ecc. Maximizer - Initial Peri.: 187.2 [deg] & 0.5 & 1.3 & 111.5 & 38.8 \\
\hline 136 & 2 & SMA Maximizer - Initial Peri.: 7.1 [deg] & 0.7 & 1.3 & 111.5 & 38.8 \\
\hline 136 & 3 & Zero maneuver & 0.0 & 1.3 & 111.5 & 38.8 \\
\hline 137 & 1 & SMA Maximizer - Initial Peri.: 281.0 [deg] & 0.9 & 1.3 & 111.4 & 39.3 \\
\hline 137 & 2 & SMA and Ecc. Maximizer - Initial Peri.: 86.7 [deg] & 0.4 & 1.3 & 111.4 & 39.3 \\
\hline 137 & 3 & SMA Maximizer & 0.0 & 1.3 & 111.4 & 39.3 \\
\hline 138 & 1 & SMA Maximizer - Initial Peri.: 275.1 [deg] & 1.5 & 1.5 & 110.4 & 39.5 \\
\hline 138 & 2 & Zero maneuver & 0.0 & 1.5 & 110.4 & 39.5 \\
\hline 138 & 3 & SMA Maximizer & 0.0 & 1.5 & 110.4 & 39.5 \\
\hline 139 & 1 & SMA and Ecc. Maximizer - Initial Peri.: 39.1 [deg] & 1.0 & 1.0 & 109.8 & 38.1 \\
\hline 139 & 2 & Zero maneuver & 0.0 & 1.0 & 109.8 & 38.1 \\
\hline 139 & 3 & Zero maneuver & 0.0 & 1.0 & 109.8 & 38.1 \\
\hline 140 & 1 & Zero maneuver & 0.0 & 0.2 & 108.8 & 36.5 \\
\hline 140 & 2 & SMA and Ecc. Maximizer - Initial Peri.: 349.0 [deg] & 0.2 & 0.2 & 108.8 & 36.5 \\
\hline
\end{tabular}




\begin{tabular}{|l|l|l|l|l|l|l|}
\hline Solution & Vehicle & & Maneuver & $\begin{array}{c}\text { Propellant } \\
\text { Consumed by } \\
\text { Vehicle [kg] }\end{array}$ & $\begin{array}{c}\text { Propellant } \\
\text { Consumed by } \\
\text { Constellation } \\
{[\mathrm{kg}]}\end{array}$ & $\begin{array}{c}\text { Total } \\
\text { Coverage } \\
\text { Time [hr] } \\
\text { Washington, } \\
\text { D.C. }\end{array}$ \\
$\begin{array}{c}\text { Total } \\
\text { Coverage } \\
\text { Time [hr] } \\
\text { Montevideo }\end{array}$ \\
\hline 140 & 3 & SMA and Ecc. Maximizer & 0.0 & 0.2 & 108.8 & 36.5 \\
\hline 141 & 1 & Zero maneuver & 0.0 & 0.0 & 108.0 \\
\hline 141 & 2 & Zero maneuver & 0.0 & 0.0 & 108.0 & 36.0 \\
\hline
\end{tabular}





\section{Appendix D: Numerical Study of Single-Satellite Coverage Estimation}

\section{Overview}

This section compares and contrasts the numerical coverage obtained using the estimation approach, described in Chapter 3: Estimating Satellite Coverage, versus the explicit approach, described in Chapter 2: Calculating Coverage. The following coverage characteristics are compared over each sidereal day: maximum duration of a coverage window, number of coverage windows, and the total duration of coverage.

For this comparison, some additional details must be discussed in order to account for a discretization error inherent in both approaches. Both approaches determine whether a satellite is situated within a target's LOS cone at discrete, time intervals. The implementations used in this work use constant, time intervals; $\Delta t_{\text {estimation }}$ is used for the estimation approach, and $\Delta t_{\text {explicit }}$ is used for the explicit approach. A satellite moving into an LOS cone to begin a coverage window may not be detected for up to one time interval. A satellite moving out of an LOS cone to end a coverage window may not be detected for up to one time interval. Therefore, the duration of coverage windows may be underreported by up to two time intervals. This discretization error is accounted for in this study in the various measurements listed below.

\section{Maximum Coverage Window Duration [s] (Explicit)}

This value represents the duration of the longest coverage window calculated using the approach described in Chapter 2: Calculating Coverage. To this value is added $2 \Delta t_{\text {explicit }}$ in order to account for the discretization error (assuming that at least one, explicit, coverage window has been detected.)

\section{Maximum Coverage Window Duration [s] (Estimated)}

This value represents the duration of the longest coverage window calculated using the approach described in Chapter 3: Estimating Satellite Coverage. To this value is added $2 \Delta t_{\text {estimation }}$ in order to account for the discretization error (assuming that at least one, estimated, coverage window has been detected.)

\section{Number of Windows L.B. (Estimated)}

This value represents the lower bound on the number of coverage windows estimated using the approach described in Chapter 3: Estimating Satellite Coverage.

\section{Number of Windows (Explicit)}

This value represents the number of coverage windows calculated using the approach described in Chapter 2: Calculating Coverage. 


\section{Number of Windows L.B. (Estimated)}

This value represents the upper bound on the number of coverage windows estimated using the approach described in Chapter 3: Estimating Satellite Coverage.

\section{Total Coverage Duration L.B. [s] (Estimated)}

This value represents the combined duration of all the coverage windows estimated using the approach described in Chapter 3: Estimating Satellite Coverage.

\section{Total Coverage Duration L.B. [s] (Explicit)}

This value represents the combined duration of all the coverage windows calculated using the approach described in Chapter 2: Calculating Coverage.

\section{Total Coverage Duration U.B. [s] (Explicit)}

This value represents the combined duration of all the coverage windows calculated using the approach described in Chapter 2: Calculating Coverage. To this value is added $2 \Delta t_{\text {explicit }}$ for each coverage window in order to account for the discretization error.

\section{Total Coverage Duration U.B. [s] (Estimated)}

This value represents the combined duration of all the coverage windows estimated using the approach described in Chapter 3: Estimating Satellite Coverage. To this value is added $2 \Delta t_{\text {estimation }}$ multiplied by the estimated, upperbound on the number of coverage windows in order to account for the discretization error.

\section{Parameters of the Explicit Approach}

The frequency of coverage checking, $\Delta t_{\text {explicit }}$, is 30 seconds. Visibility windows briefer than 60 seconds are filtered out.

\section{Parameters of the Estimation Approach}

The orbit-fixed sphere uses a discretization grid-spacing of 1-degree arclength. The inertial sphere's latitude is discretized into 5-minute slots. The frequency of coverage checking, $\Delta t_{\text {estimation }}$, is 5-minutes.

\section{Example}

The orbit used in this example is described by Table 21. Figure 64 display the time-evolution of the visibility map over 120 days, and Table 22 lists the daily coverage of the target over 120 sidereal days. The figure clearly shows the strong effect that the rotation of the apsides (see Section 3.5.2: Multiple-Day, Single-Satellite Coverage Estimation) has on the orientation of the orbit, and hence, its visibility map. The largest swath of the orbit's coverage footprint lies across Washington, D.C.'s latitude at the initial date. By the $80^{\text {th }}$ sidereal day, the coverage footprint is absent across the latitude. By the $120^{\text {th }}$ sidereal day, 
the coverage footprint encroaches Washington, D.C.'s latitude once again. Table 22 also reflects this trend in coverage. Additionally, explicit coverage always falls within the estimated coverage bounds.

For comparison with a wider range of targets, the coverage of multiple targets over the first sidereal day was also investigated. Table 23 compares the coverage obtained across the Washington, D.C.'s longitude. Table 24 compares the coverage obtained across Washington, D.C.'s latitude. In both cases, explicit coverage always falls within the estimated coverage bounds. 
Table 21: Scenario properties used for the coverage estimation study

\begin{tabular}{|c|c|c|c|c|c|c|c|c|c|c|c|c|c|}
\hline \multicolumn{14}{|c|}{ General } \\
\hline \multicolumn{7}{|c|}{ Initial Date } & \multicolumn{7}{|c|}{ 2006-01-01 00:00:00 UTC } \\
\hline \multicolumn{7}{|c|}{ Final Date } & \multicolumn{7}{|c|}{ 2006-04-30 16:08:10 UTC (i.e. 120 sidereal days later) } \\
\hline \multicolumn{7}{|c|}{ Frequency of Coverage Checking } & \multicolumn{7}{|c|}{$30[\mathrm{~s}]$} \\
\hline \multicolumn{7}{|c|}{ Minimum Elevation Angle } & \multicolumn{7}{|l|}{20 [deg] } \\
\hline \multicolumn{7}{|c|}{ Gravity Mode } & \multicolumn{7}{|c|}{ 3x3 Spherical Harmonic EGM-96 } \\
\hline \multicolumn{14}{|c|}{ Targets } \\
\hline \multicolumn{4}{|c|}{ Name } & \multicolumn{5}{|c|}{ Latitude [deg] } & \multicolumn{5}{|c|}{ Longitude [deg] } \\
\hline \multicolumn{4}{|c|}{ Washington, D.C., USA } & \multicolumn{3}{|c|}{$38^{\circ} 53^{\prime} \mathrm{N}$} & & & \multicolumn{5}{|c|}{$77^{\circ} 2^{\prime} \mathrm{W}$} \\
\hline \multicolumn{14}{|c|}{ Satellite } \\
\hline Name & $\begin{array}{c}\text { Initial } \\
\text { Total } \\
\text { Mass [kg] }\end{array}$ & $\begin{array}{c}\text { Initial } \\
\text { Propellant } \\
\text { Mass [kg] }\end{array}$ & $\begin{array}{c}\text { Specific } \\
\text { Velocity } \\
{[\mathrm{m} / \mathrm{s}]}\end{array}$ & $\begin{array}{l}\text { Maximum } \\
\text { Thrust [N] }\end{array}$ & $\begin{array}{l}\text { Initia } \\
\text { Posi }\end{array}$ & $\begin{array}{l}\mathrm{ECI} X- \\
\text { on }[\mathrm{m}]\end{array}$ & $\begin{array}{l}\text { Initial ECI Y- } \\
\text { Position [m] }\end{array}$ & $\begin{array}{l}\text { Initial ECI Z } \\
\text { Position [m] }\end{array}$ & $\begin{array}{l}Z- \\
\text { ] }\end{array}$ & $\begin{array}{l}\text { nitial ECI X- } \\
\text { Speed }[\mathrm{m} / \mathrm{s}]\end{array}$ & $\begin{array}{l}\text { Initial E } \\
\text { Speed }\end{array}$ & $\begin{array}{l}\mathrm{CI} I \mathrm{Y} \\
{[\mathrm{m} / \mathrm{s}]}\end{array}$ & $\begin{array}{l}\text { Initial ECI Z- } \\
\text { Speed }[\mathrm{m} / \mathrm{s}]\end{array}$ \\
\hline Sat.1 & 100 & N/A & N/A & N/A & $-5,79$ & 809.5 & 0.0 & $-3,348,521.6$ & & 0.0 & $-9,258.3$ & & 0.0 \\
\hline \multicolumn{2}{|c|}{ Name } & \multicolumn{2}{|c|}{ SMA [ER] } & \multicolumn{2}{|c|}{ Eccentricity } & \multicolumn{2}{|c|}{ Inclination [deg] } & \multicolumn{2}{|c|}{ RAAN [deg] } & \multicolumn{2}{|c|}{$\begin{array}{l}\text { Arg. Periapsis } \\
\text { [deg] }\end{array}$} & \multicolumn{2}{|c|}{$\begin{array}{l}\text { True Anomaly } \\
\text { [deg] }\end{array}$} \\
\hline \multicolumn{2}{|l|}{ Sat. 1} & \multicolumn{2}{|l|}{1.875} & \multicolumn{2}{|l|}{0.444} & \multicolumn{2}{|l|}{30.0} & \multicolumn{2}{|c|}{270.0} & \multicolumn{2}{|l|}{270.0} & \multicolumn{2}{|l|}{0.0} \\
\hline
\end{tabular}



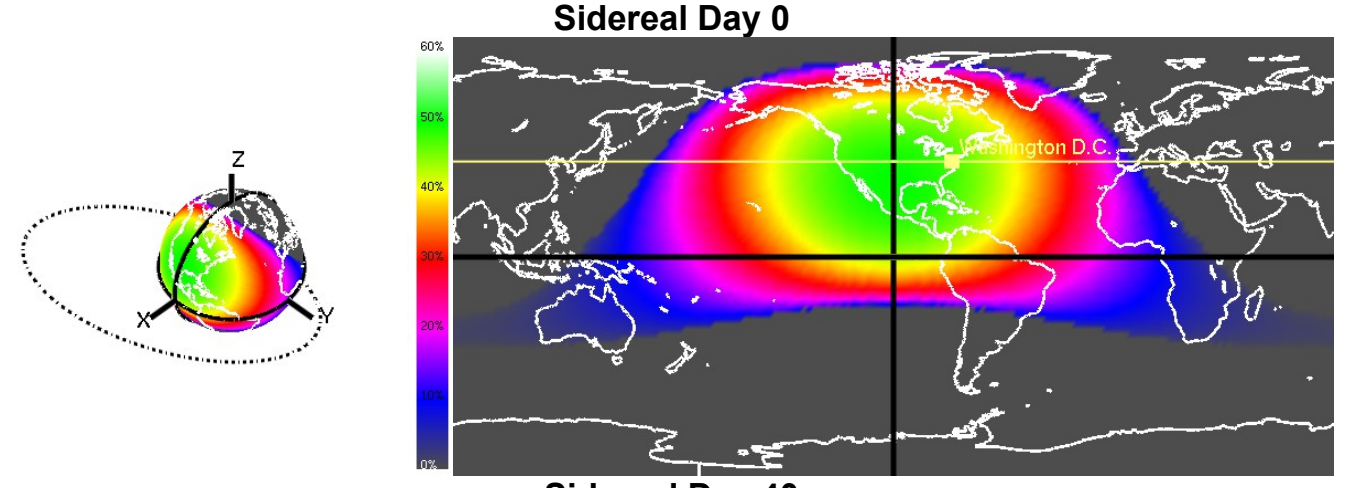

\section{Sidereal Day 40}
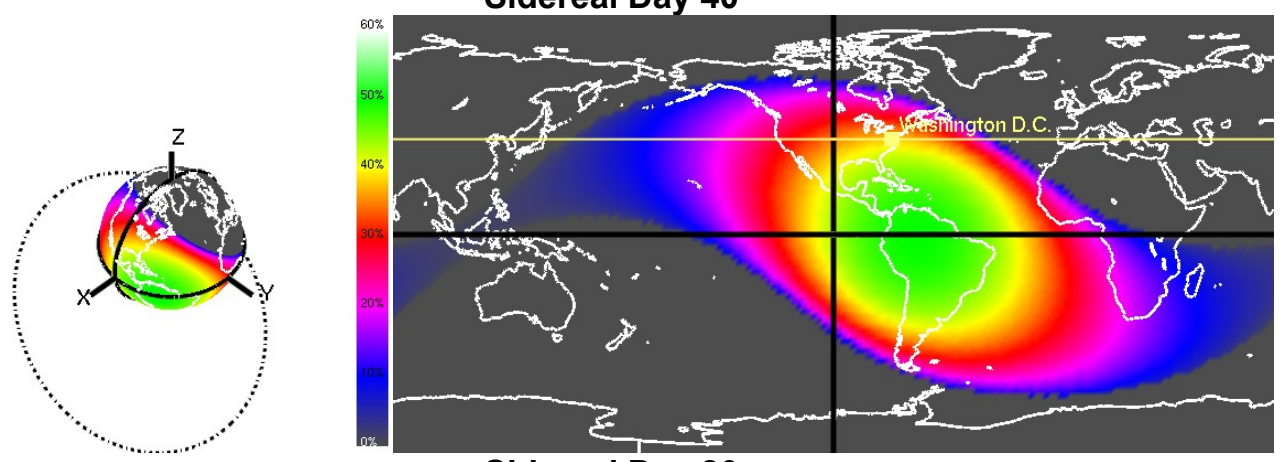

\section{Sidereal Day 80}

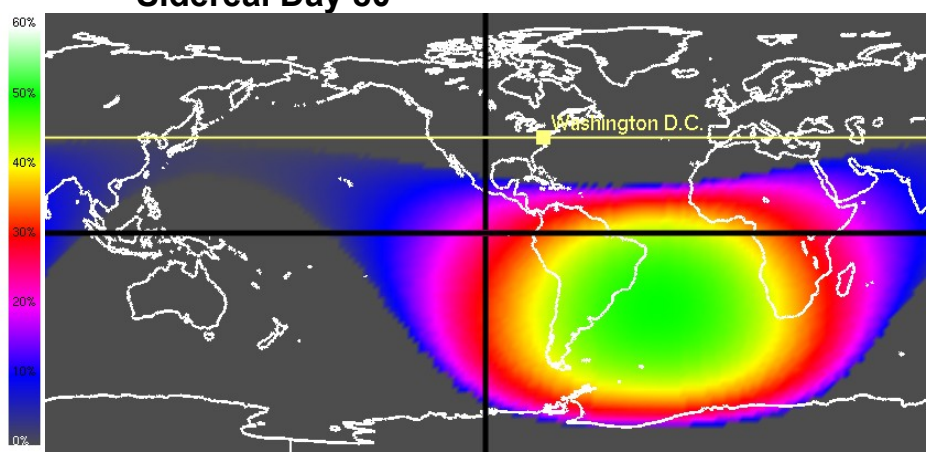

Sidereal Day 120

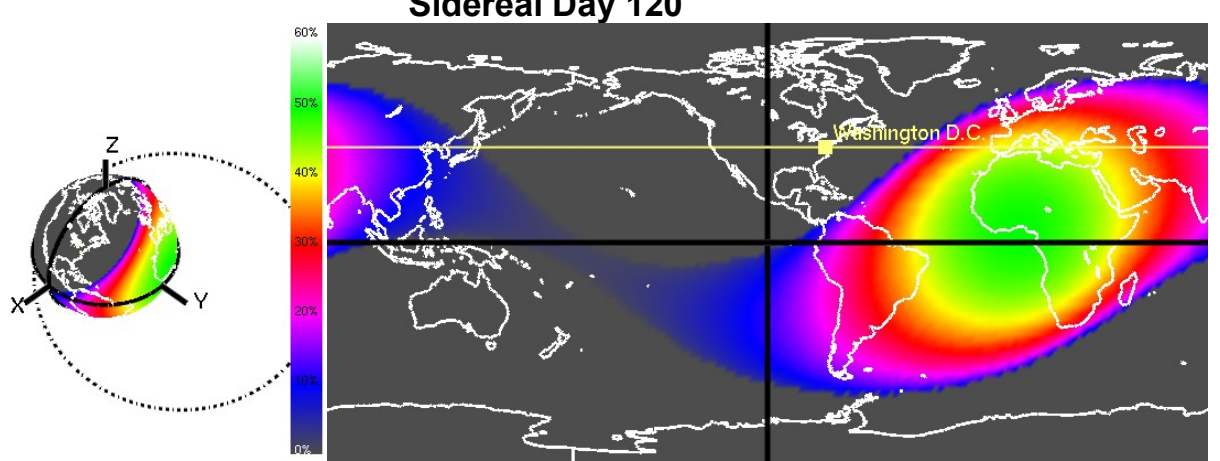

Figure 64: Shown is the 120-day evolution of the visibility map for the sample orbit used in the numerical study. Washington, D.C. and its latitude are marked in yellow. The continental outlines are marked in white. Areas in gray are not visible from the satellite orbit. Areas in any other color are visible/covered from a satellite orbit. 
Table 22: Comparison of explicit coverage versus estimated coverage across Washington, D.C.'s longitude over 120 sidereal days

\begin{tabular}{|c|c|c|c|c|c|c|c|c|c|}
\hline $\begin{array}{c}\text { Sidereal } \\
\text { Day }\end{array}$ & $\begin{array}{c}\text { Maximum } \\
\text { Coverage } \\
\text { Window } \\
\text { Duration [s] } \\
\text { (Explicit) }\end{array}$ & $\begin{array}{l}\text { Maximum } \\
\text { Coverage } \\
\text { Window } \\
\text { Duration [s] } \\
\text { (Estimated) }\end{array}$ & $\begin{array}{c}\text { Number of } \\
\text { Windows } \\
\text { L.B. } \\
\text { (Estimated) }\end{array}$ & $\begin{array}{l}\text { Number of } \\
\text { Windows } \\
\text { (Explicit) }\end{array}$ & $\begin{array}{c}\text { Number of } \\
\text { Windows } \\
\text { L.B. } \\
\text { (Estimated) }\end{array}$ & $\begin{array}{c}\text { Total } \\
\text { Coverage } \\
\text { Duration } \\
\text { L.B. [s] } \\
\text { (Estimated) }\end{array}$ & $\begin{array}{c}\text { Total } \\
\text { Coverage } \\
\text { Duration } \\
\text { L.B. [s] } \\
\text { (Explicit) }\end{array}$ & $\begin{array}{c}\text { Total } \\
\text { Coverage } \\
\text { Duration } \\
\text { U.B. [s] } \\
\text { (Explicit) }\end{array}$ & $\begin{array}{c}\text { Total } \\
\text { Coverage } \\
\text { Duration } \\
\text { U.B. [s] } \\
\text { (Estimated) }\end{array}$ \\
\hline 1 & 6480 & 8020 & 3 & 3 & 5 & 12820 & 16744 & 16924 & 22671 \\
\hline 2 & 7230 & 8020 & 3 & 4 & 5 & 12520 & 16466 & 16706 & 22671 \\
\hline 3 & 7470 & 8020 & 3 & 3 & 5 & 13120 & 19350 & 19530 & 22420 \\
\hline 4 & 6630 & 8020 & 3 & 3 & 5 & 13420 & 14806 & 14986 & 22200 \\
\hline 5 & 7290 & 7800 & 3 & 4 & 5 & 13120 & 17684 & 17924 & 22120 \\
\hline 6 & 6984 & 7800 & 3 & 3 & 5 & 13500 & 18864 & 19044 & 22119 \\
\hline 7 & 6780 & 8020 & 3 & 5 & 5 & 13420 & 15124 & 15424 & 22372 \\
\hline 8 & 7320 & 8019 & 3 & 3 & 5 & 13119 & 17281 & 17461 & 22119 \\
\hline 9 & 6120 & 8019 & 3 & 3 & 5 & 13419 & 17077 & 17257 & 22119 \\
\hline 10 & 6900 & 7800 & 3 & 4 & 5 & 12900 & 15533 & 15773 & 21518 \\
\hline 11 & 7320 & 7800 & 3 & 3 & 5 & 13118 & 18660 & 18840 & 21518 \\
\hline 12 & 6060 & 7800 & 3 & 4 & 5 & 13417 & 15679 & 15919 & 21817 \\
\hline 13 & 6990 & 7800 & 3 & 4 & 5 & 13118 & 16181 & 16421 & 21600 \\
\hline 14 & 7317 & 8017 & 3 & 3 & 5 & 12856 & 18657 & 18837 & 21517 \\
\hline 15 & 6210 & 8017 & 3 & 5 & 6 & 12555 & 14464 & 14764 & 22117 \\
\hline 16 & 7020 & 7716 & 3 & 3 & 5 & 12256 & 15719 & 15899 & 21216 \\
\hline 17 & 6360 & 7717 & 3 & 3 & 5 & 11917 & 16869 & 17049 & 20917 \\
\hline 18 & 6330 & 7716 & 3 & 4 & 5 & 11700 & 13940 & 14180 & 21216 \\
\hline 19 & 7020 & 7717 & 3 & 3 & 5 & 11275 & 17490 & 17670 & 20917 \\
\hline 20 & 5850 & 7500 & 3 & 4 & 5 & 10973 & 15322 & 15562 & 20616 \\
\hline
\end{tabular}




\begin{tabular}{|c|c|c|c|c|c|c|c|c|c|}
\hline $\begin{array}{c}\text { Sidereal } \\
\text { Day }\end{array}$ & $\begin{array}{c}\text { Maximum } \\
\text { Coverage } \\
\text { Window } \\
\text { Duration [s] } \\
\text { (Explicit) }\end{array}$ & \begin{tabular}{|} 
Maximum \\
Coverage \\
Window \\
Duration [s] \\
(Estimated)
\end{tabular} & $\begin{array}{c}\text { Number of } \\
\text { Windows } \\
\text { L.B. } \\
\text { (Estimated) }\end{array}$ & $\begin{array}{l}\text { Number of } \\
\text { Windows } \\
\text { (Explicit) }\end{array}$ & $\begin{array}{c}\text { Number of } \\
\text { Windows } \\
\text { L.B. } \\
\text { (Estimated) }\end{array}$ & $\begin{array}{c}\text { Total } \\
\text { Coverage } \\
\text { Duration } \\
\text { L.B. [s] } \\
\text { (Estimated) }\end{array}$ & $\begin{array}{c}\text { Total } \\
\text { Coverage } \\
\text { Duration } \\
\text { L.B. [s] } \\
\text { (Explicit) }\end{array}$ & $\begin{array}{c}\text { Total } \\
\text { Coverage } \\
\text { Duration } \\
\text { U.B. [s] } \\
\text { (Explicit) }\end{array}$ & $\begin{array}{c}\text { Total } \\
\text { Coverage } \\
\text { Duration } \\
\text { U.B. [s] } \\
\text { (Estimated) }\end{array}$ \\
\hline 21 & 6450 & 7500 & 3 & 4 & 5 & 10800 & 13148 & 13388 & 20316 \\
\hline 22 & 6960 & 7500 & 3 & 3 & 5 & 10759 & 17370 & 17550 & 20316 \\
\hline 23 & 5460 & 7716 & 3 & 4 & 5 & 10459 & 13654 & 13894 & 20316 \\
\hline 24 & 6480 & 7416 & 3 & 3 & 5 & 9900 & 13766 & 13946 & 20016 \\
\hline 25 & 6240 & 7416 & 3 & 3 & 5 & 9861 & 16122 & 16302 & 20016 \\
\hline 26 & 5580 & 7416 & 3 & 5 & 5 & 9000 & 11824 & 12124 & 19716 \\
\hline 27 & 6480 & 7415 & 3 & 3 & 5 & 8915 & 15524 & 15704 & 19415 \\
\hline 28 & 5580 & 7116 & 3 & 4 & 5 & 8616 & 14334 & 14574 & 19116 \\
\hline 29 & 5670 & 7115 & 3 & 3 & 5 & 8015 & 9845 & 10025 & 18815 \\
\hline 30 & 6420 & 7115 & 3 & 3 & 5 & 7500 & 15600 & 15780 & 18815 \\
\hline 31 & 4530 & 6900 & 3 & 4 & 5 & 6900 & 12037 & 12277 & 18215 \\
\hline 32 & 5730 & 6815 & 3 & 3 & 5 & 6515 & 11633 & 11813 & 18215 \\
\hline 33 & 6135 & 6815 & 3 & 3 & 5 & 5915 & 14955 & 15135 & 18215 \\
\hline 34 & 4650 & 6600 & 3 & 5 & 5 & 5700 & 7534 & 7834 & 17915 \\
\hline 35 & 5700 & 6600 & 3 & 3 & 4 & 5615 & 13181 & 13361 & 17100 \\
\hline 36 & 4800 & 6515 & 3 & 4 & 4 & 5100 & 12657 & 12897 & 16715 \\
\hline 37 & 4740 & 6300 & 2 & 3 & 4 & 4800 & 7533 & 7713 & 16500 \\
\hline 38 & 5640 & 6514 & 2 & 3 & 4 & 5014 & 13320 & 13500 & 16414 \\
\hline 39 & 3540 & 6215 & 2 & 4 & 4 & 4715 & 9369 & 9609 & 16115 \\
\hline 40 & 4740 & 6214 & 2 & 3 & 4 & 4500 & 9110 & 9290 & 15514 \\
\hline 41 & 5400 & 6215 & 2 & 3 & 4 & 4415 & 12420 & 12600 & 15515 \\
\hline
\end{tabular}




\begin{tabular}{|c|c|c|c|c|c|c|c|c|c|}
\hline $\begin{array}{c}\text { Sidereal } \\
\text { Day }\end{array}$ & $\begin{array}{c}\text { Maximum } \\
\text { Coverage } \\
\text { Window } \\
\text { Duration [s] } \\
\text { (Explicit) }\end{array}$ & \begin{tabular}{|} 
Maximum \\
Coverage \\
Window \\
Duration [s] \\
(Estimated)
\end{tabular} & $\begin{array}{c}\text { Number of } \\
\text { Windows } \\
\text { L.B. } \\
\text { (Estimated) }\end{array}$ & $\begin{array}{l}\text { Number of } \\
\text { Windows } \\
\text { (Explicit) }\end{array}$ & $\begin{array}{c}\text { Number of } \\
\text { Windows } \\
\text { L.B. } \\
\text { (Estimated) }\end{array}$ & $\begin{array}{c}\text { Total } \\
\text { Coverage } \\
\text { Duration } \\
\text { L.B. [s] } \\
\text { (Estimated) }\end{array}$ & $\begin{array}{c}\text { Total } \\
\text { Coverage } \\
\text { Duration } \\
\text { L.B. [s] } \\
\text { (Explicit) }\end{array}$ & $\begin{array}{c}\text { Total } \\
\text { Coverage } \\
\text { Duration } \\
\text { U.B. [s] } \\
\text { (Explicit) }\end{array}$ & $\begin{array}{c}\text { Total } \\
\text { Coverage } \\
\text { Duration } \\
\text { U.B. [s] } \\
\text { (Estimated) }\end{array}$ \\
\hline 42 & 3630 & 5914 & 2 & 3 & 4 & 4200 & 5092 & 5272 & 14914 \\
\hline 43 & 4680 & 5914 & 2 & 3 & 4 & 4200 & 10478 & 10658 & 14614 \\
\hline 44 & 4560 & 5614 & 2 & 4 & 4 & 3900 & 10200 & 10440 & 14014 \\
\hline 45 & 3660 & 5614 & 2 & 3 & 4 & 3600 & 4980 & 5160 & 14014 \\
\hline 46 & 4500 & 5315 & 2 & 3 & 4 & 3300 & 10440 & 10620 & 13415 \\
\hline 47 & 2772 & 5314 & 2 & 3 & 5 & 3300 & 5622 & 5802 & 14014 \\
\hline 48 & 3600 & 5315 & 2 & 3 & 4 & 3000 & 6318 & 6498 & 13115 \\
\hline 49 & 4200 & 5015 & 2 & 3 & 4 & 3000 & 8820 & 9000 & 12215 \\
\hline 50 & 2670 & 4714 & 2 & 3 & 4 & 2914 & 3954 & 4134 & 11914 \\
\hline 51 & 3480 & 4715 & 2 & 3 & 4 & 2700 & 7415 & 7595 & 11315 \\
\hline 52 & 3780 & 4415 & 2 & 2 & 4 & 2315 & 5460 & 5580 & 10715 \\
\hline 53 & 2610 & 4414 & 2 & 2 & 4 & 2100 & 3150 & 3270 & 10714 \\
\hline 54 & 3240 & 4116 & 2 & 3 & 4 & 2100 & 6720 & 6900 & 10116 \\
\hline 55 & 2775 & 4115 & 2 & 3 & 4 & 2100 & 4695 & 4875 & 9815 \\
\hline 56 & 2520 & 3600 & 2 & 3 & 4 & 2100 & 3375 & 3555 & 9206 \\
\hline 57 & 2820 & 3515 & 2 & 2 & 4 & 2100 & 3900 & 4020 & 8907 \\
\hline 58 & 1800 & 3300 & 2 & 3 & 4 & 1800 & 2607 & 2787 & 8095 \\
\hline 59 & 2280 & 3000 & 2 & 3 & 5 & 1500 & 3963 & 4143 & 7497 \\
\hline 60 & 2100 & 3000 & 2 & 2 & 3 & 1200 & 3180 & 3300 & 5100 \\
\hline 61 & 1680 & 2700 & 2 & 2 & 3 & 1200 & 1980 & 2100 & 4500 \\
\hline 62 & 1860 & 2400 & 1 & 2 & 3 & 900 & 2520 & 2640 & 4500 \\
\hline
\end{tabular}




\begin{tabular}{|c|c|c|c|c|c|c|c|c|c|}
\hline $\begin{array}{c}\text { Sidereal } \\
\text { Day }\end{array}$ & $\begin{array}{c}\text { Maximum } \\
\text { Coverage } \\
\text { Window } \\
\text { Duration [s] } \\
\text { (Explicit) }\end{array}$ & \begin{tabular}{|} 
Maximum \\
Coverage \\
Window \\
Duration [s] \\
(Estimated)
\end{tabular} & $\begin{array}{c}\text { Number of } \\
\text { Windows } \\
\text { L.B. } \\
\text { (Estimated) }\end{array}$ & $\begin{array}{l}\text { Number of } \\
\text { Windows } \\
\text { (Explicit) }\end{array}$ & $\begin{array}{c}\text { Number of } \\
\text { Windows } \\
\text { L.B. } \\
\text { (Estimated) }\end{array}$ & $\begin{array}{c}\text { Total } \\
\text { Coverage } \\
\text { Duration } \\
\text { L.B. [s] } \\
\text { (Estimated) }\end{array}$ & $\begin{array}{c}\text { Total } \\
\text { Coverage } \\
\text { Duration } \\
\text { L.B. [s] } \\
\text { (Explicit) }\end{array}$ & $\begin{array}{c}\text { Total } \\
\text { Coverage } \\
\text { Duration } \\
\text { U.B. [s] } \\
\text { (Explicit) }\end{array}$ & $\begin{array}{c}\text { Total } \\
\text { Coverage } \\
\text { Duration } \\
\text { U.B. [s] } \\
\text { (Estimated) }\end{array}$ \\
\hline 63 & 1170 & 2400 & 1 & 2 & 2 & 600 & 1170 & 1290 & 3600 \\
\hline 64 & 1470 & 2100 & 1 & 2 & 2 & 900 & 1770 & 1890 & 3225 \\
\hline 65 & 1050 & 2100 & 1 & 2 & 2 & 600 & 1650 & 1770 & 3228 \\
\hline 66 & 1020 & 1800 & 1 & 2 & 2 & 531 & 1080 & 1200 & 2700 \\
\hline 67 & 990 & 1737 & 1 & 2 & 2 & 300 & 1290 & 1410 & 2400 \\
\hline 68 & 660 & 1500 & 1 & 1 & 2 & 0 & 600 & 660 & 2400 \\
\hline 69 & 750 & 1448 & 1 & 2 & 3 & 0 & 840 & 960 & 2648 \\
\hline 70 & 390 & 1200 & 1 & 1 & 2 & 0 & 330 & 390 & 1800 \\
\hline 71 & 480 & 1159 & 0 & 1 & 3 & 0 & 420 & 480 & 2359 \\
\hline 72 & 150 & 900 & 0 & 1 & 2 & 0 & 90 & 150 & 1500 \\
\hline 73 & 210 & 894 & 0 & 1 & 5 & 0 & 150 & 210 & 3577 \\
\hline 74 & 0 & 898 & 0 & 0 & 3 & 0 & 0 & 0 & 2098 \\
\hline 75 & 0 & 0 & 0 & 0 & 0 & 0 & 0 & 0 & 0 \\
\hline 76 & 0 & 0 & 0 & 0 & 0 & 0 & 0 & 0 & 0 \\
\hline 77 & 0 & 0 & 0 & 0 & 0 & 0 & 0 & 0 & 0 \\
\hline 78 & 0 & 0 & 0 & 0 & 0 & 0 & 0 & 0 & 0 \\
\hline 79 & 0 & 0 & 0 & 0 & 0 & 0 & 0 & 0 & 0 \\
\hline 80 & 0 & 0 & 0 & 0 & 0 & 0 & 0 & 0 & 0 \\
\hline 81 & 0 & 0 & 0 & 0 & 0 & 0 & 0 & 0 & 0 \\
\hline 82 & 180 & 600 & 0 & 1 & 1 & 0 & 120 & 180 & 600 \\
\hline 83 & 210 & 898 & 0 & 1 & 5 & 0 & 150 & 210 & 3893 \\
\hline
\end{tabular}




\begin{tabular}{|c|c|c|c|c|c|c|c|c|c|}
\hline $\begin{array}{c}\text { Sidereal } \\
\text { Day }\end{array}$ & $\begin{array}{l}\text { Maximum } \\
\text { Coverage } \\
\text { Window } \\
\text { Duration [s] } \\
\text { (Explicit) }\end{array}$ & \begin{tabular}{|} 
Maximum \\
Coverage \\
Window \\
Duration [s] \\
(Estimated)
\end{tabular} & $\begin{array}{c}\text { Number of } \\
\text { Windows } \\
\text { L.B. } \\
\text { (Estimated) }\end{array}$ & $\begin{array}{l}\text { Number of } \\
\text { Windows } \\
\text { (Explicit) }\end{array}$ & $\begin{array}{c}\text { Number of } \\
\text { Windows } \\
\text { L.B. } \\
\text { (Estimated) }\end{array}$ & $\begin{array}{c}\text { Total } \\
\text { Coverage } \\
\text { Duration } \\
\text { L.B. [s] } \\
\text { (Estimated) }\end{array}$ & $\begin{array}{c}\text { Total } \\
\text { Coverage } \\
\text { Duration } \\
\text { L.B. [s] } \\
\text { (Explicit) }\end{array}$ & $\begin{array}{c}\text { Total } \\
\text { Coverage } \\
\text { Duration } \\
\text { U.B. [s] } \\
\text { (Explicit) }\end{array}$ & $\begin{array}{c}\text { Total } \\
\text { Coverage } \\
\text { Duration } \\
\text { U.B. [s] } \\
\text { (Estimated) }\end{array}$ \\
\hline 84 & 420 & 900 & 0 & 1 & 3 & 0 & 360 & 420 & 2397 \\
\hline 85 & 300 & 900 & 0 & 2 & 1 & 0 & 450 & 570 & 900 \\
\hline 86 & 690 & 1200 & 0 & 1 & 3 & 0 & 630 & 690 & 2400 \\
\hline 87 & 540 & 1200 & 1 & 1 & 3 & 0 & 480 & 540 & 2400 \\
\hline 88 & 1020 & 1200 & 1 & 2 & 3 & 0 & 1200 & 1320 & 2400 \\
\hline 89 & 840 & 1500 & 1 & 1 & 3 & 0 & 780 & 840 & 3000 \\
\hline 90 & 1320 & 1500 & 1 & 2 & 2 & 300 & 1740 & 1860 & 2400 \\
\hline 91 & 1260 & 1800 & 1 & 2 & 2 & 300 & 1410 & 1530 & 2700 \\
\hline 92 & 1440 & 2100 & 1 & 2 & 2 & 600 & 2220 & 2340 & 3000 \\
\hline 93 & 1770 & 2100 & 1 & 2 & 2 & 600 & 2190 & 2310 & 3300 \\
\hline 94 & 1380 & 2400 & 1 & 3 & 2 & 900 & 2250 & 2430 & 3600 \\
\hline 95 & 2220 & 2400 & 1 & 2 & 4 & 900 & 3000 & 3120 & 5100 \\
\hline 96 & 1980 & 2700 & 2 & 2 & 3 & 1200 & 2400 & 2520 & 5100 \\
\hline 97 & 2460 & 3000 & 2 & 2 & 4 & 1500 & 3780 & 3900 & 5700 \\
\hline 98 & 2640 & 3300 & 2 & 2 & 4 & 1800 & 3420 & 3540 & 6000 \\
\hline 99 & 2100 & 3300 & 2 & 3 & 3 & 2100 & 4350 & 4530 & 6000 \\
\hline 100 & 3180 & 3600 & 2 & 2 & 3 & 2400 & 4470 & 4590 & 6000 \\
\hline 101 & 2880 & 3600 & 2 & 2 & 4 & 2400 & 3600 & 3720 & 6900 \\
\hline 102 & 3390 & 3900 & 2 & 3 & 3 & 3000 & 5730 & 5910 & 6900 \\
\hline 103 & 3630 & 4200 & 2 & 2 & 3 & 3300 & 4890 & 5010 & 7200 \\
\hline 104 & 3000 & 4200 & 2 & 3 & 3 & 3600 & 6300 & 6480 & 7800 \\
\hline
\end{tabular}




\begin{tabular}{|c|c|c|c|c|c|c|c|c|c|}
\hline $\begin{array}{c}\text { Sidereal } \\
\text { Day }\end{array}$ & $\begin{array}{l}\text { Maximum } \\
\text { Coverage } \\
\text { Window } \\
\text { Duration [s] } \\
\text { (Explicit) }\end{array}$ & $\begin{array}{c}\text { Maximum } \\
\text { Coverage } \\
\text { Window } \\
\text { Duration [s] } \\
\text { (Estimated) }\end{array}$ & $\begin{array}{c}\text { Number of } \\
\text { Windows } \\
\text { L.B. } \\
\text { (Estimated) }\end{array}$ & $\begin{array}{l}\text { Number of } \\
\text { Windows } \\
\text { (Explicit) }\end{array}$ & $\begin{array}{c}\text { Number of } \\
\text { Windows } \\
\text { L.B. } \\
\text { (Estimated) }\end{array}$ & $\begin{array}{c}\text { Total } \\
\text { Coverage } \\
\text { Duration } \\
\text { L.B. [s] } \\
\text { (Estimated) }\end{array}$ & $\begin{array}{c}\text { Total } \\
\text { Coverage } \\
\text { Duration } \\
\text { L.B. [s] } \\
\text { (Explicit) }\end{array}$ & $\begin{array}{c}\text { Total } \\
\text { Coverage } \\
\text { Duration } \\
\text { U.B. [s] } \\
\text { (Explicit) }\end{array}$ & $\begin{array}{c}\text { Total } \\
\text { Coverage } \\
\text { Duration } \\
\text { U.B. [s] } \\
\text { (Estimated) }\end{array}$ \\
\hline 105 & 4110 & 4500 & 2 & 3 & 4 & 3900 & 6240 & 6420 & 8700 \\
\hline 106 & 3900 & 4500 & 2 & 2 & 3 & 3900 & 5100 & 5220 & 8400 \\
\hline 107 & 4110 & 4800 & 2 & 3 & 3 & 4500 & 7680 & 7860 & 8700 \\
\hline 108 & 4560 & 5100 & 2 & 2 & 4 & 4500 & 6480 & 6600 & 9900 \\
\hline 109 & 4050 & 5100 & 2 & 3 & 4 & 4800 & 8130 & 8310 & 10200 \\
\hline 110 & 4860 & 5100 & 2 & 3 & 3 & 5100 & 8280 & 8460 & 9900 \\
\hline 111 & 4890 & 5400 & 2 & 2 & 3 & 5400 & 6720 & 6840 & 10500 \\
\hline 112 & 4590 & 5700 & 2 & 3 & 3 & 6000 & 9630 & 9810 & 10500 \\
\hline 113 & 5340 & 5700 & 2 & 3 & 3 & 6300 & 8490 & 8670 & 11100 \\
\hline 114 & 5070 & 6000 & 2 & 3 & 3 & 6600 & 9720 & 9900 & 11400 \\
\hline 115 & 5430 & 6000 & 2 & 3 & 3 & 7200 & 10320 & 10500 & 11700 \\
\hline 116 & 5700 & 6000 & 2 & 2 & 3 & 7200 & 8400 & 8520 & 12000 \\
\hline 117 & 5160 & 6300 & 2 & 3 & 3 & 7200 & 11460 & 11640 & 12600 \\
\hline 118 & 5940 & 6300 & 2 & 3 & 3 & 7500 & 10560 & 10740 & 12900 \\
\hline 119 & 5910 & 6300 & 2 & 3 & 3 & 7500 & 10590 & 10770 & 13200 \\
\hline 120 & 5670 & 6600 & 2 & 3 & 4 & 8100 & 12180 & 12360 & 14100 \\
\hline
\end{tabular}


Table 23: Comparison of explicit coverage versus estimated coverage across Washington, D.C.'s longitude over the first sidereal day

\begin{tabular}{|c|c|c|c|c|c|c|c|c|c|}
\hline Target & $\begin{array}{l}\text { Maximum } \\
\text { Coverage } \\
\text { Window } \\
\text { Duration [s] } \\
\text { (Explicit) }\end{array}$ & $\begin{array}{c}\text { Maximum } \\
\text { Coverage } \\
\text { Window } \\
\text { Duration [s] } \\
\text { (Estimated) }\end{array}$ & $\begin{array}{c}\text { Number of } \\
\text { Windows } \\
\text { L.B. } \\
\text { (Estimated) }\end{array}$ & $\begin{array}{l}\text { Number of } \\
\text { Windows } \\
\text { (Explicit) }\end{array}$ & $\begin{array}{c}\text { Number of } \\
\text { Windows } \\
\text { L.B. } \\
\text { (Estimated) }\end{array}$ & $\begin{array}{c}\text { Total } \\
\text { Coverage } \\
\text { Duration } \\
\text { L.B. [s] } \\
\text { (Estimated) }\end{array}$ & $\begin{array}{c}\text { Total } \\
\text { Coverage } \\
\text { Duration } \\
\text { L.B. [s] } \\
\text { (Explicit) }\end{array}$ & $\begin{array}{c}\text { Total } \\
\text { Coverage } \\
\text { Duration } \\
\text { U.B. [s] } \\
\text { (Explicit) }\end{array}$ & $\begin{array}{c}\text { Total } \\
\text { Coverage } \\
\text { Duration } \\
\text { U.B. [s] } \\
\text { (Estimated) }\end{array}$ \\
\hline $90.0 \mathrm{~N} ; 77.0 \mathrm{~W}$ & 0 & 0 & 0 & 0 & 0 & 0 & 0 & 0 & 0 \\
\hline $85.0 \mathrm{~N} ; 77.0 \mathrm{~W}$ & 0 & 0 & 0 & 0 & 0 & 0 & 0 & 0 & 0 \\
\hline $80.0 \mathrm{~N} ; 77.0 \mathrm{~W}$ & 0 & 0 & 0 & 0 & 0 & 0 & 0 & 0 & 0 \\
\hline $75.0 \mathrm{~N} ; 77.0 \mathrm{~W}$ & 3184 & 4200 & 1 & 2 & 3 & 2400 & 5254 & 5374 & 9554 \\
\hline $70.0 \mathrm{~N} ; 77.0 \mathrm{~W}$ & 3840 & 5400 & 1 & 3 & 3 & 3900 & 10144 & 10324 & 14043 \\
\hline $65.0 \mathrm{~N} ; 77.0 \mathrm{~W}$ & 4770 & 6237 & 2 & 3 & 4 & 5637 & 12574 & 12754 & 16737 \\
\hline $60.0 \mathrm{~N} ; 77.0 \mathrm{~W}$ & 5370 & 6834 & 2 & 3 & 4 & 7463 & 14104 & 14284 & 18834 \\
\hline $55.0 \mathrm{~N} ; 77.0 \mathrm{~W}$ & 5790 & 7429 & 2 & 3 & 4 & 9300 & 15154 & 15334 & 20029 \\
\hline $50.0 \mathrm{~N} ; 77.0 \mathrm{~W}$ & 6090 & 7500 & 3 & 3 & 5 & 11026 & 15874 & 16054 & 21226 \\
\hline $45.0 \mathrm{~N} ; 77.0 \mathrm{~W}$ & 6300 & 8024 & 3 & 3 & 5 & 11924 & 16384 & 16564 & 21824 \\
\hline $40.0 \mathrm{~N} ; 77.0 \mathrm{~W}$ & 6450 & 8021 & 3 & 3 & 5 & 12821 & 16714 & 16894 & 22972 \\
\hline $35.0 \mathrm{~N} ; 77.0 \mathrm{~W}$ & 6510 & 8100 & 3 & 3 & 5 & 13718 & 16864 & 17044 & 22966 \\
\hline $30.0 \mathrm{~N} ; 77.0 \mathrm{~W}$ & 6540 & 8100 & 3 & 3 & 5 & 13800 & 16894 & 17074 & 23862 \\
\hline $25.0 \mathrm{~N} ; 77.0 \mathrm{~W}$ & 6510 & 8100 & 3 & 3 & 5 & 14014 & 16774 & 16954 & 24158 \\
\hline $20.0 \mathrm{~N} ; 77.0 \mathrm{~W}$ & 6420 & 8100 & 3 & 4 & 5 & 13500 & 17284 & 17524 & 23611 \\
\hline $15.0 \mathrm{~N} ; 77.0 \mathrm{~W}$ & 6300 & 8100 & 3 & 4 & 5 & 12900 & 17194 & 17434 & 23908 \\
\hline $10.0 \mathrm{~N} ; 77.0 \mathrm{~W}$ & 6060 & 8100 & 3 & 4 & 5 & 12507 & 16714 & 16954 & 23307 \\
\hline $5.0 \mathrm{~N} ; 77.0 \mathrm{~W}$ & 5760 & 7800 & 3 & 4 & 5 & 11902 & 15934 & 16174 & 22702 \\
\hline $0.0 \mathrm{~N} ; 77.0 \mathrm{~W}$ & 5370 & 7700 & 3 & 5 & 5 & 10800 & 15394 & 15694 & 22100 \\
\hline $5.0 \mathrm{~S} ; 77.0 \mathrm{~W}$ & 4830 & 7200 & 3 & 5 & 5 & 9600 & 14194 & 14494 & 21196 \\
\hline
\end{tabular}




\begin{tabular}{|c|c|c|c|c|c|c|c|c|c|}
\hline Target & $\begin{array}{l}\text { Maximum } \\
\text { Coverage } \\
\text { Window } \\
\text { Duration [s] } \\
\text { (Explicit) }\end{array}$ & $\begin{array}{c}\text { Maximum } \\
\text { Coverage } \\
\text { Window } \\
\text { Duration [s] } \\
\text { (Estimated) }\end{array}$ & $\begin{array}{c}\text { Number of } \\
\text { Windows } \\
\text { L.B. } \\
\text { (Estimated) }\end{array}$ & $\begin{array}{l}\text { Number of } \\
\text { Windows } \\
\text { (Explicit) }\end{array}$ & $\begin{array}{c}\text { Number of } \\
\text { Windows } \\
\text { L.B. } \\
\text { (Estimated) }\end{array}$ & $\begin{array}{c}\text { Total } \\
\text { Coverage } \\
\text { Duration } \\
\text { L.B. [s] } \\
\text { (Estimated) }\end{array}$ & $\begin{array}{c}\text { Total } \\
\text { Coverage } \\
\text { Duration } \\
\text { L.B. [s] } \\
\text { (Explicit) }\end{array}$ & $\begin{array}{c}\text { Total } \\
\text { Coverage } \\
\text { Duration } \\
\text { U.B. [s] } \\
\text { (Explicit) }\end{array}$ & $\begin{array}{c}\text { Total } \\
\text { Coverage } \\
\text { Duration } \\
\text { U.B. [s] } \\
\text { (Estimated) }\end{array}$ \\
\hline $10.0 \mathrm{~S} ; 77.0 \mathrm{~W}$ & 4170 & 6600 & 4 & 5 & 5 & 8293 & 12484 & 12784 & 19093 \\
\hline $15.0 \mathrm{~S} ; 77.0 \mathrm{~W}$ & 3300 & 5400 & 4 & 5 & 6 & 6187 & 9994 & 10294 & 17100 \\
\hline $20.0 \mathrm{~S} ; 77.0 \mathrm{~W}$ & 2220 & 3000 & 4 & 5 & 12 & 2700 & 6424 & 6724 & 17900 \\
\hline $25.0 \mathrm{~S} ; 77.0 \mathrm{~W}$ & 1320 & 1800 & 3 & 4 & 6 & 1500 & 2670 & 2910 & 6000 \\
\hline $30.0 \mathrm{~S} ; 77.0 \mathrm{~W}$ & 570 & 1384 & 2 & 3 & 6 & 0 & 1110 & 1290 & 5283 \\
\hline $35.0 \mathrm{~S} ; 77.0 \mathrm{~W}$ & 240 & 898 & 0 & 2 & 4 & 0 & 240 & 360 & 2931 \\
\hline $40.0 \mathrm{~S} ; 77.0 \mathrm{~W}$ & 0 & 0 & 0 & 0 & 0 & 0 & 0 & 0 & 0 \\
\hline $45.0 \mathrm{~S} ; 77.0 \mathrm{~W}$ & 0 & 0 & 0 & 0 & 0 & 0 & 0 & 0 & 0 \\
\hline $50.0 \mathrm{~S} ; 77.0 \mathrm{~W}$ & 0 & 0 & 0 & 0 & 0 & 0 & 0 & 0 & 0 \\
\hline $55.0 \mathrm{~S} ; 77.0 \mathrm{~W}$ & 0 & 0 & 0 & 0 & 0 & 0 & 0 & 0 & 0 \\
\hline $60.0 \mathrm{~S} ; 77.0 \mathrm{~W}$ & 0 & 0 & 0 & 0 & 0 & 0 & 0 & 0 & 0 \\
\hline $65.0 \mathrm{~S} ; 77.0 \mathrm{~W}$ & 0 & 0 & 0 & 0 & 0 & 0 & 0 & 0 & 0 \\
\hline $70.0 \mathrm{~S} ; 77.0 \mathrm{~W}$ & 0 & 0 & 0 & 0 & 0 & 0 & 0 & 0 & 0 \\
\hline $75.0 \mathrm{~S} ; 77.0 \mathrm{~W}$ & 0 & 0 & 0 & 0 & 0 & 0 & 0 & 0 & 0 \\
\hline $80.0 \mathrm{~S} ; 77.0 \mathrm{~W}$ & 0 & 0 & 0 & 0 & 0 & 0 & 0 & 0 & 0 \\
\hline $85.0 \mathrm{~S} ; 77.0 \mathrm{~W}$ & 0 & 0 & 0 & 0 & 0 & 0 & 0 & 0 & 0 \\
\hline $90.0 \mathrm{~S} ; 77.0 \mathrm{~W}$ & 0 & 0 & 0 & 0 & 0 & 0 & 0 & 0 & 0 \\
\hline
\end{tabular}


Table 24: Comparison of explicit coverage versus estimated coverage across Washington, D.C.'s latitude over the first sidereal day

\begin{tabular}{|c|c|c|c|c|c|c|c|c|c|}
\hline Target & $\begin{array}{l}\text { Maximum } \\
\text { Coverage } \\
\text { Window } \\
\text { Duration [s] } \\
\text { (Explicit) }\end{array}$ & $\begin{array}{l}\text { Maximum } \\
\text { Coverage } \\
\text { Window } \\
\text { Duration [s] } \\
\text { (Estimated) }\end{array}$ & $\begin{array}{c}\text { Number of } \\
\text { Windows } \\
\text { L.B. } \\
\text { (Estimated) }\end{array}$ & $\begin{array}{l}\text { Number of } \\
\text { Windows } \\
\text { (Explicit) }\end{array}$ & $\begin{array}{l}\text { Number of } \\
\text { Windows } \\
\text { L.B. } \\
\text { (Estimated) }\end{array}$ & $\begin{array}{c}\text { Total } \\
\text { Coverage } \\
\text { Duration } \\
\text { L.B. [s] } \\
\text { (Estimated) }\end{array}$ & $\begin{array}{c}\text { Total } \\
\text { Coverage } \\
\text { Duration } \\
\text { L.B. [s] } \\
\text { (Explicit) }\end{array}$ & $\begin{array}{c}\text { Total } \\
\text { Coverage } \\
\text { Duration } \\
\text { U.B. [s] } \\
\text { (Explicit) }\end{array}$ & $\begin{array}{c}\text { Total } \\
\text { Coverage } \\
\text { Duration } \\
\text { U.B. [s] } \\
\text { (Estimated) }\end{array}$ \\
\hline $38.9 \mathrm{~N} ; 180.0 \mathrm{E}$ & 7470 & 7800 & 2 & 3 & 4 & 13200 & 17970 & 18150 & 23088 \\
\hline $38.9 \mathrm{~N} ; 175.0 \mathrm{~W}$ & 7440 & 7800 & 2 & 4 & 4 & 12900 & 19470 & 19710 & 23685 \\
\hline $38.9 \mathrm{~N} ; 170.0 \mathrm{~W}$ & 7380 & 7800 & 2 & 4 & 4 & 12900 & 20130 & 20370 & 23382 \\
\hline $38.9 \mathrm{~N} ; 165.0 \mathrm{~W}$ & 7290 & 7800 & 2 & 4 & 4 & 12900 & 20430 & 20670 & 23679 \\
\hline $38.9 \mathrm{~N} ; 160.0 \mathrm{~W}$ & 7170 & 7800 & 2 & 4 & 4 & 12300 & 20460 & 20700 & 23675 \\
\hline $38.9 \mathrm{~N} ; 155.0 \mathrm{~W}$ & 6990 & 7800 & 2 & 4 & 4 & 12300 & 19980 & 20220 & 23972 \\
\hline $38.9 \mathrm{~N} ; 150.0 \mathrm{~W}$ & 7140 & 7800 & 2 & 3 & 4 & 12300 & 18840 & 19020 & 23369 \\
\hline $38.9 \mathrm{~N} ; 145.0 \mathrm{~W}$ & 7290 & 7800 & 3 & 3 & 5 & 11966 & 19200 & 19380 & 23271 \\
\hline $38.9 \mathrm{~N} ; 140.0 \mathrm{~W}$ & 7380 & 7800 & 3 & 3 & 5 & 12862 & 19380 & 19560 & 22462 \\
\hline $38.9 \mathrm{~N} ; 135.0 \mathrm{~W}$ & 7440 & 7800 & 3 & 3 & 5 & 13159 & 19440 & 19620 & 22759 \\
\hline $38.9 \mathrm{~N} ; 130.0 \mathrm{~W}$ & 7470 & 7800 & 3 & 3 & 5 & 13756 & 19114 & 19294 & 22456 \\
\hline $38.9 \mathrm{~N} ; 125.0 \mathrm{~W}$ & 7470 & 7800 & 3 & 3 & 5 & 12900 & 18604 & 18784 & 22752 \\
\hline $38.9 \mathrm{~N} ; 120.0 \mathrm{~W}$ & 7440 & 7800 & 3 & 4 & 5 & 12300 & 19714 & 19954 & 22749 \\
\hline $38.9 \mathrm{~N} ; 115.0 \mathrm{~W}$ & 7350 & 7800 & 3 & 4 & 5 & 11646 & 19954 & 20194 & 23346 \\
\hline $38.9 \mathrm{~N} ; 110.0 \mathrm{~W}$ & 7260 & 7800 & 2 & 4 & 4 & 11643 & 19804 & 20044 & 23343 \\
\hline $38.9 \mathrm{~N} ; 105.0 \mathrm{~W}$ & 7140 & 7800 & 2 & 4 & 4 & 12000 & 19384 & 19624 & 23639 \\
\hline $38.9 \mathrm{~N} ; 100.0 \mathrm{~W}$ & 6960 & 8036 & 2 & 4 & 4 & 12000 & 18484 & 18724 & 23636 \\
\hline $38.9 \mathrm{~N} ; 95.0 \mathrm{~W}$ & 6750 & 7800 & 2 & 3 & 4 & 12000 & 17224 & 17404 & 23400 \\
\hline $38.9 \mathrm{~N} ; 90.0 \mathrm{~W}$ & 6510 & 8030 & 2 & 3 & 4 & 11700 & 17224 & 17404 & 23630 \\
\hline $38.9 \mathrm{~N} ; 85.0 \mathrm{~W}$ & 6240 & 8026 & 3 & 3 & 4 & 11400 & 17164 & 17344 & 23271 \\
\hline
\end{tabular}




\begin{tabular}{|c|c|c|c|c|c|c|c|c|c|}
\hline Target & $\begin{array}{c}\text { Maximum } \\
\text { Coverage } \\
\text { Window } \\
\text { Duration [s] } \\
\text { (Explicit) }\end{array}$ & $\begin{array}{c}\text { Maximum } \\
\text { Coverage } \\
\text { Window } \\
\text { Duration [s] } \\
\text { (Estimated) }\end{array}$ & $\begin{array}{c}\text { Number of } \\
\text { Windows } \\
\text { L.B. } \\
\text { (Estimated) }\end{array}$ & $\begin{array}{l}\text { Number of } \\
\text { Windows } \\
\text { (Explicit) }\end{array}$ & $\begin{array}{c}\text { Number of } \\
\text { Windows } \\
\text { L.B. } \\
\text { (Estimated) }\end{array}$ & $\begin{array}{c}\text { Total } \\
\text { Coverage } \\
\text { Duration } \\
\text { L.B. [s] } \\
\text { (Estimated) }\end{array}$ & $\begin{array}{c}\text { Total } \\
\text { Coverage } \\
\text { Duration } \\
\text { L.B. [s] } \\
\text { (Explicit) }\end{array}$ & $\begin{array}{c}\text { Total } \\
\text { Coverage } \\
\text { Duration } \\
\text { U.B. [s] } \\
\text { (Explicit) }\end{array}$ & $\begin{array}{c}\text { Total } \\
\text { Coverage } \\
\text { Duration } \\
\text { U.B. [s] } \\
\text { (Estimated) }\end{array}$ \\
\hline $38.9 \mathrm{~N} ; 80.0 \mathrm{~W}$ & 6300 & 8023 & 3 & 3 & 5 & 12223 & 16954 & 17134 & 22971 \\
\hline $38.9 \mathrm{~N} ; 75.0 \mathrm{~W}$ & 6570 & 8020 & 3 & 3 & 5 & 12820 & 16594 & 16774 & 22420 \\
\hline $38.9 \mathrm{~N} ; 70.0 \mathrm{~W}$ & 6810 & 8016 & 3 & 3 & 5 & 13716 & 16174 & 16354 & 22716 \\
\hline $38.9 \mathrm{~N} ; 65.0 \mathrm{~W}$ & 6990 & 7800 & 3 & 4 & 5 & 13713 & 17464 & 17704 & 22413 \\
\hline $38.9 \mathrm{~N} ; 60.0 \mathrm{~W}$ & 7170 & 7800 & 3 & 4 & 5 & 12600 & 17704 & 17944 & 22200 \\
\hline $38.9 \mathrm{~N} ; 55.0 \mathrm{~W}$ & 7290 & 7800 & 3 & 4 & 5 & 12300 & 17524 & 17764 & 23007 \\
\hline $38.9 \mathrm{~N} ; 50.0 \mathrm{~W}$ & 7380 & 7800 & 2 & 4 & 4 & 12000 & 17044 & 17284 & 23303 \\
\hline $38.9 \mathrm{~N} ; 45.0 \mathrm{~W}$ & 7440 & 7800 & 2 & 4 & 4 & 12200 & 16054 & 16294 & 23600 \\
\hline $38.9 \mathrm{~N} ; 40.0 \mathrm{~W}$ & 7470 & 7800 & 2 & 3 & 4 & 12600 & 14974 & 15154 & 23597 \\
\hline $38.9 \mathrm{~N} ; 35.0 \mathrm{~W}$ & 7470 & 7800 & 2 & 3 & 4 & 12600 & 14884 & 15064 & 23700 \\
\hline $38.9 \mathrm{~N} ; 30.0 \mathrm{~W}$ & 7410 & 7800 & 2 & 3 & 4 & 12900 & 14674 & 14854 & 23290 \\
\hline $38.9 \mathrm{~N} ; 25.0 \mathrm{~W}$ & 7350 & 7800 & 2 & 3 & 4 & 12900 & 14344 & 14524 & 22800 \\
\hline $38.9 \mathrm{~N} ; 20.0 \mathrm{~W}$ & 7230 & 7800 & 2 & 3 & 4 & 12900 & 13864 & 14044 & 22384 \\
\hline $38.9 \mathrm{~N} ; 15.0 \mathrm{~W}$ & 7110 & 7800 & 2 & 3 & 4 & 12900 & 14190 & 14370 & 21900 \\
\hline $38.9 \mathrm{~N} ; 10.0 \mathrm{~W}$ & 7050 & 7800 & 2 & 3 & 4 & 13200 & 15870 & 16050 & 21294 \\
\hline $38.9 \mathrm{~N} ; 5.0 \mathrm{~W}$ & 7200 & 7800 & 3 & 3 & 5 & 14274 & 16740 & 16920 & 20997 \\
\hline $38.9 \mathrm{~N} ; 0.0 \mathrm{E}$ & 7320 & 7800 & 3 & 3 & 4 & 14700 & 17310 & 17490 & 19500 \\
\hline $38.9 \mathrm{~N} ; 5.0 \mathrm{E}$ & 7410 & 7800 & 3 & 3 & 4 & 14700 & 17700 & 17880 & 19500 \\
\hline $38.9 \mathrm{~N} ; 10.0 \mathrm{E}$ & 7440 & 7800 & 3 & 3 & 4 & 14700 & 17940 & 18120 & 19500 \\
\hline $38.9 \mathrm{~N} ; 15.0 \mathrm{E}$ & 7470 & 7800 & 3 & 3 & 4 & 14700 & 18030 & 18210 & 19500 \\
\hline $38.9 \mathrm{~N} ; 20.0 \mathrm{E}$ & 7470 & 7800 & 3 & 3 & 4 & 14700 & 18000 & 18180 & 19500 \\
\hline
\end{tabular}




\begin{tabular}{|c|c|c|c|c|c|c|c|c|c|}
\hline Target & $\begin{array}{c}\text { Maximum } \\
\text { Coverage } \\
\text { Window } \\
\text { Duration [s] } \\
\text { (Explicit) }\end{array}$ & $\begin{array}{c}\text { Maximum } \\
\text { Coverage } \\
\text { Window } \\
\text { Duration [s] } \\
\text { (Estimated) }\end{array}$ & $\begin{array}{c}\text { Number of } \\
\text { Windows } \\
\text { L.B. } \\
\text { (Estimated) }\end{array}$ & $\begin{array}{l}\text { Number of } \\
\text { Windows } \\
\text { (Explicit) }\end{array}$ & $\begin{array}{c}\text { Number of } \\
\text { Windows } \\
\text { L.B. } \\
\text { (Estimated) }\end{array}$ & $\begin{array}{c}\text { Total } \\
\text { Coverage } \\
\text { Duration } \\
\text { L.B. [s] } \\
\text { (Estimated) }\end{array}$ & $\begin{array}{c}\text { Total } \\
\text { Coverage } \\
\text { Duration } \\
\text { L.B. [s] } \\
\text { (Explicit) }\end{array}$ & $\begin{array}{c}\text { Total } \\
\text { Coverage } \\
\text { Duration } \\
\text { U.B. [s] } \\
\text { (Explicit) }\end{array}$ & $\begin{array}{c}\text { Total } \\
\text { Coverage } \\
\text { Duration } \\
\text { U.B. [s] } \\
\text { (Estimated) }\end{array}$ \\
\hline $38.9 \mathrm{~N} ; 25.0 \mathrm{E}$ & 7410 & 7800 & 3 & 3 & 4 & 14700 & 17760 & 17940 & 19500 \\
\hline $38.9 \mathrm{~N} ; 30.0 \mathrm{E}$ & 7350 & 7800 & 3 & 3 & 4 & 14700 & 17370 & 17550 & 19500 \\
\hline $38.9 \mathrm{~N} ; 35.0 \mathrm{E}$ & 7230 & 7800 & 3 & 3 & 4 & 14700 & 16770 & 16950 & 19500 \\
\hline $38.9 \mathrm{~N} ; 40.0 \mathrm{E}$ & 7080 & 7800 & 3 & 4 & 4 & 14700 & 16650 & 16890 & 19500 \\
\hline $38.9 \mathrm{~N} ; 45.0 \mathrm{E}$ & 7080 & 7800 & 3 & 3 & 4 & 14700 & 16050 & 16230 & 19500 \\
\hline $38.9 \mathrm{~N} ; 50.0 \mathrm{E}$ & 7200 & 7800 & 3 & 3 & 4 & 14700 & 16830 & 17010 & 19500 \\
\hline $38.9 \mathrm{~N} ; 55.0 \mathrm{E}$ & 7350 & 7800 & 3 & 3 & 4 & 14700 & 17400 & 17580 & 19500 \\
\hline $38.9 \mathrm{~N} ; 60.0 \mathrm{E}$ & 7410 & 7800 & 3 & 3 & 4 & 14700 & 17760 & 17940 & 19500 \\
\hline $38.9 \mathrm{~N} ; 65.0 \mathrm{E}$ & 7470 & 7800 & 3 & 3 & 4 & 14700 & 17970 & 18150 & 19500 \\
\hline $38.9 \mathrm{~N} ; 70.0 \mathrm{E}$ & 7470 & 7800 & 3 & 3 & 4 & 14700 & 18030 & 18210 & 19500 \\
\hline $38.9 \mathrm{~N} ; 75.0 \mathrm{E}$ & 7440 & 7800 & 3 & 3 & 4 & 14700 & 17910 & 18090 & 19500 \\
\hline $38.9 \mathrm{~N} ; 80.0 \mathrm{E}$ & 7410 & 7800 & 3 & 3 & 4 & 14700 & 17670 & 17850 & 19500 \\
\hline $38.9 \mathrm{~N} ; 85.0 \mathrm{E}$ & 7320 & 7800 & 3 & 3 & 4 & 14700 & 17250 & 17430 & 19500 \\
\hline $38.9 \mathrm{~N} ; 90.0 \mathrm{E}$ & 7200 & 7800 & 3 & 3 & 4 & 14700 & 16680 & 16860 & 19500 \\
\hline $38.9 \mathrm{~N} ; 95.0 \mathrm{E}$ & 7050 & 7800 & 3 & 4 & 4 & 14700 & 16770 & 17010 & 19500 \\
\hline $38.9 \mathrm{~N} ; 100.0 \mathrm{E}$ & 7110 & 7800 & 3 & 3 & 4 & 14700 & 16200 & 16380 & 19500 \\
\hline $38.9 \mathrm{~N} ; 105.0 \mathrm{E}$ & 7230 & 7800 & 3 & 3 & 4 & 14700 & 16890 & 17070 & 19500 \\
\hline $38.9 \mathrm{~N} ; 110.0 \mathrm{E}$ & 7320 & 7800 & 3 & 3 & 4 & 14700 & 17400 & 17580 & 19500 \\
\hline $38.9 \mathrm{~N} ; 115.0 \mathrm{E}$ & 7410 & 7800 & 3 & 3 & 4 & 14700 & 17760 & 17940 & 19500 \\
\hline $38.9 \mathrm{~N} ; 120.0 \mathrm{E}$ & 7470 & 7800 & 3 & 3 & 4 & 14700 & 17970 & 18150 & 19500 \\
\hline $38.9 \mathrm{~N} ; 125.0 \mathrm{E}$ & 7470 & 7800 & 3 & 3 & 4 & 14700 & 17970 & 18150 & 19500 \\
\hline
\end{tabular}




\begin{tabular}{|c|c|c|c|c|c|c|c|c|c|}
\hline Target & $\begin{array}{c}\text { Maximum } \\
\text { Coverage } \\
\text { Window } \\
\text { Duration [s] } \\
\text { (Explicit) }\end{array}$ & $\begin{array}{l}\text { Maximum } \\
\text { Coverage } \\
\text { Window } \\
\text { Duration [s] } \\
\text { (Estimated) }\end{array}$ & $\begin{array}{c}\text { Number of } \\
\text { Windows } \\
\text { L.B. } \\
\text { (Estimated) }\end{array}$ & $\begin{array}{l}\text { Number of } \\
\text { Windows } \\
\text { (Explicit) }\end{array}$ & $\begin{array}{c}\text { Number of } \\
\text { Windows } \\
\text { L.B. } \\
\text { (Estimated) }\end{array}$ & $\begin{array}{c}\text { Total } \\
\text { Coverage } \\
\text { Duration } \\
\text { L.B. [s] } \\
\text { (Estimated) }\end{array}$ & $\begin{array}{c}\text { Total } \\
\text { Coverage } \\
\text { Duration } \\
\text { L.B. [s] } \\
\text { (Explicit) }\end{array}$ & $\begin{array}{c}\text { Total } \\
\text { Coverage } \\
\text { Duration } \\
\text { U.B. [s] } \\
\text { (Explicit) }\end{array}$ & $\begin{array}{c}\text { Total } \\
\text { Coverage } \\
\text { Duration } \\
\text { U.B. [s] } \\
\text { (Estimated) }\end{array}$ \\
\hline $38.9 \mathrm{~N} ; 130.0 \mathrm{E}$ & 7440 & 7800 & 3 & 3 & 4 & 14700 & 17910 & 18090 & 19500 \\
\hline $38.9 \mathrm{~N} ; 135.0 \mathrm{E}$ & 7380 & 7800 & 3 & 3 & 4 & 14700 & 17640 & 17820 & 19500 \\
\hline $38.9 \mathrm{~N} ; 140.0 \mathrm{E}$ & 7290 & 7800 & 3 & 3 & 4 & 14700 & 17190 & 17370 & 19500 \\
\hline $38.9 \mathrm{~N} ; 145.0 \mathrm{E}$ & 7200 & 7800 & 3 & 3 & 4 & 14700 & 16590 & 16770 & 19500 \\
\hline $38.9 \mathrm{~N} ; 150.0 \mathrm{E}$ & 7020 & 7800 & 3 & 4 & 4 & 14700 & 16890 & 17130 & 19500 \\
\hline $38.9 \mathrm{~N} ; 155.0 \mathrm{E}$ & 7140 & 7800 & 3 & 3 & 4 & 14700 & 16320 & 16500 & 19500 \\
\hline $38.9 \mathrm{~N} ; 160.0 \mathrm{E}$ & 7260 & 7800 & 3 & 3 & 4 & 14700 & 17010 & 17190 & 19500 \\
\hline $38.9 \mathrm{~N} ; 165.0 \mathrm{E}$ & 7350 & 7800 & 3 & 3 & 5 & 14273 & 17490 & 17670 & 20698 \\
\hline $38.9 \mathrm{~N} ; 170.0 \mathrm{E}$ & 7440 & 7800 & 3 & 3 & 4 & 13200 & 17850 & 18030 & 21595 \\
\hline $38.9 \mathrm{~N} ; 175.0 \mathrm{E}$ & 7470 & 7800 & 2 & 3 & 4 & 13200 & 17970 & 18150 & 22192 \\
\hline
\end{tabular}





\section{References}

${ }^{1}$ Vallado, D.A., and McClain, W.D., Fundamentals of Astrodynamics and Applications, McGraw-Hill Companies, Inc., New York, 1997, pp.21, 209-215, 384.

2Jet Propulsion Laboratory, "Astrodynamical Constants," 2007, URL:http://ssd.jpl.nasa.gov/astro_constants.html [cited 25 Jun.2007].

${ }^{3}$ Luders, R.D., "Satellite Networks for Continuous Zonal Coverage," ARS Journal, Vol.31, Feb.1961, pp.179-184.

${ }^{4}$ Rider, L., "Analytic Design of Satellite Constellations for Zonal Earth Coverage Using Circular Orbits," Journal of Astronautical Sciences, Vol.34, No.1, Jan.-Mar.1986, pp.31-64.

${ }^{5}$ Adams, W.S., and Rider, L., "Circular Polar Constellations Providing Continuous Single or Multiple Coverage Above a Specified Latitude," Journal of Astronautical Sciences, Vol.35, Apr.-Jun.1987, pp.155-192.

${ }^{6}$ Walker, J., "Circular Orbit Patterns Providing Whole Earth Coverage," Royal Aircraft Establishment, Technical Report 70211, Nov.1970.

${ }^{7}$ Mozhaev, G.V., "The Problem of Continuous Earth Coverage and the Kinematically Regular Satellite Networks," Cosmic Research, Vol.1, Nov.Dec.1972.

${ }^{8}$ Mozhaev, G.V., "The Problem of Continuous Earth Coverage and the Kinematically Regular Satellite Networks," Cosmic Research, Vol.2, Jan.Feb.1973.

${ }^{9}$ Beste, D.C., "Design of Satellite Constellations for Optimal Continuous Coverage," IEEE Transactions on Aerospace and Electronic Systems, Vol.AES14, May 1978, pp.466-473.

${ }^{10}$ Rider, L., "Optimized Polar Constellations for Redundant Earth Coverage," Journal of Astronautical Sciences, Vol.34, Jan.-Mar.1986, pp.31-64.

${ }^{11}$ Rowell, L.N., "Satellite Constellations for Worldwide and Hemisphere Coverage," RAND Corporation, N-1432-DCA, Dec.1979.

${ }^{12}$ Draim, J.E., "Three- and Four-Satellite Continuous Coverage Constellations," Journal of Guidance, Control and Dynamics, Vol.8, No.6, Nov.Dec.1985.

${ }^{13}$ Draim, J.E., "A Common-Period Four-Satellite Continuous Global Coverage Constellation," Journal of Guidance, Control and Dynamics, Vol.10, No.5, Sep.Oct.1987, pp.492-499. 
${ }^{14}$ Palmerini, G.B., and Graziani, F., "Polar Elliptic Orbits for Global Coverage Constellations," Astrodynamics Conference, AIAA-94-3720-CP, AIAA, Scottsdale, AZ, 1-3 Aug.1994, pp.120-129.

${ }^{15} \mathrm{Ma}, \mathrm{D} .$, and Hsu, W., "Exact Design of Partial Coverage Satellite Constellations Over Oblate Earth," AIAA/AAS Astrodynamics Conference, Paper No.A94-30483, Scottsdale, AZ, 1-3 Aug.1994.

${ }^{16}$ Castiel, D., Brosius, J.W., and Draim, J.E., "Ellipso: Coverage Optimization Using Elliptic Orbits," Fifteenth International Communications Satellite Systems Conference, AIAA-94-1098-CP, AIAA, San Diego, CA, 28 Feb.-3 Mar.1994, pp.1235-1241.

${ }^{17}$ Hanson, J.M., Evans, M.J., and Turner, R.E., "Designing Good Partial Coverage Satellite Constellations," Journal of the Astronautical Sciences, Vol.40, No.2, Apr.-Jun.1992, pp.215-239.

${ }^{18}$ Mora, M.B., Munoz, J.P., and Dutruel-Lecohier, G., "Orion - A Constellation Mission Analysis Tool," Mission Design and Implementation of Satellite Constellations: Proceedings of an International Workshop, held in Toulouse, France, 17-19 Nov.1997, edited by J.C. van der Halnternational Astronautical Federation, Kluwer Academic Publishers, Dordrecht, The Netherlands, 1998, p.373.

${ }^{19}$ George, E., "Optimization of Satellite Constellations for Discontinuous Global Coverage Using Genetic Algorithms," AAS/AIAA Astrodynamics Specialist Conference, Paper No.AAS 97-621, AAS/AIAA, Sun Valley, ID, Aug.1997.

${ }^{20}$ Williams, E.A., Crossley, W.A., and Lang, T.J., "Average and Maximum Revisit Time Trade Studies for Satellite Constellations Using a Multiobjective Genetic Algorithm," Journal of the Astronautical Sciences, Vol.49, No.3, Jul.Sep. 2001, pp.385-400.

${ }^{21}$ Lang, T.J., "A Parametric Examination of Satellite Constellations to Minimize Revisit Time for Low Earth Orbits Using a Genetic Algorithm," Advances in the Astronautical Sciences, edited by D.B. Spencer, C.C. Seybold, A.K. Misra, and R.J. Lisowski, AAS/AIAA Astrodynamics 2001, Univelt, Inc., San Diego, CA, 2001, pp.625-640.

${ }^{22}$ Ferringer, M.P, Clifton, R.S., and Thompson, T.G., "Constellation Design with Parallel Multi-Objective Evolutionary Computation," AIAA/AAS Astrodynamics Specialist Conference and Exhibit, 21-24 Aug. 2006, Keystone, Colorado, AIAA-2006-6015, AIAA, 2006.

${ }^{23}$ Shen $\mathrm{H}$. and Tsiotras, $\mathrm{P}$, "Peer-to-Peer Refueling for Circular Satellite Constellations," Journal of Guidance, Control, and Dynamics, Vol. 28, No. 6, 2005, pp.1220-1230.

${ }^{24}$ de Weck, O., de Neufville, R., Chaize, M., "Staged Deployment of Communications Satellite Constellations in Low Earth Orbit," Journal of 
Aerospace Computing, Information, and Communications, Vol. 1, , March 2004, pp.119-136.

${ }^{25}$ Siddiqi, A., Mellein, J., de Weck, O.L., "Optimal Reconfigurations for Increasing Capacity of Communication Satellite Constellations," 46th AIAA/ASME/ASCE/AHS/ASC Structures, Structural Dynamics and Materials Conference, Austin, Texas, AIAA, Apr. 18-21, 2005.

${ }^{26}$ Janson, S.W., "Electric Propulsion for Low Earth Orbiting Constellation Morphing," 38th AIAA/ASME/SAE/ASEE Joint Propulsion Conference \& Exhibit, 7-10 July 2002, Indianapolis, Indiana, AIAA-2002-3669, AIAA, 2002.

${ }^{27}$ de Weck, O.L., Scialom, U., and Siddiqi, A., "Optimal Reconfiguration of Satellite Constellations with the Auction Algorithm," 22nd AIAA International Communications Satellite Systems Conference \& Exhibit, AIAA 2004-3162, AIAA, Monterey, CA, 9-12 May 2004.

${ }^{28}$ Montenbruck, O., and Gill, E., Satellite Orbits: Models, Methods, Applications, Springer-Verlag, Berlin, 2000, pp.56-68.

${ }^{29}$ National Aeronautics and Space Administration Goddard Space Flight Center, and National Imagery and Mapping Agency, Ohio State University, "EGM96: The NASA GSFC and NIMA Joint Geopotential Model," Jul.1998, URL:http://cddis.gsfc.nasa.gov/926/egm96/egm96.html [cited 8 Feb.2007].

${ }^{30}$ U.S. Naval Observatory, Rutherford Appleton Laboratory, Astronomical Almanac, U.S. Government Printing Office, Washington, DC, 1984, p.S15.

${ }^{31}$ Sutton, G.P., and Biblarz, O., Rocket Propulsion Elements, Seventh Edition, John Wiley \& Sons, New York, 2001, pp.27-44, 102-106.

${ }^{32}$ Epperson, J.F., An Introduction to Numerical Methods and Analysis, John Wiley \& Sons, New York, 2002, pp.370-382.

${ }^{33}$ U.S. Naval Observatory, Rutherford Appleton Laboratory, The Astronomical Almanac for the Year 2001, U.S. Government Printing Office, Washington, DC, 2000, p.E88.

${ }^{34}$ Tang, C.H., "An Exact Solution for Orbit View-Periods from a Station on a Tri-Axial Ellipsoidal Planet," Astrodynamics Conference, AIAA-86-2068, AIAA and AAS, Williamsburg, VA, 18-20 Aug.1986.

${ }^{35}$ Wertz, J.R., Meissinger, H.F., Newman, L.K., and Smit, G.N., Mission Geometry; Orbit and Constellation Design and Management, Microcosm Press, El Segundo, CA, 2001, pp.468, 472, 483.

${ }^{36}$ Hayes, E., "An Algorithm for the Computation of Coverage Area by Earth Observing Satellites," Astrodynamics Conference, AIAA-1986-2067, AIAA, New York, 18-20 Aug. 1986, pp.121-126. 
${ }^{37}$ Middour, J.W., "An efficient technique for computation of satellite earth coverage," Aerospace Sciences Meeting, 27th, AIAA-1989-0452, AIAA, Reno, NV, 9-12 Jan.1989, pp.1-7.

${ }^{38}$ Sauter, L.M., "Satellite Constellation Design for Mid-Course Ballistic Missile Interception," MSc Thesis, Massachusetts Institute of Technology, Cambridge, MA, 2004.

${ }^{39}$ Bate, R.R., Mueller, D.D., and White, J.E., Fundamentals of Astrodynamics, Dover Publications, Inc., New York, 1971, pp. 11-14.

${ }^{40}$ Prussing, J.E., and Conway, B.A., Orbital Mechanics, Oxford University Press, New York, 1993, pp.165-169.

${ }^{41}$ Schaub, H., and Alfriend, K.T., "Impulsive Feedback Control to Establish Mean Orbit Elements of Spacecraft Formations," Journal of Guidance, Navigation and Control, Vol.24, No.4, Aug. 2001, pp.739-745.

${ }^{42}$ Battin, R.H., An Introduction to the Mathematics and Methods of Astrodynamics, AIAA, New York, 1987, p. 488.

${ }^{43}$ Brown, C.D., Spacecraft Mission Design, AIAA, Reston, VA, 1998, pp.43-45.

${ }^{44}$ Vivona, R.A, Karr, D.A., and Roscoe, D.A., "Pattern-Based Genetic Algorithm for Airborne Conflict Resolution," AIAA Guidance, Navigation, and Control Conference and Exhibit, 21-24 August 2006, Keystone, Colorado, AIAA2006-6060, AIAA, 2006.

${ }^{45}$ Holland, J., Adaptation in Natural and Artificial Systems, University of Michigan Press, Ann Arbor, MI, 1975.

${ }^{46}$ Spencer, H., Principles of Biology, Vol.1, D. Appleton and Company, New York, 1864, p.444.

${ }^{47}$ Lacy, M., "Genetic Algorithms in Java," Java Developer's Journal, Vol.6, No.3, 2001.

${ }^{48}$ De Jong, K.A., "An Analysis of the Behavior of a Class of Genetic Adaptive Systems," PhD Thesis, University of Michigan, Ann Arbor, MI, 1975.

${ }^{56}$ Fonseca, C.M., and Fleming, P.J., "Genetic Algorithms for Multiobjective Optimization: Formulation, Discussion and Generalization," Proceedings of the Fifth International Conference on Genetic Algorithms, edited by S. Forrest, Morgan Kaufmann, San Mateo, CA, 1993, pp.416-423.

${ }^{49}$ Miettinen, K.M., Nonlinear Multiobjective Optimization, Kluwer Academic Publishers, Boston, MA, 1999, pp.11, 78.

${ }^{50}$ Spall, J.C., Introduction to Stochastic Search and Optimization, John Wiley \& Sons, Inc., Hoboken, NJ, 2003, pp.237-242. 
${ }^{51}$ Schaffer, J.D., "Multiple Objective Optimization with Vector Evaluated Genetic Algorithms," Genetic Algorithms and their Applications: Proceedings of the First International Conference on Genetic Algorithms, edited by Lawrence Erlbaum. Lawrence Erlbaum Associates, Inc., Hillsdale, New Jersey, 1985, pp.93-100.

${ }^{52}$ Coello Coello, C.A., Van Veldhuizen, D.A., and Lamont, G.E., Evolutionary Algorithms for Solving Multi-Objective Problems, Kluwer Academic Publishers, Norwell, MA, 2002, p.70.

${ }^{53}$ Holland, J., Adaptation in Natural and Artificial Systems, University of Michigan Press, Ann Arbor, MI, 1975, p.166.

${ }^{54}$ Srinivas, N. and Deb, K., "Multiobjective Optimization Using Nondominated Sorting in Genetic Algorithms," Evolutionary Computations, Vol.2(3), 1994, pp.221-248.

${ }^{55}$ Deb, K., and Goldberg, D.E., "An Investigation of Niche and Species Formation in Genetic Function Optimization," Proceedings of the Third International Conference on Genetic Algorithms, edited by J.D. Schaffer, Morgan Kaufmann Publishers, San Mateo, CA, 1989, pp.42-50.

${ }^{57}$ Goldberg, D.E., and Richardson, J., "Genetic algorithm with sharing for multimodal function optimization," Genetic Algorithms and Their Applications: Proceedings of the Second International Conference on Genetic Algorithms, edited by J.J. Grefenstette, Lawrence Erlbaum Associates, Hillsdale, New Jersey, 1987, pp.41-49.

${ }^{58}$ Union of Concerned Scientists, "UCS Satellite Database," 2006, URL:http://www.ucsusa.org/global_security/space_weapons/satellite_database.h tml [cited 07 Nov.2006].

${ }^{59}$ Freeman, E., Susanne, H., and Arnold, K., JavaSpaces Principles, Patterns, and Practice, Addison-Wesley, Reading, Massachusetts, 1999, pp. 153-155. 
This page is no longer blank 